\title{
Modeling Graphene-Substrate Interactions
}

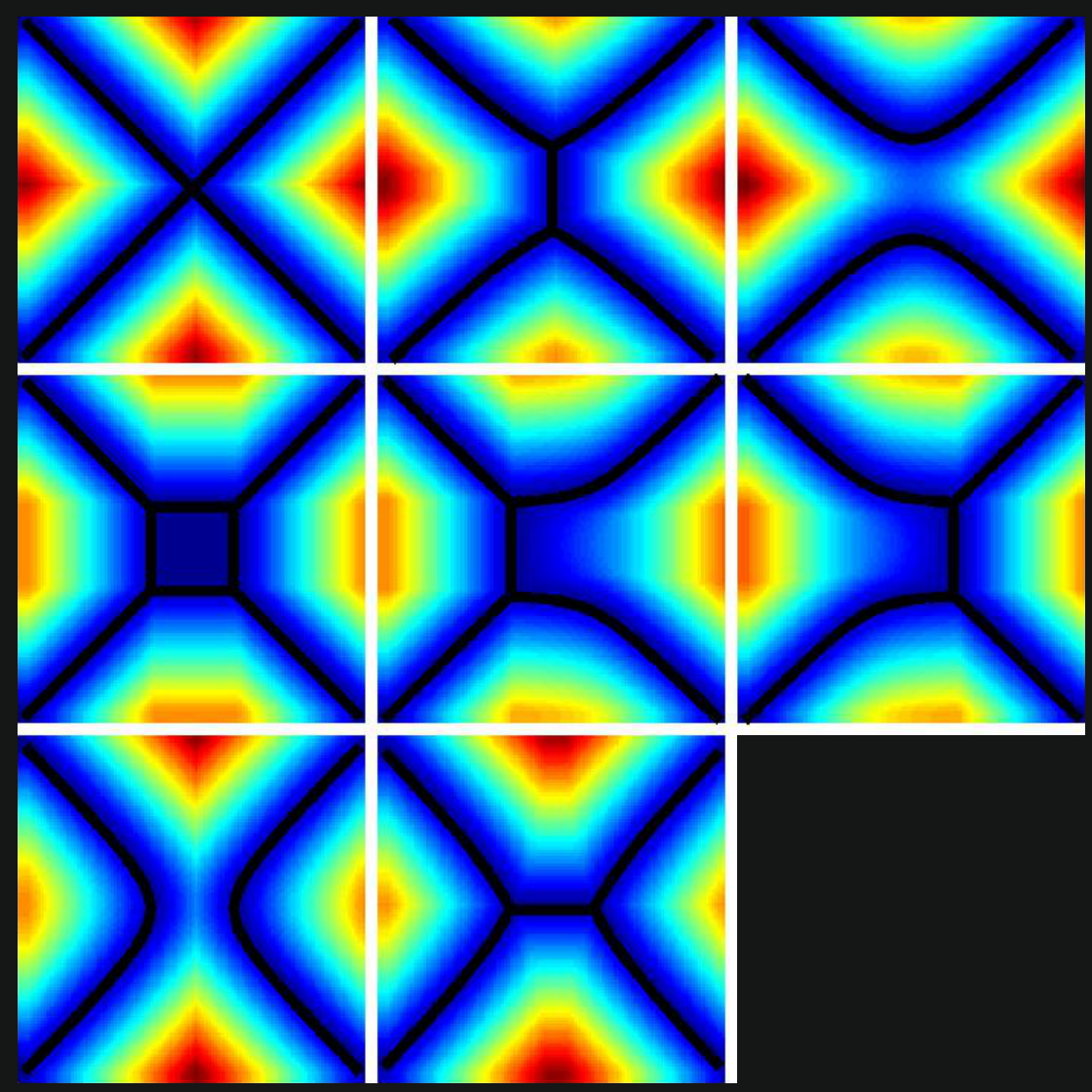




\section{Modeling Graphene-Substrate Interactions}

Taher Amlaki 


\section{Composition of graduation committee:}

Chairman and secretary:

Supervisor:

Members:
Prof. dr. ir. J.W.M. Hilgenkamp (University of Twente)

Prof. dr. P. J. Kelly (Universiteit Twente)

Prof. dr. ir. H.J.W. Zandvliet (Universiteit Twente)

Prof. dr. A.P. Mosk (Universiteit Twente)

Prof. dr. T. Heine (Jacobs University Bremen, Germany)

Prof. dr. M.I. Katsnelson (Radboud Universiteit Nijmegen)

Prof. dr. M.S. Golden (Universiteit van Amsterdam)

The work described in this thesis was carried out in the Computational Materials Science group, MESA+ Institute for Nanotechnology, University of Twente, the Netherlands.

This work is part of the research programme of the Foundation for Fundamental Research on Matter (FOM), which is part of the Netherlands Organisation for Scientific Research (NWO). The use of supercomputer facilities was sponsored by the Physical Science division of NWO (NWO-EW).

Modeling Graphene-Substrate Interactions

$\mathrm{PhD}$ thesis University of Twente, Enschede

ISBN: 978-90-365-4090-2

DOI: $10.3990 / 1.9789036540902$

(C)T. Amlaki, 2016, Enschede, the Netherlands

Published by : T. Amlaki

Printed by: Gildeprint Drukkerijen - Enschede

Cover: "Eight $\lambda_{m}-\lambda_{\text {so }}$ cross-sections of phase space (chapters 4, 5, and 6)".

Author email: taher.amlaki@gmail.com 


\section{MODELING GRAPHENE-SUBSTRATE INTERACTIONS}

\section{DISSERTATION}

to obtain

the degree of doctor at the University of Twente, on the authority of the rector magnificus, prof.dr. H. Brinksma, on account of the decision of the graduation committee, to be publicly defended

on Friday the $8^{\text {th }}$ of April 2016 at 16:45

by

\section{Taher Amlaki}

born on $7^{\text {th }}$ of December 1984

in Ray, Iran 
This dissertation has been approved by: Prof. Dr. P. J. Kelly (promotor) 
To my family 



\section{Contents}

1 Introduction 1

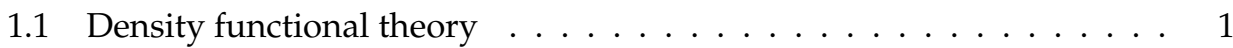

1.1.1 Exchange and correlation functionals ........... 2

1.1.2 Plane wave pseudopotential method ........... 3

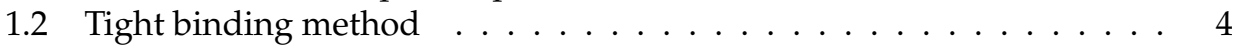

1.3 Novel two-dimensional materials . . . . . . . . . . . . . . . . . 5

1.3.1 Graphene and Germanene . . . . . . . . . . . . . . . . 6

1.3.2 Hexagonal boron nitride . . . . . . . . . . . . . 7

1.3.3 Transition metal dichalcogenides . . . . . . . . . . . . . 7

1.3.4 Litharge $\mathrm{PbO}$ and $\mathrm{SnO} \ldots \ldots \ldots . \ldots 8$

1.4 Topological insulators . . . . . . . . . . . . . . . . . 8

2 Band gaps in incommensurable graphene on hexagonal boron nitride 13

2.1 Introduction . . . . . . . . . . . . . . . 14

2.2 DFT calculations . . . . . . . . . . . . . . . . 15

2.3 Tight binding parametrization . . . . . . . . . . . . . . 16

2.4 Quasiparticle corrections . . . . . . . . . . . . . . . . . . 23

2.5 Solving the Hamiltonian . . . . . . . . . . . . . . . . . 28

2.5.1 Aligned incommensurable graphene $\mid h-\mathrm{BN} \ldots \ldots . \ldots 28$

2.5.2 Misaligned incommensurable graphene $\mid h-\mathrm{BN} \ldots . . . . . .30$

2.6 Löwdin downfolding and effective Hamiltonian . . . . . . . . . . . . . . . . 32

2.7 Perturbation theory for the effective Hamiltonian . . . . . . . . . . 33

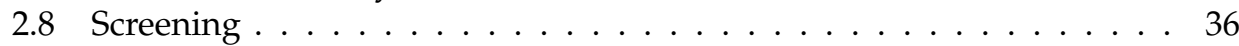

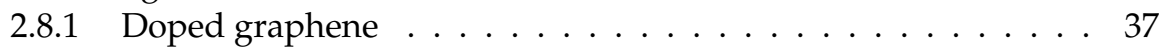

2.8 .2 Undoped graphene . . . . . . . . . . . . . . . 37

2.9 Conclusions . . . . . . . . . . . . . . . . . . . 41

3 Intrinsic e-h puddles in Graphene $\mid \mathrm{hBN} \quad 43$

3.1 Introduction . . . . . . . . . . . . . . . 43

3.2 Computational details . . . . . . . . . . . . . . . . . . . . . . . . . . 44

3.3 Graphene $\mid h$-BN binding . . . . . . . . . . . . . . 45

3.4 Puddle formation . . . . . . . . . . . . . . . . . . . . 46

3.4.1 Low energy Hamiltonian . . . . . . . . . . . . . . . . . 47

3.4 .2 Global solutions . . . . . . . . . . . . . . . . . . 48

3.5 Graphene on molybdenum disulfide . . . . . . . . . . . . . . 50 


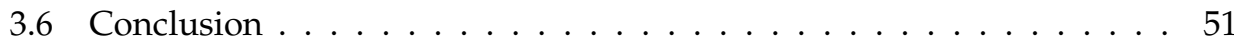

4 Increasing the effective SOC in graphene by hybridization with TMD substrate

4.1 Introduction . . . . . . . . . . . . . . . . 53

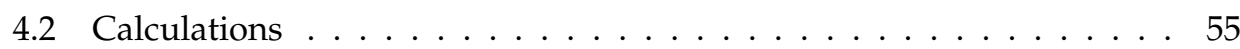

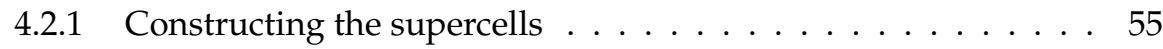

4.2.2 Computational details . . . . . . . . . . . . . . 57

4.2.3 $\mathrm{MX}_{2}$ band structure and band alignments . . . . . . . . . . . . 60

4.3 Constructing the model . . . . . . . . . . . . . . 61

4.3 .1 Asymmetric bilayers ........................... 63

4.3.2 Reflection symmetric trilayers . . . . . . . . . . . . 66

4.3.3 Inversion symmetric trilayers . . . . . . . . . . . . . . 67

4.3 .4 Phase space . . . . . . . . . . . . . . . 67

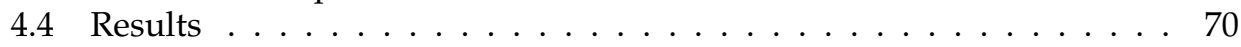

4.4.1 AS parameters and band structure . . . . . . . . . . . . 70

4.4 .2 RS parameters . . . . . . . . . . . . . . . . . . 74

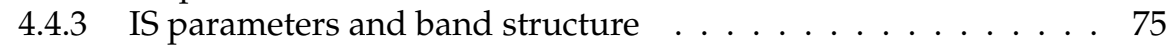

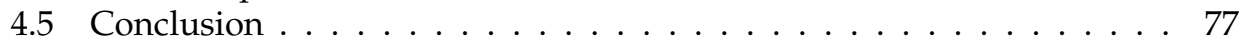

4.6 Appendix: non-zero $\mathbf{f}_{\mathrm{K}} \ldots \ldots \ldots \ldots \ldots$

5 Inducing spin-orbit gap in graphene on heavy metal compounds 81

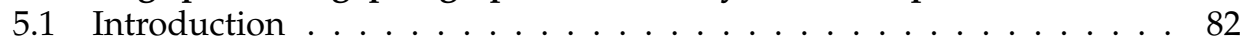

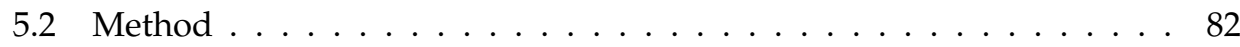

5.3 Band structure and electronic properties of $\mathrm{MX} \ldots \ldots \ldots$. . . . . . 85

5.4 Phenomenological model . . . . . . . . . . . . . . . 87

5.4 .1 Inversion symmetric (IS) supercell . . . . . . . . . . . . . . . . . . 88

5.4 .2 Asymmetrical supercell (AS) . . . . . . . . . . . . . . . . . 89

5.5 Conclusion . . . . . . . . . . . . . . . . 94

$6 \mathbb{Z}_{2}$ invariance of germanene on $\mathrm{MoS}_{2}$ from first-principles 97

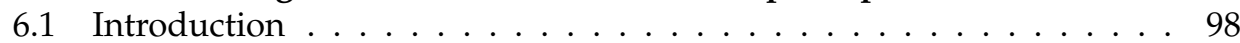

6.2 Phenomenological model: asymmetric bilayer . . . . . . . . . . . . . . 99

6.3 Phenomenological model: inversion symmetric supercell . . . . . . . . 101

6.4 First-principles calculations . . . . . . . . . . . . . . . 101

6.5 Results: AS bilayers . . . . . . . . . . . . . . . . . . . 102

6.6 Results: IS trilayers . . . . . . . . . . . . . . . . . . . . . 104

6.7 Conclusion . . . . . . . . . . . . . . . . . 105

$\begin{array}{ll}\text { Summary } & 107\end{array}$

$\begin{array}{ll}\text { References } & 108\end{array}$

$\begin{array}{ll}\text { Acknowledgement } & 119\end{array}$ 


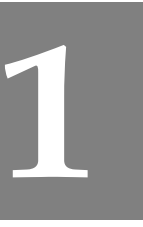

\section{Introduction}

\subsection{Density functional theory}

The electronic structure calculations presented in this thesis are based on the density functional theory (DFT) proposed by Hohenberg and Kohn [1] and Kohn and Sham [2]. DFT has proved to be remarkably successful for calculating structural and electronic properties of materials from first-principles. The theory states that electronic ground state energies and wavefunctions can be calculated from the charge density $-e n(\mathbf{r})$ of a system; the complicated many-body wavefunctions and ground state energy of the system are themselves functionals of the much simpler charge density function. As a result of the variational property of the ground state energy, there is a universal functional $E[n(\mathbf{x})]$ whose minimum determines the electronic ground state energy of the system.

Although the theory assumes the existence of this universal functional, an explicit form is not known so the Hohenberg and Kohn formalism could not be used for calculations as originally formulated. Kohn and Sham then introduced a noninteracting system with the same electron density as the interacting system to obtain a simpler single particle Schrödinger-like equation [2] where the nuclear potentials appear in the form of an "external" potential. The exact expression for the effective potential is not known, but since the Kohn-Sham system is non-interacting then a correspondence between the interacting system and a homogeneous interacting electron gas has led to useful approximations for effective potentials. Foremost among these is the so-called Local Density Approximation (LDA). There, the energy of an inhomogeneous electron gas is approximated locally using the quite well known energy of a homogeneous electron gas with the same density. Improvements based on gradients of the charge density go under the heading "Generalized Gradient Approximations (GGA)". 


\subsubsection{Exchange and correlation functionals}

In addition to the Coulomb potential of the nuclei, the effective potential in the KohnSham scheme comprises the Hartree potential $\left(V_{H}\right)$ of the electron charge density and the exchange-correlation potential $\left(V_{\mathrm{xc}}\right)$. The Hartree term is only the non-interacting classical coulomb potential, and the exchange term $\left(V_{\mathrm{x}}\right)$ is the first order correction that takes into account the anti-symmetry of the electronic wavefunction; the correlation term $\left(V_{\mathrm{c}}\right)$ contains all the other corrections for a many-body electron gas, but it can not be calculated analytically for a general system. For a homogeneous electron gas with density $n$, the exchange-energy density is $\varepsilon_{x}=-\frac{1}{2}\left(3 \pi^{2} n\right)^{1 / 3}$. The energydensity of the homogeneous electron gas is not known exactly as a function of the density but can be approximated using a variety of different many-body techniques ranging from perturbation theory to quantum Monte Carlo simulations. $V_{H}$ and the background ionic positive potentials will cancel for an infinitely large homogeneous system (jellium model) and then the first term in the energy is the kinetic energy which for the noninteracting homogeneous electron gas is known. The expansion parameter in perturbation theory is the electron gas density expressed in terms of $r_{s}$ where $\frac{4 \pi}{3}\left(r_{s} a_{o}\right)^{3} n=1$ (in three-dimensions) where $a_{o}$ is the Bohr radius.

First order perturbation theory yields the exchange energy but higher orders of perturbation theory for the homogeneous electron gas are problematic since each order diverges and therefore cannot be used. However the summation over an infinite number of perturbation orders remains finite. The perturbation terms are so complicated that only some special classes can be analytically summed and only the two cases of the high density and low density (ladder diagrams) limits have been solved. For the high density case, the Random Phase Approximation (RPA) has been used to calculate the correlation potential $\left(V_{\mathrm{c}}\right)$ that has been used for the LDA [3].

Problems that arise because LDA exchange-correlation functionals take inadequate account of the non uniformity of real charge densities can be partly remedied by using functionals with semi-local terms. One class of functional that adds the gradient of the density to the LDA functionals is the GGA $[4,5,6]$. These give better results for the lattice constants and binding energies of many materials but either fail to predict binding for layered materials or predict very small binding energies or too large interlayer separations. This is usually attributed to the interaction between layers having van der Waals character that is not captured by the LDA or the GGA. A non-local term should be added to the local correlation functional to describe this feature. This non-local term is like a Hartree potential but replacing the Coulomb kernel $\left(1 /\left|\mathbf{x}-\mathbf{x}^{\prime}\right|\right)$ with a van der Waals kernel $\left(1 /\left|\mathbf{x}-\mathbf{x}^{\prime}\right|^{6}\right.$ for point particles). Including it yields binding energies and equilibrium separations in better agreement with experiment for layered materials.

In this thesis I have used LDA as the main functional where it gives reasonable binding distances, and GGA and van der Waals wherever necessary. 


\subsubsection{Plane wave pseudopotential method}

In order to make optimal use of Bloch's theorem, we model solids with unit cells that are repeated periodically so that the wavevector $\mathbf{k}$ becomes a good quantum number. The wavefunctions (Bloch waves) for a periodic potential are periodic functions multiplied by a phase that depends on $\mathrm{k}$. The Bloch functions can be expanded using a localized basis with appropriate phases, or delocalized basis. An example of the localized approach is tight binding (TB) which I will discuss in the next section. For the second approach we can choose plane-waves with the periodicity of lattice cells as a basis in which to expand the periodic part of the Bloch function. Since the kinetic energy and the effective potential (of the Kohn-Sham equations) are both periodic in a periodic solid, they can be expanded using a plane-wave basis and the corresponding Schrödinger equation in the solid will have a simple representation. The kinetic energy operator in this basis is diagonal and easily calculated compared to the calculation with a localized basis where calculating matrix elements of the gradient operator is in practice very challenging.

The problem with using a plane wave representation is that the Coulomb potential close to the nuclei diverges. To accurately reproduce the rapid oscillations of atomic orbitals in this region, a very large number of plane waves would be needed to describe the part of the Hamiltonian and wavefunctions that vary rapidly close to the nuclei. This problem is solved in the "pseudopotential" method by replacing the diverging and problematic real potential inside a "core" sphere close to the nuclei with a "pseudo"potential that is much weaker but has the same scattering properties; outside this sphere the pseudopotential is identical to the atomic potential. The solutions of the Schröodinger equation for a pseudopotential are called pseudo wavefunctions.

With "norm-conserving" pseudopotentials [7] the core radius and pseudopotential have to be chosen in such a way that the eigenenergies are the same as the eigenenergies with the real potential, while the pseudo-wave functions match the real wavefunctions at (and beyond) the boundary of the core sphere. Requiring the norm of the pseudo-wave function to be the same as the real wavefunction ensures that the charge is the same for both pseudo and real wavefunctions, inside and outside of the sphere and the energy dependence of the scattering is correct to first order.

In the Projector Augmented Wave (PAW) method [8] which is used in the Vienna $\mathrm{Ab}$-initio Simulation Package (VASP), the pseudo wavefunction $\tilde{\psi}_{i}$ is identical to the real wavefunction $\psi_{i}$ outside the core sphere. Inside the core sphere the pseudo wavefunction is defined in terms of a slowly varying pseudo partial wave $\tilde{\phi}_{i}$. The two are related by subtracting the pseudo partial wave $\tilde{\phi}_{i}$ and adding the correct partial wave $\phi_{i}$ as

$$
\left|\psi_{n}\right\rangle=\left|\tilde{\psi}_{n}\right\rangle+\sum_{i}\left\{\left|\phi_{i}\right\rangle-\left|\tilde{\phi}_{i}\right\rangle\right\}\left\langle\tilde{p}_{i} \mid \psi_{n}\right\rangle
$$

where the projector functions $\left\langle\tilde{p}_{i}\right|$ are dual to the pseudo partial waves; $\left\langle\tilde{p}_{i} \mid \tilde{\phi}_{j}\right\rangle=$ $\delta_{i j}$. Since the pseudo partial waves $\left|\tilde{\phi}_{i}\right\rangle$ are not orthonormal and partial waves at 
different atomic positions are not orthogonal then dual waves $\left|\tilde{p}_{i}\right\rangle$ are not atomic like. This method was developed by Blöchl [8] and is implemented in the VASP [9] code that I use in this thesis.

The wavefunction can be decomposed into components inside and outside the core spheres

$$
\left|\psi_{n}\right\rangle=\left\{\begin{array}{l}
O \rightarrow\left|\tilde{\psi}_{n}\right\rangle \\
I \rightarrow \sum_{i}\left|\phi_{i}\right\rangle\left\langle\tilde{p}_{i} \mid \tilde{\psi}_{n}\right\rangle
\end{array}\right.
$$

where $O$ represents the outside regions and $I$ represents the inside regions. We have used the fact that in the outside region $\left|\phi_{i}\right\rangle=\left|\tilde{\phi}_{i}\right\rangle$ and in the inside region $\sum_{i}\left|\tilde{\phi}_{i}\right\rangle\left\langle\tilde{p}_{i}\right|=1$ so we have

$$
\left\langle\psi_{n} \mid \psi_{m}\right\rangle=\left\langle\tilde{\psi}_{n}|W| \tilde{\psi}_{m}\right\rangle
$$

where $W$ is the overlap operator

$$
W=1+\sum_{i}\left|\tilde{p}_{i}\right\rangle\left\{\left\langle\phi_{i} \mid \phi_{j}\right\rangle-\left\langle\tilde{\phi}_{i} \mid \tilde{\phi}_{j}\right\rangle\right\}\left\langle\tilde{p}_{j}\right| .
$$

When spin-orbit interactions are included then we can extend this formulation to calculate the spin characters of the bands. For example for $s_{z}$ we have

$$
\left\langle\psi_{n}\left|s_{z}\right| \psi_{m}\right\rangle=\left\langle\tilde{\psi}_{n}^{\uparrow}\left|W^{\uparrow}\right| \tilde{\psi}_{m}^{\uparrow}\right\rangle-\left\langle\tilde{\psi}_{n}^{\downarrow}\left|W^{\downarrow}\right| \tilde{\psi}_{m}^{\downarrow}\right\rangle
$$

therefore the spin matrices can be calculated if we orthogonalize the $\tilde{\psi}_{n}$. The matrix that orthogonalizes the $\tilde{\psi}_{n}$ waves can be found by then diagonalizing the overlap matrix and scaling the pseudo-waves to obtain the spin matrix, $|\varphi\rangle=V|\tilde{\psi}\rangle$ where, $V$ is a matrix, and the $\varphi$ are an orthogonal basis, so we have

$$
\left\langle\psi_{n}|\mathbf{s}| \psi_{n}\right\rangle=\left\langle\varphi_{n}|\mathbf{s}| \varphi_{n}\right\rangle
$$

We will use this relation to calculate spin matrices in chapters 4,5 , and 6 .

\subsection{Tight binding method}

The tight binding (TB) model is another approach to calculate the electronic band structure of materials. It uses a (minimal) local basis to expand the wavefunctions and takes the matrix elements of the Hamiltonian as functions of wavevector, the phases of the elements being determined by the geometry of the lattice. Since the TB model cannot easily be used to determine the parameters of the Hamiltonian directly, we need to develop other ways to determine them from explicit first-principles calculations. However, the lattice symmetries can be incorporated into the Hamiltonian without the need to parameterize and therefore it is useful to gain an understanding of band symmetries.

The TB picture is used in two ways in this thesis. First, since for very large sys- 
tems we cannot perform direct first-principles calculations, we parametrize a TB Hamiltonian for local configurations and then apply it to larger systems. Since TB only requires determining the dependence of matrix elements of the Hamiltonian on the local environment, then it is much faster than DFT and can be applied to large systems. The second use that we make of the TB model is to understand the spin-orbit interactions of graphene on a substrate, to construct and interpret an effective low-energy independent particle Hamiltonian for graphene on a substrate (graphene|substrate) and in principle any four band system which resembles graphene.

Parametrization of the tight binding model in this thesis is based upon the SlaterKoster formulation [10] of a two-center approximation for the Hamiltonian matrix elements

$$
H_{n m}(\mathbf{R})=\int \psi_{n}^{*}(\mathbf{x}) H \psi_{m}(\mathbf{x}-\mathbf{R}) d^{3} \mathbf{x}
$$

where $\psi_{n}(\mathbf{x})$ is an atom-centred basis and the spatial dependence of the matrix element only comes from $\mathbf{R}$ (this statement is not strictly true but the two-center approximation works well for many situations). By using a localized atomic-like basis, we can identify the contributions of different atoms and orbitals to the electronic structure. For example, we can parameterize a tight binding Hamiltonian with $s p^{3}$ orbitals for buckled germanene. Then the tight binding immediately shows how the $s p^{2}$ orbitals mix with the $p_{z}$ orbitals for low-energy bands. If we calculate the spin-orbit coupling coefficent from first-principles calculations for a single atom of germanium then we can add spin-orbit interactions to the tight binding Hamiltonian and predict the band structure of germanene with spin-orbit interactions. Because it has inversion symmetry, germanene will not have any band gap without spin-orbit coupling, but with them it will. Tight binding can predict this gap and also we can recognize the contribution of different orbitals to this gap. These abilities of tight binding, to predict the general shape of the band structure with relatively low computational cost and to yield "chemical" information about the nature of the bands make it a very popular method for explaining the band structures of materials.

\subsection{Novel two-dimensional materials}

Starting with graphene, a single layer of carbon atoms extracted from graphite, huge experimental advances have been made in the past 10 years in preparing isolated layers of two-dimensional materials. This has opened up exciting new fields in condensed matter physics since the electrons are free only in two dimensions and confined in the third dimension. This confinement also means that the two-dimensional layers are weakly bonded when stacked in bulk structures and therefore can be extracted and transferred so they can be combined in many different ways. Weak binding formally translates into bonding by van der Waals interactions. The idea of using layers like Lego blocks was suggested relatively recently and is a very active field of study [11]. In this thesis I study the interactions of graphene and its proposed 

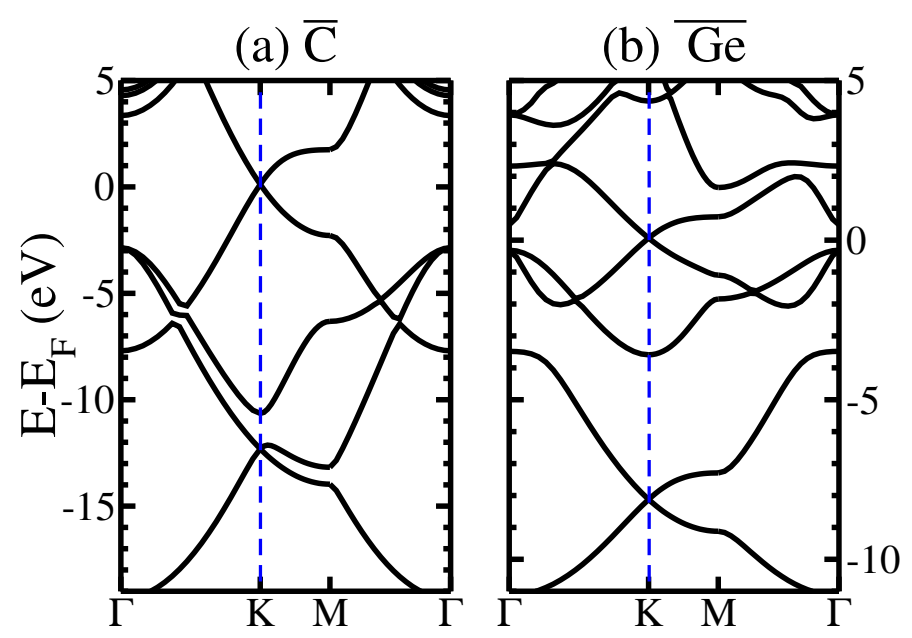

Figure 1.1: Band structure of (a) graphene $(\overline{\mathrm{C}})$ and $(\mathrm{b})$ buckled germanene $(\overline{\mathrm{Ge}})$. The Dirac cone is around the Fermi energy $\left(E_{\mathrm{F}}=0 \mathrm{eV}\right)$ at the $\mathbf{K}$ point.

analogue based upon germanium, germanene, with a number of two-dimensional substrates.

\subsubsection{Graphene and Germanene}

Graphene and Germanene are layers of atoms arranged in hexagons only a single atom thick and consisting of a single type of atom, carbon and germanium. The symmetry of this "honeycomb" lattice leads to the existence of a "Dirac cone" in the band structure at the Fermi energy arising from the crossing of two (spin degenerate) bands with linear dispersion at the high-symmetry $\mathbf{K}$ point in the absence of spinorbit interactions $[12,13,14]$. The band structures of graphene and germanene are shown in Fig. 1.1. The bands are linear up to about $1 \mathrm{eV}$ for graphene and $0.5 \mathrm{eV}$ for germanene. An important property of graphene is its large room-temperature carrier mobility, ten times higher than that of silicon [15] which promises to be useful in making transistors with graphene. Since the charge carrier mobility of graphene is higher, higher currents can be applied to the transistor with higher frequency and lower energy consumption - in principle. The drawback is the gapless band structure of graphene since a band gap is needed for logic applications. This makes a study of gap opening mechanisms in graphene of interest. Using a substrate to break sublattice symmetry in graphene is the topic of study in chapter 2. Spin-orbit interaction can also open a band gap in graphene and this is studied in chapters 4 and 5 . The intrinsic spin-orbit interaction is minuscule in graphene. Even when a substrate is used to induce a larger spin orbit interaction, the greatly increased gap is still tiny. The atomic splitting due to spin-orbit coupling increases rapidly with the atomic number $Z$ so heavier elements like silicon $(Z=14)$ and germanium $(Z=32)$ have 

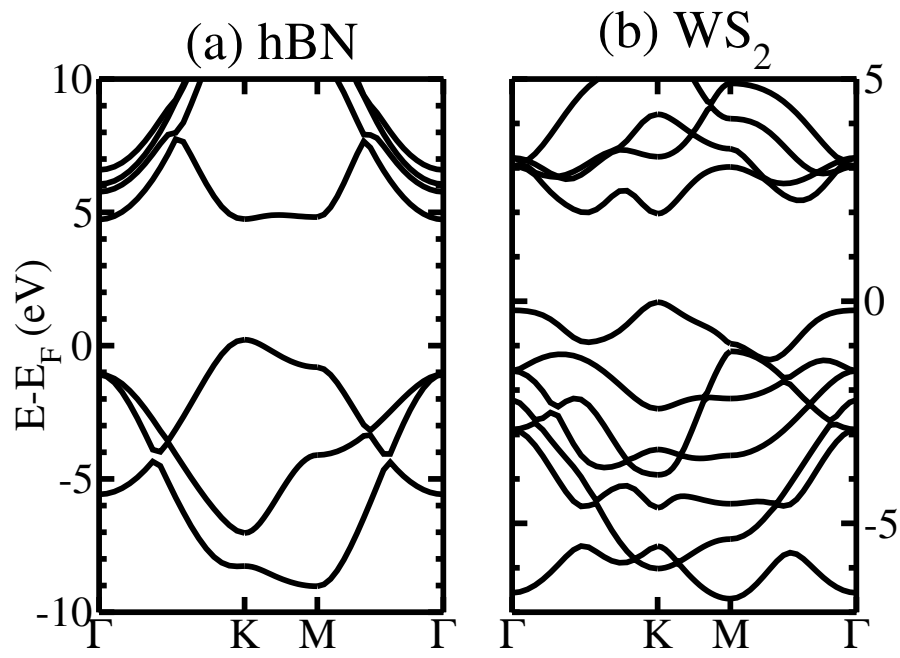

Figure 1.2: Band structure of (a) monolayer of hexagonal boron nitride (hBN) and (b) trilayer of $\mathrm{WS}_{2}$.

much larger spin-orbit coupling (and SOC induced band gaps) than carbon $(Z=6)$. We study the effect of a substrate on the band structure and gap of germanene in chapter 6 .

In this "tetragene" family two other materials can be found, silicene and stanene. Silicene has spin-orbit coupling between graphene and germanene, and stanene has the largest SOC in this family. However the stable monolayer structure of graphene, silicene, and germanene are hexagonal lattice, while stanene has a different lattice structure $[14,16,17]$.

\subsubsection{Hexagonal boron nitride}

Hexagonal boron nitride ( $h$-BN) has the same lattice structure as graphene but contains two different atoms, boron and nitrogen [18]. This property makes $h$-BN an insulator and $h$-BN doesn't have a Dirac cone. The band gap is about $6 \mathrm{eV}$, and therefore $h$-BN can be a good substrate. Its large band gap makes $h$-BN inert in comparison with graphene and germanene and therefore using $h-\mathrm{BN}$ as a substrate will not change their band structures drastically. The band structure of $h$-BN is shown in Fig. 1.2(a).

\subsubsection{Transition metal dichalcogenides}

Like $h$-BN, the $\mathrm{MX}_{2}$ transition metal dichalcogenides (TMD) are insulators but their band gaps are much smaller by comparison, only 1-2 eV [19]. Viewed from above, the lattice structure of $\mathrm{MX}_{2}$ is hexagonal similar to $h$ - $\mathrm{BN}$, but from the side the struc- 
ture looks quite different, with the $\mathrm{X}$ atoms arranged in planes above and below the transition metal plane; if the $\mathrm{M}$ atoms are on the boron sublattice, the two $\mathrm{X}$ atoms form a dumbbell normal to the $\mathrm{M}$ plane on the nitrogen. Like graphene and $h$-BN, a monolayer of $\mathrm{MX}_{2}$ can be cleaved from a bulk crystal because the (tri)layers are weakly bound. The mobility of the charge carriers in $\mathrm{MX}_{2}$ is lower in comparison with graphene, but still high enough to make them useful for application in electronic devices [20, 21, 22]. The band structure of $\mathrm{WS}_{2}$ is shown in Fig. 1.2(b).

\subsubsection{Litharge $\mathrm{PbO}$ and $\mathrm{SnO}$}

We explore using $\mathrm{PbO}$ and $\mathrm{SnO}$ as substrates for graphene because these materials contain heavy atoms $\mathrm{Pb}$ and $\mathrm{Sn}$ with large spin-orbit coupling that might be transfered to graphene by hybridization. The litharge structure of $\mathrm{PbO}(\alpha-\mathrm{PbO})$ and $\mathrm{SnO}$ ( $\alpha$-SnO) is like the $\mathrm{CsCl}$ structure [23, 24]. The $\alpha$ - $\mathrm{PbO}$ and $\alpha$-SnO have two-fold rotational symmetry around the central oxygen atom, and therefore putting graphene on them will break its $C_{3}$ symmetry. The resulting supercell cannot have rotational symmetry and this is interesting from a theoretical point of view.

\subsection{Topological insulators}

Topological insulators are materials with bulk band gaps, but with robust conducting edge states. The robustness of these edge states is guaranteed by the topological nature of their bulk bands [25] that is determined by global properties of the Hilbert space spanned by their wavefunctions [26]. In crystals where the wavevector $\mathbf{k}$ is a good quantum number, then the map from $\mathbf{k}$ space into the Hilbert space determines how the topological invariant can be calculated. An important local quantity is the Berry curvature $B_{n}(\mathbf{k})$ which is related to the derivatives of the wavefunction with respect to the wavevector. It can be expressed as [27]

$$
B_{n}(\mathbf{k})=i \nabla_{\mathbf{k}} \times\left\langle u_{n}\left|\nabla_{\mathbf{k}}\right| u_{n}\right\rangle
$$

where $\left|u_{n}\right\rangle$ is the Bloch cell-periodic function. The Berry curvature is independent of the choice of gauge for the wavefunctions and can therefore be considered as a physically observable quantity. It can be related to matrix elements of the $k$-space gradient of the Hamiltonian by

$$
B_{n}=\mathfrak{I} \sum_{m \neq n} \frac{\left\langle u_{n}\left|\nabla_{k} H\right| u_{m}\right\rangle \times\left\langle u_{m}\left|\nabla_{k} H\right| u_{n}\right\rangle}{\left(\varepsilon_{m}-\varepsilon_{n}\right)^{2}}
$$

where $\varepsilon_{n}$ is the energy of the $n$th band. The global property that is the topological invariant can be defined as the integral of the Berry curvature over the Brillouin zone.

$$
C_{n}=\frac{1}{2 \pi} \int d^{2} \mathbf{k} B_{n}(\mathbf{k}) .
$$




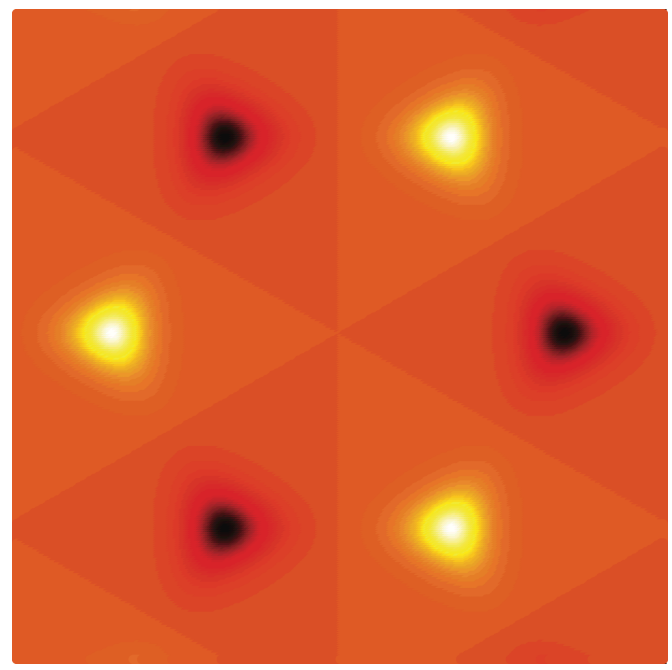

Figure 1.3: Berry curvature of graphene calculated from nearest neighbour tight binding $p_{z}$ orbital bands. The light and dark colors illustrate positive and negative Berry curvature. For the sake of illustration we have added a finite mass term to open a gap, otherwise for pristine graphene the Berry curvature would be infinite at $\mathbf{K}$ (light coloured) and $\mathbf{K}^{\prime}$ (dark coloured).

$C_{n}$, the Chern number of the $n$th band is a topological invariant meaning that it does not change under small perturbations. Because it is an integer changing it requires a relatively large perturbation. Band crossing can lead to infinite Berry curvature at the crossing point (because the denominator in Eq. 1.9 becomes zero) and therefore the Chern number of a particular band might change at the crossing point, moreover the band number also changes so the Chern number calculated by following the band by its band number can be problematic. However, the sum of Berry curvatures of two crossing bands remains finite (because the numerator in Eq. 1.9 is odd under exchange of $n$ and $m$ ), and is therefore meaningful and independent of accidental crossings of the bands. Since an insulator has a finite gap, the Chern number of occupied bands can be defined. The electrons are in the occupied bands so the Chern number of occupied bands of an insulator is the topological invariant of that material.

Topological insulators are categorized by their topological invariants. The first 
class of topological insulators are called quantum Hall insulators. They occur when electrons are confined at the boundary by a strong magnetic field, and as a result the Hall conductivity will be quantized [28].

The second class of topological insulators are called $\mathbb{Z}_{2}$ topological insulators $[29,30]$ and they represent spin quantum Hall states, also with robust edge states at their boundaries. The spin and momentum of electrons in the edge states are coupled, the total charge current will be zero but the spin current is non-zero [31]. This locking of spin and momentum originates from non-trivial spin-orbit interactions in the system, so spin-orbit coupling is important in a material for the $\mathbb{Z}_{2}$ invariant to be non-trivial [32].

The $\mathbb{Z}_{2}$ topological invariant reflects the interplay of interactions and symmetry in the system. As an example, I show the Berry curvature for a graphene-like system of an hexagonal lattice with three-fold rotational symmetry in Fig. 1.3. We used a simple nearest neighbour tight binding approximation to obtain the eigenvalues and wavefunctions for this system and plotted the Berry curvature of occupied bands throughout the Brillouin zone. As shown in the figure, the Berry curvature diverges at the high symmetry points $\left(\mathbf{K}\right.$ and $\left.\mathbf{K}^{\prime}\right)$ at the boundary of the Brillouin zone. It has opposite signs at $\mathbf{K}$ (light regions) and $\mathbf{K}^{\prime}$ (dark regions) and the integral of the occupied band Berry curvature over the Brillouin zone will be zero. This is because the system has time-reversal symmetry, but there is information in the half-Brillouin zone that determines the $\mathbb{Z}_{2}$ topological invariant. We have calculated the Berry curvature for a two-dimensional square lattice with the same hopping parameters but the Berry curvature remains zero everywhere even at the Brillouin zone boundaries. This shows that the lattice symmetries of the material play an important role in determining the global properties of the Hilbert space of the system. A more comprehensive study of the relationship between lattice symmetries and topological invariants has been given by Slager et al [33].

For graphene-like systems the $\mathbb{Z}_{2}$ topological invariant is related to the integral of the Berry curvature over the half-Brillouin zone, which in the low-energy models can be related to the effective Hamiltonian around the $\mathbf{K}$ or $\mathbf{K}^{\prime}$ points. The $\mathbb{Z}_{2}$ invariant for such systems is formalized in chapters 4,5 , and 6 . Althought we can perform first-principles calculations for graphene on a substrate by approximating the structure using supercells, the calculations are much too expensive to directly calculate the topological invariant from the Berry curvature. Instead, we can use the effective four band Hamiltonian

$$
H_{\mathbf{K}}^{\mathrm{AS}}(\mathbf{q})=\hbar v_{F} \mathbf{q} \cdot \boldsymbol{\sigma}+\lambda_{m} \sigma_{z}+\frac{\lambda_{R}}{2}(\boldsymbol{\sigma} \times \mathbf{s})_{z}+\lambda_{\mathrm{so}} \sigma_{z} s_{z}+\lambda_{B} s_{z}
$$

that is derived in Chapter 4 and describes the low energy electronic structure very well to determine the Berry curvature and topological invariant. In addition to the Fermi velocity, the Hamiltonian contains four parameters: a "mass" term $\lambda_{m}$; Kane and Mele's "intrinsic" spin-orbit term $\lambda_{\text {so }}$; the Rashba term $\lambda_{R}$; and a new "pseudomagnetic" term $\lambda_{B}$. We gain insight into what determines the invariant by calculat- 


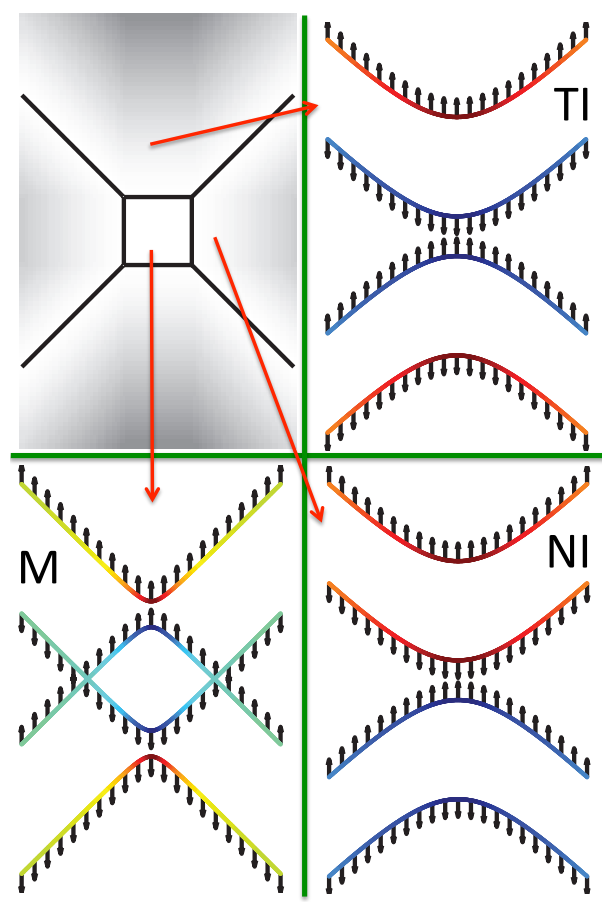

Figure 1.4: Phase space (top-left) and typical band structures corresponding to the different phases. In this figure we have set the Rashba parameter $\lambda_{R}=0$. The boundaries between different phases are shown as black lines while the gray scale represents the magnitude of the band gap, white being zero band gap. The arrows indicate the spin-expectation values of each band, the color indicates the projection onto sublattices ( $\mathrm{A}$ is red, $\mathrm{B}$ is blue).

ing the phase space of the effective Hamiltonian determined by the four parameters $\lambda_{m}, \lambda_{\mathrm{so}}, \lambda_{R}$, and $\lambda_{B}$.

Because the parameter space is four dimensional, only representative cross sections are shown in Fig. 1.4 for graphene on a transition metal dichalcogenide. The top left panel of the figure shows a $\lambda_{m}-\lambda_{\text {so }}$ cross section with $\lambda_{R}=0$ and $\lambda_{B} \neq 0$. For a time-reversal system there can be three distinct phases: topological insulating (TI), normal insulating (NI), and metallic (M). The black lines are boundaries between these phases where the band gap vanishes. In the other panels illustrative band structures corresponding to each region in the parameter space are shown where the color of each band is a measure of the sublattice contribution. The spin expectation value of each band is indicated as a variable length arrow. Different phases are seen to be characterized by different spin-sublattice character of the occupied and unoccupied bands. 

graphene on hexagonal boron nitride

To describe the electronic structure of incommensurable graphene on a hexagonal boron nitride substrate, we develop a minimal tight binding (TB) model whose parameters are fit to the results of first-principles density functional theory and $G W$ many-body perturbation theory calculations for commensurable systems. We approximate incommensurable systems with large supercells and use the TB model to explain recent experiments. The gap observed in aligned incommensurable structures is attributed to incomplete cancellation of the sublattice symmetry-breaking potential and a combination of many-body gap enhancement and modulation of the height of the graphene above the substrate. The gap is very sensitive to orientational misalignment of the graphene layer with the boron nitride substrate. The effect of screening for the misaligned case is also studied. 


\subsection{Introduction}

The aesthetic appeal of near-perfect two-dimensional lattices only a single or a few atoms thick [34] combined with intriguing physical properties [15, 35, 36] and promising application potential [37] accounts for the huge attention such materials have received in recent years $[38,11]$. To make useful devices, graphene $(\overline{\mathrm{C}})$ needs to be deposited on an electrically insulating substrate such as $\mathrm{SiO}_{2}$ or $\mathrm{SiC}$ [39]. However, the interaction with the substrate limits the carrier mobility [40] while the absence of a band gap makes it difficult to realize a non-conducting, "off" device state. This has led to suggestions for alternative substrates with more suitable properties [18, 41]. For example, hexagonal boron nitride $(h-\mathrm{BN})$ is a layered material with the same honeycomb structure as graphene but is a large band gap insulator. Calculations that neglected the small $1.8 \%$ lattice mismatch indicated that the weak interaction with graphene might open a small band gap of about $50 \mathrm{meV}$ [18]. Dean and coworkers succeeded in developing a procedure to prepare graphene on a $h$-BN substrate and found a much improved mobility [42]. Scanning tunneling microscopy (STM) studies $[43,44]$ shortly afterwards failed to observe the predicted bandgap opening. Xue et al. suggested that the reason might be related to the moire patterns they observed in their STM studies that were compatible with the expected incommensurablity of graphene and $h$-BN. Using first-principles total energy calculations with an improved description of exchange and correlation to estimate deformation and binding energies, Sachs et al. argued that the binding energy gained by graphene and $h$-BN becoming commensurable was comparable to the energy cost of stretching graphene or compressing $h$-BN making the assumption of commensurability too strong [45]. Tight-binding (TB) analyses led Xue [43] and Sachs [45] to argue that the symmetry-breaking interaction between graphene and $h$-BN would lead to a gap with a maximum value of $\sim 5 \mathrm{meV}$ [45], much smaller than the $\sim 50 \mathrm{meV}$ predicted for commensurable structures $[18,45]$.

The situation appeared to be resolved in spite of anomalous low temperature transport behaviour [46, 47] until clear Arrhenius like behaviour was observed in the conductivity with sample-dependent gaps as large as $27 \mathrm{meV}$ [48]. In particular, a correlation between the size of the gap and the relative orientation of graphene and $h$-BN was observed. Because the absence or smallness of the gap poses a limitation to the application of graphene in transistors, for which larger band gaps of at least 400 $\mathrm{meV}$ are required $[49,37,39]$, it is important to understand the band gap opening mechanism in single-layer graphene deposited on a substrate to be able to make constructive use of the possibilities offered by graphene-substrate interactions.

Incommensurability of graphene and $h$ - $\mathrm{BN}$, as evidenced by moiré patterns observed with STM, plays a key role in quenching $[43,45,50]$ the sublattice symmetry breaking interaction that led to the originally proposed gap opening [18]. However, this is only one element that should be taken into account in modelling $\overline{\mathrm{C}} \mid h$-BN more realistically. Other factors that may play an important role are (i) many-body effects [51, 52], (ii) deviation of the graphene from an ideal planar form parallel to a planar 
$h$-BN, (iii) local interface potential steps, and (iv) the degree of incommensurability; the moiré pattern considered by Sachs et al. was about $4 \mathrm{~nm}$ in length, substantially smaller than the patterns of order $14.5 \mathrm{~nm}$ observed in experiment [43, 44].

To take into consideration the electron hoppings, the sublattice symmetry-breaking potential, and the local interface potential step in this paper, we will set up a minimal tight-binding model for a general commensurable configuration of the $\overline{\mathrm{C}} \mid h-\mathrm{BN}$ system. By applying this local TB model to commensurable configurations that can be studied at the level of density functional theory (DFT) and many-body perturbation theory (MBPT), we will determine the dependence of the hopping matrix elements and potentials on the local geometries. The TB parameters will be found by assuming that the global properties of the system can be extrapolated from the corresponding local properties. The assumption of locality then allows us to extend the TB model to determine the band gaps for a number of approximants of incommensurable configurations. For a misaligned $\overline{\mathrm{C}} \mid h$-BN system, these gaps will be found to be negligible. For an aligned incommensurable parallel planar $\overline{\mathrm{C}} \mid h$-BN at the level of DFT, we will find a gap of $\sim 4 \mathrm{meV}$ in agreement with previous findings [43, 45]. We extend the above TB model to include quasiparticle corrections at the level of $G W$ MBPT and local geometry dependent height variation to find a band gap of about $30(\mathrm{meV})$ for the aligned system in good agreement with the latest experimentally reported gaps [48].

The results of perturbation theory are found to compare well with those obtained from the above TB model. This perturbation scheme helped us understand that the effective many-body corrected sublattice symmetry-breaking potential and height modulation are the most significant factors influencing the band gap. Finally, screening of the potentials have shown that their magnitude drops with about 40 percent for misaligned large supercells. We have not considered the effect of in-plane stresses on the gap opening. There have been attempts to explain the gap opened in $\overline{\mathrm{C}} \mid h$ BN system in terms of in-plane stresses $[53,54]$ where the graphene layer adjusts structurally to the $h$-BN layer for large moiré periodicities at the boundaries of the supercell $[55,56]$. A short report of this work was given in Ref. (Bokdam:prb14a).

\subsection{DFT calculations}

We begin with the three high symmetry aligned commensurable configurations of graphene on $h$-BN identified by Giovannetti et al. and studied in the local density approximation (LDA) [18] and in the random phase approximation (RPA) in the framework of the "adiabatic connection fluctuation dissipation theorem" (ACFDT) [45]. These are the (a) configuration with one carbon over B, the other over $\mathrm{N}$, the (b) configuration with one carbon over $\mathrm{N}$, the other centered above a $h$-BN hexagon, and the (c) configuration with one carbon over $\mathrm{B}$, the other centered above a $h$-BN hexagon sketched in Fig. 2.1. The "RPA" equilibrium separations of $d_{\mathrm{eq}}^{\mathrm{RPA}}=3.55$, 3.50 and $3.35 \AA$ for the (a), (b) and (c) configurations reported by Sachs et al. are the most reliable currently available and we will use these throughout (rather than the 
slightly lower values of $d_{\mathrm{eq}}^{\mathrm{LDA}}=3.50,3.40$ and $3.22 \AA$ predicted by LDA calculations [18]). For a given atomic configuration, electronic structures do not depend critically on the functional used. In particular, Bokdam et al. demonstrated that the interface dipole does not depend on the functional used for a given geometry [57]. For this reason we will use the LDA [3] and the plane-wave projector augmented wave (PAW) method [8], as implemented in VASP [58, 59, 9], to determine the electronic structure of a graphene sheet on top of a $h$-BN substrate as a function of the height $d$ and lateral displacement $(x, y)$ of the two parallel lattices. A plane-wave basis with a cutoff energy of $400 \mathrm{eV}$ is used in combination with a $36 \times 36 k$-point grid (in a $1 \times 1$ unit cell).

Figures 2.1(a)-2.1(c) show the LDA energy bands for graphene on $h$-BN for the (a), (b) and (c) configurations at their RPA equilibrium separations [45] with LDA gaps $\Delta \varepsilon \equiv \varepsilon_{3}(K)-\varepsilon_{2}(K)$ of $30-40 \mathrm{meV}$ opened at the $K$ point by the symmetrybreaking interaction with the substrate [18]. The Dirac point is situated asymmetrically in the $h$-BN band gap about a third of the way from the top of the valence band. Apart from the formation of the small gap, the dispersion of the $\pi$ bands is largely unchanged by the interaction with the $h-\mathrm{BN}$ substrate within about $1 \mathrm{eV}$ of the Dirac point.

If we expand the energy scale about the Dirac point, we see that the centers of the gaps, $\left[\varepsilon_{2}(\mathrm{~K})+\varepsilon_{3}(\mathrm{~K})\right] / 2$, do not coincide for the different configurations (lower panels); the interaction between graphene and $h$-BN gives rise to an interface potential step $\Delta V$. The source of this potential step is a configuration-dependent interface dipole layer that can be visualized in terms of the electronic displacement $\Delta n(\mathbf{r})$ obtained by subtracting the electron densities of the isolated constituent materials, $n_{\mathrm{Gr}}$ and $n_{\mathrm{BN}}$, from that of the entire system $n_{\mathrm{Gr} \mid \mathrm{BN}}[60,61,57]$. The potential step is related to the dipole layer, illustrated in Fig. 2.1 for the (a), (b) and (c) configurations, by $\Delta V=-e^{2} /\left(A \epsilon_{0}\right) \int z \Delta n(\mathbf{r}) d x d y d z$ where $A$ is the area of the surface unit cell and the integration is over all space.

The potential step depends sensitively on how the graphene and $h$-BN lattices are positioned. Starting from the lowest energy (c) configuration, and displacing graphene laterally by $(x, y)$ yields the potential landscape $\Delta V(x, y)$ shown in Fig. 2.2(a). For each value of $(x, y)$, the graphene sheet is a distance $d_{\mathrm{eq}}(x, y)$, the RPA equilibrium separation, from the $h$-BN substrate. $\Delta V$ reaches appreciable values ranging from -20 to $90 \mathrm{meV}$, where the minimum and maximum values are found for the high symmetry (b) and (c) configurations, respectively. The full $d$-dependence of $\Delta V$ is shown for the three configurations in Fig. 2.2(b).

\subsection{Tight binding parametrization}

In this section we develop the tight binding model to describe the low-energy electronic structure of commensurable $\overline{\mathrm{C}} \mid h$-BN systems that was used to fit the first principles LDA band structures in Fig. 2.3 that will then be extended to describe incommensurable systems. We first decompose the TB-Hamiltonian $H_{\overline{\mathrm{C} \mid \mathrm{BN}}}$ of the compos- 


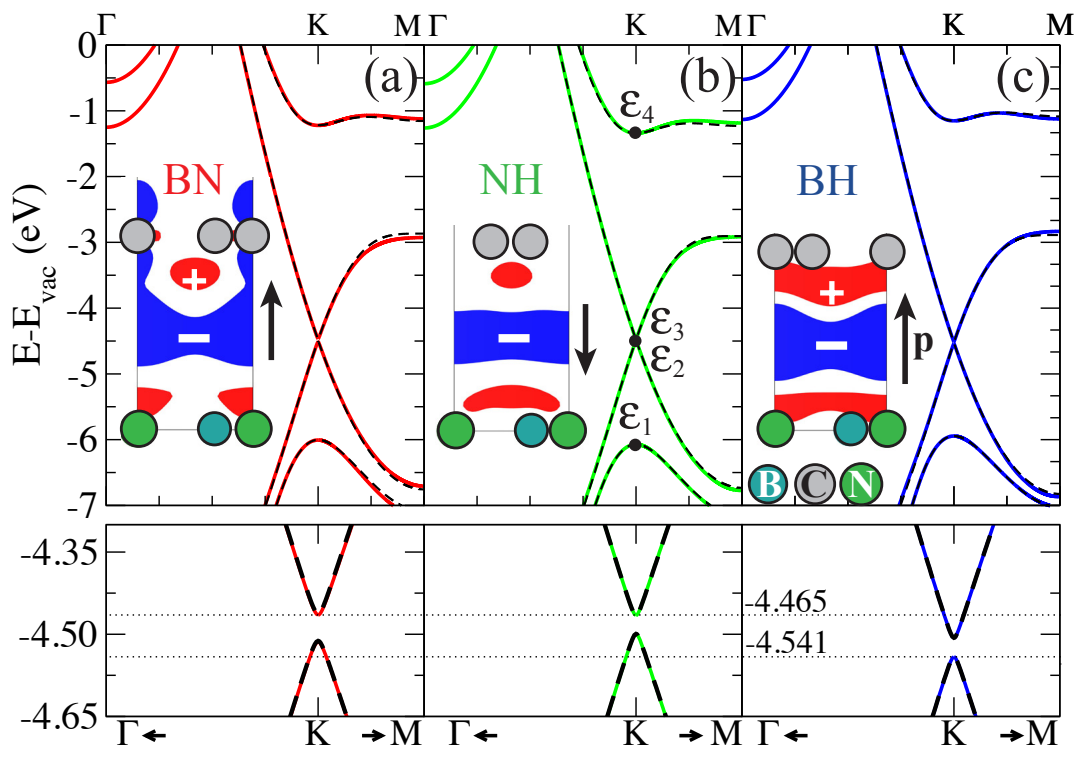

Figure 2.1: (Color) LDA band structures of graphene on $h$-BN for the (a), (b), and (c) commensurable structures, with respect to a common vacuum level. Solid and dashed lines give the DFT results and TB fits, respectively. The region around the Dirac point is enlarged in the panels below. The formation of an interface dipole is illustrated by the charge displacement $-e \Delta n$ in the $y z$ plane containing $\mathrm{B}, \mathrm{C}$ and $\mathrm{N}$ atoms. Blue and red indicate regions of negative and positive charge density, respectively, giving a dipole moment $\mathbf{p}$.

ite system into three parts: $H_{\overline{\mathrm{C}}}$ and $H_{\mathrm{BN}}$ for free-standing graphene and $h$-BN layers, respectively, and $H_{\text {int }}$ that describes the interaction between the two

$$
H_{\overline{\mathrm{C} \mid \mathrm{BN}}}=\left(\begin{array}{cc}
H_{\overline{\mathrm{C}}} & 0 \\
0 & H_{\mathrm{BN}}
\end{array}\right)+H_{\mathrm{int}} .
$$

We will see that $H_{\text {int }}$ has diagonal as well as off-diagonal elements. The reflection symmetry of ideally flat, free-standing graphene and $h$-BN monolayers allows us to classify states as either even $\left(s, p_{x}, p_{y}=s p^{2}\right)$ or odd $\left(p_{z}\right)$ under reflection in the plane. Because the matrix elements connecting even with odd states vanish, the Hamiltonian $H_{\mathrm{ML}}$ of a monolayer $(\mathrm{ML}=\overline{\mathrm{C}}$ or $\mathrm{BN})$ can be decomposed into blocks containing $p_{z}$ and $s p^{2}$ atomic orbitals

$$
H_{\mathrm{ML}}=\left(\begin{array}{cc}
H_{s p^{2}} & 0 \\
0 & H_{p_{z}}
\end{array}\right) .
$$



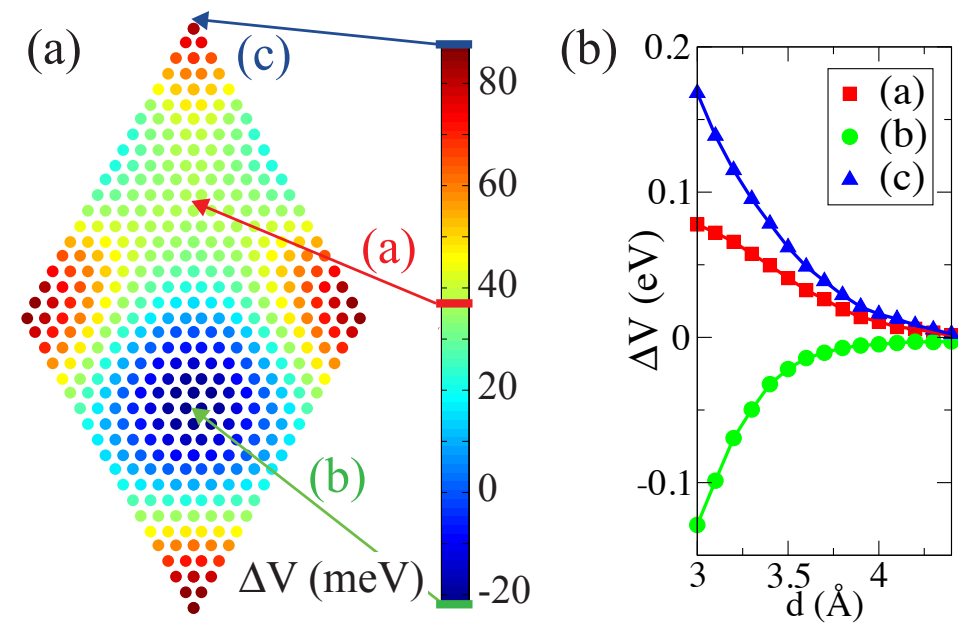

Figure 2.2: (Color) (a) The interface potential step $\Delta V$ as a function of the position $(x, y)$ of graphene with respect to $h$-BN, at the RPA equilibrium separations $d_{\mathrm{eq}}(x, y)$ obtained by interpolating the RPA results [45]. (b) Dependence of $\Delta V$ on $d$ for configurations $[(\mathrm{a})-(\mathrm{c})]$.

Since the Dirac cone in a single graphene layer arises from the interactions between $p_{z}$ orbitals, in first instance we only need $H_{\overline{\mathrm{C}} ; p_{z}}$ which can be written as

$$
H_{\overline{\mathrm{C}} ; p_{z}}=\left(\begin{array}{cc}
H_{\mathrm{C}} & H_{\mathrm{CC}^{\prime}} \\
H_{\mathrm{CC}^{\prime}}^{\dagger} & H_{\mathrm{C}^{\prime}}
\end{array}\right)
$$

where the indices $C$ and $C^{\prime}$ refer to the $A$ and $B$ sublattices of graphene. Since for a single graphene layer both carbon atoms in sublattices $\mathrm{A}$ and $\mathrm{B}$ are the same we have $H_{\mathrm{C}}=H_{\mathrm{C}^{\prime}}$. We parameterize $H_{\overline{\mathrm{C}} ; p_{z}}$ using two-center Slater-Koster form [10] with atomic orbitals as the basis for the Hamiltonian and overlap matrices. In this scheme the interaction between two $p_{z}$ orbitals on atoms connected by the in-plane vector $\mathbf{r}_{j}$ can be written as $(p p \pi)_{j} \equiv(p p \pi)\left(r_{j}\right)$, where $r_{j}$ is the distance between the two carbon atoms. In the Slater-Koster representation $H_{\overline{\mathrm{C}} ; p_{z}}$ can be written as

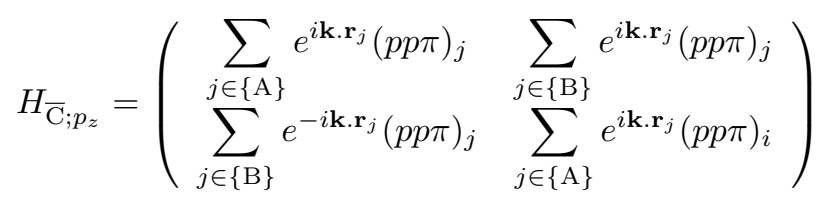

where $\{A\}(\{B\})$ is the set of carbon atoms of $\mathrm{A}(\mathrm{B})$-sublattice. The interaction integrals $(p p \pi)_{j}$ will be determined by fitting the TB bands to bands obtained from a first-principles calculation. $r_{j}$ with $j=1,2,3,4$ are the distances separating nearest neighbour, next nearest neighbour, third and fourth nearest neighbour atoms. The 


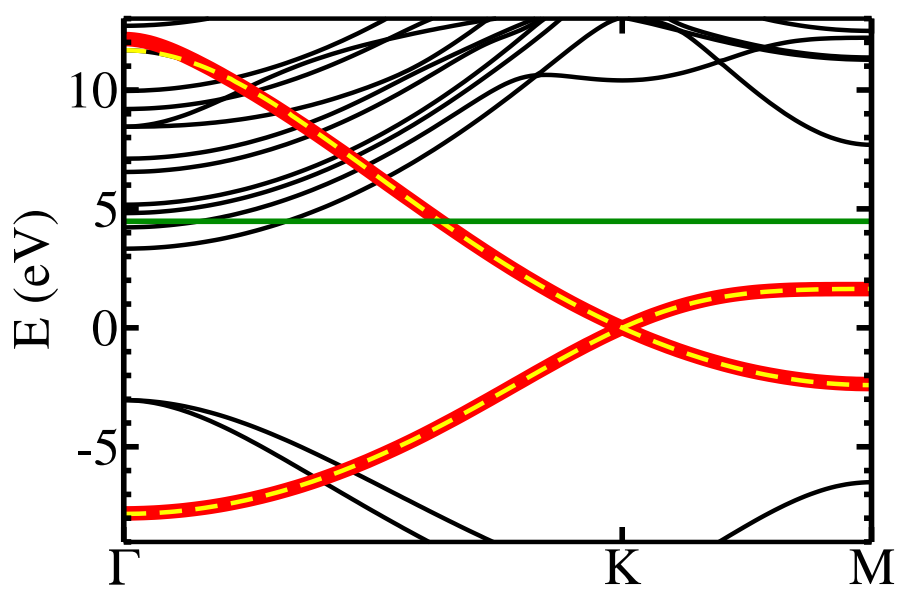

Figure 2.3: Full LDA band structure for a graphene monolayer. The $p_{z}$ bands calculated with LDA are highlighted in yellow (dashed curves) with the tight binding band highlighted in red (solid curve). The vacuum level is indicated by the horizontal green line. Above this level states are not localized normal to the graphene plane so that tight binding is not the most appropriate way of describing them.

overlap matrix will have the same form as the Hamiltonian only with different values of the hopping matrix elements.

Electronic structure codes like VASP $[58,59,9]$ that use a plane wave basis impose periodic boundary conditions. We simulate single layers by introducing a "vacuum" spacer layer between periodic images in the direction normal to the graphene plane. By choosing the corresponding lattice constant to be sufficiently large, the interaction between occupied states that are localized on a single graphene sheet with the periodic images can be made as small as required. However, states above the vacuum level will always "see" the periodic images and are poorly represented by a TB form. Our fitting procedure therefore only makes use of the electronic states below the vacuum level. As shown in Fig. 2.3 for an in-plane lattice constant of $a=2.445 \AA$, the tight binding bands can be nicely fit to the LDA band structure throughout the Brillouin zone. The fitting error was at most $1 \mathrm{meV}$ around the $\mathbf{K}$ point and the slope of the bands is accurately reproduced. The dependence of the values of the hopping parameters on $r_{j}$, shown in Table 2.1, indicates that we only need three shells of neighbours around a carbon atom in the graphene layer to describe the occupied part of the LDA band structure very satisfactorily. Since a $h$-BN layer is also flat, we can apply the same procedure to obtain $H_{\mathrm{BN} ; p_{z}}$. However, the absence of parity in this layer breaks the symmetry between the boron and nitrogen sublattices so that $H_{\mathrm{B}} \neq H_{\mathrm{N}}$; the on-site energies of boron and nitrogen are different and the hopping between boron atoms and between nitrogen atoms is different. The $h$-BN block of 


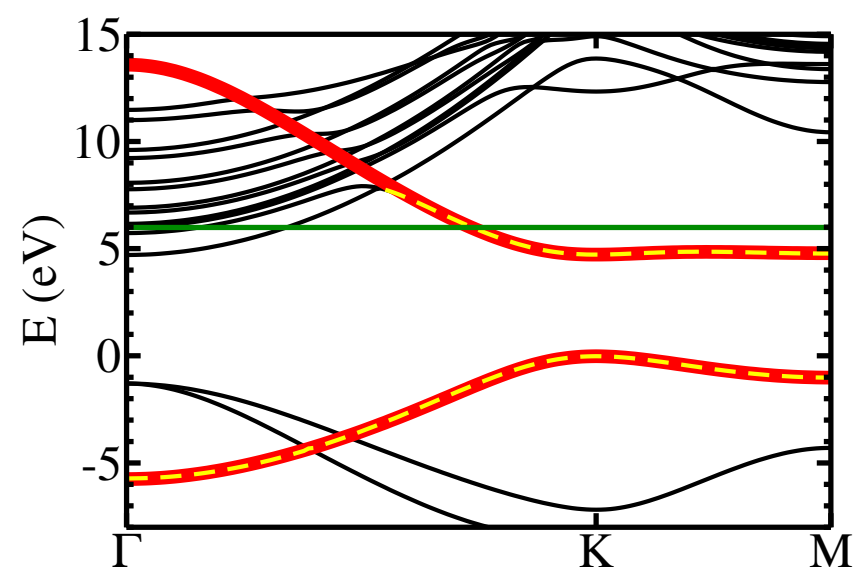

Figure 2.4: Same as Fig. 2.3 but for a $h$-BN monolayer. In the LDA, the minimum band gap of $\sim 4.5 \mathrm{eV}$ is underestimated, direct and occurs at the $\mathbf{K}$ point.

the Hamiltonian becomes

$$
H_{\mathrm{BN} ; p_{z}}=\left(\begin{array}{cc}
H_{\mathrm{B}} & H_{\mathrm{BN}} \\
H_{\mathrm{BN}}^{\dagger} & H_{\mathrm{N}}
\end{array}\right)
$$

The results of the tight binding parametrization of $H_{\mathrm{BN} ; p_{z}}$ given in Table 2.1 were obtained by fitting the corresponding LDA band structure shown in Fig. 2.4.

To parameterize the interaction between the graphene and $h$-BN layers, we begin by noting that the hopping interaction between the layers is short-range, each atom in one layer interacting with only a few neighbours in the other layer. In the SlaterKoster scheme [10], interactions are described in terms of orbitals with the angular momentum quantization axis chosen along the interatomic bond; the atomic orbitals are assumed to be separable, products of radial functions and spherical (or cubic) harmonics. Hopping matrix elements then depend only on the distance between the two atoms and the angular momentum about the bond axis. Transformations as a result of rotations are "canonical" and purely geometrical. For the planar structure of graphene and $h-\mathrm{BN}$, it is convenient to choose the $z$ axis normal to the plane. This means that in addition to needing to know the separation dependence of the $p p \sigma$ interaction that describes the interaction between a $\mathrm{C} p_{z}$ orbital immediately above e.g. a B $p_{z}$ orbital, we also need to know the separation dependence of the $p p \pi$ interaction; both are also needed to describe how the interaction is modified when the graphene and $h$-BN layers are displaced parallel to one another. The scheme could be extended to take account of strain by including variation of the in-plane lattice parameter.

The dipoles induced between the layers [61,52] change the environment surrounding each atom, adding additional terms to the interaction Hamiltonian $H_{\text {int }}$. 


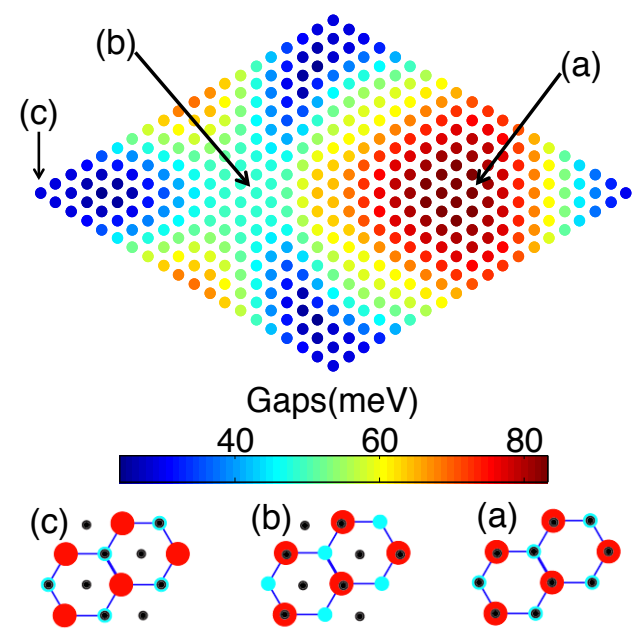

Figure 2.5: LDA band gaps of graphene on $h$-BN for many commensurable configurations derived from the TB model as a function of the in-plane displacement $\mathbf{r}=(x, y)$ for a fixed separation $d=3.4 \AA$ between planar graphene and $h$-BN sheets. The geometrical structure of the three most symmetric commensurable configurations labelled (a), (b), and (c) are sketched below where the black dots are carbon atoms, blue circles are boron atoms, and red circles are nitrogen atoms.

Since these terms do not depend on the wavevector, they should be included as diagonal elements in $H_{\text {int }}$. As a result the interaction part of the Hamiltonian is

$$
H_{\text {int }}=\left(\begin{array}{cccc}
V_{\mathrm{C}} & 0 & H_{\mathrm{CB}} & H_{\mathrm{CN}} \\
0 & V_{\mathrm{C}^{\prime}} & H_{\mathrm{C}^{\prime} \mathrm{B}} & H_{\mathrm{C}^{\prime} \mathrm{N}} \\
H_{\mathrm{CB}}^{\dagger} & H_{\mathrm{C}^{\prime} \mathrm{B}}^{\dagger} & V_{\mathrm{B}} & 0 \\
H_{\mathrm{CN}}^{\dagger} & H_{\mathrm{C}^{\prime} \mathrm{N}}^{\dagger} & 0 & V_{\mathrm{N}}
\end{array}\right)
$$

where $V_{\mathrm{C}}, V_{\mathrm{C}^{\prime}}, V_{\mathrm{B}}$, and $V_{\mathrm{N}}$ are the on-site terms representing the contribution of the dipole layer that depends on the local geometry [61,52]. The maximum overlap between atomic orbitals of the graphene layer with those of the $h$-BN layer is much smaller than the overlap of atomic orbitals in the graphene layer or in the $h$-BN layer. This reflects the weak binding between the two layers, the large layer separation, and the well documented small dispersion normal to the layers of bulk graphite or $h$-BN. For convenience, we assume that the interlayer overlap matrix is vanishingly small.

With these assumptions, the diagonal $V$ terms in Eq. 2.6 as well as the interlayer hopping parameters, labelled $\perp,(p p \sigma)_{\perp}(r)$ and $\left.(p p \pi)\right)_{\perp}(r)$ were determined by simultaneously fitting the results of (a large number of) LDA calculations for aligned, commensurable $(1 \times 1) \overline{\mathrm{C}} \mid h$-BN as a function of the separation $d$ between the layers and of an in-plane displacement $\mathbf{r}=(x, y)$ between the graphene and $h$-BN sheets. We were able to fit the band structure for all configurations to their corresponding 
Table 2.1: Hamiltonian and overlap matrix elements $(p p \pi)_{j} \equiv(p p \pi)\left(r_{j}\right)$ for graphene and $h$-BN monolayers with a lattice constant of $a=2.445 \AA$. The subscripts 1, 2, and 3 indicate nearest, next nearest, third nearest neighbour atoms separated by the distances $1.412,2.445$, and 2.823 respectively.

\begin{tabular}{lrrrrrrr}
\hline & \multicolumn{3}{c}{ Hamiltonian $(\mathrm{eV})$} & \multicolumn{3}{c}{ Overlap } \\
\cline { 2 - 8 }$i$ & on-site $\left(\varepsilon_{i}\right)$ & $(p p \pi)_{1}$ & $(p p \pi)_{2}$ & $(p p \pi)_{3}$ & $(p p \pi)_{1}$ & $(p p \pi)_{2}$ & $(p p \pi)_{3}$ \\
\hline $\mathrm{C}$ & 0.000 & -2.924 & -1.033 & -0.319 & 0.387 & 0.040 & 0.013 \\
$\mathrm{~B}$ & 3.207 & -2.333 & 0.020 & -0.294 & 0.211 & 0.111 & 0.051 \\
$\mathrm{~N}$ & -1.579 & -2.333 & 0.519 & -0.294 & 0.211 & -0.005 & 0.051 \\
\hline
\end{tabular}

LDA band structures around the $\mathbf{K}$ point with an accuracy of $1 \mathrm{meV}$ for band gaps indicating that the TB model is sufficiently robust. For a large aligned incommensurable supercell we will use these parameters to construct a global parameterization for the diagonal terms. The LDA band gaps of many configurations calculated with the above TB model are shown in Fig. 2.5 and the corresponding diagonal terms in Fig. 2.6. The diagonal terms are much smaller in magnitude than the hopping matrix elements and are short-range. For commensurable configurations, the overall dependence of the diagonal terms can be obtained by fitting the values shown in Fig. 2.6 with the Fourier expansion

$$
\begin{array}{r}
V_{\mathrm{C}}(\mathbf{r})=9.8+16 \cdot 6\left[\cos \mathbf{b}_{1} \cdot \mathbf{r}+\cos \mathbf{b}_{2} \cdot \mathbf{r}+\cos \left(\left(\mathbf{b}_{1}+\mathbf{b}_{2}\right) \cdot \mathbf{r}-0.55\right)\right] \\
V_{\mathrm{C}^{\prime}}(\mathbf{r})=-2.7+13\left[\cos \mathbf{b}_{1} \cdot \mathbf{r}+\cos \mathbf{b}_{2} \cdot \mathbf{r}+\cos \left(\left(\mathbf{b}_{1}+\mathbf{b}_{2}\right) \cdot \mathbf{r}-1.55\right)\right]
\end{array}
$$

where $\mathbf{b}_{1}$ and $\mathbf{b}_{2}$ are the primitive reciprocal lattice vectors of graphene and energies are in units of $\mathrm{meV}$. Shorter wavelengths (larger reciprocal lattice vectors) make a negligible contribution to the Fourier expansion of $V_{\mathrm{C}}$ and $V_{\mathrm{C}^{\prime}}$ and thus we ignored them in Eq. 2.7. Later we will use this observation in finding the Fourier expansion of $V$ terms of GW-corrected band structures.

The interlayer hopping parameters can be fitted to an exponential function of distance between atoms as

$$
\begin{array}{ll}
(p p \sigma)_{\perp}(r)= & -370 e^{-1.72(r-3.4)} \\
(p p \pi)_{\perp}(r)= & -316 e^{-1.46(r-3.4)}
\end{array}
$$

Here distances are in units of $\AA$ and energies are again in units of meV. The TB band structure for the (a) configuration of the $1 \times 1 \overline{\mathrm{C}} \mid h$-BN is compared to the corresponding LDA band structure in Fig. 2.6. Equation 2.7 is only valid for aligned commensurable configurations of the $\overline{\mathrm{C}} \mid h$-BN system. In Sect. 2.5.2, we will present an approximation for the angle-dependence of the diagonal terms for misaligned commensurable unit cells. The $1.8 \%$ lattice mismatch between graphene and $h$-BN layers results in supercells having more that 10000 atoms, much larger than the cor- 


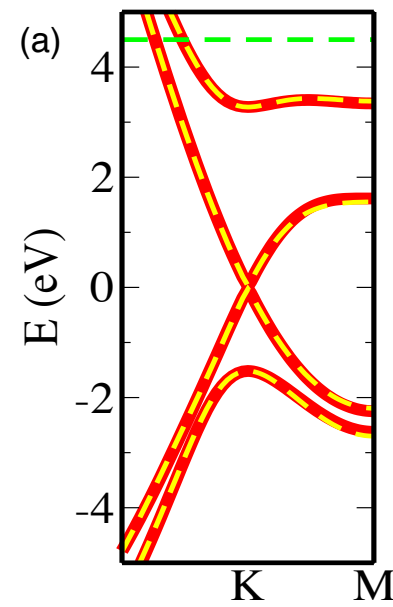

(b)

Figure 2.6: (a): Band structure of commensurable $\overline{\mathrm{C}} \mid h$-BN. The yellow-dashed curves are LDA bands and red curves are our TB bands. The dashed green line is the vacuum level. On-site energies of (b) A-sublattice carbon atoms, $V_{\mathrm{C}}(\mathbf{r})$, Eq. 2.7a and of (c) B-sublattice carbon atoms, $V_{\mathrm{C}^{\prime}}(\mathbf{r})$, Eq. $2.7 \mathrm{~b}$.

responding size of commensurable cells for the same misalignment angle. There are therefore no first-principles results for such big systems to be fitted to a TB model. Since the diagonal terms have been found to be short-range, and the magnitude of the hoppings in Eq. 2.8 are independent of configuration, in an incommensurable supercell they can be approximated by the local interactions as if the atom is in its corresponding local commensurable unit cell. This assumption is essential to parameterize the interlayer interactions. We will therefore use the functions in Eq. 2.7 for incommensurable supercells by scaling the reciprocal lattices $\mathbf{b}$ to the $h$-BN reciprocal lattice vectors.

\subsection{Quasiparticle corrections}

We can improve the TB model by using more accurate band structures. Though DFT calculations can usually predict the general trend of band structures [62], the KohnSham eigenvalues of DFT fail to describe the band gaps of insulators and semiconductors quantitatively [63]. This failure can be attributed to the fact that DFT eigenvalues are not quasi-particle energies of the system. This drawback can be remedied by including the electron-electron interactions within many-body perturbation theory where the so-called $G W$ approximation [64] yields significantly more accurate quasi-particle energies by comparison with experiment [65]. In this approximation, the exchange and correlation potentials are screened by a frequency-dependent dielectric function obtained using the random phase approximation (RPA). Starting 
with LDA Kohn-Sham (KS) orbitals [66] calculated for a cell containing two $h$-BN layers and a graphene layer, we use the $G W$ implementation in VASP [67], with 12 occupied and 52 empty bands and 50 points on the frequency grid for $1 \times 1$ aligned commensurable $\overline{\mathrm{C}} \mid h$-BN configurations. Interactions between periodic images in the $z$ direction lead to a dependence of the GW band gap on the cell size $D$ that we remove by linearly extrapolating the calculated gaps as a function of the inverse cell size to infinite separation [68].

We perform $G_{0} W_{0}$ calculations, where $G_{0}$ is the Green's function and $W_{0}$ is the screened potential calculated from LDA energy bands and wavefunctions. For the three most symmetric configurations of graphene on $h$-BN sketched in Fig. 2.5, we calculate the $G_{0} W_{0}$ corrections to the LDA band structures for a number of interlayer separations ranging from 3.35 to $3.55 \AA$. New $H_{\overline{\mathrm{C}}}, H_{\mathrm{BN}}$, diagonal and hopping terms of the interaction Hamiltonian $H_{\text {int }}$ for these three configurations were found by fitting a TB band structure to the corresponding $G W$ corrected band structures. The modified diagonal and hopping terms were then used to fit an in-plane periodic function like Eq. 2.7 used in the previous section. The local average of the on-site terms in Eq. 2.6, $\left(V_{\mathrm{C}}+V_{\mathrm{C}^{\prime}}\right) / 2$ varies about $200 \mathrm{meV}$, the difference $\left(V_{\mathrm{C}}-V_{\mathrm{C}^{\prime}}\right) / 2$ changes by around $150 \mathrm{meV}$ over the supercell. Because of the nonlocal nature of the screening of excitations, the $G_{0} W_{0}$ corrections depend on the size $D$ of the unit cell in the direction normal to the $\overline{\mathrm{C}} \mid h$-BN plane and converge slowly as a function of $D$. However, as shown in Fig. 2.7(b), this dependence is well approximated by $1 / D$ [68].

We can construct a simple theory to better understand the contributions of the electron-electron interactions in the graphene layer to the corrections to the band gaps calculated above. Since the graphene layer interacts weakly with the $h$-BN substrate and its Dirac cone is well separated in energy from the substrate $(h-\mathrm{BN})$ conduction and valence bands, we can assume that the main many-body corrections to the LDA band gaps of the substrate-supported graphene layer originate from electron-electron interactions in graphene itself; the $h$-BN substrate will make only a small contribution (in opening the LDA band gap and in the form of its static dielectric constant) to the $G W$-corrected band gaps. We thus represent $\overline{\mathrm{C}} \mid h-\mathrm{BN}$ as a free-standing graphene layer with a gap when we study the origin of the $G W$ corrections to the low-energy bands around the $\mathbf{K}$ point. With this assumption, we introduce a two-band model comprising only the unperturbed bands of the gapped graphene layer and write its Hamiltonian $H_{\overline{\mathrm{C}}, \Delta}$ as

$$
H_{\overline{\mathrm{C}}, \Delta}=\left(\begin{array}{cc}
\Delta & k e^{i \theta_{k}} \\
k e^{-i \theta_{k}} & -\Delta
\end{array}\right)
$$

where $\Delta$ is half of the LDA gap (of some specific configuration of the $\overline{\mathrm{C}} \mid h$-BN system), $\mathbf{k}$ is the wavevector, and we have set $\hbar v_{F}=1$. Using the solutions of the above Hamiltonian, we can calculate the RPA density-density response function [69] of the 


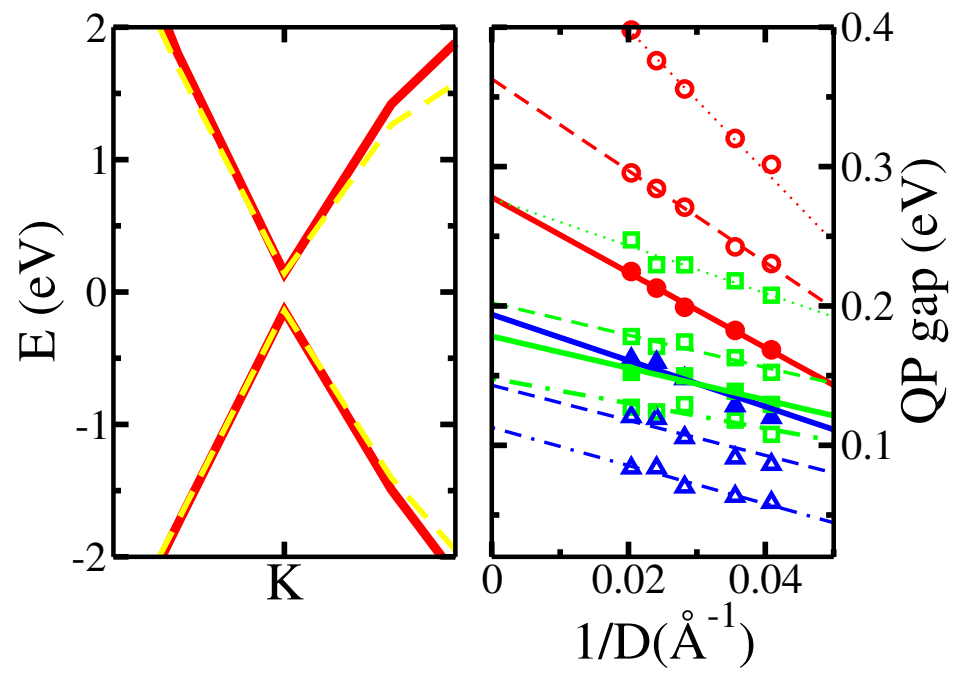

Figure 2.7: (a) Quasiparticle (QP) band structure of $\overline{\mathrm{C}} \mid h$-BN close to the $\mathbf{K}$ point. The yellow-dashed curves were obtained using the $G_{0} W_{0}$ approximation, the red curves are our TB bands. (b) QP band gaps opened at the Dirac point as a function of the inverse cell size for the configurations sketched in Fig. 2.5 labelled (a) red circles (b) green squares (c) blue triangles. The solid lines correspond to graphene at the RPA equilibrium distances [45]. The dotted, dashed, and dashed-dotted lines correspond to graphene- $h$-BN separations of $3.35,3.45$, and $3.55 \AA$, respectively.

gapped graphene layer using

$$
\Pi_{o}(\mathbf{q}, \omega)=\sum_{\mu, \mu^{\prime}} \int \frac{d^{2} k}{2 \pi^{2}} \frac{n_{F}\left(\varepsilon_{\mathbf{k} \mu}\right)-n_{F}\left(\varepsilon_{\mathbf{k}+\mathbf{q}, \mu^{\prime}}\right)}{\omega+\varepsilon_{\mathbf{k} \mu}-\varepsilon_{\mathbf{k}+\mathbf{q}, \mu^{\prime}}+i \delta} f_{\mu \mu^{\prime}}(\mathbf{k}, \mathbf{k}+\mathbf{q})
$$

where $\mu$ and $\mu^{\prime}$ refer to the occupied (+) and unoccupied (-) states, $n_{F}$ is the occupation number of the bands, $\omega$ is the frequency, $\mathbf{q}$ is a wavevector, $\delta$ is an infinitesimal small real number, and $f_{\mu \mu^{\prime}}(\mathbf{k}, \mathbf{k}+\mathbf{q})$ is the overlap of the wave functions at $\mathbf{k}$ and $\mathbf{k}+$ q. From Eq. 2.9 we have

$$
f_{\mu \mu^{\prime}}(\mathbf{k}, \mathbf{k}+\mathbf{q})=\frac{1}{2}\left(1+\mu \mu^{\prime} \frac{k^{2}+k q \cos \theta_{k, q}+\Delta^{2}}{\varepsilon_{\mathbf{k}} \varepsilon_{\mathbf{k}+\mathbf{q}}}\right)
$$

where $\varepsilon_{k}=\sqrt{k^{2}+\Delta^{2}}$ is an eigenvalue of the Hamiltonian (2.9). The imaginary part of the polarization function is

$$
\mathfrak{I} \Pi_{o}(\mathbf{q}, \omega)=-\frac{q^{2}}{4} \frac{\omega^{2}+4 \Delta^{2}-q^{2}}{\left(\omega^{2}-q^{2}\right)^{3 / 2}} \Theta\left(\omega-\sqrt{q^{2}+4 \Delta^{2}}\right) .
$$

where $\Theta(x)$ is the step function, zero for negative $x$ and one for positive $x$. The 


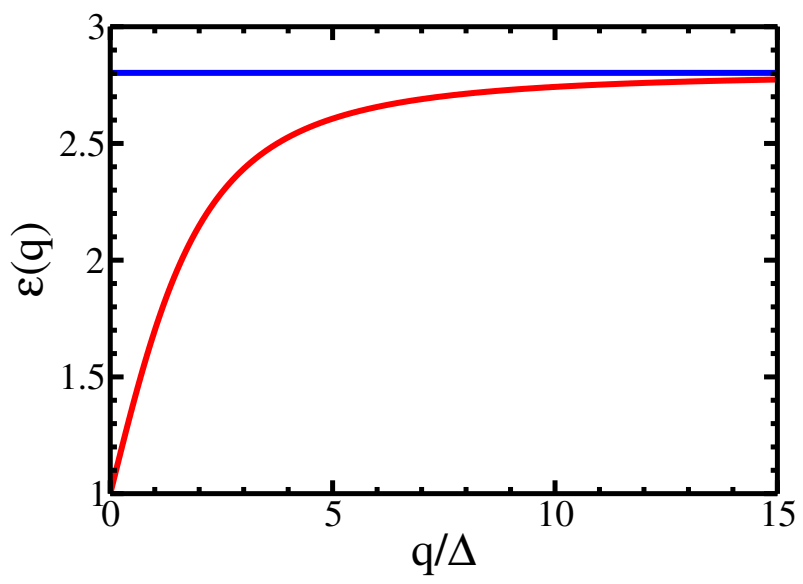

Figure 2.8: The static dielectric function as a function of the wavevector $\mathrm{q}$ for gapless (blue line) and gapped (red line) graphene.

real part of $\Pi_{o}$ can be derived from the imaginary part by using the Kramers-Kronig relation

$$
\begin{array}{r}
\Re \Pi_{o}(\mathbf{q}, \omega)=-q^{2} \frac{\Theta\left(\sqrt{q^{2}+4 \Delta^{2}}-\omega^{2}\right)}{4}\left[\frac{2 \Delta}{q^{2}-\omega^{2}}+\right. \\
\left.\frac{q^{2}-\omega^{2}-4 \Delta^{2}}{\left(q^{2}-\omega^{2}\right)^{3 / 2}}\left(1-\frac{2}{\pi} \tan ^{-1} \frac{2 \Delta}{\sqrt{q^{2}-\omega^{2}}}\right)\right] .
\end{array}
$$

The screened electron-electron interaction of gapped graphene in the RPA approximation is

$$
W(\mathbf{q}, \omega)=\frac{V(\mathbf{q})}{1-V(\mathbf{q}) \Pi_{o}(\mathbf{q}, \omega)}
$$

where $V(\mathbf{q})=\frac{2 \pi e^{2}}{\kappa q}$ is the Fourier transform of the Coulomb potential in two dimensions and $\kappa$ is the dielectric constant of the environment which is the average of dielectric constant of vacuum $(\kappa=1)$ and $h$-BN layer $(\kappa \simeq 2.8)$ [61]. The denominator in Eq. 2.14 is the RPA dielectric function. The static dielectric functions for gapless (blue line; constant) and gapped (red line) graphene are shown in Fig. 2.8. The gapped static dielectric function is seen to approach that of gapless graphene asymptotically for large wavevectors. The self-energy in the $G_{0} W$ approximation is

$$
\Sigma_{\mu^{\prime} \lambda}(\mathbf{k}, z)=-k_{B} T \sum_{\mathbf{q} \nu_{n} \mu} f_{\mu^{\prime} \mu \lambda}(\mathbf{k}, \mathbf{k}+\mathbf{q}) G_{0 \mu}\left(\mathbf{k}+\mathbf{q}, z+i \nu_{n}\right) W\left(\mathbf{q}, i \nu_{n}\right)
$$

where $z$ is the complex frequency, $\lambda$ is the chirality index, $\nu_{n}$ are Matsubara frequen- 

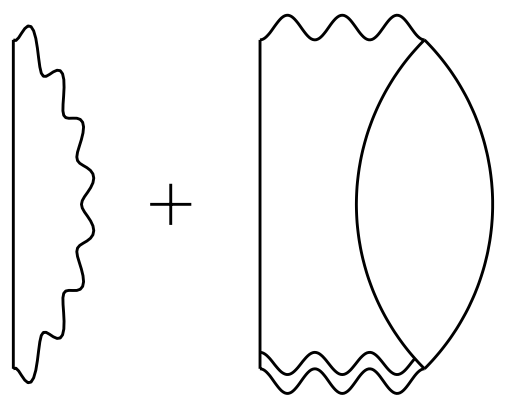

Figure 2.9: Self-energy diagrams in the $G_{0} W$ approximation. The first diagram is the Hartree self-energy, the second diagram is a sum of an infinite number of diagrams. The straight vertical lines representation the Green function $G_{0}$, the wavy lines represent the electron-electron interaction.

cies,

$$
f_{\mu^{\prime} \mu \lambda}(\mathbf{k}, \mathbf{k}+\mathbf{q})=\left\langle\mu^{\prime} \mathbf{k} \mid \mu, \mathbf{k}+\mathbf{q}\right\rangle\langle\mu, \mathbf{k}+\mathbf{q} \mid \lambda \mathbf{k}\rangle
$$

and we have transformed the convolution integral in self-energy to a summation over Matsubara frequencies in Eq. 2.15. The imaginary part of the self-energy can be written as:

$$
\mathfrak{I} \Sigma_{\lambda}(\omega)=r_{s} \int d \mathbf{q}\left(1+\lambda \frac{\Delta}{\sqrt{\Delta^{2}+q^{2}}}\right) \mathfrak{I}\left(\frac{1}{\varepsilon\left(\mathbf{q}, \omega-\varepsilon_{q}\right)}\right) \Theta\left(\omega-\varepsilon_{q}\right)
$$

where $\Sigma_{\lambda}(\omega)$ is the diagonal matrix element of $\Sigma$ at $\mathbf{k}=\mathbf{K}$ where the minimum band gap occurs, $r_{s}=e^{2} / \kappa \hbar v_{F}$ is the interaction coupling parameter in graphene that determines the ratio of the electron-electron potential to the kinetic energy. Using the Kramers-Kronig relation for self-energy, we can write the real part as

$$
\mathfrak{R} \Sigma_{\lambda}(\omega)=\mathfrak{R} \Sigma_{\lambda}(\infty)+\frac{2}{\pi} P \int_{3 \Delta}^{2 \Lambda} \frac{\omega^{\prime} \mathfrak{I} \Sigma_{\lambda}\left(\omega^{\prime}\right)}{\omega^{\prime 2}-\omega^{2}} d \omega^{\prime}
$$

where the lower limit of the integral is chosen in such a way that the imaginary part of the dielectric function can be non-zero. This happens if the frequency is bigger than the gap $(2 \Delta)$ and because the frequency argument in Eq. 2.17 is $\omega-\varepsilon_{q}$ then $2 \Delta+\varepsilon_{q} \leq \omega$ and since $\Delta \leq \varepsilon_{q}$ then we can conclude that the lower bound of the integral in Eq. 2.18 should be $3 \Delta \leq \omega$. The first term in the above equation, called the Hartree self-energy correction and corresponding to the first diagram in Fig.2.9, overestimates the quasi-particle corrections. The second term, corresponding to the second diagram in Fig. 2.9, is a sum of an infinite number of bubble diagrams of RPA in the $G_{0} W$ correction and is negative. The frequency cut-off in Eq. 2.18, the upper limit in the integral, is determined by the graphene bandwidth. In the LDA, the width of the $\pi$ bands is about $18 \mathrm{eV}$ (Fig. 2.3) so the cut-off will be $\Lambda \sim 9 \mathrm{eV}$. 
The Hartree corrected band gap for the two-band gapped graphene model can be analytically evaluated as

$$
\Delta_{\mathrm{H}}=\Delta_{\mathrm{LDA}}\left(1+r_{s} \ln \frac{2 \Lambda}{\Delta_{\mathrm{LDA}}}\right) .
$$

The second term can be approximated as

$$
\Delta^{(2)} \approx-\Delta_{\mathrm{LDA}} \frac{r_{s}^{2}}{2} \ln \frac{2 \Lambda}{3 \Delta_{\mathrm{LDA}}}
$$

To determine the energy correction using the self-energy, we use the relation

$$
\varepsilon_{n k}=\varepsilon_{n k}^{(0)}+\mathfrak{R} \Sigma\left(k, \varepsilon_{n k}\right)
$$

which should be solved self-consistently. We can expand the self-energy about the uncorrected energies $\varepsilon_{n k}^{(0)}$ as

$$
\Sigma\left(k, \varepsilon_{n k}\right) \simeq \Sigma\left(k, \varepsilon_{n k}^{(0)}\right)+\left.\frac{\partial \Sigma(k, \omega)}{\partial \omega}\right|_{\omega=\varepsilon_{n k}^{(0)}}\left(\varepsilon_{n k}-\varepsilon_{n k}^{(0)}\right)
$$

and this is the $G_{0} W_{0}$ self-energy correction. Using this approximation, the quasiparticle energies will be

$$
\varepsilon_{n k} \simeq \varepsilon_{n k}^{(0)}+\frac{\Re \Sigma\left(k, \varepsilon_{n k}^{(0)}\right)}{1-\left.\frac{\partial \Re \Sigma(k, \omega)}{\partial \omega}\right|_{\omega=\varepsilon_{n k}^{(0)}}}
$$

Fig. 2.10 shows the Hartree-corrected band gaps along with the total correction calculated numerically on the basis of the above two-band model. The agreement between the results of the two-band model and the first-principles $G_{0} W_{0}$ band gaps indicates that the main contribution to the many body correction for the graphene band gap comes from the screening of the electron-electron interactions in the graphene itself, and is independent of the specific configuration of the $\overline{\mathrm{C}} \mid h$-BN system.

\subsection{Solving the Hamiltonian}

The weak interlayer interaction in the $\overline{\mathrm{C}} \mid h$-BN system means there is no (strong) preference for aligning the crystal axes of graphene and $h$-BN. We consider the aligned incommensurable case and misaligned cases separately.

\subsubsection{Aligned incommensurable graphene $\mid h-\mathrm{BN}$}

Aligned incommensurable configurations of $\overline{\mathrm{C}} \mid h$-BN can be modelled using the rational approximant

$$
\frac{N_{\overline{\mathrm{C}}}}{N_{\mathrm{BN}}}=\frac{a_{\mathrm{BN}}}{a_{\overline{\mathrm{C}}}}=1.018 \approx \frac{56}{55}
$$




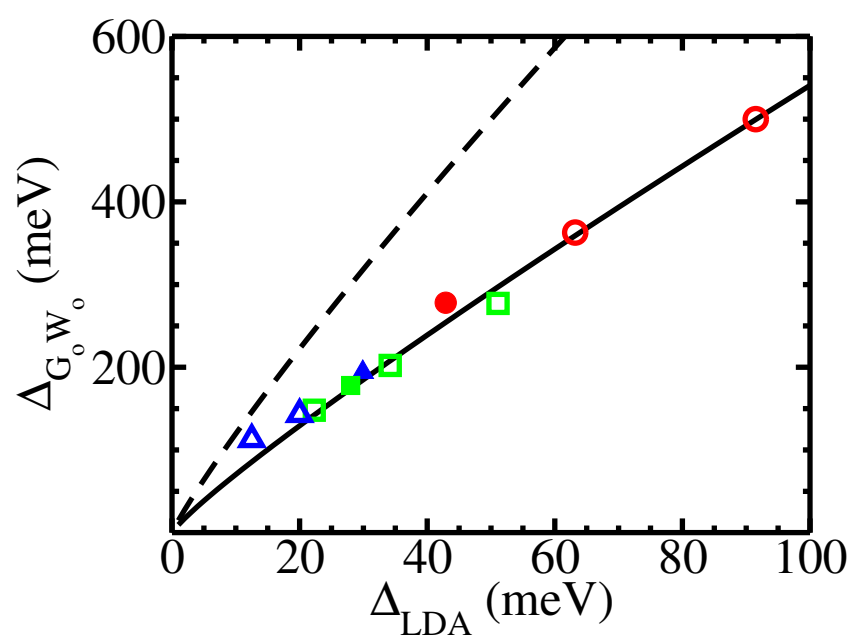

Figure 2.10: The $G_{0} W_{0}$ corrected band gaps of graphene on $h$-BN as a function of the LDA band gaps. The blue triangles, red circles and green squares are the values obtained for different configurations and layer separations $d$ by extrapolating the data in Fig. 2.7 to $D \rightarrow \infty$. The dashed curve is the Hartree quasiparticle gap obtained using Eq. 2.19, the solid curve is the band gap obtained by solving Eq. 2.18 numerically for the self-energy and using Eq. 2.23.

and a periodic supercell containing $N_{\overline{\mathrm{C}}} \times N_{\overline{\mathrm{C}}}$ unit cells of graphene and $N_{\mathrm{BN}} \times N_{\mathrm{BN}}$ unit cells of $h$-BN. The resulting supercell has dimensions of $\sim 14 \mathrm{~nm}$, in agreement with experimental observations of moiré patterns $[43,44]$. Diagonalizing the corresponding TB Hamiltonian for flat graphene separated by $d=3.4 \AA$ from flat $h$-BN [45] using the parameterization explained in the previous section results in gaps of $\sim 4 \mathrm{meV}$ for the LDA parametrization and $\sim 5 \mathrm{meV}$ for the $G_{0} W_{0}$ parametrization.

For commensurable aligned $\overline{\mathrm{C}} \mid h-\mathrm{BN}$, the ACFDT RPA calculations predict different equilibrium separations $d_{\mathrm{eq}}^{\mathrm{RPA}}=3.55,3.50$ and $3.35 \AA$ for the (a), (b) and (c) configurations [45]. As a result of the $1.8 \%$ lattice mismatch, carbon atoms in a graphene sheet aligned incommensurably with a $h$-BN substrate see different environments that locally can be approximated by commensurable graphene on $h$-BN displaced in plane by some amount. Because the perodicity of $14 \mathrm{~nm}$ is so large, it is energetically favourable for the separation between the graphene and $h$-BN layers to then vary locally giving rise to a wavy interface. This height modulation can be important for determining the electronic and transport properties of $\overline{\mathrm{C}} \mid h$-BN [70]. The interlayer separation can be determined for a superlattice by assuming that the height variation $d(\mathbf{r})$ (in $\AA$ ) is smooth over the supercell

$$
d_{\mathrm{eq}}^{\mathrm{RPA}}(\mathbf{r})=3.47-0.043\left[\cos \mathbf{B}_{1} \cdot \mathbf{r}+\cos \mathbf{B}_{2} \cdot \mathbf{r}+\cos \left(\left(\mathbf{B}_{1}+\mathbf{B}_{2}\right) \cdot \mathbf{r}+0.74\right)\right]
$$

where $\mathbf{B}_{1}$ and $\mathbf{B}_{2}$ are the reciprocal lattice vectors of the supercell. Taking this height 
variation into account in the LDA parametrization of the TB Hamiltonian results in a gap of $5 \mathrm{meV}$ which is only slightly larger than the $4 \mathrm{meV}$ gap we find for the corresponding flat structure. More importantly, this wavy structure increases the $G_{0} W_{0}$ corrected band gap of flat $\overline{\mathrm{C}} \mid h$-BN from $5 \mathrm{meV}$ to $32 \mathrm{meV}$ at the $\mathbf{K}$ point, a value in good agreement with experiment [48].

There are two experimental situations that can lead to the absence of gap opening in $\overline{\mathrm{C}} \mid h$-BN. The first is when graphene and $h$-BN are not aligned; this will be discussed in detail in the next section. The second is when graphene is sandwiched between two $h$-BN layers where the topmost $h$-BN layer may suppress the lateral height modulation caused by the substrate $h$-BN, reducing the symmetry breaking potential and translating into a reduction in the band gap.

\subsubsection{Misaligned incommensurable graphene $\mid h-\mathbf{B N}$}

Because of the weak interlayer interaction, the graphene layer can be misaligned with respect to the underlying $h$-BN layer as observed experimentally [44]. The infinite number of possible misalignment angles is countable and each angle corresponds to a finite supercell whose size depends on the rotation angle [71]. Extending the formulation developed to study graphene $\overline{\mathrm{C}} \mid \overline{\mathrm{C}}$ bilayers [71] to include incommensurability in the $\overline{\mathrm{C}} \mid h$-BN system, we derive the following general condition for finding rotated supercells

$$
m_{1} \mathbf{a}_{1}^{*}\left(\frac{\theta}{2}\right)+m_{2} \mathbf{a}_{2}^{*}\left(\frac{\theta}{2}\right)=n_{1} \mathbf{a}_{1}\left(\frac{-\theta}{2}\right)+n_{2} \mathbf{a}_{2}\left(\frac{-\theta}{2}\right)
$$

where $\mathbf{a}_{1}(\theta)$ and $\mathbf{a}_{2}(\theta)\left(\mathbf{a}_{1}^{*}(\theta)\right.$ and $\left.\mathbf{a}_{2}^{*}(\theta)\right)$ are rotated lattice vectors of the graphene $\left(h\right.$-BN) layer. The conditions that must be satisfied by the integers $n_{1(2)}$ and $m_{1(2)}$ can be simplified into the Diophantine equations

$$
N_{\mathrm{BN}} U(-p, q)\left(\begin{array}{c}
m_{1} \\
m_{2}
\end{array}\right)=N_{\overline{\mathrm{C}}} U(p, q)\left(\begin{array}{l}
n_{1} \\
n_{2}
\end{array}\right)
$$

where $U$ can be written as

$$
U(p, q)=\left(\begin{array}{cc}
-p+3 q & -2 p \\
2 p & p+3 q
\end{array}\right)
$$

and $p$ and $q$ are two arbitrary integers that determine the rotation angle through the condition

$$
\theta=\cos ^{-1}\left(\frac{3 q^{2}-p^{2}}{3 q^{2}+p^{2}}\right)
$$

This equation has an infinite number of solutions. For commensurable graphene and $h$-BN, the smallest $\overline{\mathrm{C}} \mid h$-BN unit cell found from this equation for $p=1$ and $q=3$ contains 7 unit cells of each layer and is rotated through $\theta \approx 21.8^{\circ}$.

Using $h$-BN primitive unit cells, the graphene layer can be partitioned so that 
each carbon atom will be located in only one primitive unit cell of the $h$-BN substrate. The direction vector connecting a given carbon atom at a position $\mathbf{r}$ to its nearest neighbour carbon atom inside the same $h$-BN cell makes some angle $\phi$ with the $h$-BN primitive lattice vectors. This configuration cannot be described as a simple translation of a known commensurably aligned $\overline{\mathrm{C}} \mid h$-BN configuration so Eq. 2.7 cannot be used to determine the diagonal terms of the TB Hamiltonian. We therefore introduce a simple interpolation $V(\mathbf{r}, \phi)$ for the diagonal terms in the TB Hamiltonian of a commensurable aligned $\overline{\mathrm{C}} \mid h$-BN configuration to give an equation for the on-site terms $V(\mathbf{r}, \phi)$ of the corresponding rotated configuration. The three-fold symmetry of the local environment of each atom within the supercell keeps $V(\mathbf{r}, \phi)$ of the atoms unchanged under rotations of 120 degrees. Therefore, we can expand $V(\mathbf{r}, \phi)$ by using terms with three-fold symmetry. The simplest function will be

$$
V(\mathbf{r}, \phi)=V(\mathbf{r}, 0)+\frac{V(\mathbf{r}, \pi / 3)-V(\mathbf{r}, 0)}{2}(1-\cos 3 \phi)
$$

where $V(\mathbf{r}, 0)$ and $V(\mathbf{r}, \pi / 3)$ are the diagonal terms of the two commensurable configurations which can be found from Eq. 2.7. To use Eq. 2.30 for a supercell, we first find the $h$-BN primitive cell to which a carbon atom belongs and the vector $\mathbf{r}$, we then find the relative angle $\phi$ of that carbon and its nearest neighbour carbon atom inside the same $h$-BN primitive cell, and then use Eq. 2.30 to determine the onsite potential term.

This can be illustrated using the (b) and (c) configurations of Fig. 2.5. We observe that these configurations are related by translations along the cell diagonal; starting from configuration (c), we can displace the graphene layer to reach configuration (b). We also see that configuration (b) is obtained by rotating configuration (c) through 60 degrees about the center of its $h$-BN hexagon and vice-versa; however, this rotation also interchanges the sublattices. The diagonal terms of the carbon atoms at the center of $h$-BN hexagons of configurations (b) and (c) are known from section III. Now, the on-site term $V(\mathbf{r}, \phi)$, where $\mathbf{r}$ is the position of the carbon atom, for the atom at the center of a $h$-BN hexagon of any rotated configuration can be written in terms of the on-site terms of the carbon atoms located at the center of the $h$-BN hexagons of configurations (b) and (c). Here the on-site term of the carbon atom in the center of the hexagon of configuration (c) is $V(\mathbf{r}, 0)$, and the on-site term of configuration (b) is $V(\mathbf{r}, \pi / 3)$.

If we apply the above modification of the TB model to configurations rotated through large angles, no band gap is found. For the commensurable rotated case, this could be confirmed by performing explicit DFT calculations for these configurations. For large rotation angles, the band structure of the substrate-supported graphene layer looks like that of a free-standing graphene layer near the $\mathbf{K}$ point. This implies that a large rotation of the $\overline{\mathrm{C}} \mid h$-BN system decouples the graphene layer from its underlying substrate.

On the other hand, for small rotation angles, the system should have a band gap close to $32 \mathrm{meV}$, the value we found in the previous section for perfectly aligned 
incommensurable $\overline{\mathrm{C}} \mid h$-BN at the $\mathbf{K}$ point. A transition from a conducting $\overline{\mathrm{C}} \mid h$-BN to an insulating $\overline{\mathrm{C}} \mid h$-BN must therefore occur. The smallest angle we have studied with the TB model is about 4 degrees for which no band gap was found making this an upper limit for the transition angle. Experimental reports of a critical angle of $\sim 1$ degree [56] also suggest that a commensurable-incommensurable transition occurs in the $\overline{\mathrm{C}} \mid h$-BN system. Although the $30 \mathrm{meV}$ gap in that report was attributed to strain in the graphene layer as it adapts itself to the $h$-BN layer [56], our $G W$ calculations suggest that the band gaps of aligned commensurable regions in the supercell should be as large as several hundred meV. We suggest that the $30 \mathrm{meV}$ gap is actually the global gap that originates from incommensurable regions of the moiré patterns. Since a rotation of the commensurable system is found to suppress the gap, we conclude that the critical angle for rotated incommensurable system will also be small. Therefore, a small misalignment of layers will completely destroy this gap and we argue that this is the reason that the initial experiments on the $\overline{\mathrm{C}} \mid h$-BN system[43, 44] did not report any gap.

Any rotation larger than the critical angle will significantly change the surrounding of atoms and destroy the band gap, making the modulating potential the dominant potential in the system and charge redistribution possible. This charge redistribution will lead to the formation of "intrinsic" electron-hole puddles in the supercell [72].

\subsection{Löwdin downfolding and effective Hamiltonian}

In this section, we present an effective Hamiltonian for $\overline{\mathrm{C}} \mid h$-BN [50]. The wavefunction will be a mixture of the wavefunctions of the graphene and $h$-BN layers. Using Löwdin downfolding, we can eliminate the $h$-BN wave function in the Schrödinger equation for $\overline{\mathrm{C}} \mid h-\mathrm{BN}$

$$
\left(\begin{array}{cc}
\tilde{H}_{\overline{\mathrm{C}}} & H_{h} \\
H_{\mathrm{h}}^{\dagger} & \tilde{H}_{\mathrm{BN}}
\end{array}\right)\left(\begin{array}{c}
\psi_{\overline{\mathrm{C}}} \\
\psi_{\mathrm{BN}}
\end{array}\right)=\varepsilon\left(\begin{array}{cc}
O_{\overline{\mathrm{C}}} & 0 \\
0 & O_{\mathrm{BN}}
\end{array}\right)\left(\begin{array}{c}
\psi_{\overline{\mathrm{C}}} \\
\psi_{\mathrm{BN}}
\end{array}\right)
$$

where $\tilde{H}_{\overline{\mathrm{C}}}$ and $\tilde{H}_{\mathrm{BN}}$ contain the diagonal parts of the interaction matrix $H_{\text {int }}$ given in Eq. 2.6, $H_{h}$ is the off-diagonal "hopping" part of $H_{\text {int }}$ and $O_{\overline{\mathrm{C}}(B N)}$ is the graphene $(h$-BN) overlap matrix. We can absorb all the effects of the substrate into an effective interaction Hamiltonian $H_{e}$

$$
H_{e}=\tilde{H}_{\overline{\mathrm{C}}}+H_{h}\left(\varepsilon O_{\mathrm{BN}}-\tilde{H}_{\mathrm{BN}}\right)^{-1} H_{h}^{\dagger}
$$

Since we are interested in energies close to the Dirac point of graphene that are small compared to the eigenstates of $H_{\mathrm{BN}}$, we can formally expand $H_{e}$ as a function of the graphene eigenenergies

$$
H_{e} \approx \tilde{H}_{\overline{\mathrm{C}}}-H_{h} H_{\mathrm{BN}}^{-1} H_{\mathrm{h}}^{\dagger}-\varepsilon H_{\mathrm{h}} \tilde{H}_{\mathrm{BN}}^{-1} O_{\mathrm{BN}} \tilde{H}_{\mathrm{BN}}^{-1} H_{\mathrm{h}}^{\dagger}
$$


We can also expand the eigenenergies $(\varepsilon)$ as a function of the interaction strength $\left(O\left(H_{h}\right)\right)$. From Eq. 2.33 it is clear that $\varepsilon \approx O\left(H_{h}^{2}\right)$ so the third term in Eq. 2.33 is of the fourth order $\left(O\left(H_{h}^{4}\right)\right)$ and therefore in calculations to the second order can be neglected. Our parametrization in section II showed that these interactions are small compared to the $h$-BN eigenenergies. The effective Hamiltonian corrected to the second order for the graphene layer is therefore

$$
H_{e}^{(2)}=\tilde{H}_{\overline{\mathrm{C}}}-H_{h} \tilde{H}_{\mathrm{BN}}^{-1} H_{\mathrm{h}}^{\dagger}
$$

$H_{e}^{(2)}$ includes the effective modulating potential $V$, an effective local symmetrybreaking potential $\Delta$ and a "vector potential" $\mathbf{A}$. $V$ is locally related to the mass terms and hopping parameters

$$
V=\frac{1}{2}\left[V_{\mathrm{C}}+V_{\mathrm{C}^{\prime}}+\frac{\left|H_{\mathrm{CB}}\right|^{2}+\left|H_{\mathrm{C}^{\prime} \mathrm{B}}\right|^{2}}{\varepsilon_{\mathrm{B}}}+\frac{\left|H_{\mathrm{CN}}\right|^{2}+\left|H_{\mathrm{C}^{\prime} \mathrm{N}}\right|^{2}}{\varepsilon_{\mathrm{N}}}\right]
$$

where $\varepsilon_{B}$ and $\varepsilon_{N}$ are onsite energies of boron and nitrogen atoms respectively. The hopping terms modify the modulating potential $\left(V_{\mathrm{C}}+V_{\mathrm{C}^{\prime}}\right) / 2$ of the interface dipole(s). The local sublattice symmetry-breaking potential is also modified by hopping

$$
\Delta=\frac{1}{2}\left[V_{\mathrm{C}}-V_{\mathrm{C}^{\prime}}+\frac{\left|H_{\mathrm{CB}}\right|^{2}-\left|H_{\mathrm{C}^{\prime} \mathrm{B}}\right|^{2}}{\varepsilon_{\mathrm{B}}}+\frac{\left|H_{\mathrm{CN}}\right|^{2}-\left|H_{\mathrm{C}^{\prime} \mathrm{N}}\right|^{2}}{\varepsilon_{\mathrm{N}}}\right]
$$

The components $\left(A_{x}, A_{y}\right)$ of the vector potential which only contain the hopping interactions are given by

$$
A_{x}+i A_{y}=\frac{H_{\mathrm{CB}} H_{\mathrm{C}^{\prime} \mathrm{B}}^{\dagger}}{\varepsilon_{\mathrm{B}}}+\frac{H_{\mathrm{CN}} H_{\mathrm{C}^{\prime} \mathrm{N}}^{\dagger}}{\varepsilon_{\mathrm{N}}}
$$

The first-order effective Hamiltonian $H_{e}^{(1)}$ can be represented in real space using Pauli matrices and the unit matrix $\sigma_{o}$ as

$$
H_{e}^{(2)}=(-i \nabla-\mathbf{A}) \cdot \boldsymbol{\sigma}+V \sigma_{o}+\Delta \sigma_{z}
$$

The vector potential $\mathbf{A}$ in $H_{e}^{(2)}$ has the form of a magnetic field and is called the pseudomagnetic field.

\subsection{Perturbation theory for the effective Hamiltonian}

By using perturbation theory for the graphene first-order effective Hamiltonian $H_{e}^{(1)}$ when the lattice mismatch approaches zero, we can derive the band gap of the incommensurable system from that of the commensurable configurations. In this section we ignore the overlap matrix in Eq. 2.31 for simplicity and restrict ourselves to the neighbourhood of the $\mathbf{K}$ point. 
The two wavefunctions of pristine graphene are

$$
\langle\mathbf{r} \mid s, \mathbf{k}\rangle=\frac{e^{i \mathbf{k} \cdot \mathbf{r}}}{\sqrt{2}}\left(\begin{array}{c}
1 \\
i s e^{i \theta_{\mathbf{k}}}
\end{array}\right)
$$

where $s( \pm 1)$ is the chirality index of the states. The matrix elements of the potentials between the two states are

$$
\begin{aligned}
\left\langle s, \mathbf{k}\left|V \sigma_{o}\right| s^{\prime}, \mathbf{k}-\mathbf{g}\right\rangle & =\frac{1+s s^{\prime} e^{-i \theta_{\mathbf{k}, \mathbf{k}-\mathbf{g}}}}{2} V_{\mathbf{g}}, \\
\left\langle s, \mathbf{k}\left|\Delta \sigma_{z}\right| s^{\prime}, \mathbf{k}-\mathbf{g}\right\rangle & =\frac{1-s s^{\prime} e^{-i \theta_{\mathbf{k}, \mathbf{k}-\mathbf{g}}}}{2} \Delta_{\mathbf{g}},
\end{aligned}
$$

and

$$
\left\langle s, \mathbf{k}|\mathbf{A} \cdot \boldsymbol{\sigma}| s^{\prime}, \mathbf{k}-\mathbf{g}\right\rangle=i \frac{s^{\prime} e^{i \theta_{\mathbf{k}-\mathbf{g}} A_{-, \mathbf{g}}-s e^{-i \theta_{\mathbf{k}}} A_{+, \mathbf{g}}}}{2}
$$

where $A_{ \pm}=A_{x} \pm i A_{y}$. In first-order perturbation theory, the eigenvalues are the solutions of the secular equation

$$
\left|\left(\begin{array}{cc}
V_{0}+\mathfrak{I}\left(e^{-i \theta_{\mathbf{k}}} A_{+, 0}\right) & \Delta_{0}-i \mathfrak{R}\left(e^{-i \theta_{k}} A_{+, 0}\right) \\
\Delta_{0}+i \mathfrak{R}\left(e^{-i \theta_{k}} A_{+, 0}\right) & V_{0}-\mathfrak{I}\left(e^{-i \theta_{\mathbf{k}}} A_{+, 0}\right)
\end{array}\right)-\varepsilon\right|=0 .
$$

The modulating potential $V$ does not open a gap due to the chiral nature of graphene states near the Dirac point [73]. The $A_{0}$ can be simplified for supercells by using graphene bonding and anti-bonding wavefunctions $\left(\left|\psi_{+}\right\rangle,\left|\psi_{-}\right\rangle\right.$, the $| \pm, 0\rangle$ of Eq. 2.39)

$$
A_{0}=\sum_{\alpha} \frac{1}{\varepsilon_{S, \alpha}}\left\langle\psi_{+}\left|H_{h}\right| \phi_{S, \alpha}\right\rangle\left\langle\phi_{S, \alpha}\left|H_{h}^{\dagger}\right| \psi_{-}\right\rangle
$$

where $\left|\phi_{S, \alpha}\right\rangle$ is a substrate wavefunction and $\varepsilon_{S, \alpha}$ the corresponding energy. For infinitely large supercells the hopping interactions of the graphene A and B sublattices with the substrate will span the same area (inside the supercell). Therefore for every specific environment for an A-sublattice atom, there will be the same environment for a B-sublattice atom and we have

$$
\sum_{j \in \mathrm{S}}\left(\sum_{i \in \mathrm{A}} H_{\mathrm{h}, i j}-\sum_{i \in \mathrm{B}} H_{\mathrm{h}, i j}\right) \sim N_{\partial} \gamma
$$

where on the right hand side of the equation $\gamma$ is the magnitude of the average interlayer hopping and $N_{\partial}$ is the number of atoms close to the boundary of the supercell. The only term remaining at the $\mathbf{K}$ point includes the terms related to the interaction of carbon atoms with substrate atoms close to the boundary of the supercell. For very large supercells $N_{\mathrm{BN}} \simeq N_{\mathrm{C}}$ and there are then $N_{T} \simeq 4 N_{C}^{2}$ atoms in the supercell. Since only $N_{\partial} \simeq 8 N_{C}$ of them are close to the boundary of the supercell, by 
using Eq. 2.44 we have

$$
A_{0} \sim \frac{N_{\partial}^{2}}{N_{T}^{4}} \gamma^{2}\left(\frac{1}{\varepsilon_{\mathrm{B}}}+\frac{1}{\varepsilon_{\mathrm{N}}}\right) \sim \frac{\gamma^{2}}{N_{C}^{2}}\left(\frac{1}{\varepsilon_{\mathrm{B}}}+\frac{1}{\varepsilon_{\mathrm{N}}}\right)
$$

This argument shows the cancellation of $A_{0}$ for large supercells where $N_{C}$ becomes very large when the band gaps obtained from solving Eq. 2.43 will be $2\left|\Delta_{0}\right|$. With the same argument that leads to the cancellation of $A_{0}$, we conclude that in $\Delta_{0}$ only the sublattice symmetry-breaking potential remains. We can calculate $\Delta_{0}$ from commensurable configurations. We showed in section II that the on-site terms have a simple Fourier expansion; therefore for large supercells we find that $2 \Delta_{0}=V_{\mathrm{C}, 0}-V_{\mathrm{C}^{\prime}, 0}$ where $C$ refers to the A-sublattice and $C^{\prime}$ to the B-sublattice. Fourier expansion of the diagonal terms allows us to use the three most symmetric configurations (a), (b) and (c) to calculate $\Delta_{0}=\left(\Delta^{(\mathrm{a})}+\Delta^{(\mathrm{b})}+\Delta^{(\mathrm{c})}\right) / 3$.

The relation of the on-site terms to the gaps of the corresponding commensurable configurations are

$$
\begin{aligned}
& G^{(\mathrm{a})}=-2 \Delta^{(\mathrm{a})}-\frac{\gamma_{\mathrm{B}}^{2}}{\varepsilon_{\mathrm{B}}}+\frac{\gamma_{\mathrm{N}}^{2}}{\varepsilon_{\mathrm{N}}} \\
& G^{(\mathrm{b})}=2 \Delta^{(\mathrm{b})}+\frac{\gamma_{\mathrm{N}}^{2}}{\varepsilon_{\mathrm{N}}} \\
& G^{(\mathrm{c})}=2 \Delta^{(\mathrm{c})}-\frac{\gamma_{\mathrm{B}}^{2}}{\varepsilon_{\mathrm{B}}}
\end{aligned}
$$

where the $G$ are band gaps for commensurable configurations. This results in

$$
2\left|\Delta_{o}\right|=\frac{\left|G^{(\mathrm{a})}-G^{(\mathrm{b})}-G^{(\mathrm{c})}\right|}{3}
$$

This is the main result of perturbation theory for the effective Hamiltonian and predicts that the total gap of large supercells can be derived from the band gaps of just a few commensurable configurations. Using the LDA band gaps for the (a), (b) and (c) configurations, Eq. 2.48 predicts an LDA band gap of $5 \mathrm{meV}$ for the "wavy" $d_{\mathrm{eq}}^{\mathrm{RPA}}(\mathbf{r})$ structure and $31 \mathrm{meV}$ using the $G_{0} W_{0}$ corrected band gaps where the result of full diagonalization are 5 and 32 respectively. This close agreement suggest that our effective Hamiltonian captures the most important physics of the $\overline{\mathrm{C}} \mid h$-BN system. It is worth noting that if we neglect the interface dipole potential $V(\mathbf{r})$ and $\Delta(\mathbf{r})$ and only consider the hopping terms, the gap opened from Eq. 2.43 will be negligible. This means that a tight binding model that neglects the dipole potentials will not open a band gap.

We can derive the second order perturbation contribution to the effective Hamiltonian. The general form of the second-order energy corrections is

$$
\varepsilon_{s \mathbf{k}}^{(2)}=\sum_{s^{\prime}, \mathbf{g}} \frac{\left|\left\langle s, \mathbf{k}|\delta H| s^{\prime}, \mathbf{k}-\mathbf{g}\right\rangle\right|^{2}}{\varepsilon_{s \mathbf{k}}^{(0)}-\varepsilon_{s^{\prime}, \mathbf{k}-\mathbf{g}}^{(0)}}
$$


where $\delta H$ is the perturbing part of $H_{e}^{(2)}$ and the sum is over the states for which the denominator is not zero. The term which contains $\Delta$ is

$$
\varepsilon_{s k}^{(2) \Delta}=-2 s \sum_{\mathbf{g}} \frac{g \cos \theta_{k, g}}{g^{2}-2 k g \cos \theta_{k, g}}\left|\Delta_{\mathbf{g}}\right|^{2}
$$

Because of the global six-fold symmetry of the system, the above term and all of the other contributions cancel at the $\mathbf{K}$ point making the second-order correction to the band gap zero. This means that our perturbative estimate of the band gap in Eq. 2.48 is correct to second order.

\subsection{Screening}

Our TB analysis shows that placing graphene on a $h$-BN substrate leads to pristine graphene experiencing a perturbation that can be described as an external potential $V_{0}(\mathbf{r})$. This potential would in reality be screened. In DFT, the total potential could be expressed as the sum of an initial (bare) potential $V_{0}(\mathbf{r})$ and the potential $V_{\text {ind }}(\mathbf{r})$ induced by charge redistribution in the system $[74,75]$

$$
V_{\text {ind }}(\mathbf{r})=\frac{e^{2}}{\epsilon} \int d \mathbf{r}^{\prime} \frac{\delta n\left(\mathbf{r}^{\prime}\right)}{\left|\mathbf{r}-\mathbf{r}^{\prime}\right|}+V_{x c}(\mathbf{r})
$$

where $V_{x c}$ is the exchange-correlation potential of the system. $V_{x c}$ can be neglected in comparison with the first term, the Hartree potential. The electron density of the system $n(\mathbf{r})$ can be determined by using the density of states (DoS), which itself can be related to the chemical potential $\mu$ by

$$
n(\mu)=\frac{\mu|\mu|}{\pi \hbar^{2} v_{F}^{2}}
$$

For a free-standing graphene layer in an external potential $V_{0}(\mathbf{r})$, the total potential can be explicitly written as

$$
\begin{aligned}
V(\mathbf{r})=V_{0}(\mathbf{r})+ & \frac{e^{2}}{\pi \kappa \hbar^{2} v_{F}^{2}} \int d \mathbf{r}^{\prime} \mu \frac{\left|\mu-V\left(\mathbf{r}^{\prime}\right)\right|-|\mu|}{\left|\mathbf{r}-\mathbf{r}^{\prime}\right|} \\
& -\frac{e^{2}}{\pi \kappa \hbar^{2} v_{F}^{2}} \int d \mathbf{r}^{\prime} V\left(\mathbf{r}^{\prime}\right) \frac{\left|\mu-V\left(\mathbf{r}^{\prime}\right)\right|}{\left|\mathbf{r}-\mathbf{r}^{\prime}\right|}
\end{aligned}
$$

where $\kappa$ is the relative dielectric constant of the environment including vacuum on one side of the graphene layer and $h$-BN on the other side, $\kappa=(1+2.8) / 2$ [61]. This is the general Thomas-Fermi (TF) equation for graphene. In such a system, the TF model can describe static charge redistributions where it is equivalent to the zerofrequency, long wavelength $(q=0, \omega=0)$ limit of the RPA model. Therefore if the system does not have a global gap, there will be charge redistribution. If there is a gap, no static $(\omega=0)$ transition can change the charge distribution and the TF model 
fails to describe screening in such a system. We should instead use a frequencydependent dielectric function such as the RPA. From section IV we already know that the band gap is zero for $\overline{\mathrm{C}} \mid h$-BN configurations with large misalignments making the TF model applicable to these configurations.

\subsubsection{Doped graphene}

We begin by examining screening in doped graphene. Because Eq. 2.53 depends on absolute values, we consider a free-standing graphene layer doped to the maximum of its bare potential $\left(\mu=\left|V_{0}(\mathbf{r})\right|_{\max }\right)$. This choice allows us to simplify one of the absolute values and therefore, write its TF equation as

$$
V(\mathbf{r})=V_{0}(\mathbf{r})+\frac{e^{2}}{\pi \kappa \hbar^{2} v_{\mathrm{F}}^{2}} \int d \mathbf{r}^{\prime} V\left(\mathbf{r}^{\prime}\right) \frac{V\left(\mathbf{r}^{\prime}\right)-2|\mu|}{\left|\mathbf{r}-\mathbf{r}^{\prime}\right|}
$$

The Fourier transform of this equation will lead to

$$
V_{\mathbf{g}}=V_{0, \mathbf{g}}+\frac{2 r_{s}}{\hbar v_{\mathrm{F}}|g|} \sum_{\mathbf{q}} V_{\mathbf{q}}\left(V_{\mathbf{g}-\mathbf{q}}-2|\mu| \delta_{\mathbf{q}, \mathbf{g}}\right) .
$$

An approximate solution to this equation when $V_{0, \mathrm{~g}}$ is small can be obtained as

$$
V_{\mathbf{g}}=\frac{V_{0, \mathbf{g}}}{1+q_{\mathrm{TF}} / g}
$$

where $q_{\mathrm{TF}}$ is the characteristic wavevector in the TF model

$$
q_{\mathrm{TF}}=\frac{4 r_{s}|\mu|}{\hbar v_{\mathrm{F}}}=\frac{2 \pi}{L_{\mathrm{TF}}}
$$

For large supercells, the results of the TF model agree with the RPA result[76]. The exact solution when the initial modulating potential $\left(V_{0}\right)$ contains only the six smallest reciprocal lattice vectors in its Fourier expansion is

$$
V_{\mathrm{g}}=\frac{|\mu|}{2} \frac{g}{q_{\mathrm{TF}}}\left[1+\frac{q_{\mathrm{TF}}}{g}-\sqrt{\left(1+\frac{q_{\mathrm{TF}}}{g}\right)^{2}-\frac{4 V_{0, \mathrm{~g}}}{|\mu|} \frac{q_{\mathrm{TF}}}{g}}\right]
$$

The results of Eq. 2.56 and Eq. 2.58 are shown in Fig. 2.11 for the $V_{0, g}$ values derived from many-body calculations. The Thomas-Fermi length is $L_{\mathrm{TF}} \sim 6.3 \mathrm{~nm}$.

\subsubsection{Undoped graphene}

If the graphene layer is doped less than the modulating potential, the TF model becomes sensitive to the shape of the bare potential. Here we study undoped graphene 


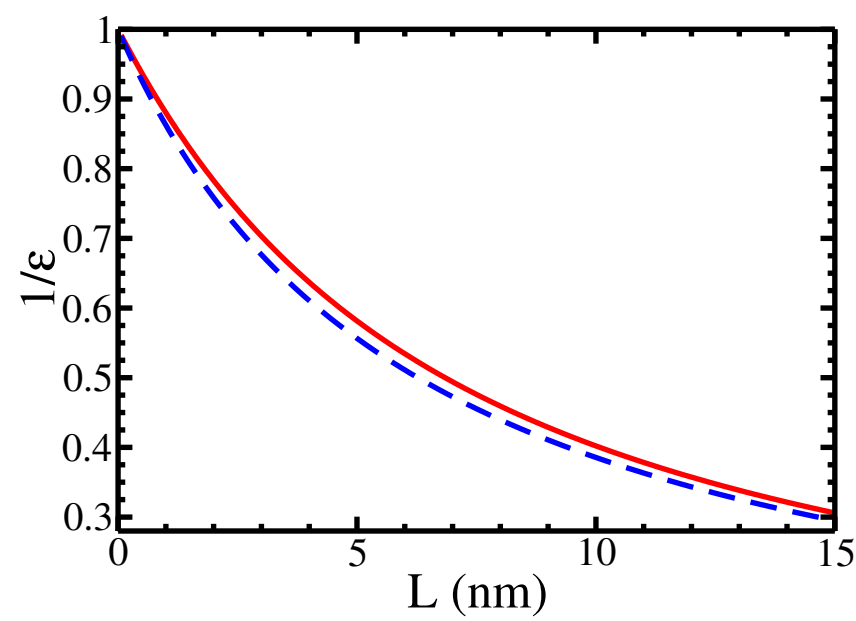

Figure 2.11: TF results for doped graphene, Eq. 2.56 (blue dashed line) and Eq. 2.58 (red).

as a limiting case. For undoped graphene the TF equation becomes:

$$
V_{\mathbf{g}}=V_{0, \mathbf{g}}-\frac{2 r_{s}}{\hbar v_{\mathrm{F}} g} \int_{\Omega} \frac{d \mathbf{r}}{\Omega} V(\mathbf{r})|V(\mathbf{r})| e^{-i \mathbf{g} \cdot \mathbf{r}}
$$

where $\Omega$ is the area of the supercell and the integral is over the supercell. The above integral can be separated into two integrals as follows:

$$
\frac{1}{\Omega}\left(\int_{\Omega^{+}} d \mathbf{r} V^{2}(\mathbf{r}) e^{-i \mathbf{g} . \mathbf{r}}-\int_{\Omega^{-}} d \mathbf{r} V^{2}(\mathbf{r}) e^{-i \mathbf{g} \cdot \mathbf{r}}\right)
$$

where $\Omega^{+}\left(\Omega^{-}\right)$are the regions where $V$ is positive (negative). The boundaries of these two integration regions depend on the shape of the screened potential, and should be found from Eq. 2.59 simultaneously with the potential itself. We can assume that the area of these regions will not be changed by screening for the average of the periodic potential over the supercell is not changed by screening in the TF model; screening only changes the magnitude of the potential and not its phase. We could choose the supercell in such a way that the $\Omega^{+}$region is in its center, but by doing so the $\Omega^{-}$region would have a complicated shape inside the supercell. If we choose the external potential to have a simple shape so that its Fourier expansion contains only the six smallest reciprocal lattice vectors, all with the same phase and magnitude, then we can show that the $\Omega^{+}$region is a simply-connected hexagonal while the other region has a more complicated shape. If we start with the positive $V_{0, \mathbf{g}}$, then the positive part $\left(\Omega^{+}\right)$is hexagonal; we approximate this hexagon with a circle of the same area since the details of the boundary do not change the general trend of the screening. We define $\tau=\Omega^{+} / \Omega$ as the fraction of the supercell where the 
potential is repulsive. This assumption leads to an analytical solution for the integral in Eq. 2.60

$$
\int_{\Omega^{+}} \frac{d \mathbf{r}}{\Omega} V^{2}(\mathbf{r}) e^{-i \mathbf{g} \cdot \mathbf{r}}=2 \tau \sum_{\mathbf{p}, \mathbf{q}} \frac{J_{1}(|\mathbf{p}+\mathbf{q}+\mathbf{g}| R)}{|\mathbf{p}+\mathbf{q}+\mathbf{g}| R} V_{\mathbf{p}} V_{\mathbf{q}}
$$

where $R=\left(\frac{\tau \Omega}{\pi}\right)^{1 / 2}$ is the radius of the corresponding circle and $J_{1}$ is the Bessel function of the first kind. The oscillatory function $J_{1}(r) / r$ originates from the non-linear dependence of the TF model on the absolute value of $V$. The oscillations of $J_{1}(r) / r$ make the screened potential a non-monotonic function of the reciprocal lattice vectors g. Eq. 2.60 then simplifies to

$$
\int_{\Omega} \frac{d \mathbf{r}}{\Omega} V|V| e^{-i \mathbf{g} \cdot \mathbf{r}}=2 \int_{\Omega^{+}} \frac{d \mathbf{r}}{\Omega} V^{2} e^{-i \mathbf{g} \cdot \mathbf{r}}-\int_{\Omega} \frac{d \mathbf{r}}{\Omega} V^{2} e^{-i \mathbf{g} \cdot \mathbf{r}}
$$

and collecting these parts leads to

$$
V_{\mathbf{g}}=V_{0, \mathbf{g}}-\frac{2 r_{s}}{\hbar v_{F} g} \sum_{\mathbf{p}, \mathbf{q}} V_{\mathbf{p}} V_{\mathbf{q}}\left(\frac{4 \tau J_{1}(|\mathbf{p}+\mathbf{q}+\mathbf{g}| R)}{|\mathbf{p}+\mathbf{q}+\mathbf{g}| R}-1\right)
$$

If we take the magnitude of the smallest reciprocal lattice vector for the supercell to be $G$, then we can define $\alpha \equiv G R=\left(12 \tau^{2} \pi^{2}\right)^{1 / 4}$, and using our assumption that only the magnitude of $V_{\mathbf{g}}$ is changed by screening, then for every $\mathrm{g}$ we can group the $\mathbf{p s}$ and $\mathbf{q s}$ into sets where $|\mathbf{p}+\mathbf{q}+\mathbf{g}|$ are the same. For example, we define the first set to be $\{1\}_{\mathbf{g}} \equiv[\mathbf{p}, \mathbf{q} \ni|\mathbf{p}+\mathbf{q}+\mathbf{g}|=G]$, the second set to be $\{2\}_{\mathbf{g}} \equiv$ $[\mathbf{p}, \mathbf{q} \ni|\mathbf{p}+\mathbf{q}+\mathbf{g}|=\sqrt{3} G]$, etc. For each set, the value of the quantity between the large brackets in Eq. 2.63 is constant for all ps and qs belonging to the set and related to $\alpha$. If we define this value to be $\beta$ then for the first four groups we have $\beta=$ $-0.45,-0.95,-1.06,-1.12$. Solving Eq. 2.63 is still difficult so we only solve it for $g=G$ and the first set group and only for the ps and qs whose magnitude is $\mathbf{G}$. There are two pairs of $\mathbf{p}$ and $\mathbf{q}$ for which $|\mathbf{p}+\mathbf{q}+\mathbf{g}|=0$, but since $\lim _{x \rightarrow 0} 2 J_{1}(x) / x-1=0$ then they do not enter into the equation. There are 15 pairs of $\mathbf{p}$ and $\mathbf{q}$ for which $|\mathbf{p}+\mathbf{q}+\mathbf{G}|=G$ and therefore we have

$$
V_{g}=V_{0, g}-\frac{30 \beta r_{s}}{\hbar v_{F} g} V_{g}^{2}
$$

where $\beta=-0.45$ and thus $V_{g}$ can be solved

$$
V_{g}=\frac{\hbar v_{F} g}{60 \beta r_{s}}\left(1-\sqrt{1-120 \beta r_{s} \frac{V_{0, g}}{\hbar v_{F} g}}\right) .
$$

For small supercells we can write

$$
V_{g} \sim \frac{V_{0, g}}{1-30 \beta r_{s} \frac{V_{0, g}}{\hbar v_{F} g}}
$$




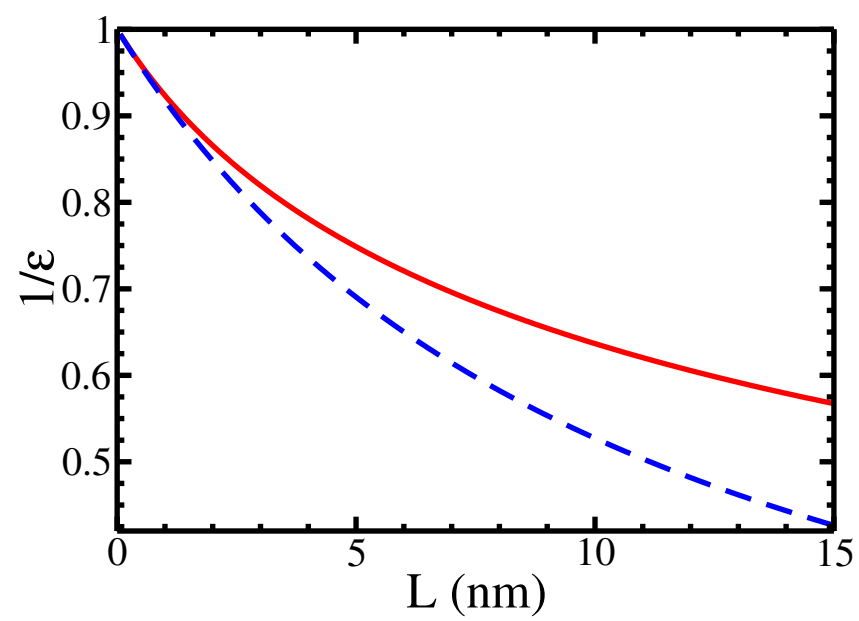

Figure 2.12: TF results for undoped graphene. Results from Eq. 2.65 (solid red) and Eq. 2.66 (dashed blue).

which gives us a potential dependent TF wavevector. For typical values that we found for $\overline{\mathrm{C}} \mid h$-BN we know that the variation in the modulating potential of quasiparticles is about $235 \mathrm{meV}$, and therefore $V_{0, g} \sim 235 / 9=26 \mathrm{meV}$ and $r_{s} \sim 1.14$ and $\hbar v_{F} g=2 \pi \gamma \frac{g}{b}$ where $b$ is the primitive reciprocal lattice vectors of graphene and $\gamma$ is the nearest neighbour hopping in graphene, therefore the TF length scale will be $L_{\mathrm{TF}} \sim 11.2 \mathrm{~nm}$, and the dielectric function is $\epsilon=1-30 \beta r_{s} V_{0, g} / \hbar v_{F} g$.

There is no characteristic TF wavevector corresponding to Eq. 2.65 because the system does not have any characteristic length scale when the graphene layer is undoped. However, we can still compare the doped and undoped systems, the $V$ behaviour changes from a $g$ dependence for doped graphene to a $g^{1 / 2}$ for an undoped system in very large supercells. In the RPA approximation, the static polarization function of graphene[76] when $\hbar v_{F} g \geq 2|\mu|$ is

$$
\Pi_{0}(g)=\frac{2|\mu|}{\pi \hbar v_{\mathrm{F}}}\left[1-\frac{1}{2} \sqrt{1-\left(\frac{2|\mu|}{\hbar v_{\mathrm{F}} g}\right)^{2}}-\frac{\hbar v_{\mathrm{F}} g}{4|\mu|} \sin ^{-1} \frac{2|\mu|}{\hbar v_{\mathrm{F}} g}\right]
$$

and for $\hbar v_{\mathrm{F}} g \leq 2|\mu|$ we have

$$
\Pi_{0}(g)=\frac{2|\mu|}{\pi \hbar^{2} v_{\mathrm{F}}^{2}}\left[1-\frac{\pi}{4} \frac{\hbar v_{\mathrm{F}} g}{2|\mu|}\right]
$$

In the long wavelength limit for a doped system, the screening wavevector of the dielectric function in the RPA approaches the corresponding wavevector of TF di- 
electric function

$$
q_{s}=\frac{2 \pi e^{2}}{\kappa} \Pi_{0}(0)=\frac{4 r_{s}|\mu|}{\hbar v_{\mathrm{F}}}=q_{\mathrm{TF}}
$$

For undoped free-standing graphene with no gap, there is no screening length scale, but an effective dielectric constant can be derived from Eq. 2.14 and Eq. 2.67

$$
\epsilon=1+\frac{\pi r_{s}}{2}
$$

This dielectric function gives $1 / \epsilon \sim 0.36$ which is smaller than the values from Fig. 2.8 and is closer to the results for doped systems. Comparison of Fig. 2.11 and Fig. 2.12 shows that doped graphene can screen the induced modulating potential more strongly than undoped graphene.

\subsection{Conclusions}

Many-body corrections to a tight binding model developed here for the $\overline{\mathrm{C}} \mid h$-BN system show that the quasiparticle energies of this system around the $\mathbf{K}$ point are much larger than was previously thought. Our relaxation of the interface of the $\overline{\mathrm{C}} \mid h$-BN system leads to a wavy topography for the graphene layer, which along with the many-body corrections results in a gap of about $30 \mathrm{meV}$. This band gap is the result of an incomplete cancellation of the sublattice symmetry-breaking potential for the graphene bands near the $\mathbf{K}$ point, suggesting that an amplified height variation of the graphene layer might allow us to make use of the underlying large gaps to open a wider gap in the graphene layer; this can, for example, be done through locally bending the surface at some regions of the supercells. Our final tight binding picture can be employed to understand the physics behind the $\overline{\mathrm{C}} \mid h-\mathrm{BN}$ interlayer interactions.

We developed a perturbation scheme to predict the band gaps of large supercells using small commensurable configurations. We found that an effective Hamiltonian assigned to the $\overline{\mathrm{C}} \mid h$-BN system suffices to understand the interactions of the system, and can be even be applied to more complex situations where external pressure is applied. Furthermore, the screening of the potentials in the $\overline{\mathrm{C}} \mid h$-BN system can be understood from our RPA dielectric function derived for the gapped-graphene; this screening can also be applied to other systems where a band gap is opened for graphene. Employing the Thomas-Fermi model, we have demonstrated that the behaviour of the $\overline{\mathrm{C}} \mid h$-BN system changed as a function of the size of the supercell, and consequently, the metal-insulator transition in the $\overline{\mathrm{C}} \mid h$-BN system changes the screening of the system. It is also noteworthy that our theoretical model developed here for the $\overline{\mathrm{C}} \mid h$-BN system can be applied to any other substrate-supported graphene layer, too. 


\section{3}

\section{Intrinsic electron-hole puddles in}

graphene on hexagonal boron nitride

\subsection{Introduction}

Hexagonal boron nitride ( $h$-BN) has been shown to be a very suitable dielectric substrate for graphene devices. It has insulating characteristics similar to those of $\mathrm{SiO}_{2}$ [77] but has far fewer charged impurities and is atomically flat. This results in a higher mobility of the charge carriers in graphene when placed on $h$-BN compared to $\mathrm{SiO}_{2}$ [42] and larger electron-hole puddles [72] which are less deep [43, 44]. Graphene has a small lattice mismatch with $h$-BN and forms a superstructure when placed on it which is observed as a moiré pattern in scanning tunnelling microscopy (STM) images [43, 44]. Since STM does not directly measure the height but rather a current, it is possible that the moiré patterns in graphene $(\overline{\mathrm{C}})$ on $h$-BN $(\overline{\mathrm{C}} \mid h-\mathrm{BN})$ have an electronic as well as a structural origin. If the local density of states, i.e. the doping level, gradually fluctuates throughout the graphene sheet, the STM tip will follow this profile in order to keep the tunnelling current constant.

The $h$-BN honeycomb crystal structure has an approximately $\delta=1.8 \%$ larger lattice constant than graphene. Unlike graphene, the bonds in the $h$-BN plane have a polar-covalent character because of the large difference in electronegativity of boron and nitrogen. Graphene is weakly bonded to $h$-BN with a binding distance similar to the $h$-BN interlayer separation [18, 45]. The competition between in-plane strain and interplanar bonding in $\overline{\mathrm{C}} \mid h$-BN has been investigated by means of random phase approximation (RPA) calculations within the framework of the adiabatic connection fluctuation-dissipation theorem (ACFDT) calculations [45]. There it was found that the energy cost of stretching the graphene sheet and compressing $h$-BN to make them match was comparable in size to the gain in bonding energy making it uncer- 


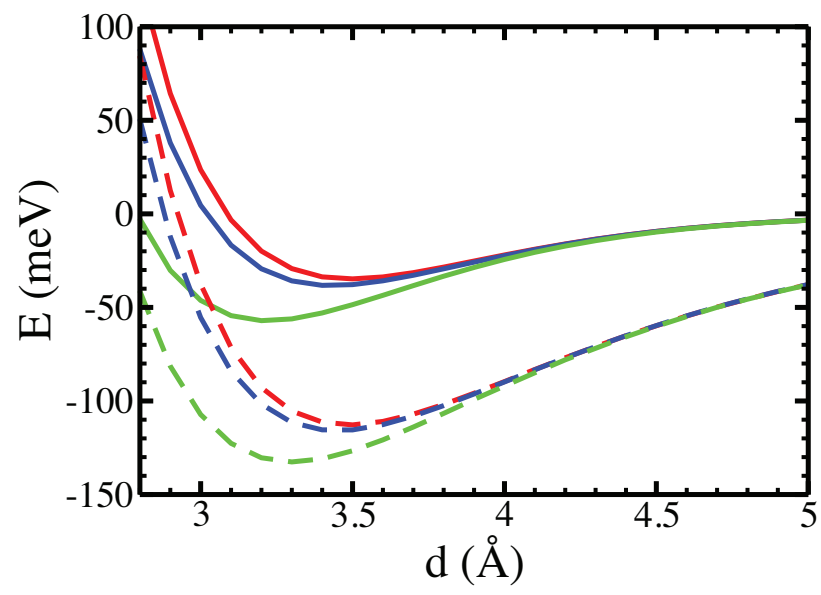

Figure 3.1: Binding energy curves for commensurable aligned $\overline{\mathrm{C}} \mid h-\mathrm{BN}$ in the three symmetrical configurations (a) red, (b) blue and (c) green explained in the text. The dashed lines correspond to vdW-DF, the solid lines to LDA calculations.

tain what would actually happen in experiment. Subsequent experiments indicated that when very well aligned, graphene and $h$-BN form domains where they become commensurable that are separated by domain walls where the strain accumulates [56]. In the aligned case, we have predicted that band gaps of order $30 \mathrm{meV}$ would develop for the incommensurable case and of order $200 \mathrm{meV}$ if the lattices remain incommensurable [52]. Experimentally, temperature dependent measurements indicate gaps of $\sim 30 \mathrm{meV}[48,56]$. Above a very small critical angle, the lattices no longer become commensurable. This is the case that is studied in this chapter.

\subsection{Computational details}

The DFT equilibrium binding distances and charge densities for different $\overline{\mathrm{C}} \mid h$-BN configurations were calculated using the Local Density Approximation (LDA) [3] and vdW-DF [78] functionals within the framework of the plane-wave PAW pseudopotential method [8], as implemented in VASP [59, 79]. A plane wave basis with a cutoff energy of $600 \mathrm{eV}$ in combination with a $36 \times 36 \times 1$ k-point grid was used. Total energies were calculated using the tetrahedron scheme [80] and converged to $10^{-7}$ $\mathrm{eV}$. Care was taken to ensure that the high symmetry $\boldsymbol{\Gamma}, \mathbf{K}$, and $\mathbf{M}$ points of graphene were explicitly included in the Brillouin zone sampling in order to describe charges and band structures accurately. A dipole correction was applied to avoid spurious interactions between periodic images of the slab [81].

For the rotationally aligned commensurable structures a unit cell with $a_{\text {hex }}=$ $2.445 \AA$ consists of two carbon atoms on top of a $h$-BN and a $15 \AA$ vacuum layer. For the large rotated $\overline{\mathrm{C}} \mid h$-BN structures a k-grid with the same density was used. 


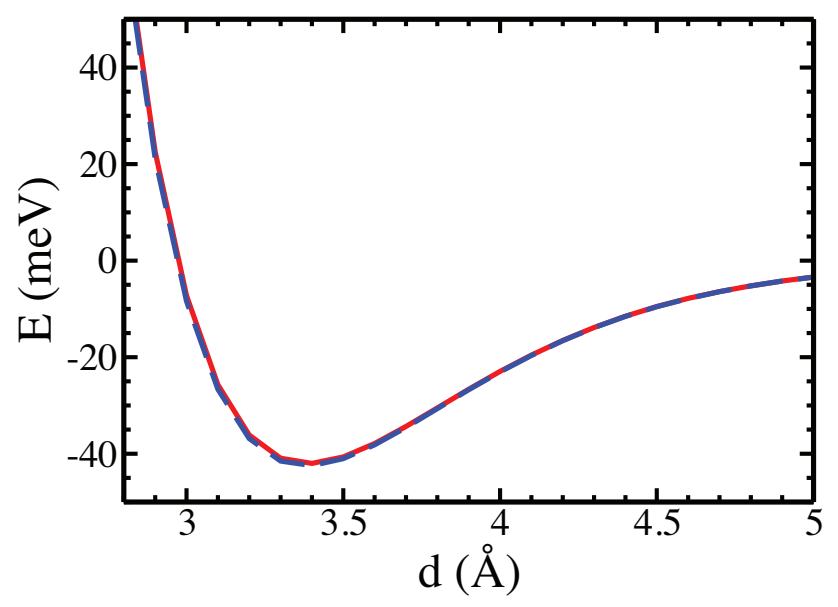

Figure 3.2: LDA Binding energy curves for two commensurable supercells with the graphene and $h$-BN lattice rotated by $\theta=21.8^{\circ}$ (solid red) and $\theta=11.0^{\circ}$ (dashed blue) with respect to one another.

\subsection{Graphene $\mid h$-BN binding}

In Fig. 3.1 the binding energies of three commensurable aligned $\overline{\mathrm{C}} \mid h$-BN structures are shown as a function of the binding distances $d$. We distinguish three different symmetrical configurations: (a) with one carbon over boron, the other over nitrogen; (b) with one carbon over nitrogen, the other centered above a $h$-BN hexagon; (c) with one carbon over boron, the other centred above a $h$-BN hexagon. The LDA curve decays exponentially, since it depends on the wavefunction overlap between the two sheets, whereas the vdW-DF curves show a $d^{-4}$ dependence, as expected for the asymptotic behaviour for van der Waals interacting planes [82]. As expected, the vdW-DF yields larger equilibrium binding separations. A comparison with the results from the ACFDT-RPA method shows that the energy ordering of the three configurations does not depend on which functional is used. The equilibrium separations are largely similar, with the exception of the (c) configuration where the LDA results in a significantly shorter separation than either vdW-DF or RPA.

We also calculated the binding energy curves for two commensurable systems rotated through $\theta=21.8^{\circ}$ ( 7 unit cells of graphene) and $\theta=11.0^{\circ}$ (13 unit cells of graphene). The LDA binding energy curves shown in Fig. 3.2 are almost identical with the same equilibrium binding separation. The difference between the equilibrium binding energies is much less than what is seen for the aligned systems in Fig. 3.1 indicating that for the rotated systems the binding energy will not depend on the detailed structure of the supercell.

Even though the interaction between graphene and $h$-BN is small, a non-negligible 


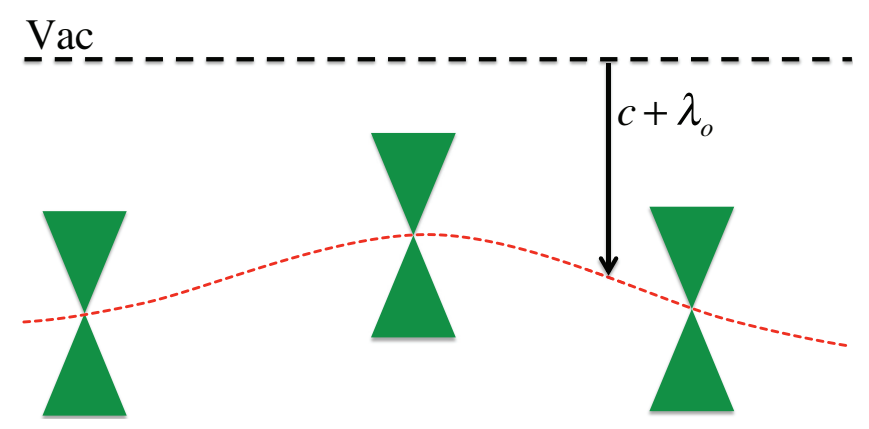

Figure 3.3: Schematic illustration of the local potential and local Dirac cones that follow the potential. $c$ is a constant potential and Vac indicates the vacuum level of the system.

interface dipole layer is formed at the interface [60]. The strength and direction of the dipole depend on the binding configuration. The interface dipole is calculated from the charge density difference function $\Delta n(\mathbf{x})=n_{\overline{\mathrm{C} \mid \mathrm{BN}}}(\mathbf{x})-n_{\overline{\mathrm{C}}}(\mathbf{x})-n_{\mathrm{BN}}(\mathbf{x})$. Formation of the dipole layer gives rise to a step $\Delta V$ in the electrostatic potential perpendicular to the layer as

$$
\Delta V=\frac{e^{2}}{\Omega \varepsilon_{o}} \int_{-\infty}^{\infty} z d z \int_{\Omega} d x d y \Delta n(\mathbf{x})
$$

where $\Omega$ is the area of the supercell, $x, y$ are in-plane coordinates and $z$ is the out of plane coordinate. We found that the results of vdW-DF and LDA are very close to each other and therefore $\Delta V$ could be taken as a functional-independent parameter [57]. This results indicates that the rather crude LDA functional gives a very reasonable description of potentials in this weakly van der Waals bonded system at lower computational cost. In the remainder of this work we will therefore use RPA equilibrium separations [45] but use the LDA to carry out calculations.

\subsection{Puddle formation}

With both lattice mismatch and orientational misalignment there is in principle no limit to the moiré pattern period for $\overline{\mathrm{C}} \mid h$-BN depending on the rotation angle. The resulting interface dipole will therefore be slowly varying throughout the supercell for large moiré patterns. This slow variation enables us to take the local electronic structure as similar to the Dirac cone structure of graphene by a local Hamiltonian. A schematic illustration of the consequence of this is shown in Fig. 3.3. Locally the potential step $\left(\lambda_{o}\right)$ changes, creating a landscape of shifted Dirac cones. The graphene sheet will try to screen the external potential, however because of the low density of states near the Fermi energy, it is not able to completely do so. The strength of the potential will be diminished, but the shape is retained. Therefore we believe that even 


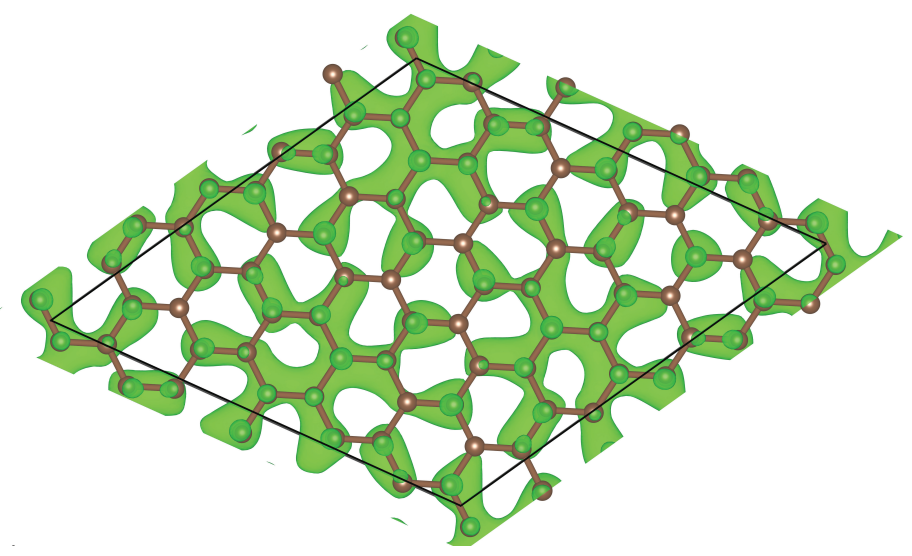

Figure 3.4: Charge density difference of $\overline{\mathrm{C}} \mid h$-BN around the graphene sheet for a supercell with $\theta=17.9^{\circ}$ containing 62 carbon atoms.

with screening in the graphene sheet, the effect of the local binding configuration on the interface dipole will give rise to lateral charge redistribution by the screened potential. This will become apparent by electron and hole doped regions in a graphene sheet on $h$-BN. In the case that a band gap opens, but the size of band gap is smaller than the $\lambda_{o}$, then still an in-plane charge redistribution will occur, and in the case that the band gap is larger than the potential step and graphene in its undoped state, electron-hole puddles can not formed.

\subsubsection{Low energy Hamiltonian}

The low energy Hamiltonian of graphene will contain the two bands around the $\mathbf{K}$ point. The general form of this Hamiltonian in real space can be represented as

$$
H_{\mathbf{K}}(\mathbf{x})=\lambda_{o} \sigma_{o}+\lambda_{m} \sigma_{z}+\left(-i \hbar v_{F} \nabla+\mathbf{f}\right) \cdot \boldsymbol{\sigma}
$$

where $\mathbf{x}$ is the position of the carbon pairs measured in the supercell, $v_{F}$ is the Fermi velocity, $\sigma$ represents the sublattice degree of freedom. $\lambda_{o}$ is the modulating potential which shifts the position of the graphene's bands in energy, $\lambda_{m}$ is the sublattice symmetry breaking term which can rises from dipole layer formation and interlayer hoppings and can correspond to the breaking of the inversion symmetry in the system, and $\mathbf{f}$ is a two-dimensional vector that represent breaking of the $C_{3}$ threefold symmetry in the system. In the supercell the Hamiltonian in Eq. 3.2 will be position-dependent and therefore the parameters will be function local of position of graphene's original primitive unitcell in the supercell. For large supercells the parameters of Hamiltonian will depend on the position of graphene's primitive cell.

The slowly varying landscape means that the Hamiltonian. Eq. 3.2 can be locally diagonalized and local band structure and wavefunctions can be calculated. If we 
define the average of our parameters around some region in the supercell $(\overline{\mathbf{x}})$ then Hamiltonian can be partitioned $(H(\overline{\mathbf{x}}))$ and therefore local energies will be

$$
\varepsilon_{ \pm}(\overline{\mathbf{x}})=\lambda_{o}(\overline{\mathbf{x}}) \pm \sqrt{\lambda_{m}^{2}(\overline{\mathbf{x}})+\mathbf{f}^{2}(\overline{\mathbf{x}})}
$$

and the local wave functions are

$$
\psi_{ \pm}(\overline{\mathbf{x}})=e^{i \varphi(\overline{\mathbf{x}})}\left(\lambda_{m}(\overline{\mathbf{x}}) \pm \sqrt{\lambda_{m}^{2}(\overline{\mathbf{x}})+\mathbf{f}^{2}(\overline{\mathbf{x}})}, f(\overline{\mathbf{x}}) e^{i \theta_{f}}\right) / Z_{ \pm}
$$

where $Z_{ \pm}$is the normalization factor and $\theta_{f}$ is the angle of vector $\mathbf{f}$ and $\varphi$ is the position-dependent phase of the local wave function. Since the local wavefunctions should be continuously glued together in order to make the global wavefunction, then the phase of the local wave functions cannot be chosen arbitrarily. As we show in Eq. 3.4 the local wave functions do not depend explicitly on $\lambda_{o}$, but the continuity of the global wave function will requires indirectly introduce dependency on the $\lambda_{o}$ through $\varphi$. The normalization factor $Z_{ \pm}$does not depend on the local parameters but only normalizes the total wave function, and therefore the local wave functions will capture charge fluctuations through the supercell by being non-normalized. Locally the $f$ terms cannot be ignored since without them the wave function will not be continuous through the Brillouin zone, but if we partition the supercell into large enough cells with approximate three-fold symmetry in them, then the contribution of the $f$ term could be neglected.

\subsubsection{Global solutions}

For the general case the Hamiltonian Eq. (3.2) can be represented in reciprocal space and the Dirac equation for graphene becomes

$$
\sum_{\mathbf{g}_{i}}\left\langle s \mathbf{q}|\delta H| s^{\prime}, \mathbf{q}+\mathbf{g}_{i}\right\rangle\left\langle s^{\prime}, \mathbf{q}+\mathbf{g}_{i} \mid \psi_{s \mathbf{q}}\right\rangle=\left(\varepsilon_{s \mathbf{q}}-\varepsilon_{s \mathbf{q}}^{(o)}\right)\left\langle s \mathbf{q} \mid \psi_{s \mathbf{q}}\right\rangle
$$

where $\delta H=\lambda_{o} \sigma_{o}+\lambda_{m} \sigma_{z}+\mathbf{f} . \boldsymbol{\sigma}$ is the perturbative part of Hamiltonian, $\langle\mathbf{x} \mid s, \mathbf{q}\rangle=$ $e^{i \mathbf{q} \cdot \mathbf{x}}\left(1, s e^{i \theta_{q}}\right) / \sqrt{2}$ are the unperturbed wave functions of graphene and $s$ is the chirality index of the bands. If we choose the local regions large enough then $f$ will vanish from the Hamiltonian and therefore we have

$$
\left\langle s \mathbf{q}|\delta H| s^{\prime}, \mathbf{q}+\mathbf{g}\right\rangle=\frac{1}{2}\left[\lambda_{o}^{\mathbf{g}}+\lambda_{m}^{\mathbf{g}}+s s^{\prime}\left(\lambda_{o}^{\mathbf{g}}-\lambda_{m}^{\mathbf{g}}\right) e^{i\left(\theta_{\mathbf{q}+\mathbf{g}}-\theta_{\mathbf{q}}\right)}\right]
$$

where $\lambda_{o}^{\mathrm{g}}$ is the corresponding Fourier transformation of $\lambda_{o}$. Therefore the wave function can be written to first order as

$$
\left\langle\mathbf{x} \mid \psi_{s \mathbf{q}}\right\rangle=\langle\mathbf{x} \mid s \mathbf{q}\rangle+\sum_{i} \frac{\left\langle\mathbf{x} \mid s^{\prime}, \mathbf{q}+\mathbf{g}_{i}\right\rangle\left\langle s^{\prime}, \mathbf{q}+\mathbf{g}_{i}|\delta H| s \mathbf{q}\right\rangle}{\varepsilon_{s \mathbf{q}}^{(o)}-\varepsilon_{s^{\prime}, \mathbf{q}+\mathbf{g}_{i}}^{(o)}}
$$

this solution however works when $\lambda_{o}^{(g)}, \lambda_{m}^{(g)} \ll \hbar v_{F} q$ where $g$ is the smallest recipro- 


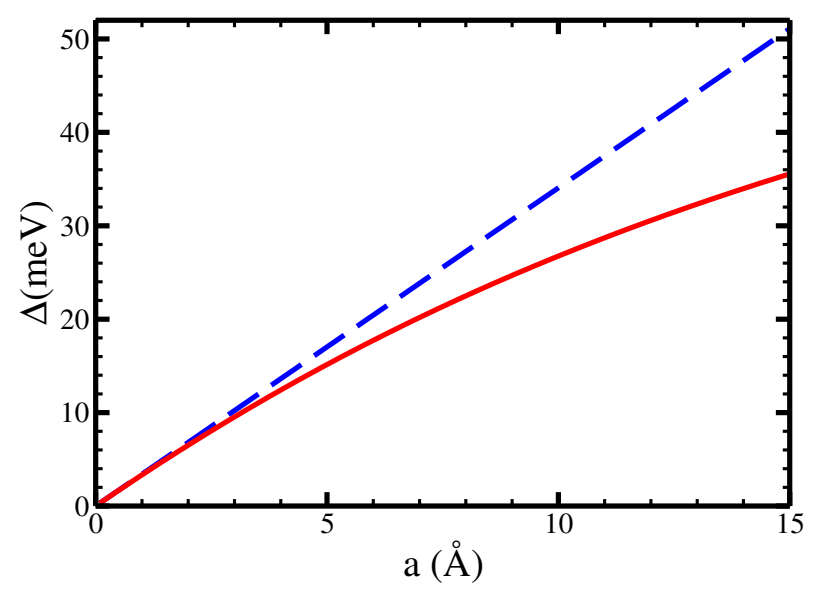

Figure 3.5: Energy gap from Eq. 3.8 where blue line contains up to the linear term and red line contains all terms.

cal lattice vector of the supercell, since it is a perturbation result for bands away from the gap. For bands very close to the gap then we need to use Eq. 3.5 and it is more convenient to change our basis to $\langle x \mid+, q\rangle=e^{i q \cdot x}(1,0)$ and $\langle x \mid-, q\rangle=e^{i q \cdot x}(0,1)$. We can look at the occupied wave function at the $\mathbf{K}$ point by solving Eq. 3.5 by using new basis. If we define $\varepsilon_{g}=\hbar v_{F} g$ as the lattice energy, where $g$ is the smallest reciprocal lattice vector and if we define $g=b / N$ where $b$ is the reciprocal lattice vector of graphene and $N$ is the scale of supercell to primitive unit cell, then we have $\varepsilon_{g}=2 \pi \gamma / N$ where $\gamma$ is the nearest neighbour hopping parameter of graphene, then the energy gap for the cases where

$$
\Delta=2 \lambda_{m}^{(o)}-\frac{12 \lambda_{o}^{(g)} \lambda_{m}^{(g)}}{\pi \gamma} N+\frac{6 \lambda_{m}^{(g)}\left[\left(\lambda_{m}^{(g)}\right)^{2}+3\left(\lambda_{o}^{(g)}\right)^{2}\right]}{\pi^{2} \gamma^{2}} N^{2}
$$

the last term in Eq. 3.8 is the higher order interactions and therefore if the last term is important, then larger $g$ vectors should be included which means that the wave functions and energies will no longer follow the potential. The gap at $a=14.5 \mathrm{~nm}$ is around $36 \mathrm{meV}$, which is in agreement with our previous findings [52]. We plot the linear term in Eq. 3.8 and the full energy gap in Fig. 3.5, and we show the potential and wavefunction difference

$$
\delta|\psi(\mathbf{x})|^{2}=|\psi(\mathbf{x})|^{2}-\left|\psi_{o}(\mathbf{x})\right|^{2}
$$

where $\psi_{o}(\mathbf{x})$ is the unperturbed wavefunction of graphene at the $\mathbf{K}$ point for the supercell of $55 \times 55$ graphene unit cell in Fig. 3.6. We used the corresponding values obtained from our commensurable DFT calculations with including many-body corrections for $\lambda_{o}$ as $235 \mathrm{meV}$ variation and $\lambda_{m}$ for $200 \mathrm{meV}$ variation[18]. The de- 

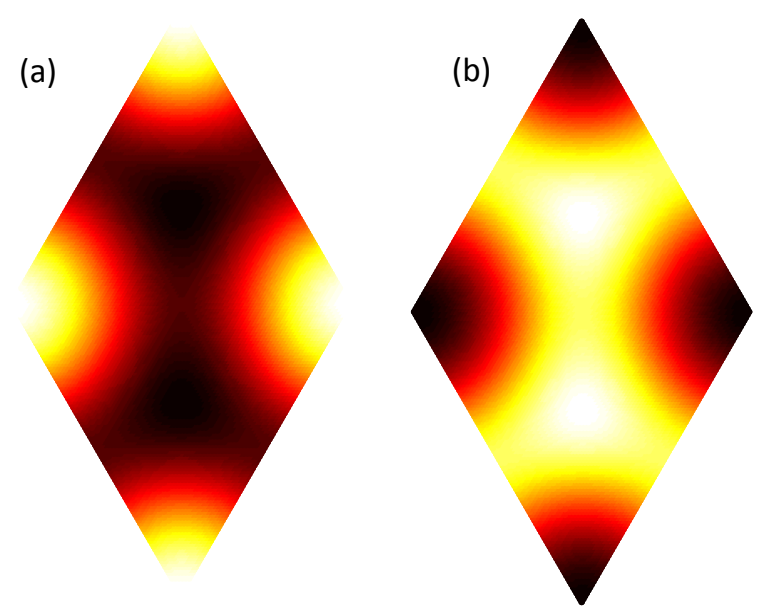

Figure 3.6: (a) The potential profile and (b) the corresponding low-energy occupied wave function difference. The lighter regions the difference is positive and darker regions represent the negative wave function difference.

viation from linear correction starts to be visible from supercells of $a \sim 6 \mathrm{~nm}$, and becomes around $44 \%$ error in $a \sim 15(\mathrm{~nm})$. we conclude that the wavefunction and therefore electron-hole puddles regions will be the same as the potential landscape for $a \leq 6$ and therefore electron-hole puddles will form for $\overline{\mathrm{C}} \mid h$-BN supercell which indicate the local change in the underlying potentials, and for larger supercells the the shape of potential landscape and charge density start to become different from one another, but still density will resemble the potential to a good degree.

One point worth mentioning here is that in Eq. 3.8 we have put $\lambda_{m}^{(o)}=0$ because for small supercells the gap in the system vanishes. For $1 \times 1 \overline{\mathrm{C}} \mid h$-BN cells the gap is large, but when the system rotates the gap disappears. This can be attributed to the fact that in large supercells we have regions similar to (a), (b), and (c) configurations, but when the size of a unit cell decreases it is not obvious which configuration the supercell will map into in this limit. Therefore if we want to find the small supercells correspond to the large supercells then the small supercell will be a superposition of $1 \times 1$ unitcells of $\overline{\mathrm{C}} \mid h$-BN. Now in this situation the $\lambda_{m}^{(o)}$ should be averaged over them. In three above mentioned unitcells, the (b) and (c) configurations are different with (a) unitcell because the mass term has opposite sign in two cases, which leads to dissapearence of $\lambda_{m}^{(o)}$ and that is the reason that in Fig 3.5 the gap is zero for $a=0$.

\subsection{Graphene on molybdenum disulfide}

Calculations have been performed of graphene on molybdenum disulfide $\left(\mathrm{MoS}_{2}\right)$, in order to determie wether the formation of electron-hole puddles in graphene on 
$h$-BN is caused by the in-plane polar covalent bonding character in the $h$-BN sheet. $\mathrm{MoS}_{2}$ is a semiconductor with a hexagonal layered structure and a monolayer unit cell consisting of two Sulfur atoms and one Molybdenum atom. The layers in the crystal are weakly by van der Waals forces and can be cleave in a similar fashion as $h$-BN. The outside of a $\mathrm{MoS}_{2}$ monolayer consists of only Sulfur atoms, therefore if graphene is placed on top of $\mathrm{MoS}_{2}$ it is not affected by lateral polar covalent bonds as in $h$-BN. The lattice mismatch of $22 \%$ with graphene is much larger than for $h$-BN. In order to compare $\overline{\mathrm{C}} \mid \mathrm{MoS}_{2}$ with $\overline{\mathrm{C}} \mid h$-BN we constructed an aligned $5 \times 5$ graphene on $4 \times 4 \mathrm{MoS}_{2}$ supercell, because the rotated $\overline{\mathrm{C}} \mid h$-BN supercells have different structures than local aligned commensurable systems. Similar interface dipole layer calculations as for $\overline{\mathrm{C}} \mid h$-BN have been performed. The LDA equilibrium binding distance $d_{\text {eq }}$ between $\mathrm{MoS}_{2}$ and graphene sheet is $3.34 \AA$ the potential step across the interface is $\Delta V=0.26 \mathrm{eV}$, and therefore the potential step is larger in $\overline{\mathrm{C}} \mid \mathrm{MoS}_{2}$ in comparison with $\overline{\mathrm{C}} \mid h$-BN. However upon translation of the graphene lattice over the $\mathrm{MoS}_{2}$ substrate, a negligible change in the binding distances and the strength of the interface potential step were found. The chemical selectivity for the carbon atom to bond to the Nitrogen or Boron atoms is lost for $\overline{\mathrm{C}} \mid \mathrm{MoS}_{2}$ systems, since the interactions are dominated by the homogeneous top Sulfur layer and graphene sheet. Therefore $\overline{\mathrm{C}} \mid h$-BN, intrinsic electron-hole puddles in $\overline{\mathrm{C}} \mid \mathrm{MoS}_{2}$ are not expected.

\subsection{Conclusion}

In conclusion, we have found that an interface dipole layer is formed between graphene and $h$-BN. The size and direction of the accompanying potential step are dependent on the precise binding configuration. Since in experiment $\overline{\mathrm{C}} \mid h$-BN forms an incommensurable structure in which the local binding configuration gradually changes, we expect slowly varying dipole layer and a corresponding electrostatic potential landscape. The electrostatic potential will be only partially screened because of the low density of states in graphene around the Fermi level. The Dirac cone will be moved following the direction of the local electrostatic potential of the inhomogeneous dipole layer. The result is a redistribution of charge in-plane, causing intrinsic electron-hole puddles to appear in graphene when placed on hexagonal boron nitride. The electron-hole puddle formation is caused by the polar covalent character of the bonds in $h$-BN, therefore we predict that no intrinsic electron-hole puddles will be formed when graphene in placed on a homogeneous material like $\mathrm{MoS}_{2}$. 


\section{Increasing the effective spin-orbit} coupling in graphene by hybridization with transition metal dichalcogenide substrate

Graphene was the first material proposed to be a topological insulator. Because its spin-orbit coupling (SOC) is so weak, experiments with it would have to be carried out at impracticably low temperatures. We use density functional theory calculations to explore the possibility of inducing a larger effective SOC in graphene by letting it hybridize with layered semiconducting $\mathrm{MX}_{2}$ transition metal dichalcogenide which, with $\mathrm{M}=\mathrm{Mo}$ or $\mathrm{W}$ and $\mathrm{X}=\mathrm{S}$, Se or Te, have sizeable spin-orbit splittings at the $\mathbf{K}$ point. We generalize the phenomenological low energy Hamiltonian proposed by Kane and Mele for graphene to describe the electronic structure modified by interaction with a substrate and use it to show that $\mathrm{MX}_{2} \mid$ graphene $\mid \mathrm{MX}_{2}$ sandwiches with inversion and reflection symmetry are $\mathbb{Z}_{2}$ topological insulators with small gaps but graphene $\mid \mathrm{MX}_{2}$ bilayers are normal insulators. Applying external pressure does not change their topological invariants.

\subsection{Introduction}

The discovery of topological insulators has changed our perception of the "triviality" of the insulating state in band insulators. Insulators can be categorized by "topological invariants" and since these invariants are not continuous, interesting phenomena occur when they have to change. The first group of these invariants was found in describing the quantum Hall effect for electrons confined in strong magnetic fields 
[36, 83, 26]. Kane and Mele [29, 30] proposed another class of "topological insulators" for systems with time-reversal symmetry where the invariant can have two values. Systems which are topologically nontrivial in the presence of time-reversal symmetry are called $\mathbb{Z}_{2}$ topological insulators (TI) $[29,30,25,32,84,85]$. The non-trivial behaviour is caused by the spin-orbit interaction (SOI).

Kane and Mele showed that the spin-orbit coupling (SOC) in graphene that opens a gap at the Dirac point could give rise to topologically protected spin-polarized edge states $[29,30]$. A number of studies indicated that the intrinsic SOC of graphene is so weak that for typical values of Rashba coefficient, temperatures of order $\sim 0.01 \mathrm{~K}$ $[86,87,88]$ would be required to observe these states though subsequent calculations have revised this temperature upwards to $<0.6 \mathrm{~K}$ (corresponding to a gap of $<50 \mu \mathrm{eV}$ ) $[89,90]$. It has been proposed to increase the SOC in graphene by "decorating" it with heavy adatoms [91,92,93] or by making it interact with a suitable substrate. A number of different substrate materials have been studied theoretically as possible candidates, for example $\mathrm{Bi}_{2} \mathrm{Se}_{3}$ which is itself a TI [94]; the transition metal dichalcogenides (TMD) $[95,96,97,98]$; the layered BiTeX compound with $X$ $=\mathrm{Cl}$, Br, or I [99]. This work shows that the spin-orbit splitting of graphene can be enhanced by three orders of magnitude on the TMD substrates and that around the graphene Dirac point a band inversion occurs that has been interpreted to indicate that the graphene bands in these systems have a non-trivial topological character. The TMDs have hexagonal lattices, can be exfoliated into single layers [34] and can be composed of heavy metals and chalcogens that give rise to large spin-orbit splittings of their energy bands [100]. If the graphene $p_{z}$ orbitals can be made to hybridize with such a substrate, a larger SOC might be induced at the Dirac point. The lack of a coherent and complete low-energy model to describe the band structure of graphene on TMD substrates as well as the importance of spatial symmetries such as reflection and inversion symmetry prompted us to address this problem.

We used a combination of density functional theory calculations and phenomenological tight binding Hamiltonians to study the electronic structure of graphene $(\overline{\mathrm{C}})$ on monolayers of the six TMD $\mathrm{MX}_{2}$ compounds with $\mathrm{M}=\mathrm{Mo}$ or $\mathrm{W}$ and $\mathrm{X}=\mathrm{S}$, Se or $\mathrm{Te}$. The $\mathrm{MX}_{2}$ materials resemble graphene in that they have a layered hexagonal structure where the carbon atoms of one graphene sublattice are replaced with $\mathrm{M}$ atoms while those of the other sublattice are replaced with a pair of $X$ atoms positioned symmetrically on either side of the $\mathrm{M}$ atom plane. Each $\mathrm{M}$ atom is located at the centre of a trigonal $X_{6}$ prism with threefold rotation symmetry. Besides studying asymmetric (AS) $\overline{\mathrm{C}} \mid \mathrm{MX}_{2}$ bilayers, we constructed $\mathrm{MX}_{2}|\overline{\mathrm{C}}| \mathrm{MX}_{2}$ trilayers with reflection symmetry (RS) as well as trilayers with inversion symmetry (IS).

The plan of the paper is as follows. In Sect. 4.2.1 we describe how the AS, RS, and IS supercells were constructed with minimal lattice mismatch and three-fold rotational symmetry. In Sect. 4.2.2 we describe the most important computational details and in Sect. 4.2.3 we present the calculated work functions and summarize how the graphene bands are aligned with the $\mathrm{MX}_{2}$ valence and conduction bands. In Sect. 4.3 we derive a phenomenological low energy Hamiltonian to inter- 
pret our first-principles electronic structures by considering a toy model of graphene on hexagonal boron nitride. This effective Hamiltonian is then applied to the AS bilayers, RS and IS trilayers and simplified in each case. By evaluating the eigenvalues and vectors and with the help of phase space arguments, we distinguish the topological insulating regions of parameters. In Sect. 4.4 the theoretical model is applied to first-principle electronic structures of AS, RS, and IS graphene-MX $\mathrm{X}_{2}$ systems and we present our fitting results and analysis of the bands.

\subsection{Calculations}

The weak "van der Waals" (vdW) interaction between graphene and $\mathrm{MX}_{2}$ and the chemical homogeneity of the $X$ layer seen by graphene lead us to expect at best a weak preference for the alignment of the two lattices and a negligible gap-opening from sublattice symmetry breaking. Even graphene and the isomorphic hexagonal boron nitride ( $h$-BN) whose lattice mismatch is only $1.8 \%$ interact so weakly that it is not energetically favourable to "lock" in their lattices [101, 56]. In recent work [52] we studied how incommensurability and rotational misalignment affect the band gap induced in graphene by the sublattice symmetry breaking interaction with a hexagonal boron nitride ( $h$-BN) substrate that was originally predicted for rotationally aligned commensurable structures [18]. Though greatly reduced, a finite band gap was found to survive incommensurability as long as the lattices were orientationally aligned. However, even a small deviation from perfect rotational alignment was sufficient to suppress it completely [52]. Because of the homogeneous composition of the outer $\mathrm{X}$ layers in $\mathrm{MX}_{2}$ compared to the partly ionic $\mathrm{B}^{+}$and $\mathrm{N}^{-}$in $h$ - $\mathrm{BN}$, we expect any sublattice symmetry breaking interaction will be substantially weaker. For these reasons, we do not expect that a rotation of the graphene and $\mathrm{MX}_{2}$ lattices will greatly influence the electronic properties. In particular, we expect that in the absence of spin-orbit coupling even a small deviation from perfect rotational alignment will suppress any substrate-induced band gap. The spin-orbit splittings that we will find in our calculations will be directly attributable to substrate-induced SOC. In the absence of energetics favouring alignment, the findings of the present work should be applicable to most experimentally accessible systems unless of course they are accidentally aligned.

\subsubsection{Constructing the supercells}

To construct $\overline{\mathrm{C}} \mid \mathrm{MX}_{2}$ unit cells with minimal mismatch, we take advantage of the freedom to rotate the substrate with respect to the graphene layer. For a graphene $\left(\mathrm{MX}_{2}\right)$ lattice with primitive translation vectors $\mathbf{a}_{1}$ and $\mathbf{a}_{2}\left(\mathbf{b}_{1}\right.$ and $\left.\mathbf{b}_{2}\right)$, any lattice vector $\mathbf{t}$ can be expressed as

$$
\mathbf{t}_{\overline{\mathrm{C}}}=n_{1} \mathbf{a}_{1}+n_{2} \mathbf{a}_{2} ; \mathbf{t}_{\mathrm{MX}}=m_{1} \mathbf{b}_{1}+m_{2} \mathbf{b}_{2}
$$


Table 4.1: Properties of $\mathrm{MX}_{2}$ monolayers and $\overline{\mathrm{C}} \mid \mathrm{MX}_{2}$ bilayers calculated with the optB88 functional. Shown are the in-plane lattice constants $a$, the equilibrium separation between the two $X$ planes $c_{X}$, and between graphene and the neighbouring $X$ plane $d_{\text {eq }}$, all in Angstroms. $\eta$ is the substrate strain for supercells containing $N_{\mathrm{MX}_{2}}$ $\left(N_{2}\right)$ primitive unit cells of $\mathrm{MX}_{2}$ and $N_{\overline{\mathrm{C}}}$ unit cells of graphene with the lattices rotated with respect to one another through an angle $\theta . E_{b}$ is the LDA equilibrium binding energy between graphene and the substrate per graphene unit cell in meV. $\Delta v(\mathrm{meV})$ and $\Delta c(\mathrm{meV})$ are the SOC induced splitting of the $\mathrm{MX}_{2}$ valence and conduction bands indicated in Fig. 4.3. $W_{\mathrm{MX}_{2}}(\mathrm{eV})$ is the workfunction of an $\mathrm{MX}_{2}$ monolayer, $W_{\overline{\mathrm{C}} \mid \mathrm{MX}_{2}}(\mathrm{eV})$ that of a $\overline{\mathrm{C}} \mid \mathrm{MX}_{2}$ bilayer.

\begin{tabular}{|c|c|c|c|c|c|c|c|c|c|c|c|c|}
\hline & $a(\AA)$ & $c_{\mathrm{X}}(\AA)$ & $\eta(\%)$ & $N_{2}$ & $N_{\overline{\mathrm{C}}}$ & $\theta$ & $\Delta v$ & $\Delta c$ & $d_{\mathrm{eq}}$ & $E_{b}$ & $W_{\mathrm{MX}_{2}}$ & $W_{\overline{\mathrm{C} \mid \mathrm{MX}}}$ \\
\hline $\mathrm{MoS}_{2}$ & 3.16 & 3.14 & 2.0 & 12 & 19 & 6.6 & 146.64 & 2.72 & 3.35 & -44.9 & 6.01 & 4.74 \\
\hline $\mathrm{MoSe}_{2}$ & 3.30 & 3.35 & 1.1 & 4 & 7 & 19.1 & 185.81 & 23.21 & 3.44 & -43.0 & 5.53 & 4.73 \\
\hline $\mathrm{MoTe}_{2}$ & 3.52 & 3.62 & 1.6 & 9 & 19 & 23.4 & 218.59 & 39.19 & 3.65 & -40.8 & 5.22 & 4.75 \\
\hline $\mathrm{WS}_{2}$ & 3.17 & 3.15 & 2.0 & 12 & 19 & 6.6 & 418.22 & 39.52 & 3.36 & -43.9 & 5.78 & 4.75 \\
\hline $\mathrm{WSe}_{2}$ & 3.30 & 3.37 & 1.1 & 4 & 7 & 19.1 & 454.03 & 47.11 & 3.45 & -42.3 & 5.31 & 4.72 \\
\hline $\mathrm{WTe}_{2}$ & 3.53 & 3.64 & 1.6 & 9 & 19 & 23.4 & 472.66 & 63.29 & 3.64 & -40.6 & 4.98 & 4.74 \\
\hline
\end{tabular}

where $n_{i}$ (and $m_{i}$ ) are integers. The primitive vectors are $\mathbf{a}_{1(2)}=\frac{a}{2}(\sqrt{3} \hat{x} \pm \hat{y})$ for graphene and $\mathbf{b}_{1(2)}=\frac{b}{2}(\sqrt{3} \hat{x} \pm \hat{y})$ for $\mathrm{MX}_{2}$. The carbon $A$ and $B$ sublattices are related by $\tau_{\mathrm{B}}=\tau_{\mathrm{A}}+\boldsymbol{\delta}_{\overline{\mathrm{C}}}$, the $\mathrm{M}$ and $\mathrm{X}$ sublattices by $\boldsymbol{\tau}_{\mathrm{X}}=\tau_{\mathrm{M}}+\boldsymbol{\delta}_{\mathrm{MX}}$ where $\boldsymbol{\delta}_{\overline{\mathrm{C}}}=$ $\left(\mathbf{a}_{1}+\mathbf{a}_{2}\right) / 3$ and $\boldsymbol{\delta}_{\mathrm{MX}_{2}}=\left(\mathbf{b}_{1}+\mathbf{b}_{2}\right) / 3$. We determine commensurable supercells with a tolerance expressed in terms of the maximum allowed substrate strain $\eta$ as $\mid t_{\overline{\mathrm{C}}}^{*}-$ $t_{\mathrm{MX}_{2}}^{*} \mid \leq \eta t_{\mathrm{MX}_{2}}^{*}$. Lattice vectors satisfying this condition are described by the integers $\left(n_{1}^{*}, n_{2}^{*} ; m_{1}^{*}, m_{2}^{*}\right)$. The supercell thus defined contains

$$
N_{\overline{\mathrm{C}}}=n_{1}^{* 2}+n_{1}^{*} n_{2}^{*}+n_{2}^{* 2}
$$

unit cells of graphene and

$$
N_{\mathrm{MX}_{2}}=m_{1}^{* 2}+m_{1}^{*} m_{2}^{*}+m_{2}^{* 2}
$$

unit cells of $\mathrm{MX}_{2}$. Choosing $b^{\prime}=b t_{\overline{\mathrm{Cr}}}^{*} / t_{\mathrm{MX}_{2}}^{*}$ as the strained lattice constant of the substrate, the primitive lattice vectors of the supercell will then be $\mathbf{T}_{1}=\mathbf{t}_{\overline{\mathrm{C}}}^{*}$ and $\mathbf{T}_{2}=R(\pi / 3) \mathbf{T}_{1}$ where $R(\pi / 3)$ is a rotation in the graphene plane. The vectors $\mathbf{t}_{\mathrm{C}}^{*}$ and $\mathbf{t}_{\mathrm{MX}_{2}}^{*}$ are not generally aligned so the substrate should be rotated by an angle

$$
\theta=\cos ^{-1} \frac{\mathbf{t}_{\overline{\mathrm{C}}}^{*} \cdot \mathbf{t}_{\mathrm{MX}}^{*}}{t_{\mathrm{C}}^{*} t_{\mathrm{MX}_{2}}^{*}} .
$$

A typical supercell of $\overline{\mathrm{C}} \mid \mathrm{MX}_{2}$ that contains $\sqrt{19} \times \sqrt{19}$ unit cells of graphene and $\sqrt{12} \times \sqrt{12}$ unit cells of $\mathrm{WS}_{2}$ is illustrated in Fig. 4.1.

It will turn out to be useful to construct $\mathrm{MX}_{2}|\overline{\mathrm{C}}| \mathrm{MX}_{2}$ sandwiches with well defined symmetries. To do so we use the remaining freedom to displace the two lattices with respect to one another by an arbitrary in-plane vector to construct an 


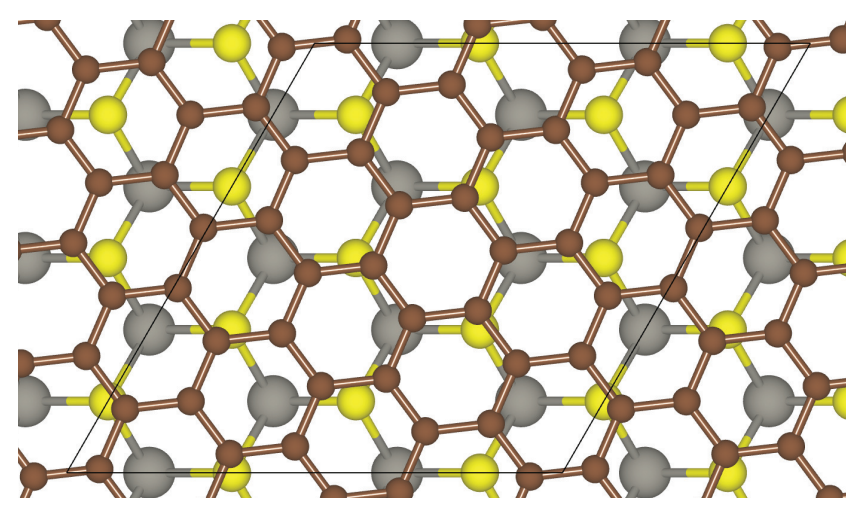

Figure 4.1: Top view of the unit cell of $\overline{\mathrm{C}} \mid \mathrm{WS}_{2}$, with three-fold rotation symmetry $C_{3}$ about the center of the unit cell. The small brown spheres are carbon atoms; the grey spheres indicate tungsten atoms and the yellow spheres represent sulphur atoms. This unit cell with $\left(n_{1}^{*}, n_{2}^{*} ; m_{1}^{*}, m_{2}^{*}\right)=(3,2 ; 2,2)$ contains 38 carbon, 12 tungsten, and 24 sulphur atoms. The relative rotation angle is $6.6^{\circ}$.

asymmetric (AS) bilayer with threefold rotation symmetry. Starting with supercells with one carbon atom and one $\mathrm{M}$ atom at the origin, we displace all of the atoms in the graphene layer by $\boldsymbol{\Delta}_{\overline{\mathrm{C}}}=\left(\mathbf{T}_{1}+\mathbf{T}_{2}\right) / 2-2 \boldsymbol{\delta}_{\overline{\mathrm{C}}}$ and in the $\mathrm{MX}_{2}$ layer by $\boldsymbol{\Delta}_{\mathrm{MX}_{2}}=\left(\mathbf{T}_{1}+\mathbf{T}_{2}\right) / 2-2 \boldsymbol{\delta}_{\mathrm{MX}_{2}}$ to align the threefold rotation axes. The center of the supercell then coincides with the center of a graphene hexagon which is an inversion center for graphene. We can then construct reflection symmetric (RS) or inversion symmetric (IS) trilayers with three-fold rotation symmetry by reflecting the $\mathrm{MX}_{2}$ layer in the graphene plane or inverting it with respect to the center of inversion symmetry.

This choice allows us to compare different $\overline{\mathrm{C}} \mid \mathrm{MX} \mathrm{X}_{2}$ bilayers whereby extraneous contributions to the electronic properties from different atomic geometries are suppressed. For some choices of supercells, the high symmetry $\mathbf{K}$ point in the Brillouin zone of graphene maps into the $\mathbf{K}, \mathbf{K}^{\prime}$ or $\boldsymbol{\Gamma}$ points in the Brillouin zone of the supercell. Since all of these points are compatible with $C_{3}$ symmetry, choosing the supercells to have this symmetry will help us keep the gaps close to the original graphene $\mathbf{K}$ point.

\subsubsection{Computational details}

To determine reasonable equilibrium geometries of $\mathrm{MX}_{2}$ monolayers, we used firstprinciples density functional theory (DFT) calculations within the framework of the projector augmented wave (PAW) method [8] as implemented in the Vienna Abinitio Simulation Package (VASP) code $[58,59,9]$. Because the interaction in weakly $\mathrm{vdW}$ bonded systems is difficult to describe within DFT, we consider three different functionals: the local density approximation (LDA) as parameterized by Perdew 
and Zunger [3], the PBE generalized gradient approximation (GGA) [6], and a van der Waals density functional $[78,102,103]$. Though it generally overestimates chemical interactions, the LDA gives a reasonable description of the binding energy and equilibrium separation of $\overline{\mathrm{C}} \mid$ metal $[104,105]$ and $\overline{\mathrm{C}} \mid h$-BN interfaces [18, 45, 52]. GGA often gives a good description of chemisorption but fails to capture physisorption. Local or semi-local functionals lack vdW interactions which play an important role in physisorption [106] and in the bonding of layered materials [107]. In this paper we use one of the recently developed and implemented van der Waals density functionals (vdW-DF) [78, 102, 103], and compare the results to those obtained with LDA. In the vdW-DF, the exchange-corrrelation functional is split up as $E_{\mathrm{xc}}=$ $E_{\mathrm{x}}+E_{\mathrm{c}}^{\mathrm{vdW}}+E_{\mathrm{c}}^{\mathrm{loc}}$, where $E_{\mathrm{c}}^{\mathrm{vdW}}$ describes non-local electron-electron correlations and $E_{\mathrm{c}}^{\text {loc }}$ local correlations. For $E_{\mathrm{c}}^{\mathrm{vdW}}$ and $E_{\mathrm{c}}^{\text {loc }}$ we use the vdW kernel developed by Dion et al. [78] and the LDA correlation [108], respectively. For the exchange part $E_{\mathrm{x}}$, we use the optB88 functional [103]. The resulting optB88-vdW-DF functional gives a satisfactory description of the lattice parameters and binding energy of graphite.

Electronic structure codes like VASP use a plane wave basis to very efficiently optimize geometrical and electronic properties at the price of imposing periodic boundary conditions. Introducing the supercell lattice vector $\mathbf{T}_{3}$ perpendicular to $\mathbf{T}_{1}$ and $\mathbf{T}_{2}$ to define the position of the periodic images in all three directions, we simulate single layers by adding a "vacuum" spacer layer of sufficient thickness $d_{\mathrm{vac}}$ between the images that the interaction between them can be neglected. If the $\mathrm{X}-\mathrm{X}$ distance in $\mathrm{MX}_{2}$ is $c_{\mathrm{X}}$, then for an $\mathrm{MX}_{2}$ monolayer the supercell size in the perpendicular direction is $d_{\mathrm{vac}}+c_{\mathrm{X}}$.

We construct $\mathrm{MX}_{2}|\overline{\mathrm{C}}| \mathrm{MX}_{2}$ sandwiches with IS and RS with the graphene plane situated at height zero so that the center of inversion through which the $C_{3}$ rotation axis passes is at $\mathbf{r}_{c}=\left(\mathbf{T}_{1}+\mathbf{T}_{2}\right) / 2$. The position of every $\mathrm{M}$ and $\mathrm{X}$ atom can be expressed as $\mathbf{r}_{\mathrm{M}(\mathrm{X})}=\alpha \mathbf{T}_{1}+\beta \mathbf{T}_{2}+\gamma \mathbf{T}_{3}$ where $0 \leq \alpha, \beta<1$ and we choose $\gamma<0$ for the AS bilayer. For the IS trilayers, each $\mathrm{M}$ and $\mathrm{X}$ atom in the first layer is inverted through the center of the supercell (inversion center). The positions of the $\mathrm{M}$ and $\mathrm{X}$ atoms belonging to the second layer will be $\mathbf{r}_{M(X)}^{I}=(1-\alpha) \mathbf{T}_{1}+(1-\beta) \mathbf{T}_{2}-\gamma \mathbf{T}_{3}$. This supercell has both inversion and threefold symmetry around its center and the point group associated with it is $S_{6}$. For the RS trilayers, we only need to reflect the $\mathbf{T}_{3}$ component of the atomic positions so $\mathbf{r}_{M(X)}^{R}=\alpha \mathbf{T}_{1}+\beta \mathbf{T}_{2}-\gamma \mathbf{T}_{3}$ and the associated point group will be $C_{3 h}$.

A plane-wave basis with a cutoff energy of $600 \mathrm{eV}$ was used in combination with the equivalent of a $\sim 36 \times 36 \times 1$ k-point grid in a $1 \times 1$ graphene unit cell (Table 4.1). Based on these structures we find the best supercell for the $\overline{\mathrm{C}} \mid \mathrm{MX}_{2}$ bilayer and adjust the in-plane lattice constant of the $\mathrm{MX}_{2}$ layer to graphene to avoid changing the electronic properties of graphene. With this new lattice constant, the structure of $\mathrm{MX}_{2}$ is again relaxed to minimize the energy. After these steps we obtained binding energies and separations of $\overline{\mathrm{C}} \mid \mathrm{MX} \mathrm{X}_{2}$ bilayers. The graphene cone and the $\Gamma, K$ and $\mathbf{M}$ high symmetry points were always explicitly included where the original $\mathbf{K}$ point in graphene maps into $\boldsymbol{\Gamma}$ or $\mathbf{K}\left(\mathbf{K}^{\prime}\right)$. For example, $\mathrm{WSe}_{2}$ and graphene are matched 


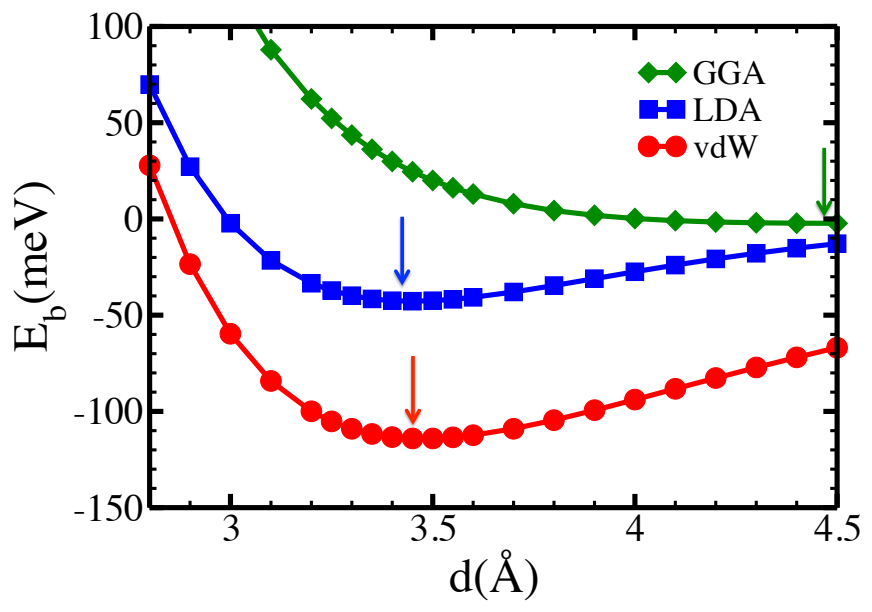

Figure 4.2: Binding energy per $\mathrm{C}_{2}$ unit cell for graphene on a $\mathrm{WSe}_{2}$ monolayer calculated with the GGA (green line, diamonds), LDA (blue line, squares), and vdW (red line, circles) functionals as a function of the layer separation $\mathrm{d}$.

to $\sim 1.1 \%$ by using a $2 \times 2$ supercell of $\mathrm{WSe}_{2}$ and a $\sqrt{7} \times \sqrt{7}$ supercell of graphene. For this $\overline{\mathrm{C}} \mid \mathrm{WSe}_{2}$ system, we use a $24 \times 24 \times 1$ k-point grid. The vacuum thickness was set to $15 \AA$ and a dipole correction applied to avoid artificial effects of image charges [81]. The electronic self-consistency criterion was set to $10^{-7} \mathrm{eV}$ during all of the calculations in order to obtain numerically well converged band gaps. The geometrical and electronic properties of monolayer $\mathrm{MX}_{2} \mathrm{TMDs}$ and $\overline{\mathrm{C}} \mid \mathrm{MX}_{2}$ bilayers are given in Table 4.1 .

The results are illustrated in Fig. 4.2 for $\overline{\mathrm{C}} \mid \mathrm{WSe}_{2}$ using the LDA, GGA and vdW functionals. GGA gives essentially no bonding with an adsorption energy $E_{\mathrm{b}}=-3$ $\mathrm{meV} / \mathrm{C}_{2}$ at an equilibrium separation $d_{\mathrm{eq}}=4.5 \AA$. LDA gives a reasonable bonding with $E_{\mathrm{b}}=-42.3 \mathrm{meV} / \mathrm{C}_{2}$ at $d_{\mathrm{eq}}=3.45 \AA$. The optB88-vdW-DF gives $E_{\mathrm{b}}=-120$ $\mathrm{meV} / \mathrm{C}_{2}$ at $d_{\mathrm{eq}}=3.48 \AA$. Because little is known from experiment about the equilibrium separations, we err on the side of caution and use the vdW functional separations to study the effect of spin-orbit coupling.

In the PAW method, all-electron wave functions are derived from smooth pseudo wave functions by means of a linear transformation [8] allowing all physical properties to be evaluated using the latter. In the VASP implementation, the spin-orbit interaction is evaluated taking into account only the spherical part of the potential inside muffin tins surrounding the carbon nuclei. For "free" atoms in a large box, we calculated the spin-orbit splitting of the valence orbitals of all of the atoms treated in this paper and reproduced the splittings reported by Herman and Skillman [109]; in particular we find a $2 p_{\frac{3}{2}}-2 p_{\frac{1}{2}}$ splitting for carbon of $8.7 \mathrm{meV}$ in agreement with a recently reported value [90].

Because of the small size of the splittings at the $\mathbf{K}$ point of graphene, we carried 

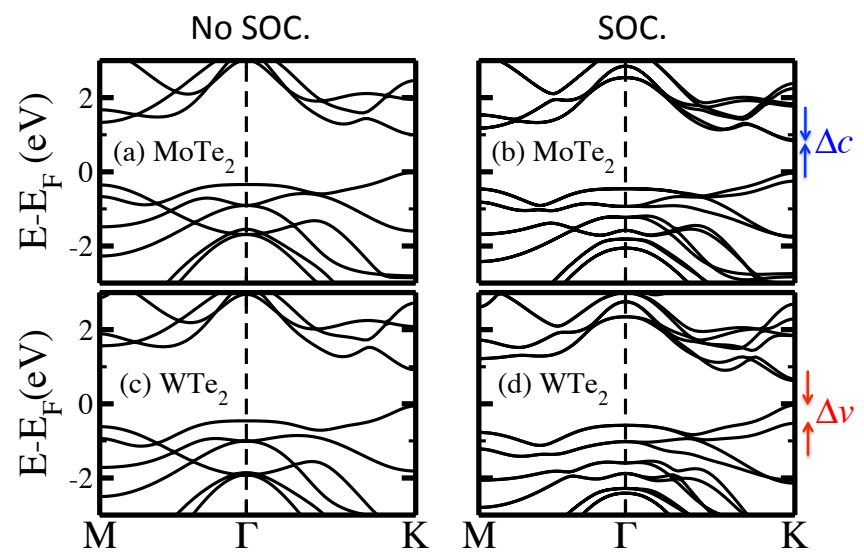

Figure 4.3: Band structures of $\mathrm{MoTe}_{2}(\mathrm{a}, \mathrm{b})$ and $\mathrm{WTe}_{2}(\mathrm{c}, \mathrm{d})$ without $(\mathrm{a}, \mathrm{c})$ and with $(\mathrm{b}, \mathrm{d})$ spin-orbit interactions. The spin-orbit splitting $\Delta v$ of the valence band and $\Delta c$ of the conduction band referred to in the text are indicated.

out numerical tests of the accuracy of the eigenvalue determination for a graphene monolayer. Without SOC, we can converge the eigenvalues so that at the $\mathbf{K}$ point they are (fourfold) degenerate to better than $10^{-6} \mathrm{eV}$. Including SOC, we find a gap of $0.022 \mathrm{meV}$ opens between states that are doubly degenerate to better than $10^{-6} \mathrm{eV}$. This gap is in agreement with the gaps reported using all electron methods $[89,90]$. We also reproduce the much larger SOC induced splitting of $\sim 9.31 \mathrm{meV}$ of the $\pi$ bands at the $\Gamma$ point found using the FLAPW method [90].

\subsection{3 $\mathrm{MX}_{2}$ band structure and band alignments}

The work functions of graphene and $\mathrm{MX}_{2}$ and the potential step at a $\overline{\mathrm{C}} \mid \mathrm{MX} \mathrm{X}_{2}$ interface determine where the Dirac point of graphene is situated in the $\mathrm{MX}_{2}$ band gap. The potential step is obtained from self-consistent calculations as the difference between the work functions $W_{\mathrm{MX}_{2}}$ of the ideal relaxed $\mathrm{MX}_{2}$ monolayer with its in-plane lattice constant adjusted to match that of graphene and that of the combined system $W_{\overline{\mathrm{C}} \mid \mathrm{MX}_{2}}$ as $\Delta V_{\mathrm{SCF}}=W_{\mathrm{MX}_{2}}-W_{\overline{\mathrm{C}} \mid \mathrm{MX}_{2}}[81,57]$.

In Fig. 4.4 we show the band structures of $\operatorname{MoTe}_{2}(\mathrm{a}, \mathrm{b})$ and $\mathrm{WTe}_{2}(\mathrm{c}, \mathrm{d})$ in the $\mathrm{M}-\boldsymbol{\Gamma}$ $\mathbf{K}$ direction without SOC $(\mathrm{a}, \mathrm{c})$ and with SOC $(\mathrm{b}, \mathrm{d})$. For monolayers, the band gap is direct and at the $\mathbf{K}$ point $[19,110,111,112]$. Replacing Mo with $\mathrm{W}$ is seen to increase the spin-orbit splitting $\Delta v$ of the top of the valence band at the $\mathbf{K}$ point substantially and that of the bottom of the conduction band $\Delta c$ less so [100]. $\Delta v$ and $\Delta c$ are shown schematically in Fig. 4.3 and numerical values are given in Table 4.1. These results show that the splitting of the valence and conduction band extrema depend mostly on the metal (M) and not on the chalcogen $(X)$ atom.

As shown in Fig. 4.4 and Table 4.1, the work function $W_{\mathrm{MX}_{2}}$ and the band gaps of 


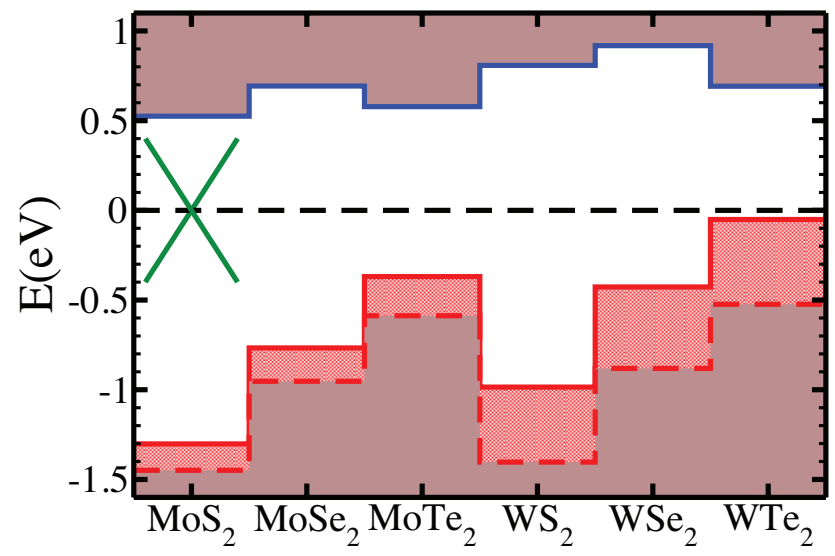

Figure 4.4: Schematic alignment of $\mathrm{MX}_{2}$ band structures with respect to the graphene Dirac point (dashed black line). The thick lines indicate, respectively, the top of the valence band (red) and the bottom of the conduction band (blue) for each compound. The light hatched region between the solid and dashed red lines is equal to the SO splitting $(\Delta v)$ in each $\mathrm{MX}_{2}$ layer. The (blue) conduction band splitting is not visible on this scale.

monolayer $\mathrm{MX}_{2}$ decrease in the sequence $\mathrm{S} \rightarrow \mathrm{Se} \rightarrow$ Te. Replacing Mo with $\mathrm{W}$ also lowers the work function of $\mathrm{MX}_{2}$ as shown in Table 4.1. Fig. 4.4 results from first principles calculations for $\overline{\mathrm{C}} \mid \mathrm{MX}_{2}$ bilayers and therefore includes also the dipole layer contribution [57]. The hatched region represents the valence band splitting of the $\mathrm{MX}_{2}$ layers, and we found that this splitting does not depend strongly on the $\mathrm{X}$ atoms since the valence band is dominated by $\mathrm{M}$ atom $d_{x^{2}-y^{2}}$ and $d_{x y}$ state character (for $\mathrm{MoS}_{2}$ we have $76 \% d_{x^{2}-y^{2}}, d_{x y}$ Mo character, only $20 \% \mathrm{~S}$ atom $p_{x}, p_{y}$ character and $4 \%$ from other orbitals [113]). This can be seen in Fig. 4.4 where the spin-orbit induced splitting of the valence and conduction bands is almost the same for all three chalcogens $S$, Se and Te for both the Mo and W compounds. For the valence band maximum at the $\mathbf{K}$ point, this can be understood in terms of the atom and orbital resolved character of these states having largely in-plane $d$-orbital $\left(d_{x^{2}-y^{2}}\right.$ and $\left.d_{x y}\right)$ character. The ratio of the free atom $d$ state spin orbit splitting, $0.7 \mathrm{eV}$ for $\mathrm{W}$ and $0.2 \mathrm{eV}$ for $\mathrm{Mo}$, is close to the ratio of the SO splitting of $\mathrm{WX}_{2}$ to that of $\mathrm{MoX}_{2}$ as given in Table 4.1.

\subsection{Constructing the model}

Before presenting detailed results of the ab-initio calculations for graphene on $\mathrm{MX}_{2}$, it will be useful to derive a simple model that will prove capable of capturing all of the essential features of the numerical results for the low energy electronic structure of graphene interacting with a generic substrate but in a form more suitable for 
intuitive understanding.

To describe the behaviour of the low energy $\pi$ bands of graphene around the Dirac point, we take hexagonal boron nitride $(\overline{\mathrm{C}} \mid h-\mathrm{BN})$ as a model substrate because of its simplicity. We start with a full Slater-Koster [10] LCAO (linear combination of atomic orbitals) model for a $\overline{\mathrm{C}} \mid h$-BN (graphene on hexagonal boron nitride) bilayer. In this representation, the $32 \times 32$ Hamiltonian takes the form

$$
H=\left(\begin{array}{cc}
\mathcal{G} & \mathcal{I} \\
\mathcal{I}^{\dagger} & \mathcal{S}
\end{array}\right)=\left(\begin{array}{ccc}
\mathcal{G}_{z} & \mathcal{G}_{I} & \mathcal{I}_{1} \\
\mathcal{G}_{I}^{\dagger} & \mathcal{G}_{s p^{2}} & \mathcal{I}_{2} \\
\mathcal{I}_{1}^{\dagger} & \mathcal{I}_{2}^{\dagger} & \mathcal{S}
\end{array}\right)
$$

where $\mathcal{G}$ represents the Hamiltonian of a monolayer of graphene on a chemically complete $p_{z} \oplus s p^{2}$ basis, $\mathcal{S}$ is the corresponding full $s p^{3}$ Hamiltonian of the $h$-BN monolayer "substrate", and $\mathcal{I}$ represents the interaction between the two monolayers. The graphene Hamiltonian $\mathcal{G}$ is decomposed into

$$
\mathcal{G}=\left(\begin{array}{cc}
\mathcal{G}_{z} & \mathcal{G}_{I} \\
\mathcal{G}_{I}^{\dagger} & \mathcal{G}_{s p^{2}}
\end{array}\right)
$$

where $\mathcal{G}_{z}$ and $\mathcal{G}_{s p^{2}}$ are, respectively, the $p_{z}$ and $s p^{2}$ orbital parts of the Hamiltonian and $\mathcal{G}_{I}$ is the interaction between these. In the absence of spin-orbit coupling $\mathcal{G}_{I}$ vanishes because of the odd (even) symmetry of the $p_{z}\left(s p^{2}\right)$ orbitals under reflection in the graphene plane. The SO interaction couples the $p_{z}$ state (with magnetic quantum number $m=0$ ) to the $p_{x}$ and $p_{y}$ states (with $m= \pm 1$ ). We can use Löwdin perturbation theory to downfold $\mathcal{G}$ into the subspace of $p_{z}$ orbitals as [114]

$$
\mathcal{G}_{z}^{D}(\varepsilon)=\mathcal{G}_{z}+\mathcal{G}_{I}\left(\varepsilon-\mathcal{G}_{s p^{2}}\right)^{-1} \mathcal{G}_{I}^{\dagger}
$$

Since we are interested in the eigenvalues $\varepsilon$ close to the Fermi energy at the $\mathbf{K}$ point, we can expand this equation around the unperturbed eigenvalues with $\varepsilon \sim 0$. The effective Hamiltonian for $p_{z}$ orbitals in the presence of SOC is

$$
\mathcal{G}_{z}^{D}(\varepsilon) \approx \mathcal{G}_{z}^{(2)}=\mathcal{G}_{z}-\mathcal{G}_{I} \mathcal{G}_{s p^{2}}^{-1} \mathcal{G}_{I}^{\dagger}
$$

to second order in the SOC $\mathcal{G}_{I}$, and is energy-independent. In the subspace of $p_{z}$ spin orbitals the wavefunction $\psi_{\mathbf{K}}$ can be represented as $\left(\varphi_{\mathbf{K} A \uparrow}, \varphi_{\mathbf{K} B \uparrow}, \varphi_{\mathbf{K} A \downarrow}, \varphi_{\mathbf{K} B \downarrow}\right)$ where $A$ and $B$ refer to the sublattices of graphene. In this representation the effective Hamiltonian at the $\mathbf{K}$ point will be [86]

$$
\mathcal{G}_{z}^{(2)}=\lambda_{\text {so }}\left(\begin{array}{rrrr}
1 & 0 & 0 & 0 \\
0 & -1 & 0 & 0 \\
0 & 0 & -1 & 0 \\
0 & 0 & 0 & 1
\end{array}\right)=\lambda_{\mathrm{so}} \sigma_{z} s_{z}
$$


where $\sigma$ and s are vectors of Pauli matrices, $\sigma_{z}$ representing states on the $A$ and $B$ sublattices of graphene and $s_{z}$ representing spin. If we define [10] $\alpha=3(s p \sigma) / 2$ and $\beta=3[(p p \sigma)-(p p \pi)] / 4$ then

$$
\lambda_{\mathrm{so}}=\frac{\xi^{2}}{4}\left(\frac{\varepsilon_{s}}{\varepsilon_{s} \varepsilon_{x y}-2 \alpha^{2}}-\frac{\varepsilon_{x y}}{\varepsilon_{x y}^{2}-4 \beta^{2}}\right)
$$

where $\varepsilon_{s}\left(\varepsilon_{x y}\right)$ is the energy of the $s\left(p_{x y}\right)$ orbital(s) with respect to that of the $p_{z}$ orbital, $(s p \sigma)$ is the $\sigma$ interaction between $s$ and $p$ orbitals, $(p p \sigma)$ and $(p p \pi)$ are the $\sigma$ and $\pi$ interactions, respectively, between $p$ orbitals on neighbouring carbon atoms in graphene, and $\xi$ is the conventional intrinsic spin-orbit coupling parameter that can be calculated from the $\Delta_{p}=p_{\frac{3}{2}}-p_{\frac{1}{2}}$ splitting for a carbon atom $\left(\xi=2 \Delta_{p} / 3\right)$. For graphene, $\lambda_{\text {so }}$ is called the intrinsic spin-orbit term [30, 86]. Eq. (4.9) shows that the intrinsic spin-orbit interaction in graphene can open a (tiny) gap at the $\mathbf{K}$ point $[87,86,88,89,90]$.

Even though the band gap of $h$-BN at the $\mathbf{K}$ point is $\sim 6 \mathrm{eV}$, the closest $s p^{2}$ bands are at least $6 \mathrm{eV}$ further away in energy [115] and only very weakly coupled by SOC. This means that we can again apply Löwdin perturbation theory to the $h$-BN $p_{z}$ orbital subspace, the only difference being that the energy $\varepsilon$ in (4.6) is a non-zero constant energy. Choosing the wavefunction representation for $h$-BN to be $\psi_{\mathbf{K}}=$ $\left(\phi_{\mathbf{K B} \uparrow}, \phi_{\mathbf{K N} \uparrow}, \phi_{\mathbf{K B} \downarrow}, \phi_{\mathbf{K N} \downarrow}\right)$ where $\mathrm{B}$ and $\mathrm{N}$ refer to boron and nitrogen, we derive the effective substrate $p_{z}$ Hamiltonian at the $\mathbf{K}$ point

$$
\mathcal{S}_{z}^{(2)}=\lambda_{o}^{(\mathcal{S})}+\lambda_{m}^{(\mathcal{S})} \sigma_{z}+\lambda_{\mathrm{so}}^{(\mathcal{S})} \sigma_{z} s_{z}+\lambda_{B}^{(\mathcal{S})} s_{z}+\mathbf{f}_{\mathbf{K}} \cdot \boldsymbol{\sigma}
$$

where $\sigma_{z}$ now represents states on the boron and nitrogen sublattices. $\lambda_{o}^{(\mathcal{S})}$ and the sublattice symmetry breaking term $\lambda_{m}^{(\mathcal{S})}$ are, respectively, the sum and difference of the boron and nitrogen $p_{z}$ orbital energies with respect to the graphene $p_{z}$ orbital energy, $\lambda_{\text {so }}$ is the "intrinsic" SOC term in $h$-BN and $\lambda_{B}^{(\mathcal{S})}$ represents a "pseudomagnetic" term in $h$-BN which originates from SOC when inversion symmetry in broken. The term $\mathbf{f}_{\mathrm{K}} \cdot \boldsymbol{\sigma}$ is added to generalize the substrate Hamiltonian to materials other than $h$-BN; in the present case $\mathbf{f}_{\mathbf{K}}=0$. We will return to this term in the Appendix and show how it changes the Hamiltonian.

\subsubsection{Asymmetric bilayers}

The graphene energy bands at the Dirac point are separated by several electron volts from the nearest $h$-BN bands and the interaction between the two sheets is weak. For convenience and simplicity, we place graphene on $h$-BN with its $A$ sublattice above the $\mathrm{B}$ sublattice and its $B$ sublattice above the $\mathrm{N}$ sublattice so the threefold rotation axes coincide and the bilayer has threefold rotation symmetry. We proceed to downfold the substrate interaction into the graphene effective $p_{z}$ Hamiltonian at the $\mathbf{K}$ point. We are interested in corrections to second order in $\mathcal{I}_{1}$ and $\mathcal{G}_{I}$, see 
Eq. (4.5). Calling the $28 \times 28$ block

$$
\left(\begin{array}{cc}
\mathcal{G}_{s p^{2}} & \mathcal{I}_{2} \\
\mathcal{I}_{2}^{\dagger} & \mathcal{S}
\end{array}\right)
$$

in (4.5) $H_{R}$, the effective Hamiltonian (4.8) becomes

$$
\mathcal{G}_{z}^{(2)}=\mathcal{G}_{z}-\left(\mathcal{G}_{I}, \mathcal{I}_{1}\right) H_{R}^{-1}\left(\begin{array}{c}
\mathcal{G}_{I}^{\dagger} \\
\mathcal{G}_{1}^{\dagger}
\end{array}\right)
$$

for $p_{z}$ orbitals of graphene in the presence of a substrate. $H_{R}$ cannot be inverted analytically so we use the smallness of the interlayer interactions $\mathcal{I}_{2}$ compared to inplane interactions to approximate the inverse. Using $(A+\eta B)^{-1} \approx A^{-1}-\eta A^{-1} B A^{-1}$ where $\eta$ is a small parameter, we write

$$
H_{R}^{-1} \approx\left(\begin{array}{cc}
\mathcal{G}_{s p^{2}}^{-1} & -\mathcal{G}_{s p^{2}}^{-1} \mathcal{I}_{2} \mathcal{S}^{-1} \\
-\mathcal{S}^{-1} \mathcal{I}_{2}^{\dagger} \mathcal{G}_{s p^{2}}^{-1} & \mathcal{S}^{-1}
\end{array}\right) .
$$

With this approximation for $H_{R}^{-1}$ and the Slater-Koster form for the tight-binding matrix elements in (4.5), we use MATHEMATICA [116] to evaluate (4.13) "analytically" and derive the Hamiltonian for graphene $p_{z}$ bands close to the Dirac point

$$
H_{\mathbf{K}}^{\mathrm{AS}}(\mathbf{q})=\hbar v_{F} \mathbf{q} \cdot \boldsymbol{\sigma}+\lambda_{m} \sigma_{z}+\frac{\lambda_{R}}{2}(\boldsymbol{\sigma} \times \mathbf{s})_{z}+\lambda_{\mathrm{so}} \sigma_{z} s_{z}+\lambda_{B} s_{z}
$$

where we now rename $\mathcal{G}_{z}^{(2)}$ as $H_{\mathbf{K}}^{\mathrm{AS}}$ and $\mathbf{q}$ is the wave vector measured from the $\mathbf{K}$ point, $\mathbf{q}=\mathbf{k}-\mathbf{K}$. Though we have explicit expressions for the parameters in (4.15) in terms of the microscopic Slater-Koster matrix elements, they are too complex to be reproduced here. We return to this below.

The first term $\hbar v_{F} \mathbf{q} \cdot \boldsymbol{\sigma}$ is the unperturbed $p_{z}$ orbital Hamiltonian where $v_{F}$ is the Fermi velocity for graphene bands. Although $v_{F}$ can be modified by interaction with the substrate, its value is not found to change significantly from its unperturbed value. The second term $\lambda_{m}$ is an effective mass that can originate from interlayer hopping between graphene and the substrate but can also contain contributions arising from the electrostatic potential from the interface dipole layer [52]. $\lambda_{\text {so }}$ corresponds to the Kane-Mele [30] intrinsic spin-orbit interaction in graphene (Eq. 4.9) but also contains contributions from the interaction with the substrate. $\lambda_{R}$ is the Rashba term which originates from reflection-symmetry breaking interactions and can be described as a Stark effect contribution to the Hamiltonian from an external electric field perpendicular to the graphene plane [30, 86]. So far the Hamiltonian is identical to that proposed by Kane and Mele [30]; the final term $\lambda_{B} s_{z}$ is new $[98,97]$. This Zeeman-like term shifts states with opposite spins in opposite directions in energy and can be regarded as an effective magnetic field that is caused by interaction with the substrate since it is absent in the graphene monolayer Hamilto- 


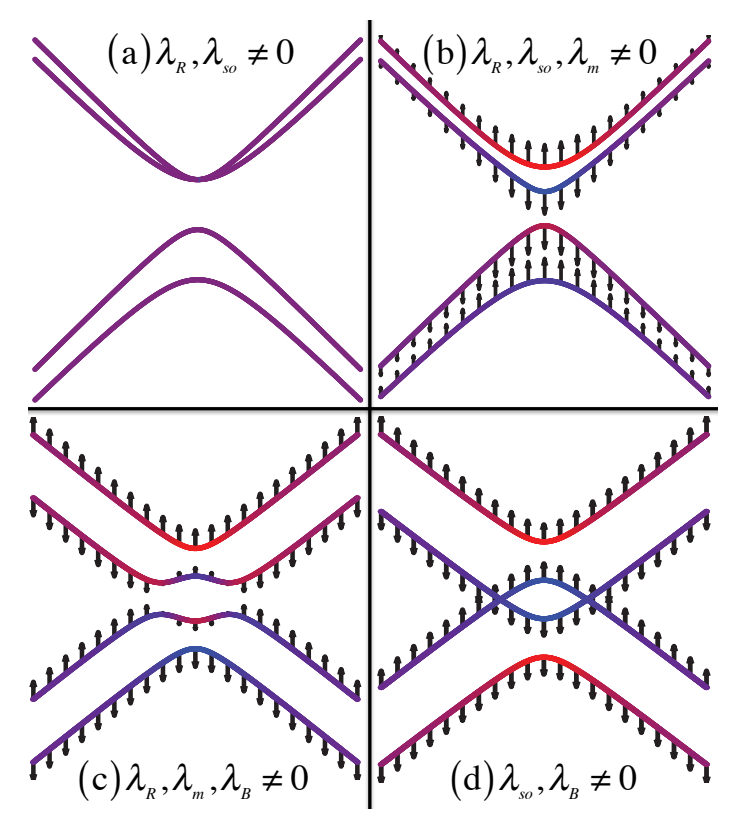

Figure 4.5: Representative band structures from equation (4.15) where $s_{z}$ is represented by a vertical arrow and the projection onto sublattices is give on a color scale; $A$ is red, $B$ is blue.

nian. We will return to it later and show that it does not have the symmetry of a real effective magnetic field but that of a pseudo-magnetic field (pseudo pseudovector = vector).

Because the $\lambda \mathrm{s}$ were derived from the full tight binding Hamiltonian at the $\mathbf{K}$ point, they depend on $\mathbf{q}$ via the Slater-Koster hopping parameters and so they are not truly constants. However, if we restrict ourselves to the band structure close to the $\mathbf{K}$ point, all of the corrections to these parameters can be neglected and we will be able to treat them as constants, the only explicit $q$ dependence coming from the $\hbar v_{F} \mathbf{q} . \boldsymbol{\sigma}$ linear term in (4.15). Because of their complexity and because we want to use the effective Hamiltonian for substrates more complicated than $h$-BN, we will treat (4.15) as a phenomenological Hamiltonian and the $\lambda \mathrm{s}$ as disposable constants to be determined experimentally or from first-principles calculations.

The eigenvalues of the $4 \times 4$ equation (4.15) at the $\mathbf{K}$ point are

$$
\begin{aligned}
& \varepsilon_{4}=-\lambda_{\text {so }}+\sqrt{\left(\lambda_{B}-\lambda_{m}\right)^{2}+\lambda_{R}^{2}} \\
& \varepsilon_{3}=-\lambda_{B}-\lambda_{m}+\lambda_{\text {so }} \\
& \varepsilon_{2}=\quad \lambda_{B}+\lambda_{m}+\lambda_{\text {so }} \\
& \varepsilon_{1}=-\lambda_{\text {so }}-\sqrt{\left(\lambda_{B}-\lambda_{m}\right)^{2}+\lambda_{R}^{2}}
\end{aligned}
$$


Depending on the values of the parameters, solutions of the Hamiltonian fall into the four regimes characterized by the spin characters of the bands sketched in Fig. 4.5 close to the $\mathbf{K}$ point. For the special case where $\lambda_{m}$ and $\lambda_{B}$ are both zero the bands contain the same amount of up and down spin character, Fig. 4.5(a). When $\lambda_{R}$ and $\lambda_{B}$ dominate, the minimum gap is not at the $\mathbf{K}$ point (see Fig. 4.5(c)) and band inversion occurs a distance

$$
q_{g}=\sqrt{2}\left|\lambda_{B}\right| \sqrt{\frac{2 \lambda_{B}^{2}+\lambda_{R}^{2}}{4 \lambda_{B}^{2}+\lambda_{R}^{2}}}
$$

from $\mathbf{K}$ where the size of the minimum gap is

$$
\Delta_{g}=\frac{2\left|\lambda_{B} \lambda_{R}\right|}{\sqrt{4 \lambda_{B}^{2}+\lambda_{R}^{2}}}
$$

\subsubsection{Reflection symmetric trilayers}

The derivation of Eq. (4.15) just sketched can be extended to include a layer of $h$-BN placed on either side of the graphene layer to form a $h$-BN $|\overline{\mathrm{C}}| h$-BN trilayer. Such a trilayer can be constructed to have reflection symmetry by mirroring the $z$-component of the $\mathrm{B}$ and $\mathrm{N}$ atoms of the first layer in the graphene layer. The equilibrium separation of the two $h$-BN layers is so large that their direct interaction can be neglected. We then extend Eq. (4.13) by adding the corresponding contributions of the second layer. Since the origin of the Rashba term is the electric field induced by $h$-BN (or any other substrate such as $\mathrm{MX}_{2}$ ) on graphene, then by construction the field produced by the second layer must give rise to an induced electric field with the opposite direction to the first, cancelling it so the corresponding RS Hamiltonian will not contain a Rashba term. The contribution to the mass term from dipole formation will also vanish but a hopping part remains. The effective Hamiltonian for the RS system will therefore be

$$
H_{\mathbf{K}}^{\mathrm{RS}}(\mathbf{q})=\hbar v_{F} \mathbf{q} \cdot \boldsymbol{\sigma}+\lambda_{m} \sigma_{z}+\lambda_{\mathrm{so}} \sigma_{z} s_{z}+\lambda_{B} s_{z}
$$

where we have neglected additional terms (discussed in the Appendix) induced by the $\mathbf{f}_{\mathbf{K}} \cdot \boldsymbol{\sigma}$ term in (4.11). The eigenvalues at the $\mathbf{K}$ point are then

$$
\begin{aligned}
& \varepsilon_{1}=-\lambda_{m}-\lambda_{\text {so }}+\lambda_{B} \\
& \varepsilon_{2}=\quad \lambda_{m}-\lambda_{\text {so }}-\lambda_{B} \\
& \varepsilon_{3}=-\lambda_{m}+\lambda_{\text {so }}-\lambda_{B} \\
& \varepsilon_{4}=\quad \lambda_{m}+\lambda_{\text {so }}+\lambda_{B}
\end{aligned}
$$

where the eigenvalue ordering depends on the parameter values. Without a Rashba term, band inversion occurs for large values of $\lambda_{B}>\lambda_{m}, \lambda_{\text {so }}$ when bands with opposite spins cross and the model predicts zero gap, Fig. 4.5(d). The crossing will 
happen at a distance

$$
q_{g}^{2}=\left(\frac{\lambda_{B}}{\hbar v_{F}}\right)^{2}\left(1-\frac{\lambda_{m}^{2}}{\lambda_{B}^{2}}\right)\left(1-\frac{\lambda_{s o}^{2}}{\lambda_{B}^{2}}\right)
$$

from the $\mathbf{K}$ point. When the reflection symmetry is broken so $\lambda_{R}$ is no longer zero, a gap opens around this point. Thus when $\lambda_{B}$ is dominant, the bands will cross at $q_{g}$ unless $\lambda_{R}$ opens a gap there.

\subsubsection{Inversion symmetric trilayers}

An approach similar to that sketched for RS trilayers can be applied to construct an effective Hamiltonian for inversion symmetric (IS) trilayers. Inversion and timereversal symmetries change $\mathbf{q} \rightarrow-\mathbf{q}$, inversion interchanges the graphene sublattices but does not change spins while time-reversal change spins but not sublattices. The operator for inversion symmetry can be expressed as $P=\sigma_{x}$ and time-reversal $\Theta=i s_{y} \hat{K}$ in our representation, where $\hat{K}$ is the complex conjugation operator. $\Theta$ invariance requires that $\lambda_{B}(\mathbf{K}) s_{z}=-\lambda_{B}\left(\mathbf{K}^{\prime}\right) s_{z}$, while $P$ requires $\lambda_{B}(\mathbf{K}) s_{z}=\lambda_{B}\left(\mathbf{K}^{\prime}\right) s_{z}$. If a system has both time-reversal $(\Theta)$ and inversion symmetries, then the Hamiltonian should be invariant under $P \Theta$ and $\lambda_{B}=0$. Now we can see that $\lambda_{B} s_{z}$ is odd under inversion, therefore $\lambda_{B}$ represents an induced magnetic field which is odd under inversion and therefore is a real vector and a pseudo magnetic field.

The mass term $\lambda_{m}$, pseudo magnetic terms $\lambda_{B}$ and terms induced by the $\mathbf{f}_{\mathbf{K}} \cdot \boldsymbol{\sigma}$ term in (4.11) (discussed in the Appendix) will be absent in IS systems. These terms are odd under inversion and therefore the contributions from two encapsulating layers (of $h$-BN or $\mathrm{MX}_{2}$, or whatever) with inversion symmetry will cancel each other out. The off-diagonal $\lambda_{R}$ term is also odd in our representation and will vanish globally though an effective electric field can be non-zero locally. Therefore, we expect that their values at the $\mathbf{K}$ point to be zero. Based on these considerations, the low energy Hamiltonian for IS systems around the $\mathbf{K}$ point has to be

$$
H_{\mathbf{K}}^{\mathrm{IS}}(\mathbf{q})=\hbar v_{F} \mathbf{q} \cdot \boldsymbol{\sigma}+\lambda_{\mathrm{so}} s_{z} \sigma_{z}
$$

with eigenvalues

$$
\varepsilon= \pm \sqrt{\left|\hbar v_{F} \mathbf{q}\right|^{2}+\lambda_{\text {so }}^{2}}
$$

and each value is doubly degenerate due to the $P \Theta$ symmetry of the IS system. For IS systems we have $[P, H(\mathbf{q})]=0$ and as a result each wave function is an eigenvector of the parity operator and bands have well defined parities. This property will be useful when we want to look at the topological nature of bands for IS systems.

\subsubsection{Phase space}

In an insulating system with time reversal symmetry, a $\mathbb{Z}_{2}$ topological invariant $\nu$ can be found by calculating the Pfaffian of the time-reversal operator for occupied bands 
at time-reversal fixed $\Gamma_{i}$ points [30, 32]. Calculating the Pfaffian from first-principles wavefunctions is, however, not well-formulated except for IS systems. Instead, we can understand the topological nature of bands by constructing the phase space of the effective Hamiltonian. Since $\nu$ can have two values corresponding to a trivial or a non-trivial (topological) insulator and the system can also have zero gap, the phase space will be divided into three regions, topological insulator regions (TI), normal insulating regions (NI), and metallic regions $(\mathrm{M})$ where the band gap is zero and the $\mathbb{Z}_{2}$ invariant cannot be defined. Since the invariant must change at a transition between TI and NI regions, the band gap in between should become zero. However a vanishing band gap does not necessarily imply that the invariant changes. Further support for this argument comes from the behaviour of occupied state wave functions in phase space. A $\mathbb{Z}_{2}$ topological insulator should show robust edge states where spin up and down are separated from each other, the total current is zero but the spin-current is not. The robustness of edge states means that backscattering is prohibited by a system having time-reversal symmetry. Kane and Mele formalized this argument for an arbitrary number of bands [29]. For our four band Hamiltonian with two states occupied, their argument can be translated into the mathematical condition that $\left\langle u_{\mathbf{K}}^{1}|\Theta| u_{\mathbf{K}}^{2}\right\rangle=0$ where $\left|u_{\mathbf{K}}^{1}\right\rangle$ and $\left|u_{\mathbf{K}}^{2}\right\rangle$ are the occupied cell periodic wave functions at the $\mathbf{K}$ point. That is, the occupied state Pfaffian for our model is Pf $=\left\langle u_{\mathbf{K}}^{1}|\Theta| u_{\mathbf{K}}^{2}\right\rangle$. Thus we can recognize TI, NI and M regions in phase space by looking at the band gaps and the quantity Pf, since Pf must be zero at the $\mathbf{K}$ point for TI systems with $C_{3}$. For the TI systems without $C_{3}$ symmetry the zero of Pfaffian can occur away from the $\mathbf{K}$ point . The last part of this argument comes from noting that the phase space region where all $\left|\lambda_{i}\right| \ll\left|\lambda_{\text {so }}\right|$ is TI while for $\left|\lambda_{i}\right| \ll\left|\lambda_{m}\right|$ it is NI and the Pfaffian must be zero in the TI region. We use the Berry curvature $\mathbf{B}(\mathbf{k})$ and the Berry potential $\mathbf{A}(\mathbf{k})$ to express the invariant $\nu$ as [117]

$$
\nu=\frac{1}{2 \pi}\left[\oint_{\partial(\mathrm{EBZ})} d \mathbf{k} \cdot \mathbf{A}(\mathbf{k})-\int_{\mathrm{EBZ}} B(\mathbf{k}) d \mathbf{k}\right] \bmod 2
$$

where EBZ is the effective Brillouin zone and $\partial(\mathrm{EBZ})$ is the boundary of the EBZ. In the low-energy regime the full Brillouin zone is $\mathbf{K} \oplus \mathbf{K}^{\prime}$, the EBZ will only contain $\mathbf{K}$ (or only $\mathbf{K}^{\prime}$ ) and the boundary is an infinitely large equilateral triangle with $\mathbf{K}$ at its center. The effective Hamiltonian has $C_{\infty}$ rotational symmetry for large $q$ and therefore its boundary is a circle, but the actual shape of $\partial(E B Z)$ is an equilateral triangle, however for $q \rightarrow \infty$ the integral of the Berry phase is the same for both. The Berry curvature for occupied bands of the Hamiltonian 4.15 decreases as a function of $q$ very fast $\left(B_{1}+B_{2} \approx \frac{\lambda_{m}}{\hbar v_{F} q^{3}}\right)$ and therefore the contribution of large $q$ is very small. Since the integral of Berry curvature can only have discrete values then the small changes in its large $q$ values will not change the result of this integral. The small changes in the rest of the EBZ is not strong enough to change the Berry curvature integral and the most important contribution comes form the neighbourhood of the 
(a)

(b)

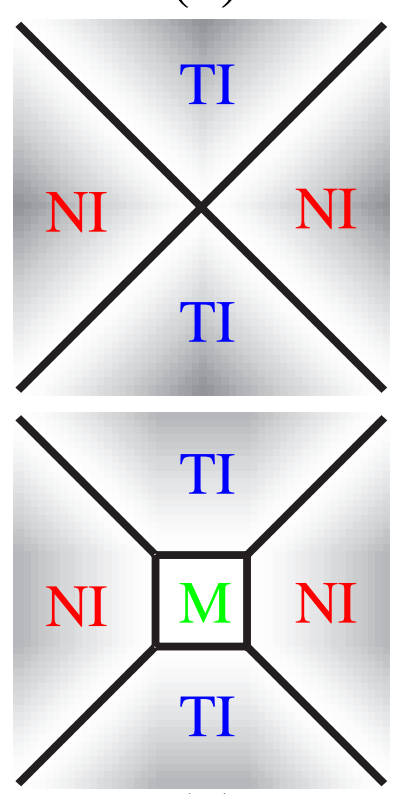

(c)

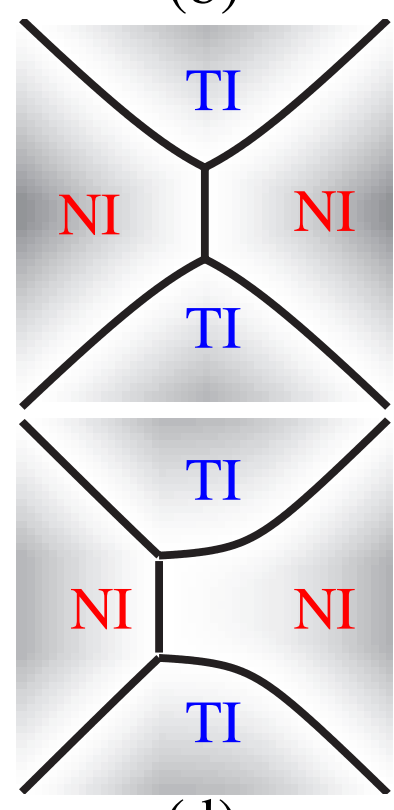

(d)

Figure 4.6: Various cross sections of the four dimensional phase space for Hamiltonian (4.15). The boundaries are determined by $\varepsilon_{3}-\varepsilon_{2}=0$. In panel (a) $\lambda_{R}=\lambda_{B}=0$, (b) $\lambda_{B}=0$, (c) $\lambda_{R}=0$, and in (d) all four parameters are non-zero. $\lambda_{m}$ is along the $x$ axis, $\lambda_{\text {so }}$ along the $y$ axis.

K point (where the bands are close to each other). We can write (4.24) as

$$
\nu=\frac{1}{2 \pi} \int \sum_{i=1,2} B_{i}(\mathbf{q}) d \mathbf{q}+1 \bmod 2
$$

where $B_{i}(\mathbf{q})$ is the Berry curvature of the $i^{t h}$ occupied band and +1 is the contribution of the boundary. For special cases this can be solved analytically and for $\left|\lambda_{i}\right| \ll\left|\lambda_{\text {so }}\right|$ it gives $\nu=1$ (TI) and for $\left|\lambda_{i}\right| \ll\left|\lambda_{m}\right|$ we found that $\nu=0$ (NI).

To summarize, we determine where the band gap vanishes in the phase space of the parameters of our Hamiltonian and then we check how different regions are connected to $\left|\lambda_{\text {so }}\right| \rightarrow \infty$ and $\left|\lambda_{m}\right| \rightarrow \infty$. If a point in phase space can be connected to $\left|\lambda_{m}\right| \rightarrow \infty$ without the gap vanishing then it must be NI, but if the gap vanishes then we must check if the Pfaffian is zero at that point and how it is connected to $\left|\lambda_{\text {so }}\right| \rightarrow \infty$. This inspection will partition phase space and allows us to predict the topological invariant of our system.

To find the boundaries between regions where $\nu$ can change, we solve $\varepsilon_{3}-\varepsilon_{2}=0$. For IS trilayers this equation simplifies to $\sqrt{\left|\hbar v_{F} \mathbf{q}\right|^{2}+\lambda_{\text {so }}^{2}}=0$. The Hamiltonian in 
(4.23) has a simple phase space, since the gap only vanishes when $\varepsilon$ is zero. This can only happen at $\mathbf{q}=0$ if $\lambda_{\mathrm{so}}=0$ which defines a point in phase space in which the system is gapless and $\nu$ cannot be defined. There is no NI region for the IS trilayer because $\lambda_{m}$ is strictly zero; the whole IS phase space is TI except for the point $\lambda_{\mathrm{so}}=0$. Thus the four-band model for graphene predicts that an IS trilayer will be either metallic (gapless) or a topological insulator and that a normal insulting region can only be accessed by breaking the inversion symmetry.

Illustrative $\lambda_{m}-\lambda_{\text {so }}$ cross sections of the four dimensional phase space of Hamiltonian (4.15) are shown in Fig. 4.6. The Hamiltonian (4.19) describing the phase space of a RS trilayer contains three parameters, $\lambda_{R}$ being zero. The corresponding $\lambda_{m}-\lambda_{\text {so }}$ cross sections are shown in Figs. 4.6(a) and 4.6(c). An interesting point in Fig. 4.6(c) is that the system has a metallic phase if $\lambda_{B}$ dominates $\lambda_{\mathrm{so}}$ and $\lambda_{m}$. In the Results Section we will see that this is the case for the $\mathrm{RS} \mathrm{MX}_{2}|\overline{\mathrm{C}}| \mathrm{MX}_{2}$ systems we study. However as can be seen in the other panels, when $\lambda_{B}$ or $\lambda_{R}$ is zero, the metallic region disappears.

Another interesting point can be seen in panels (b) and (d). Although the gap vanishes on the vertical lines in the two panels, the Pfaffian is not zero on either side of it and they can be connected to the $\lambda_{m} \rightarrow \infty$ or $\lambda_{m} \rightarrow-\infty$ points. Thus although the two regions of NI are separated by a vertical gapless line, the topological invariant does not change across it and therefore both regions are NI. For AS systems all four panels in Fig. 4.6 can occur. However we found that panel (d) describes the part of phase space relevant for our AS $\overline{\mathrm{C}} \mid \mathrm{MX}_{2}$ bilayers for which $\lambda_{B}$ and $\lambda_{R}$ are dominant.

\subsection{Results}

Calculations for each graphene- $\mathrm{MX}_{2}$ system were carried out for three configurations, the asymmetric (AS) bilayer and reflection symmetric (RS) and inversion symmetric (IS) trilayers to trace the effect of different symmetries on the SOI induced by $\mathrm{MX}_{2}$ layer(s).

\subsubsection{AS parameters and band structure}

The Hamiltonian describing asymmetrical bilayers is $H_{\mathbf{K}}^{\mathrm{AS}}(\mathbf{q})$ in (4.15). Since it contains four parameters $\left(\lambda_{m}, \lambda_{\mathrm{so}}, \lambda_{R}, \lambda_{B}\right)$ and there are four bands at the $\mathbf{K}$ point with the constraint that $\operatorname{Tr}\left[H_{\mathbf{K}}^{\mathrm{AS}}(\mathbf{q})\right]=0$, our system of equations is underdetermined. To solve this problem we use the expectation values of $s_{z}$ calculated with the first-principles wavefunction of the system together with the eigenvalues and slopes of the bands at the $\mathbf{K}$ point to generate the bands, their derivatives, and expectation values of $s_{z}$, for all $\mathbf{q}$. The first-principles pseudo wavefunction has the form $\tilde{\psi}_{n \mathbf{k}}(\mathbf{r})=\left(\tilde{\psi}_{n \mathbf{k}}^{\uparrow}(\mathbf{r}), \tilde{\psi}_{n \mathbf{k}}^{\downarrow}(\mathbf{r})\right)$, where each spin part is expanded in a plane wave basis as $\tilde{\psi}_{n \mathbf{k}}^{\sigma}(\mathbf{r})=\sum_{\mathbf{g}_{i}} C_{n \mathbf{k}}^{\sigma \mathbf{g}_{i}} e^{i\left(\mathbf{k}+\mathbf{g}_{i}\right) \cdot \mathbf{r}}$ where $\sigma$ is the spin component of the wave function and the summation is over reciprocal lattice vectors $\mathbf{g}_{i}$. The projection operator is $s_{z}$ 
so we have

$$
\left\langle\tilde{\psi}_{m \mathbf{K}}\left|s_{z}\right| \tilde{\psi}_{n \mathbf{K}}\right\rangle=\sum_{\mathbf{g}_{\mathbf{i}}}\left[\left(C_{m \mathbf{K}}^{\uparrow \mathbf{g}_{i}}\right)^{*} C_{n \mathbf{K}}^{\uparrow \mathbf{g}_{i}}-\left(C_{m \mathbf{K}}^{\downarrow \mathbf{g}_{i}}\right)^{*} C_{n \mathbf{K}}^{\downarrow \mathbf{g}_{i}}\right]
$$

Since $\tilde{\psi}_{n \mathbf{K}}$ are not orthonormalized, we transform these matrix elements to an orthonormal basis to calculate the expectation values. Since the trace of $s_{z}$ is zero we can only obtain three independent equations from using $\left\langle s_{z}\right\rangle_{n \mathbf{K}}$ giving us six equations at the $\mathbf{K}$ point. We can therefore determine up to six parameters from the $\mathbf{K}$ point only. However, we only need four parameters so our system of equations is now overdetermined. We can use this to test the consistency of the effective Hamiltonian. To determine the parameters we calculate the expectation value of $s_{z}$ which yields:

$$
\left\langle s_{z}\right\rangle_{\mathbf{K}}=(s, 1,-1,-s)
$$

where

$$
s=\frac{\left|\lambda_{B}-\lambda_{m}\right|}{\sqrt{\left(\lambda_{B}-\lambda_{m}\right)^{2}+\lambda_{R}^{2}}}
$$

and the order in 4.27 corresponds to the first-principles results for $A S \bar{C} \mid M_{2}$. The expectation values of $s_{x}$ can be written as in (4.26) as

$$
\left\langle\tilde{\psi}_{m \mathbf{K}}\left|s_{x}\right| \tilde{\psi}_{n \mathbf{K}}\right\rangle=\sum_{\mathbf{g}_{i}}\left[\left(C_{m \mathbf{K}}^{\uparrow \mathbf{g}_{i}}\right)^{*} C_{n \mathbf{K}}^{\downarrow \mathbf{g}_{i}}+\left(C_{m \mathbf{K}}^{\downarrow \mathbf{g}_{i}}\right)^{*} C_{n \mathbf{K}}^{\uparrow \mathbf{g}_{i}}\right]
$$

For Hamiltonian (4.15) with large $\lambda_{R}$, we found $\left\langle s_{x(y)}\right\rangle_{\mathbf{K}}=(0,0,0,0)$ for all AS systems that we studied. $\left\langle s_{x}\right\rangle_{\mathbf{K}}$ and $\left\langle s_{z}\right\rangle_{\mathbf{K}}$ are in agreement with results from (4.15) and therefore the four parameters are enough to describe AS $\bar{C} \mid \mathrm{MX}_{2}$. For example the $\left\langle s_{z}\right\rangle_{\mathbf{K}}$ for AS $\overline{\mathrm{C}} \mid \mathrm{WTe} \mathrm{T}_{2}$ are $\left\langle s_{z}\right\rangle_{\mathbf{K}}=(0.45,1.00,-1.00,-0.45)$ and $\left\langle s_{x}\right\rangle_{\mathbf{K}}=$ $(0.00,0.00,0.00,0.00)$. The parameters $\lambda_{m}, \lambda_{\mathrm{so}}, \lambda_{R}, \lambda_{B}$ could then be found from the eigenvalues at the $\mathbf{K}$ point and $\left\langle s_{z}\right\rangle_{\mathbf{K}}$ and the ordering in (4.16) is a result of order in $\left\langle s_{z}\right\rangle_{\mathbf{K}}$. If we set $\left\langle s_{z}\right\rangle_{\mathbf{K}}=(s, 1,-1,-s)$, then the parameters are:

$$
\begin{aligned}
& \lambda_{\mathrm{so}}=\left(\varepsilon_{2}+\varepsilon_{3}-\varepsilon_{1}-\varepsilon_{4}\right) / 4 \\
& \lambda_{m}=\left(\varepsilon_{2}+s \varepsilon_{4}-\varepsilon_{3}-s \varepsilon_{1}\right) / 4 \\
& \lambda_{B}=\left(\varepsilon_{2}-s \varepsilon_{4}-\varepsilon_{3}+s \varepsilon_{1}\right) / 4 \\
& \lambda_{R}= \pm\left(\varepsilon_{1}-\varepsilon_{4}\right) \sqrt{1-s^{2}} / 2
\end{aligned}
$$

By determining the four parameters in the phenomenological Hamiltonian (4.15) in this way we can describe the band structure, derivatives of bands, and spinexpectation values for all low-energy bands essentially perfectly. The results of doing this for all $\mathrm{AS} \overline{\mathrm{C}} \mid \mathrm{MX}_{2}$ bilayers are given in Table 4.2.

Another point worth mentioning is that if supercells are used for which $N_{\overline{\mathrm{C}}}$ is a multiple of 3 , then both $\mathbf{K}$ and $\mathbf{K}^{\prime}$ map onto the $\boldsymbol{\Gamma}$ point of the supercell. Since the spin direction of bands changes from $\mathbf{K}$ to $\mathbf{K}^{\prime}$, then at $\mathbf{K}^{\prime}$ we have $\left\langle s_{z}\right\rangle_{\mathbf{K}^{\prime}}=$ $(-s,-1,1, s)$. Because the bands from $\mathbf{K}$ are degenerate with the corresponding 


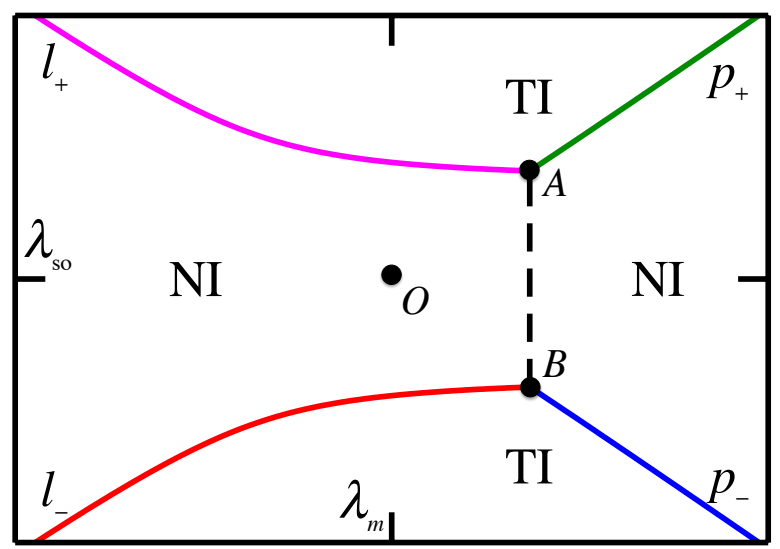

Figure 4.7: Phase space for an $\mathrm{AS} \overline{\mathrm{C}} \mid \mathrm{MX}$ bilayer with threefold rotational symmetry. The $x$ axis is $\lambda_{m}$, the $y$ axis is $\lambda_{\mathrm{so}}$. The ab-initio calculated parameter values $\lambda_{m}^{\mathrm{AS}}$ and $\lambda_{\mathrm{so}}^{\mathrm{AS}}$ are given in Table 4.2.

bands from $\mathbf{K}^{\prime}$, the first-principles wavefunction will now be determined up to a $U(2)$ transformation of these two wavefunctions. The wavefunction with $\left\langle s_{z}\right\rangle=s$ mapped from $\mathbf{K}$ will be degenerate with the wavefunction from $\mathbf{K}^{\prime}$ with $\left\langle s_{z}\right\rangle=-s$, and the space these two functions span will be a linear combination of the two. For any arbitrary $\left\langle s_{z}\right\rangle$, any value between -1 and 1 is possible. Under these circumstances using $\left\langle s_{z}\right\rangle$ values of the wave functions to find the parameters becomes meaningless and additional information must be used.

To determine the topological invariants for AS bilayers, we now turn to the phase space of the Hamiltonian (4.15) for which different cross sections are illustrated in Fig. 4.6. From Table 4.2 we see that for the six bilayers we have studied $\left|\lambda_{R}\right| \sim$ $\left|\lambda_{B}\right|>>\left|\lambda_{\mathrm{so}}\right| \sim\left|\lambda_{m}\right|$ making Fig. 4.6(d) the relevant cross section (but noting that all $\lambda_{B}$ in the Table are negative). Because of the small magnitudes of $\lambda_{\text {so }}$ and $\lambda_{m}$, all bilayers are very close to the origin in the phase space shown in Fig. 4.7 where TI

Table 4.2: Calculated gaps and parameters in meV for equilibrium $\mathrm{AS} \overline{\mathrm{C}} \mid \mathrm{MX}{ }_{2}$ configurations.

\begin{tabular}{lccccccc}
\hline $\mathrm{MX}_{2}$ & $s$ & $\Delta_{g}$ & $\Delta_{K}$ & $\lambda_{m}$ & $\lambda_{\text {so }}$ & $\lambda_{R}$ & $\lambda_{B}$ \\
\hline $\mathrm{MoS}_{2}$ & 0.91 & 0.12 & 0.55 & -0.08 & 0.00 & 0.12 & -0.27 \\
$\mathrm{MoSe}_{2}$ & 0.74 & 0.45 & 1.01 & 0.05 & 0.00 & 0.54 & -0.55 \\
$\mathrm{MoTe}_{2}$ & 0.52 & 0.77 & 1.24 & -0.02 & 0.01 & 0.96 & -0.60 \\
$\mathrm{WS}_{2}$ & 0.95 & 0.25 & 1.58 & -0.01 & 0.00 & 0.25 & -0.78 \\
$\mathrm{WSe}_{2}$ & 0.48 & 0.92 & 1.42 & -0.05 & 0.00 & 1.11 & -0.66 \\
$\mathrm{WTe}_{2}$ & 0.45 & 1.38 & 1.96 & -0.01 & 0.00 & 1.92 & -0.98 \\
\hline
\end{tabular}



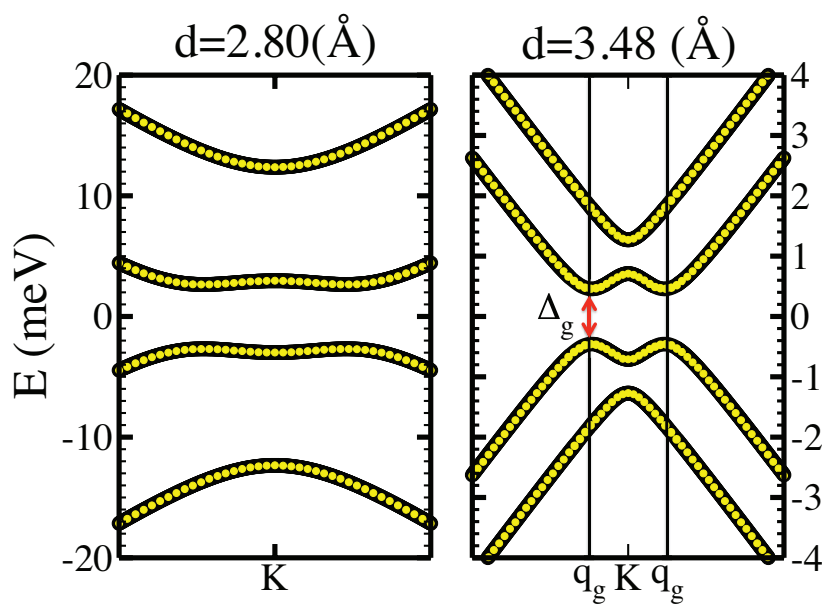

Figure 4.8: Band structures of $\mathrm{AS} \overline{\mathrm{C}} \mid \mathrm{WSe}_{2}$ at $d=2.80 \AA$ (left) and $d_{\mathrm{eq}}=3.48 \AA$ (right). The band structure under pressure becomes flatter because $\lambda_{R}$ increases faster than $\lambda_{B}$.

regions are separated from NI regions by the boundaries $l_{ \pm}$given by solving

$$
\lambda_{B}+\lambda_{m} \pm 2 \lambda_{\text {so }}-\sqrt{\left(\lambda_{B}-\lambda_{m}\right)^{2}+\lambda_{R}^{2}}=0
$$

and $p_{ \pm}$given by solving

$$
\lambda_{B}+\lambda_{m} \mp 2 \lambda_{\text {so }}+\sqrt{\left(\lambda_{B}-\lambda_{m}\right)^{2}+\lambda_{R}^{2}}=0 .
$$

The Pfaffian is zero in the region labelled TI and non zero in the regions labelled NI. On the vertical black dashed line $\lambda_{B}+\lambda_{m}=0$, the system is gapless but the Pfaffian does not change to zero and therefore the topological invariant will remain unchanged. From Fig. 4.7 and the parameters in Table 4.2 we expect all AS $\bar{C} \mid \mathrm{MX}_{2}$ bilayers to be normal insulators and therefore the $\mathbb{Z}_{2}$ invariant to be zero.

To study the effect of uniaxial pressure, we calculated the electronic structure of AS $\bar{C} \mid W_{2} e_{2}$ self consistently as a function of the interlayer separation $d$ to determine the $\lambda$ parameters. We show typical low energy band structures close to the $\mathbf{K}$ point for the equilibrium separation $d_{\mathrm{eq}}=3.48 \AA$ and for a small separation $d=2.80 \AA$. The bands are seen to become "flatter" because the Rashba term increases under pressure faster than $\lambda_{B}$. The minimum gap is seen to occur a distance $q_{g}$ from the origin where $q_{g}$ is given by (4.17) and the corresponding gap $\Delta_{g}$ is given by (4.18). The pressure corresponding to a certain interlayer separation $d$ can be estimated by differentiating the total energy of the system with respect to $d$ and dividing by the supercell area $\Omega, P=-(1 / \Omega) \partial E / \partial d$. The dependence of the gap on $d$ (pressure) is shown in Fig. 4.9, and in Fig. 4.10 we show the two dominant parameters $\lambda_{R}$ and $\lambda_{B}$ 


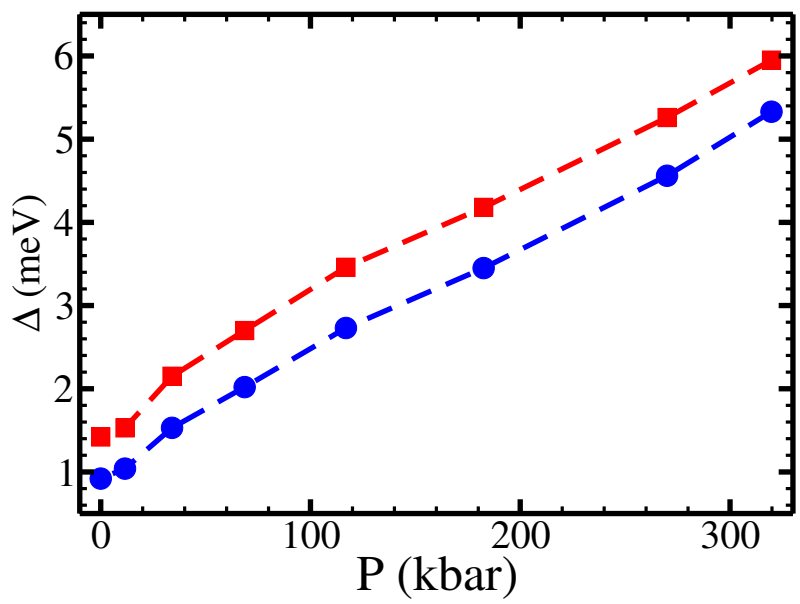

Figure 4.9: Band gaps at the $\mathbf{K}$ point (red squares and line) and at the $q_{g}$ point (blue circles and line) as a function of pressure for $\mathrm{AS} \overline{\mathrm{C}} \mid \mathrm{WSe}_{2}$.

as a function of the interlayer separation. As can be seen from Fig. 4.10(a), the Rashba term has a larger slope than the internal magnetic term $\lambda_{B}$, and from the phase space we conclude that applying pressure to this bilayer will not change its topological invariant and the system will remain a normal insulator. The external pressure has effects on the $\mathrm{MX}_{2}$ band structures too. In Fig. 4.10(b) we show $\lambda_{o}(d)$ the separation of the Dirac cone's center of graphene from the top of the valence band of $\mathrm{MX}_{2}$ as a function of separation $d$. Under pressure the valence band of $\mathrm{MX}_{2}$ moves upward toward the graphene Dirac bands and for interlayer separations smaller than $2.8 \AA$ the valence band of $\mathrm{MX}_{2}$ and graphene $p_{z}$ bands at the $\mathbf{K}$ point will cross. When this happens, our model which is based on four $p_{z}$ bands of graphene will no longer be applicable.

\subsubsection{RS parameters}

For an $\mathrm{MX}_{2}|\overline{\mathrm{C}}| \mathrm{MX}_{2}$ trilayer with reflection symmetry $\lambda_{R}$ should vanish since it is odd under reflection leaving us to determine three parameters from equations (4.20) that are given in Table 4.3. With $\lambda_{R}=0$ the effective Hamiltonian (4.19) is diagonal in spin so $\left\langle s_{z}\right\rangle= \pm 1$. This is in good agreement with the results of the first-principles calculations where the expectation values of $s_{z}$ for the four low energy bands at the $\mathbf{K}$ point are $\left\langle s_{z}\right\rangle_{\mathbf{K}}=(1,1,-1,-1)$.

In Table $4.3, \lambda_{B}$ is seen to be the dominant parameter. The effective Hamiltonian $H_{\mathbf{K}}^{\mathrm{RS}}$ in (4.19) predicts zero gap for reflection symmetric structures. This is in good agreement with the tiny gaps $\Delta_{g}$ given in Table 4.3 that were calculated from firstprinciples. These first-principles gaps are an order of magnitude smaller than those calculated for the AS structure (Table 4.2) and do not show a strong dependence on 

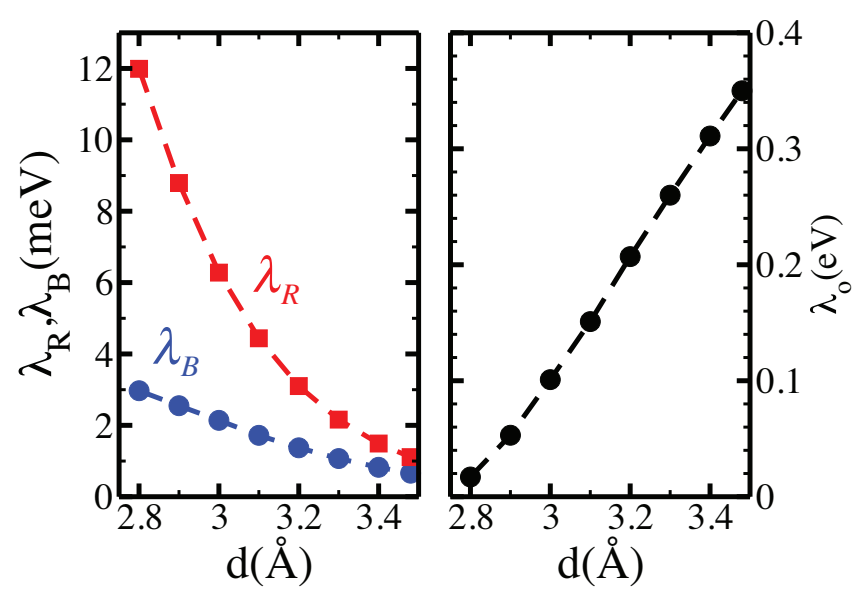

Figure 4.10: (a): Dependence of $\lambda_{R}$ (squares, red line) and $\lambda_{B}$ (circles, blue line) on the separation $d$ of $\overline{\mathrm{C}}$ from $\mathrm{WSe}_{2}$ for an $\mathrm{AS} \overline{\mathrm{C}} \mid \mathrm{WSe} \mathrm{e}_{2}$ bilayer. The equilibrium separation is $3.48 \AA$ and $\lambda_{R}$ is dominant over the whole range. (b): $\lambda_{o}$, the separation of $\varepsilon_{1}$ from the top of the valence band of $\mathrm{WSe}_{2}$.

Table 4.3: Calculated gaps and parameters in meV for $\mathrm{RS}^{\mathrm{MX}} \mathrm{X}_{2}|\overline{\mathrm{C}}| \mathrm{MX}_{2}$ trilayers.

\begin{tabular}{lccccc}
\hline & $\Delta_{g}$ & $\Delta_{\mathbf{K}}$ & $\lambda_{m}$ & $\lambda_{\text {so }}$ & $\lambda_{B}$ \\
\hline $\mathrm{MoS}_{2}$ & 0.04 & 0.99 & 0.00 & 0.02 & 0.51 \\
$\mathrm{MoSe}_{2}$ & 0.03 & 1.91 & 0.00 & 0.10 & 1.06 \\
$\mathrm{MoTe}_{2}$ & 0.07 & 3.31 & 0.01 & 0.01 & 1.67 \\
$\mathrm{WS}_{2}$ & 0.03 & 2.93 & 0.00 & 0.03 & 1.50 \\
$\mathrm{WSe}_{2}$ & 0.09 & 2.21 & 0.00 & 0.01 & 1.11 \\
$\mathrm{WTe}_{2}$ & 0.00 & 4.66 & 0.00 & 0.01 & 2.34 \\
\hline
\end{tabular}

the $\mathrm{X}$ atom or $\mathrm{M}$ atom. In addition, they are an upper bound since a more refined search on a finer k-mesh could lead to lower values. Because the vanishing of the off-diagonal terms in our effective Hamiltonian is a consequence of the trilayer reflection symmetry, we expect the metallic phase of this system to be robust against translational transformations of the unit cell. However, such translations can generate additional terms (the $\mathbf{f}_{3}$ and $\mathbf{f}_{4}$ terms discussed in the Appendix) from the $\mathbf{f}_{\mathbf{K}} \cdot \boldsymbol{\sigma}$ term in (4.11) that have been neglected here. Including these could lead to the RS trilayer becoming a TI with very small gaps.

\subsubsection{IS parameters and band structure}

For an $\mathrm{MX}_{2}|\overline{\mathrm{C}}| \mathrm{MX}_{2}$ trilayer with inversion symmetry, $\lambda_{R}$ is predicted to be globally zero. The $\lambda_{m}$ mass term and the $\lambda_{B}$ pseudomagnetic term are rigorously zero since they are odd under inversion. Besides the Fermi velocity term, the effective Hamilto- 
nian $H_{\mathrm{K}}^{\mathrm{IS}}$ in (4.22) contains only the $\lambda_{\mathrm{so}}$ term. Since the bands are paired and the two bands of each pair have opposite spin texture, $\left\langle s_{z}\right\rangle$ cannot be determined. However, since there is only one parameter left to be determined, there is no need to know the spin expectation values. The $H_{\mathbf{K}}^{\mathrm{IS}}$ model predicts a non-zero gap for IS systems. For all IS $\mathrm{MX}_{2}|\overline{\mathrm{C}}| \mathrm{MX}_{2}$ structures we found that the minimum gaps are at the $\mathbf{K}$ point and they are very small (smaller than $0.1 \mathrm{meV}$ ) in agreement with the results for the AS bilayer. From the phase space arguments given in Section 4.3.4, we know that the IS systems are topological insulators. The gaps could be increased by breaking the $C_{3}$ rotational symmetry but the topological non-triviality of the IS systems will not change. Applying external pressure will also not change the character of the IS trilayers.

We can use the formula given by Fu and Kane [32]

$$
(-1)^{\nu}=\prod_{i} \delta_{i}
$$

for inversion symmetric systems to determine the topological invariant $\nu$ explicitly from the wave functions in first-principles calculations. In (4.33) the multiplication is over all the time-reversal fixed points in the Brillouin zone $\left(\Gamma_{i}\right)$, and

$$
\delta_{i}=\prod_{m=1}^{N} \xi_{2 m}\left(\Gamma_{i}\right)
$$

where the multiplication is over bands with even band number at the $\Gamma_{i}$ and $\xi_{2 m}$ is the parity eigenvalue of the bands $2 m-1$ and $2 m$. In a first-principles calculation for a large supercell with many bands, practical complications can occur. The energy separations of non-degenerate bands might be comparable with the numerical accuracy of bands that should be degenerate, or there can be higher order degeneracies due to extra crystal symmetries that are compatible with inversion and time reversal symmetries. Each pair of Kramers degenerate bands (degenerate by time reversal and inversion symmetry) will have the same parity eigenvalue, but if there is fourfold degeneracy (or close to degeneracy) then the multiplication of parity eigenvalues will be ambiguous if we only take into account the bands with even (odd) band number. To avoid such difficulties we take all the degenerate (to within some tolerance) bands into account. Generally, there are $2 N$ occupied bands, $r$ pairs of which have negative parity eigenvalue and the rest have positive eigenvalues. If $\xi_{m}$ is now the parity eigenvalue of band $m$ then the number of odd pairs will be

$$
r=\frac{2 N-\sum_{m=1}^{2 N} \xi_{m}}{4}
$$

$(-1)^{\delta_{i}}=(-1)^{r}$ and there is no ambiguity in the final result. Using this procedure, trilayer structures with inversion and three-fold symmetry were found to be topological insulators with small band gaps. 

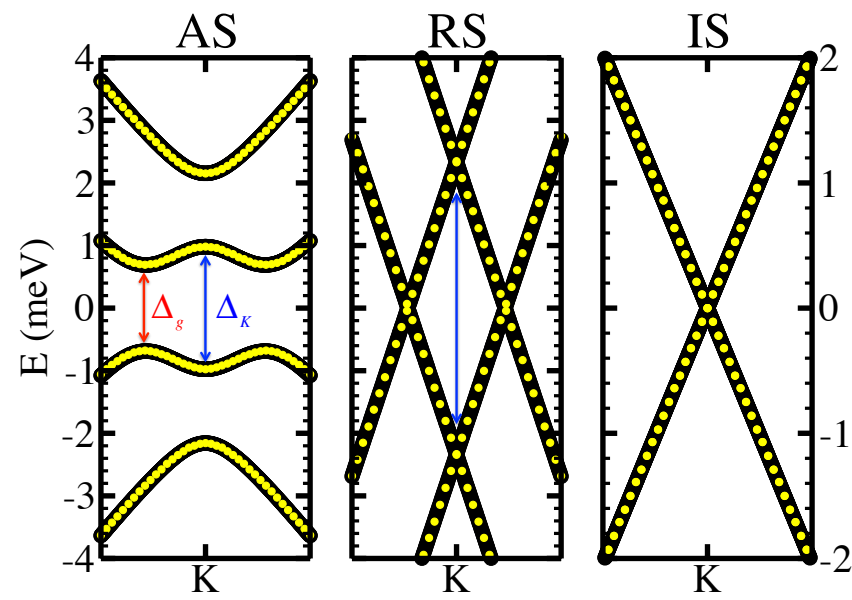

Figure 4.11: Band structures of graphene on $\mathrm{WTe}_{2}$ close to the $\mathbf{K}$ point for different configurations. The yellow circles are the results of first principles calculations, the black lines are the effective Hamiltonian bands.

\subsection{Conclusion}

We have explored the spin-orbit splitting induced in graphene by interaction with transition metal dichalcogenides and found that inversion and reflection symmetries are important for the general features of the graphene band structure close to the $\mathbf{K}$ point. This is very clearly seen in Fig. 4.11 which shows the band structures for AS, RS, and IS graphene on $\mathrm{WTe}_{2}$ calculated from first-principles (yellow dots) and using a low-energy effective Hamiltonian (black lines) developed in Section 4.3 by downfolding a tight-binding model. The model describes the bands close to the $\mathbf{K}$ points very accurately. By combining our knowledge of the first-principles topological invariants with the predictions of the effective Hamiltonian model, we could conclude that the non-trivial invariant is robust against relative translations of the layers and pressure.

Compared to the model Hamiltonian introduced by Kane and Mele [29, 30], our effective Hamiltonian contains five additional terms. The first of these, $\lambda_{B} \sigma_{o} \tau_{z} s_{z}$, has the form of a pseudo internal magnetic field and is characterized by the parameter $\lambda_{B}$. The other four terms depend on eight parameters $\mathbf{f}_{1}, \mathbf{f}_{2}, \mathbf{f}_{3}$, and $\mathbf{f}_{4}$ (discussed in the Appendix) which respect the time reversal symmetry and are therefore allowed by symmetry. We constructed low energy Hamiltonians for AS, RS, and IS systems. A systematic method to find the parameters from the eigenvalues and vectors at the $\mathbf{K}$ point was developed and the results found to be in good agreement with the results of first-principles calculations.

We established arguments to allow the $\mathbb{Z}_{2}$ invariant to be calculated from lowenergy states. Based on phase space analyses, we concluded that with SOC-induced 
gaps less than $1 \mathrm{meV}$ at the equilibrium separations, $\mathrm{AS} \overline{\mathrm{C}} \mid \mathrm{MX} \mathrm{X}_{2}$ bilayers are not $\mathbb{Z}_{2}$ topological insulators. The same procedure was applied to RS structures which we showed to be either metallic or TI for $C_{3}$ symmetric supercells. By calculating the Pfaffian from first-principles we moreover demonstrated that IS $\mathrm{MX}_{2}|\overline{\mathrm{C}}| \mathrm{MX}_{2}$ trilayers are $\mathbb{Z}_{2}$ topological insulators but with very small band gaps at their equilibrium separations for $C_{3}$ symmetric cells.

The band gap is reduced significantly for RS trilayers by the absence of a Rashba $\lambda_{R}$ term and the presence of an internal pseudo magnetic $\lambda_{B}$ term which dominates the "intrinsic" $\lambda_{\text {so }}$ term. The IS trilayers also have very small band gaps because $\lambda_{\text {so }}$ is so small and other terms that could open a gap are not allowed by symmetry. The AS bilayer was realised by putting graphene in contact with a single layer of $\mathrm{MX}_{2}$ to break the inversion and reflection symmetry of graphene plane. The resulting band gap is dominated by the spin-orbit interaction and is about $1 \mathrm{meV}$ at the equilibrium separation for $\mathrm{WSe}_{2}$ and $\mathrm{WTe}_{2}$ (Fig. 4.11). On applying uniaxial pressure, we observed that the band gap increases due to the increase in the hybridization between layers which results in an increase in the induced spin-orbit parameters. However, the topological invariant did not change.

\subsection{Appendix: non-zero $f_{K}$}

In deriving (4.15) we neglected $\mathbf{f}_{\mathrm{K}}$ in (4.11). For an arbitrary substrate however, $f_{K}$ does not vanish and must be taken into consideration. If the substrate does not have three-fold symmetry or if the interacting orbitals of the substrate have non-zero interactions with each other at the $\mathbf{K}$ point, then it becomes necessary to include $\mathbf{f}_{\mathbf{K}}$. For example, in $\mathrm{MX}_{2}$ the hopping between the $\mathrm{M}$ atom $d_{3 z^{2}-r^{2}}$ orbitals and $\mathrm{X}$ atom $p_{z}$ orbitals is not zero at the $\mathbf{K}$ and $\boldsymbol{\Gamma}$ points. The correction to (4.15) resulting from $f_{K}$ is

$$
H_{f}=\mathbf{f}_{1} \cdot \boldsymbol{\sigma}+\mathbf{f}_{2} \cdot \boldsymbol{\sigma} s_{z}+\mathbf{f}_{3} \cdot \mathbf{s} \sigma_{z}+\mathbf{f}_{4} \cdot \mathbf{s}
$$

where the $\mathbf{f}_{i}=\mathfrak{R e} f_{i}(\mathbf{K}) \hat{x}+\mathfrak{I m} f_{i}(\mathbf{K}) \hat{y}$ are vectors and $H_{f}$ should be added to the Hamiltonian (4.15). All four $\mathbf{f}_{i}$ vectors depend on $\left|\mathbf{f}_{\mathbf{K}}\right|$ and vanish if it is zero. However they appear in the final Hamiltonian mixed with different Slater-Koster parameters of different orbitals and are not simply related. We obtained the $\mathbf{f}$ terms by breaking three-fold symmetry in the substrate $\left(\mathbf{f}_{\mathbf{K}} \neq 0\right)$. However, the $C_{3}$ symmetry can be broken by interactions between graphene and a substrate and on general grounds, we expect that this type of interaction can also be represented by $\mathbf{f}$ terms. The supercells used in this work all have $C_{3}$ rotational symmetry around the inversion-center so that the $\mathrm{f}$ terms should be minuscule. This is why the parameterization neglecting $H_{f}$ in (4.36) could describe the band structures and spin expectation values so accurately.

The first term in (4.36), $\mathbf{f}_{1} . \boldsymbol{\sigma}$, will shift the Dirac point away from the $\mathbf{K}$ point meaning that it will break the direction-independent character of the bands around the $\mathbf{K}$ point. The bands will still be symmetric around the point $\mathbf{q}=-\mathbf{f}_{1} / \hbar v_{F}$ and 
therefore this term alone will not open a band gap. The second term, $\mathbf{f}_{2} . \boldsymbol{\sigma} s_{z}$, will also shift the Dirac point away from the $\mathbf{K}$ point, however the shift is opposite for opposite spins. It also does not open a gap by itself. These two terms will change the symmetrical shape of the bands about the $\mathbf{K}$ point and therefore can be detected as a direction dependence with respect to $\mathbf{q}$.

The third term, $\mathbf{f}_{3} . \mathbf{s} \sigma_{z}$, is the $(x, y)$ component of the $\lambda_{\text {so }}$ term since it is even under time-reversal and inversion like $\lambda_{\text {so }}$ and similar to it in form. The fourth term, $\mathbf{f}_{4} . \mathbf{s}$, is the $(x, y)$ component of the pseudomagnetic field $\left(\lambda_{B}\right)$ because they are both odd under inversion and have a similar form.

The presence of the eight $\mathbf{f}$ terms in the Hamiltonian will change the band structure and will also increase the dimension of phase space to twelve $(4+8)$. We show one cross section of phase space with four different $f$ terms in Fig. 4.12. We have neglected the $\mathbf{f}_{1}$ and $\mathbf{f}_{2}$ terms for the sake of simplicity. In panel (a) we show the phases without $f$ term for reference. In the other panels we show three different ways that the $f$ terms can change this cross section. It can be seen that the M(etallic) regions in panel (a) become TI on introducing $f$ terms. This indicates that for the RS systems with threefold rotation symmetry for which we found no gap, introduction of $C_{3}$ breaking displacement of the layers will open a non-trivial gap.

$H_{\mathbf{K}}^{\mathrm{AS}}(\mathbf{q})+H_{f}$ contains a total of 12 parameters: $\lambda_{R}, \lambda_{B}, \lambda_{\mathrm{so}}, \lambda_{m}$ and the $8 \mathbf{f}_{i}$ parameters just discussed. The most general four-band Hamiltonian for graphene can have four more terms. In addition to a $\lambda_{o}$ term that only rigidly shifts the center of the graphene bands in energy that will be studied in the results section, these three terms can be represented as $\lambda_{x} \sigma_{x} s_{x}+\lambda_{y} \sigma_{y} s_{y}+\lambda_{+}\left(\sigma_{x} s_{y}+\sigma_{y} s_{x}\right)$. On constructing the full $32 \times 32 s p^{3}$ tight binding Hamiltonian for graphene on $h$-BN and downfolding it at $\mathbf{K}$, we did not observe these terms. We conclude that they do not represent any new symmetry and therefore they can be negligible and Rashba term is enough to describe our graphene on $\mathrm{MX}_{2}$ bilayers and trilayers. 


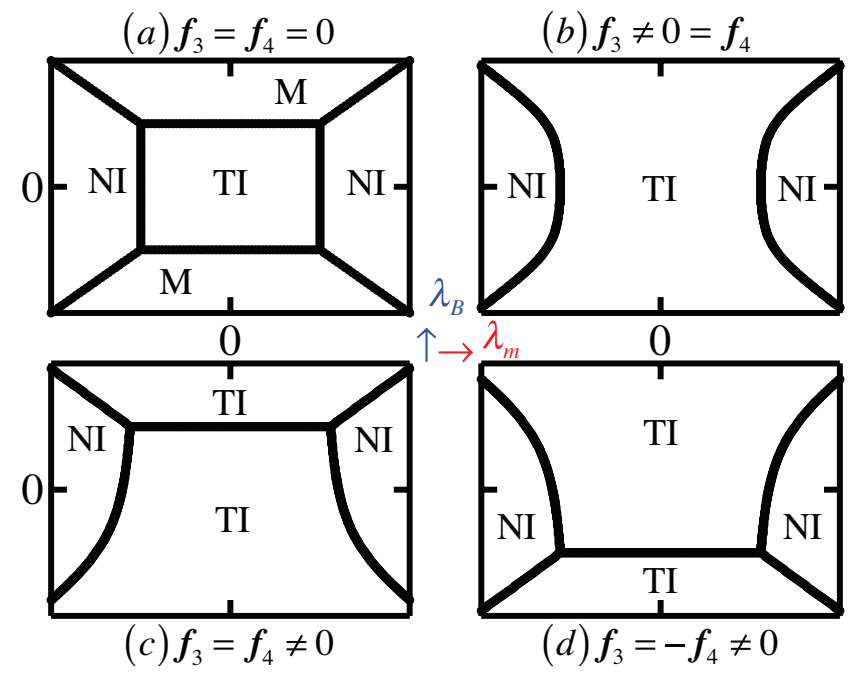

Figure 4.12: Phase space for reflection symmetric (RS) systems. The boundaries are determined by $\varepsilon_{3}-\varepsilon_{2}=0$. In panel (a) system is in metallic (M) phase, however if a gap opens in the system without breaking reflections symmetry then the system becomes TI. 


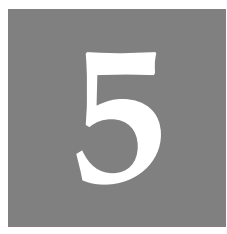

\section{Inducing spin-orbit gap in graphene on heavy metal compounds}

Graphene was the first material proposed to be a $\mathbb{Z}_{2}$ topological insulator but its minuscule spin-orbit coupling (SOC) makes experimental observation unlikely. We use density functional theory calculations to explore the possibility of inducing a larger SOC in graphene by letting it hybridize with the layered $\mathrm{MX}$ substrates $\mathrm{M}=\mathrm{Sn}, \mathrm{Pb}$; $\mathrm{X}=\mathrm{O}$ ) which have the heavy metal on the outside of the layer. We show that an inversion symmetric $\mathrm{SnO} \mid$ graphene $\mid \mathrm{SnO}$ trilayer is a $\mathbb{Z}_{2}$ topological insulator with a band gap of $0.7 \mathrm{meV}$ but that a graphene|SnO bilayer is a normal insulator in its equilibrium configuration with a larger gap of $6.8 \mathrm{meV}$. The Dirac point of graphene does not fall in the band gap of $\mathrm{PbO}$. 


\subsection{Introduction}

Our understanding of the insulating phase of materials has been changed by the discovery of topological insulators $[26,118]$. The explanation of quantization in the quantum Hall effect in terms of topological invariants [36] popularized the concept of the topological nature of wavefunctions. Another topological invariant was discovered for systems with time-reversal symmetry $[29,30]$ where the invariant is called $\mathbb{Z}_{2}$. A spin-dependent conductivity and robust edge states were predicted for graphene, the first candidate proposed to exhibit non-trivial $\mathbb{Z}_{2}$ behaviour. When SOC is included, a gap is opened at the $\mathbf{K}$ point where the linear crossing of two spindegenerate bands in the absence of SOC gives rise to many of graphene's remarkable properties [38]. However the intrinsic SOC in graphene is so small that the splitting induced at the $\mathbf{K}$ point is predicted to be less than $0.05 \mathrm{meV}[86,87,88,89,90]$ making experimental observation extremely demanding if not impossible.

Proximity to a material with a large SOC has been suggested as a way to induce larger spin-orbit interactions in graphene and several attempts have been made to find suitable substrates $[94,96,99]$. Similarity of the lattice structure and experimental advances in growing semiconducting transition metal dichalcogenide (TMD) substrates has led to a number of theoretical $[95,96,98]$ and experimental [97] studies of graphene on TMD substrates in the hope of finding large SOC induced gaps and $\mathbb{Z}_{2}$ topological phase.

The $\mathrm{MX}_{2}$ transition metal dichalcoginide structure consists of trilayers of transition metals $(\mathrm{M}=\mathrm{Mo}$ or $\mathrm{W})$ sandwiched between and covalently bonded to layers of chalcogens $(\mathrm{X}=\mathrm{S}, \mathrm{Se}, \mathrm{Te})$; each trilayer is weakly bonded to the neighbouring trilayers. The semiconducting band gap is formed between a filled nonbonding metal band and an empty antibonding metal-chalcogen conduction band. These states are localized on the metal atoms and interact correspondingly weakly with a physisorbed graphene layer. In this work we investigate using the MX litharge structure $(\mathrm{M}=\mathrm{Sn}$ or $\mathrm{Pb} ; \mathrm{X}=\mathrm{O})$ as a substrate and study the effective interactions it induces in graphene. Litharge also has a trilayer structure but with the heavy metal forming the outer layers so that stronger hybridization with a physisorbed graphene might be expected. Since litharge has a tetragonal structure [23, 119], putting graphene on MX will break the three-fold symmetry of graphene and the two-fold symmetry of $\alpha-\mathrm{MX}$ so the resulting structure will not have any symmetry.

\subsection{Method}

To determine equilibrium structures of free-standing MX layers, we used density functional theory (DFT) calculations as implemented with a Projector Augmented Wave (PAW) basis [8, 9] in the Vienna Ab-initio Simulation Package (VASP) [58]. A plane-wave basis with a cut-off energy of $600 \mathrm{eV}$ was used and a $24 \times 24 \times 1 \mathrm{k}$-mesh were used for MX and $42 \times 42 \times 1$ for graphene. The k-mesh was chosen in such a way as to include all the high symmetry points of both $\mathrm{MX}$ and graphene. The electronic 


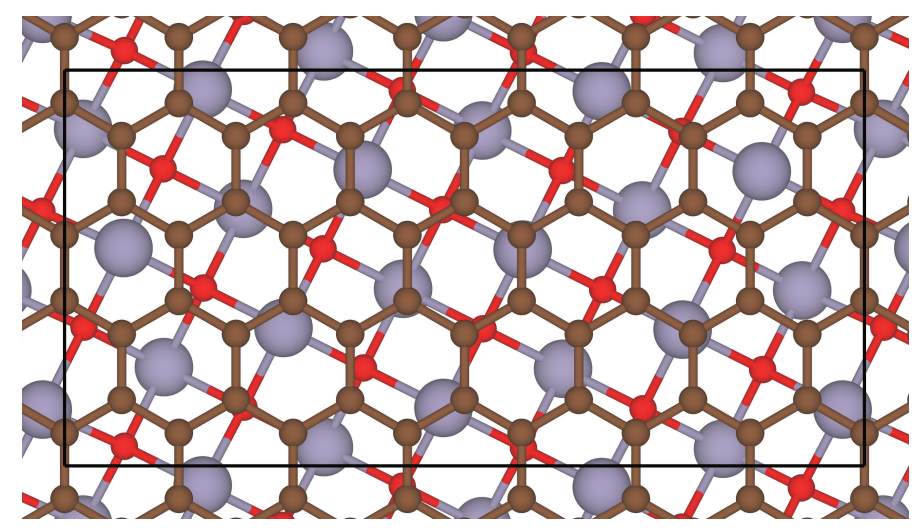

Figure 5.1: The unit cell of AS-C $\mid$ SnO corresponds to $\sqrt{49} \times \sqrt{12}$ graphene or $\sqrt{20} \times \sqrt{5}$ $\mathrm{SnO}$ unit cells. It contains 56 carbon (grey), 20 oxygen (red), and $20 \mathrm{Sn}$ (purple) atoms. The relative rotation between layers is $\theta=3.4^{\circ}$ and the strain is -0.4 and 0.6 percent respectively.

self-consistency criterion was set to $10^{-7} \mathrm{eV}$ during all of the calculations in order to obtain well converged band structures. Interaction between periodic images of the trilayer was minimized by separating them with a "vacuum layer". The vacuum thickness was set to $15 \AA$ and a dipole correction applied to avoid artificial effects of (long range) image charges [81].

As a compromise between the local density approximation (LDA) that tends to overbind, and the generalized gradient approximation (GGA) that underbinds van der Waals (vdW) structures, we used the optB88-vdW density functional (DF) [78, $102,103]$ that describes the weak bonding of graphene to substrates. In the vdW-DF, the exchange correlation functional is written as $E_{\mathrm{xc}}=E_{\mathrm{x}}+E_{\mathrm{c}}^{\mathrm{vdW}}+E_{\mathrm{c}}^{\mathrm{loc}}$, where $E_{\mathrm{c}}^{\mathrm{vdW}}$ describes non-local electron-electron correlations and $E_{\mathrm{c}}^{\text {loc }}$ local correlations. For the exchange part $E_{\mathrm{x}}$, we use the optB86 functional. This functional gives a satisfactory description of the lattice parameters and binding energy of graphene and MX.

We begin with a full geometry optimization of isolated monolayers of graphene $(\overline{\mathrm{C}})$ and $\mathrm{MX}$ to determine theoretical in-plane lattice constants. After this step we determine common $\overline{\mathrm{C}} \mid \mathrm{MX}$ supercells that accommodate both components with an acceptable amount of strain. The strain is then accommodated entirely in the MX "substrate" structure so that the graphene layer is in equilibrium. With the joint supercell lattice parameters now fixed, the MX structure is again relaxed. To construct $\overline{\mathrm{C}} \mid \mathrm{MX}$ unit cells with acceptable mismatch, we assume that the angle between graphene and MX layer can be chosen arbitrarily to make use of the rotational degree of freedom. Since the interaction between layers is weak, we expect that this rotation has no major effect on the electronic properties of graphene. Our experience with graphene on hexagonal boron nitride (Chapter 2) and graphene on transition metal dichalcogenides (Chapter 4) shows that small rotations of layers usually cause the 
(a)

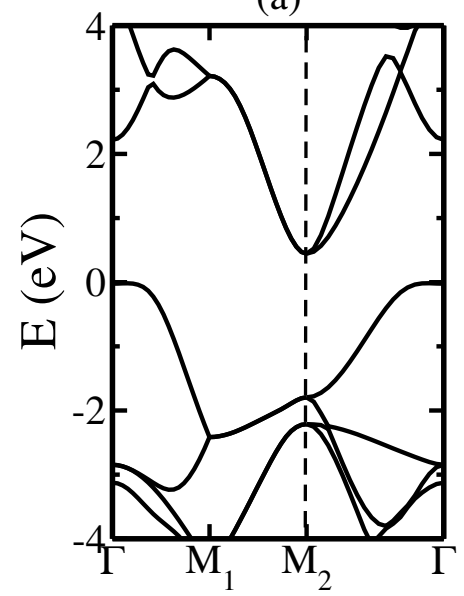

(b)

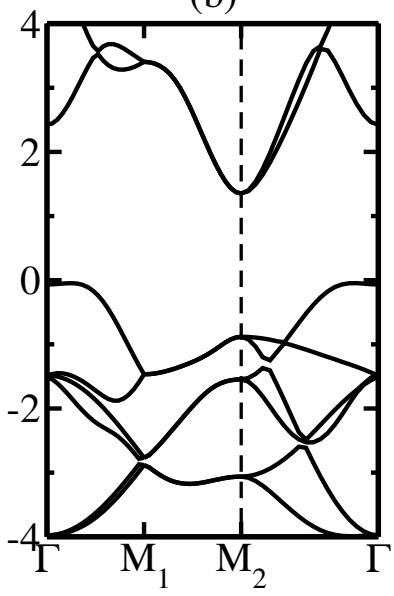

Figure 5.2: The band structure of (a) litharge-SnO (left) and (b) litharge- $\mathrm{PbO}$ (right). The band gap is indirect and about $0.4 \mathrm{eV}$ for $\mathrm{SnO}$ and $1.3 \mathrm{eV}$ for $\mathrm{PbO}$.

two layers to electronically decouple and decrease the gap found for aligned systems [18]. Because of the underestimation of gaps by the LDA, we expect that the gaps we find in this paper to be lower bound on the experimental results. We choose the two primitive lattice vectors of graphene to be $\mathbf{a}_{1(2)}=\frac{a}{2}(\sqrt{3}, \pm 1)$. Any lattice vector in the graphene layer with the basis $\left\{\mathbf{a}_{1}, \mathbf{a}_{2}\right\}$ can be expressed as $\mathbf{t}_{\overline{\mathrm{C}}}=n_{1} \mathbf{a}_{1}+n_{2} \mathbf{a}_{2}$ where $n_{1}$ and $n_{2}$ are integers, and in the MX layer with primitive lattice vectors $\mathbf{b}_{1}=b(1,0)$ and $\mathbf{b}_{2}=b(0,1)$ as $\mathbf{t}_{\mathrm{MX}}=m_{1} \mathbf{b}_{1}+m_{2} \mathbf{b}_{2}$ where $m_{1}$ and $m_{2}$ are integers.

To determine the supercell we start with one edge. We impose the condition that parallel to this edge

$$
\left|\mathbf{t}_{\overline{\mathrm{C}}}^{\|}-\mathbf{t}_{\mathrm{MX}}^{\|}\right| \leq \eta\left|\mathbf{t}_{\mathrm{MX}}^{\|}\right|
$$

where $\eta$ is some maximum acceptable value of the strain of the substrate. Solutions to this equation are a set of the four integers $\left(n_{1}, n_{2}, m_{1}, m_{2}\right)$ that we call $\left(n_{1}^{*}, n_{2}^{*} ; m_{1}^{*}, m_{2}^{*}\right)$. Before choosing one element of this set, we should find the size of the other vector of the supercell. For each vector $\mathbf{t}_{\overline{\mathrm{C}}}^{\|}=n_{1}^{*} \mathbf{a}_{1}+n_{2}^{*} \mathbf{a}_{2}$ of the graphene layer and $\mathbf{t}_{\mathrm{MX}}^{\|}=m_{1}^{*} \mathbf{b}_{1}+m_{2}^{*} \mathbf{b}_{2}$ of the MX layer, orthogonal vectors can be constructed as

$$
\begin{aligned}
& \mathbf{t}_{\overline{\mathrm{C}}}^{\perp}=\quad \beta\left[ \pm\left(n_{1}^{*}+2 n_{2}^{*}\right) \mathbf{a}_{1} \mp\left(2 n_{1}^{*}+n_{2}^{*}\right) \mathbf{a}_{2}\right] \\
& \mathbf{t}_{\mathrm{MX}}^{\perp}=\alpha\left[ \pm m_{2}^{*} \mathbf{b}_{1} \mp m_{1}^{*} \mathbf{b}_{2}\right]
\end{aligned}
$$

where $\alpha$ and $\beta$ should be chosen in such a way that the coefficients

$$
\beta\left(n_{1}^{*}+2 n_{2}^{*}\right) ; \beta\left(2 n_{1}^{*}+n_{2}^{*}\right) ; \alpha m_{1}^{*} ; \alpha m_{2}^{*}
$$


Table 5.1: The calculated parameters of graphene, SnO, and PbO. $a$ ( $\AA$ is the lattice constant, $c(\AA$ is the vertical separation of $\mathrm{X}-\mathrm{X}$ atoms, $W(\mathrm{eV})$ is the work function and $\Delta(\mathrm{eV})$ is the band gap.

\begin{tabular}{lrrrr}
\hline & $a$ & $c$ & $W$ & $\Delta$ \\
\hline $\mathrm{PbO}$ & 4.05 & 2.39 & 4.76 & 2.28 \\
$\mathrm{SnO}$ & 3.86 & 2.35 & 5.58 & 2.88 \\
$\mathrm{Gr}$ & 2.47 & - & 4.56 & - \\
\hline
\end{tabular}

are integers and $\mathbf{t}_{\overline{\mathrm{C}}} \frac{\perp}{\text { and }} \mathbf{t}_{\mathrm{MX}}^{\perp}$ satisfy the relation

$$
\left|\mathbf{t}_{\mathrm{C}}^{\perp}-\mathbf{t}_{\mathrm{MX}}^{\perp}\right| \leq \eta t_{\mathrm{MX}}^{\perp}
$$

By solving these two equations simultaneously we obtain the primitive vectors of the supercell, $\mathbf{T}_{1}=\mathbf{t}_{\overline{\mathrm{C}}}^{\|}$and $\mathbf{T}_{2}=\mathbf{t}_{\overline{\mathrm{C}}}^{\perp}$ and the substrate lattice constant should be rescaled to $t_{\overline{\mathrm{C}}}^{\|} / t_{\mathrm{MX}}^{\|}$along one edge and $t_{\overline{\mathrm{C}}}^{\perp} / t_{\bar{M} X}^{\perp}$ in the orthogonal direction. Since the vectors $t_{\bar{C}}^{\|}$and $t_{M X}^{\|}$will in general not be aligned we need to rotate one of the layers through the angle $\theta$ between $\mathbf{t}_{\overline{\mathrm{C}}}^{\|}$and $\mathbf{t}_{\mathrm{MX}}^{\|}$

$$
\theta=\cos ^{-1} \frac{\mathbf{t}_{\overline{\mathrm{C}}}^{\|} \cdot \mathbf{t}_{\mathrm{MX}}^{\|}}{t_{\overline{\mathrm{C}}}^{\|} t_{\mathrm{MX}}^{\|}} .
$$

to construct the supercell. The resulting $\bar{C} \mid S n O$ supercell with primitive supercell vectors $\mathbf{T}_{1}=7 \mathbf{a}_{1}$ and $\mathbf{T}_{2}=2 \mathbf{a}_{1}-4 \mathbf{a}_{2}$ is shown in Fig. 5.1 . It leads to a very acceptable strain of -0.4 and 0.6 percent along orthogonal directions and contains 56 carbon atoms, 20 oxygen atoms, and $20 \mathrm{Sn}$ atoms. The layers are rotated relative to one another through $\theta=3.4^{\circ}$

To construct a trilayer unit cell with inversion symmetry (IS), we move the center of a graphene hexagon to the center of the unit cell by moving the graphene layer by $\mathbf{r}=\left(\mathbf{T}_{1}+\mathbf{T}_{2}\right) / 2+\boldsymbol{\Delta}$ where $\boldsymbol{\Delta}$ is the vector that connect the two sublattices of graphene. This construction implies that the center of inversion will be at the center of the unit cell and therefore $M$ and $X$ atoms in the inverted layer can be obtained by $\mathbf{r}_{\mathrm{X}(\mathrm{M})}^{I}=\left(\mathbf{T}_{1}+\mathbf{T}_{2}\right)-\mathbf{r}_{\mathrm{X}(\mathrm{M})}$ and the $\mathrm{z}$-component of the $\mathrm{X}$ and $\mathrm{M}$ atoms should be inverted through the graphene layer.

\subsection{Band structure and electronic properties of MX}

Litharge $\mathrm{SnO}$ and $\mathrm{PbO}$ have similar lattices and electronic band structures. Since the atomic SO splitting of oxygen is $37 \mathrm{meV}$, of $\mathrm{Sn} 442 \mathrm{meV}$, and of $\mathrm{Pb} 1.3 \mathrm{eV}$, we expect that for bands with predominantly oxygen character, the SOC induced bandsplitting will be negligible and relatively large for bands with $\mathrm{Pb}$ and $\mathrm{Sn} p$ character. The bulk band structures of $\mathrm{SnO}$ and $\mathrm{PbO}$ are shown in the Fig. 5.2. We present the 
(a)

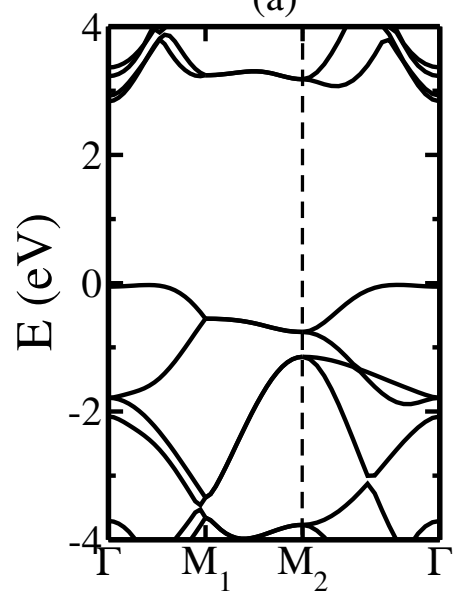

(b)

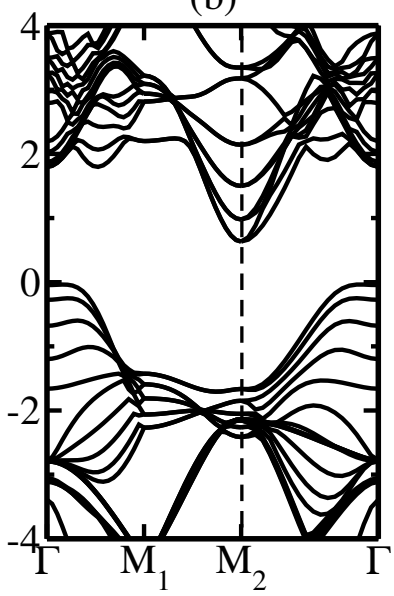

Figure 5.3: The band structures of (a) a monolayer and (b) five layers of SnO.
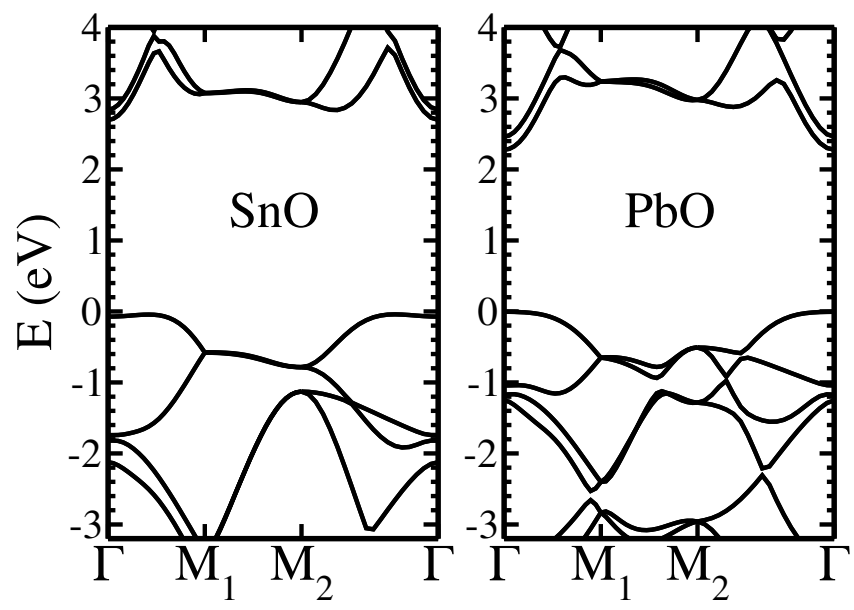

Figure 5.4: Comparison of the band structures of monolayer (a) $\mathrm{SnO}$ and (b) $\mathrm{PbO}$. $\Gamma$ is the center of Brillouin zone, $M_{1}$ a high-symmetry point and $\mathrm{M}_{2}$ is the corner of Brillouin zone.

lattice parameters and work functions og graphene and MX in Table. 5.1.

Since the bulk band gap of $\mathrm{SnO}$ is only about $0.4 \mathrm{eV}$, semi-infinite $\mathrm{SnO}$ with this small band gap will not be an appropriate substrate since the Dirac cone of graphene might overlap the $\mathrm{SnO}$ bands. The band structures of a monolayer and of five monolayers of SnO plotted in Fig. 5.3 show that a monolayer of $\mathrm{SnO}$ has a large and quite uniform (in k-space) band gap of $\sim 3 \mathrm{eV}$. Comparison of Figs. 5.4 and 5.5 show that stacking $\mathrm{SnO}$ trilayers leads to a large dispersion normal to the monolayer plane es- 


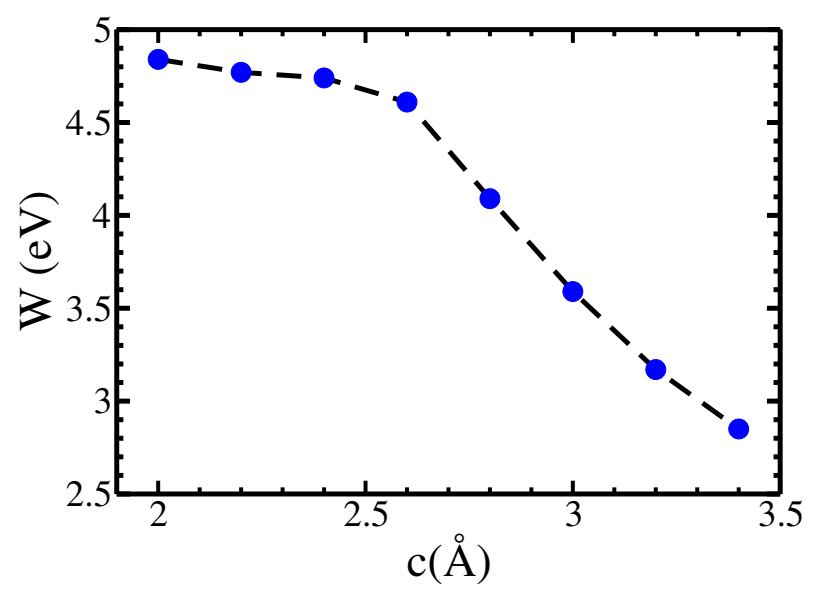

Figure 5.5: The work function of monolayer- $\mathrm{PbO}$ as a function of the separation of $\mathrm{Pb}-\mathrm{Pb}$ layers $c$ normal to the plane of the layer. The workfunction for the calculated equilibrium value of $c_{\mathrm{eq}}=2.39 \AA$ is around $4.76 \mathrm{eV}$ and reaches a maximum of 4.9 $\mathrm{eV}$.

pecially at the $M_{2}$ point (corner of Brillouin zone) where the conduction band edge is seen to descend towards the Fermi level. The $2.9 \mathrm{eV}$ large band gap of a SnO monolayer will decrease as a function of the number of layers toward $0.4 \mathrm{eV}$, the bulk value. From this observation we conclude that in an experiment graphene should be deposited on at most a few layers of $\mathrm{SnO}$ (on a large band gap substrate such as $h$-BN). Explicit calculations show that for graphene on a semi-infinite SnO substrate, the Dirac cone falls below the Fermi energy and overlaps the occupied SnO bands.

The band gap of a monolayer of $\mathrm{PbO}$ is around $2.3 \mathrm{eV}$ but its work function is much lower than that of $\mathrm{SnO}$. The low workfunction of $\mathrm{PbO}$ and formation of an interface dipole layer combine so that graphene on $\mathrm{PbO}$ is electron doped. Under external pressure the workfunction can increase as shown in Fig. 5.5, but reaches a maximum of $4.9 \mathrm{eV}$ where the Dirac cone in $\overline{\mathrm{C}} \mid \mathrm{PbO}$ is still doped. As a result we do not continue our calculations with $\overline{\mathrm{C}} \mid \mathrm{PbO}$ and focus on the $\overline{\mathrm{C}} \mid \mathrm{SnO}$ system.

\subsection{Phenomenological model}

In this section we introduce an effective four-band Hamiltonian for graphene on a $\mathrm{SnO}$ substrate and sandwiched between $\mathrm{SnO}$ layers. We use first-principles calculations to find the parameters in the Hamiltonian and use these to calculate the topological invariants of both systems. 


\subsubsection{Inversion symmetric (IS) supercell}

We start by considering IS $\mathrm{SnO}|\overline{\mathrm{C}}| \mathrm{SnO}$ trilayers since the effective Hamiltonian is made simpler by inversion symmetry. In Chapter 4 we showed that the effective Hamiltonian for a trilayer could be written

$$
H_{\mathbf{K}}^{\mathrm{IS}}(\mathbf{q})=\left(\hbar v_{F} \mathbf{q}+\mathbf{f}_{1}\right) \cdot \boldsymbol{\sigma}+\boldsymbol{\lambda}_{\mathrm{so}} \cdot \mathbf{s}_{z} \sigma_{z}
$$

where $\sigma$ and $\mathrm{s}$ are vectors of Pauli matrices representing sublattice and spin degrees of freedom, respectively. The linear term, $\hbar v_{F} \mathbf{q}$, has the form of the unperturbed Hamiltonian for a graphene monolayer. $\mathbf{f}_{1}$ is a two-dimensional vector which can result from breaking the $C_{3}$ symmetry of graphene. It in turn will break the symmetry of the (unperturbed) bands around the $\mathbf{K}$ point. If it is nonzero, the bands will exhibit a direction dependence; however, the band structure will be symmetric about the point $\mathbf{q}=-\mathbf{f}_{1} / \hbar v_{\mathrm{F}}$ and $\mathbf{f}_{1}$ will not open a gap. The term $\mathbf{f}_{1} \cdot \boldsymbol{\sigma}$ is even under inversion. $\boldsymbol{\lambda}_{\mathrm{so}}$ is a generalized three-dimensional Kane and Mele [30, 29] SOC term which contains in addition to the intrinsic spin-orbit interactions of graphene, a contribution induced by the substrate.

The eigenvalues of Eq. 5.2 are

$$
\varepsilon= \pm \sqrt{\left|\hbar v_{F} \mathbf{q}+\mathbf{f}_{1}\right|^{2}+\lambda_{\mathrm{so}}^{2}}
$$

where all states are doubly degenerate because of the time-reversal and inversion symmetries. The system will be gapless only if $\boldsymbol{\lambda}_{s o}=\mathbf{0}$, moreover the complete phase space has topological insulating character (TI). Since the bands are doubly degenerate their spin character will be lost because each pair of bands comprises states with opposite spin character so spin matrices cannot be defined uniquely. The two eigenvalues in Eq. 5.3 at the $\mathbf{K}$ point are not sufficient to uniquely determine the five parameters in Eq. 5.2. In the weak coupling (of graphene and $\mathrm{SnO}$ ) limit, we expect the contributions of each $\mathrm{SnO}$ layer to be additive. Since each term in Eq. (5.3) is even under inversion, the value of these parameters for AS bilayers will be half of the value for the corresponding IS trilayer in this limit. We calculate a gap of $\sim 0.7 \mathrm{meV}$ at the $\mathbf{K}$ point for the IS system so we predict that for the AS system $\sqrt{\mathbf{f}_{1}^{2}+\boldsymbol{\lambda}_{\mathrm{so}}^{2}} \sim 0.2 \mathrm{meV}$. This result will help us to find the correct parameters for the AS system which are consistent with the IS gap.

The topological nature of the IS trilayer can be explicitly calculated from firstprinciples wavefunctions because there is no ambiguity in the Pfaffian when a system has inversion symmetry. The topological invariant is related to the parity eigenvalues of each band [32]. For each fixed point $\boldsymbol{\Gamma}_{i}$ we can calculate

$$
\delta_{i}=\prod_{m=1}^{N} \xi_{2 m}\left(\boldsymbol{\Gamma}_{i}\right)
$$

where $\xi_{2 m}\left(\Gamma_{i}\right)$ is the parity eigenvalue of bands $2 m-1$ and $2 m$ at $\boldsymbol{\Gamma}_{i}$; since the two 
bands are degenerate and have the same parity at these points, only one eigenvalue of each pair is needed to obtain $\delta_{i} .2 N$ is the number of occupied bands. However, in a first-principles numerical calculation states are only degenerate to within some tolerance that depends on the details of the numerical algorithms etc. If there are other bands close to the $(2 m-1,2 m)$ pair, this can cause some ambiguity in determining the sign of $\delta_{i}$. This can occur if a crystal has other symmetry operators in the $\boldsymbol{\Gamma}_{i}$ symmetry group so that a higher-order degeneracy would occur in the absence of SOC. With SOC included, these degeneracies may be lifted but the eigenvalues may still be very close to each other if the spin-orbit interaction is weak. To avoid such problems we prefer to count the number of pairs $(s)$ with negative parity eigenvalues taking into account all the bands and not only the bands with even (odd) band number. We found that

$$
s_{i}=\frac{2 N-\sum_{m=1}^{2 N} \xi_{m}\left(\boldsymbol{\Gamma}_{i}\right)}{4}
$$

and therefore $(-1)^{\delta_{i}}=(-1)^{s_{i}}$, where the $\mathbb{Z}_{2}$ invariant is $(-1)^{\nu}=\prod_{i=1}^{4} \delta_{i}$. We found that for the IS-SnO $|\overline{\mathrm{C}}| \mathrm{SnO}$ trilayer, the invariant is 1 and therefore this system is a topological insulator. The good agreement between the Hamiltonian parameters determined for AS bilayer and IS trilayers and the prediction of the topological phase for the IS system from the effective Hamiltonian, allows us to claim that our phase space analysis of the effective Hamiltonian for the AS- $\overline{\mathrm{C}} \mid \mathrm{SnO}$ and prediction of the topological invariant are correct.

\subsubsection{Asymmetrical supercell (AS)}

To describe the low energy bands of graphene around the $\mathbf{K}$ point, we use the most general four band Hamiltonian because the AS- $\overline{\mathrm{C}} \mid \mathrm{SnO}$ system has no symmetry besides time-reversal. The Hamiltonian can be written as

$$
\begin{aligned}
H_{\mathbf{K}}^{\mathrm{AS}}(\mathbf{q})= & \left(\hbar v_{\mathrm{F}} \mathbf{q}+\mathbf{f}_{1}\right) \cdot \boldsymbol{\sigma}+\lambda_{m} \sigma_{z}+\boldsymbol{\lambda}_{\mathrm{so}} \cdot \mathbf{s} \sigma_{z}+\boldsymbol{\lambda}_{B} \cdot \mathbf{s}+\mathbf{f}_{2} \cdot \boldsymbol{\sigma} s_{z}+\frac{\lambda_{R}}{2}\left(\sigma_{x} s_{y}-\sigma_{y} s_{x}\right) \\
& +\frac{\bar{\lambda}_{R}}{2}\left(\sigma_{x} s_{y}+\sigma_{y} s_{x}\right)+\frac{\lambda_{r}}{2}\left(\sigma_{x} s_{x}-\sigma_{y} s_{y}\right)+\frac{\bar{\lambda}_{r}}{2}\left(\sigma_{x} s_{x}+\sigma_{y} s_{y}\right)
\end{aligned}
$$

$\lambda_{m} \sigma_{z}$ is the "mass" term that represents sublattice symmetry breaking that can be caused by the formation of a dipole layer between graphene and $\mathrm{SnO}$ or by any other asymmetric interaction of the graphene sublattices with the substrate; it is odd under inversion. $\boldsymbol{\lambda}_{B}$ is a three vector that corresponds to an effective magnetic field; it is also odd under inversion. $\mathbf{f}_{2}$, like $\mathbf{f}_{1}$, can result from breaking of $C_{3}$ rotational symmetry. It breaks the symmetry of the bands about the $\mathbf{K}$ point, displacing the Dirac point to $\mathbf{q}=-\mathbf{f}_{2} / \hbar v_{\mathrm{F}}$ for up spins and to $\mathbf{q}=\mathbf{f}_{2} / \hbar v_{\mathrm{F}}$ for down spins; it is odd under inversion.

The $\lambda_{R}, \bar{\lambda}_{R}, \lambda_{r}$, and $\bar{\lambda}_{r}$ ("Rashba") terms correspond to an effective electric field between the layers. If an electric field is applied perpendicular to a single layer of graphene then the $\lambda_{R}$ term ("Rashba") represents the effect of the field at $\mathbf{K}$. If the 
(a)

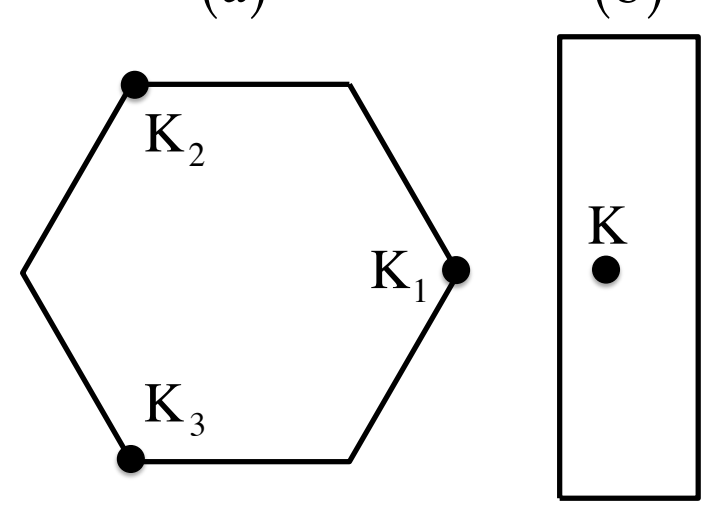

(b)

Figure 5.6: Brillouin zone of (a) monolayer-graphene and (b) AS-C $\mid \mathrm{SnO} . \mathbf{K}_{1}, \mathbf{K}_{2}$, and $\mathbf{K}_{3}$ all map into the $\mathbf{K}$ point of $\mathrm{AS}-\overline{\mathrm{C}} \mid \mathrm{SnO}$.

electric field is caused by interactions with a substrate or other orbitals then its representation will depend on the $\mathbf{K}$ point position. To illustrate this point, we consider a free-standing buckled monolayer of hexagonal boron nitride $(h$-BN) because, in order to observe the Rashba term, we must break inversion and mirror symmetry. In the absence of spin-orbit interactions and buckling, the $p_{z}$ orbitals of flat $h$-BN will be completely decoupled from the $s p^{2}$ orbitals. Buckling, whereby the B and N sublattices are displaced by equal and opposite amounts normal to the plane, will allow mixing between $p_{z}$ and $s p^{2}$ orbitals. As a result, the three equivalent $\mathbf{K}$ points in the Brillouin zone will no longer have the same representation because $p_{x}$ and $p_{y}$ orbitals mix under $C_{3}$. For a buckled sheet of $h$-BN with SOC, the Hamiltonian at the three $\mathbf{K}$ points in Fig. 5.6(a) are related to each other by

$$
\begin{array}{ll}
H_{\mathbf{K}_{1}}= & H_{i}+\frac{\lambda_{R}}{2}(\boldsymbol{\sigma} \times \mathbf{s})_{z} \\
H_{\mathbf{K}_{2}}= & H_{i}+\frac{\lambda_{R}}{2}\left[-\frac{1}{2}(\boldsymbol{\sigma} \times \mathbf{s})_{z}-\frac{\sqrt{3}}{2}(\boldsymbol{\sigma} . \mathbf{s})_{x y}\right] \\
H_{\mathbf{K}_{3}}= & H_{i}+\frac{\lambda_{R}}{2}\left[-\frac{1}{2}(\boldsymbol{\sigma} \times \mathbf{s})_{z}+\frac{\sqrt{3}}{2}(\boldsymbol{\sigma} . \mathbf{s})_{x y}\right]
\end{array}
$$

where $H_{i}=\lambda_{m} \sigma_{z}+\lambda_{s o} \sigma_{z} s_{z}+\lambda_{B} s_{z}$ and $(\boldsymbol{\sigma} . \mathbf{s})_{x y}=\sigma_{x} s_{x}+\sigma_{y} s_{y}$. Eq. 5.7 shows that for buckled $h$-BN, $\lambda_{R}$ and $\bar{\lambda}_{r}$ mix at $\mathbf{K}_{2}$ and $\mathbf{K}_{3}$. In the absence of $C_{3}$ both terms mix; the two terms cannot be reduced to a single term. If we consider the $\mathbf{K}^{\prime}$ points, we will see that $\bar{\lambda}_{R}$ and $\lambda_{r}$ also mix so the most general representation of the Rashba term will contain all $\lambda_{R}, \bar{\lambda}_{R}, \lambda_{r}$, and $\bar{\lambda}_{r}$ terms. In our AS- $\bar{C} \mid$ SnO supercell all three $\mathbf{K}$ points map into a single point and the most general form of the Rashba term must 
be used.

To fit the band structure resulting from a first-principles calculation, we need information about the bands and the wave functions at the $\mathbf{K}$ point. There are four eigenvalues at the $\mathbf{K}$ point but because the effective Hamiltonian Eq. (5.6) does not contain a $\sigma_{o} s_{o}$ term, the sum of the eigenvalues is zero yielding three equations related to the energies. More information is to be had about the system by examining the spin matrices for the four bands. The first-principles wave functions have the form $\tilde{\psi}_{n \mathbf{k}}(\mathbf{r})=\left(\tilde{\psi}_{n \mathbf{k}}^{\uparrow}(\mathbf{r}), \tilde{\psi}_{n \mathbf{k}}^{\downarrow}(\mathbf{r})\right)$, where each spin component is expanded in a plane wave basis as $\tilde{\psi}_{n \mathbf{k}}^{\sigma}(\mathbf{r})=\sum_{\mathbf{g}_{i}} C_{n \mathbf{k}}^{\sigma \mathbf{g}_{i}} e^{i\left(\mathbf{k}+\mathbf{g}_{i}\right) \cdot \mathbf{r}}$ where $\sigma$ is the spin component of the wavefunction and the summation is over reciprocal lattice vectors $\mathbf{g}_{i}$. The projection operator for the $z$ component of spin is simply $\sigma_{o} s_{z}$ and therefore we have

$$
\left\langle\tilde{\psi}_{m \mathbf{K}}\left|\sigma_{o} s_{z}\right| \tilde{\psi}_{n \mathbf{K}}\right\rangle=\sum_{\mathbf{g}_{i}}\left[\left(C_{m \mathbf{K}}^{\uparrow \mathbf{g}_{i}}\right)^{*} C_{n \mathbf{K}}^{\uparrow \mathbf{g}_{i}}-\left(C_{m \mathbf{K}}^{\downarrow \mathbf{g}_{i}}\right)^{*} C_{n \mathbf{K}}^{\downarrow \mathbf{g}_{i}}\right]
$$

and for $\sigma_{o} s_{x}$

$$
\left\langle\tilde{\psi}_{m \mathbf{K}}\left|\sigma_{o} s_{x}\right| \tilde{\psi}_{n \mathbf{K}}\right\rangle=\sum_{\mathbf{g}_{i}}\left[\left(C_{m \mathbf{K}}^{\uparrow \mathbf{g}_{i}}\right)^{*} C_{n \mathbf{K}}^{\downarrow \mathbf{g}_{i}}+\left(C_{m \mathbf{K}}^{\downarrow \mathbf{g}_{i}}\right)^{*} C_{n \mathbf{K}}^{\uparrow \mathbf{g}_{i}}\right]
$$

For the equilibrium separation of AS- $\overline{\mathrm{C}} \mid \mathrm{SnO}$ we calculated the corresponding $s_{z}$ and $s_{x}$ matrices. The Hamiltonian $H$ has been transformed by a unitary matrix $U$ as $U^{\dagger} H U=H^{D}$ where $H^{D}$ is a diagonal matrix and its diagonal elements are the eigenvalues calculated from first-principles. Therefore the spin matrices transform with the same unitary matrix, $U^{\dagger} \sigma_{o} s_{z} U=S_{z}^{U}$ where $S_{z}^{U}$ is calculated with the firstprinciples wave functions from Eq. 5.8. However, since the eigenvalues of $\sigma_{o} s_{z}$ and $S_{z}^{U}$ are degenerate, their transformation matrix is not uniquely determined. In general we have

$$
U^{\dagger}\left(\begin{array}{cc}
\omega_{1}^{\dagger} & 0_{2 \times 2} \\
0_{2 \times 2} & \omega_{2}^{\dagger}
\end{array}\right) \sigma_{o} s_{z}\left(\begin{array}{cc}
\omega_{1} & 0_{2 \times 2} \\
0_{2 \times 2} & \omega_{2}
\end{array}\right) U=S_{z}^{U}
$$

where $\omega_{1}$ and $\omega_{2}$ are two independent $2 \times 2$ unitary matrices and they will be completely undetermined by Eq. 5.10. If we choose a unitary matrix $V$ that diagonalizes $S_{z}^{U}\left(V S_{z}^{U} V^{\dagger}=s_{z}\right)$ then we have

$$
V=\left(\begin{array}{cc}
\omega_{1} & 0 \\
0 & \omega_{2}
\end{array}\right) U
$$

Applying $V$ to the spin in the $x$ direction, the $\sigma_{o} s_{x}$ transformation is

$$
V S_{x}^{U} V^{\dagger}=\left(\begin{array}{cc}
\omega_{1} & 0 \\
0 & \omega_{2}
\end{array}\right) U S_{x}^{U} U^{\dagger}\left(\begin{array}{cc}
\omega_{1}^{\dagger} & 0 \\
0 & \omega_{2}^{\dagger}
\end{array}\right)=S_{x}^{V}
$$


and since the $U S_{x}^{U} U^{\dagger}=\sigma_{o} s_{x}$ we have

$$
S_{x}^{V}=\left(\begin{array}{cc}
0 & \omega_{1} \omega_{2}^{\dagger} \\
\omega_{2} \omega_{1}^{\dagger} & 0
\end{array}\right)=\left(\begin{array}{cc}
0 & \tilde{s}_{x} \\
\tilde{s}_{x} & 0
\end{array}\right)
$$

where $\tilde{s}_{x}$ is determined from the first-principles spin matrices and the chosen $V$ matrix. One can show that $\tilde{s}_{x}$ is unitary: $\tilde{s}_{x} \tilde{s}_{x}^{\dagger}=\omega_{1} \omega_{2}^{\dagger} \omega_{2} \omega_{1}^{\dagger}=s_{o}$ and therefore $\omega_{1}$ can be related to $\omega_{2}$ by $\omega_{1}=\tilde{s}_{x} \omega_{2}$. Therefore the matrix $U$ is

$$
U=\left(\begin{array}{cc}
\omega_{2}^{\dagger} & 0 \\
0 & \omega_{2}^{\dagger}
\end{array}\right)\left(\begin{array}{cc}
\tilde{s}_{x}^{\dagger} & 0 \\
0 & 1
\end{array}\right) V=\left(\begin{array}{cc}
\omega_{2}^{\dagger} & 0 \\
0 & \omega_{2}^{\dagger}
\end{array}\right) W
$$

If we define $H^{W}=W H^{D} W^{\dagger}$ then the Hamiltonian is

$$
H_{\mathbf{K}}^{\mathrm{AS}}(\mathbf{q})=\left(\omega_{2}^{\dagger} \otimes s_{o}\right) H^{W}\left(\omega_{2} \otimes s_{o}\right)+\hbar v_{F} \mathbf{q} \cdot \boldsymbol{\sigma}
$$

$H^{W}$ has the same eigenvalues and spin matrices as $H$ has at the $\mathbf{K}$ point. We next make use of the derivatives of the energies with respect to q. Since a unitary transformation will not change the eigenvalues we are allowed to use a different representation for $H$ as long as we apply it to the linear term too. We then have

$$
H^{\omega}=\omega_{2} H_{\mathbf{K}}^{\mathrm{AS}} \omega_{2}^{\dagger}=H^{W}+\hbar v_{F} \mathbf{q} \cdot\left(\omega_{2} \boldsymbol{\sigma} \omega_{2}^{\dagger}\right)
$$

Any $2 \times 2$ unitary matrix can be expanded using Pauli matrices as

$$
\omega_{2}=a_{o} \sigma_{o}+i a_{x} \sigma_{x}+i a_{y} \sigma_{y}+i a_{z} \sigma_{z}
$$

where $a_{i}$ are real with the constraint that $\sum_{i} a_{i}^{2}=1$. Applying $\omega_{2}$ to $\sigma_{x}$ yields

$$
\begin{aligned}
\omega_{2} \sigma_{x} \omega_{2}^{\dagger} & =\left(a_{o}^{2}+a_{x}^{2}-a_{y}^{2}-a_{z}^{2}\right) \sigma_{x} \\
& +\quad 2\left(a_{x} a_{y}+a_{o} a_{z}\right) \sigma_{y}+2\left(a_{x} a_{z}-a_{o} a_{y}\right) \sigma_{z}
\end{aligned}
$$

and for the $y$ direction

$$
\begin{aligned}
\omega_{2} \sigma_{y} \omega_{2}^{\dagger}= & 2\left(a_{x} a_{y}-a_{o} a_{z}\right) \sigma_{x}+\left(a_{o}^{2}-a_{x}^{2}+a_{y}^{2}-a_{z}^{2}\right) \sigma_{y} \\
& +\quad 2\left(a_{o} a_{x}+a_{y} a_{z}\right) \sigma_{z}
\end{aligned}
$$

By using the eigenvectors of $H^{W}$ we can relate the derivatives of energies to Eq. 5.18 and Eq. 5.19 as

$$
\frac{\partial \varepsilon_{n}}{\partial \mathbf{q}}=\left\langle\varphi_{n}^{W}\left|\omega_{2} \boldsymbol{\sigma} \omega_{2}^{\dagger}\right| \varphi_{n}^{W}\right\rangle
$$

This equation yields six relations (three for each direction) for $a_{i}$. By solving them we can find the $\omega_{2}$ matrix and therefore determine the Hamiltonian Eq. 5.15 that will automatically give the exact eigenvalues, spin-matrices, and derivatives of the bands 


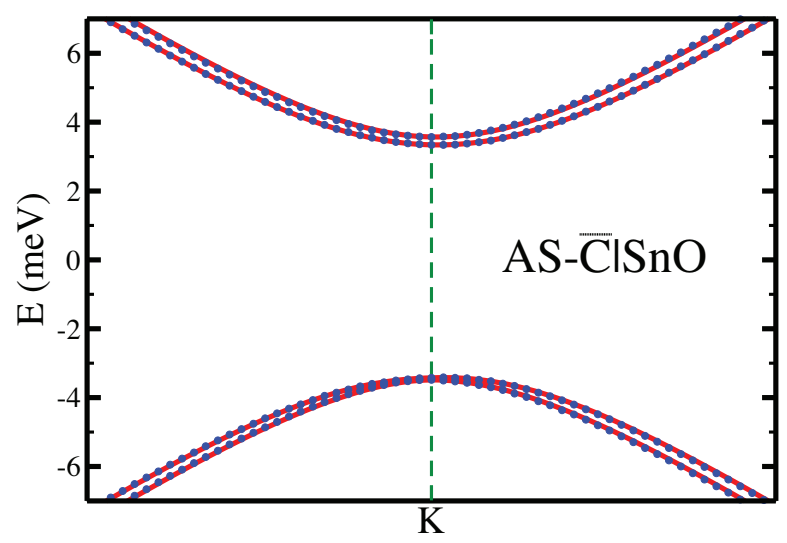

Figure 5.7: Band structure of AS- $\bar{C} \mid S n O$ close to the $\mathbf{K}$ point in the $\boldsymbol{\Gamma}-\mathbf{K}$ direction. The blue balls represent the first-principles calculation results, the red line represents the bands from the model.

around the $\mathbf{K}$ point.

The first-principles bands are compared with the bands from Eq. 5.15 close to the $\mathbf{K}$ point in Fig. 5.7 in the $\mathbf{\Gamma}-\mathbf{K}$ direction. The band structure is slightly asymmetric around the $\mathbf{K}$ point and the derivatives of the bands in the $\boldsymbol{\Gamma}-\mathbf{K}$ direction are slightly different from the bands at the perpendicular direction. This is reflected in the parameters since $\mathbf{f}_{1}$ and $\mathbf{f}_{2}$ are non-zero. The dominant parameters for AS-C $\mid$ SnO are the Rashba terms, $\lambda_{R}, \bar{\lambda}_{r}$ terms and their corresponding complementary representations (Eq. 5.7) which indicate strong breaking of the reflection symmetry. The band gap of AS system is $6.8 \mathrm{meV}$ and the parameters for the AS bilayer predict a gap of $0.7 \mathrm{meV}$ for the corresponding IS trilayer which is in good agreement with the value of $0.7 \mathrm{meV}$ that we found for the IS system by explicit calculation. The origin of the large difference between AS and IS band gaps is that in AS system the terms that contribute the most to the gap opening are odd under inversion. Since the Rashba terms are also odd under reflection symmetry then we expect that for reflection symmetric (RS) systems the value of band gap be close to the IS gaps. Therefore making any sandwich of $\mathrm{MX}|\overline{\mathrm{C}}| \mathrm{MX}$ will most likely reduce the band gap.

The first-principles wave functions cannot be directly used to obtain the $\mathbb{Z}_{2}$ invariant $(\nu)$ in the absence of inversion symmetry; we instead use the phase space of the effective Hamiltonian. For AS- $\overline{\mathrm{C}} \mid \mathrm{SnO}$ this phase space depends on fifteen independent parameters and therefore forms a fifteen dimensional space. Direct inspection of the topological invariant is therefore not practical. There are two limits where we know the topological invariant. When $\lambda_{m} \rightarrow \pm \infty$ the system will be a normal insulator (NI) with $\nu=0$; when $\lambda_{\text {so }}^{(z)} \rightarrow \pm \infty$ the system is a topological insulator (TI) with $\nu=1$. These four points are separated from each other by a boundary where the gap of the system vanishes. Any point that can be connected to one of the NI 


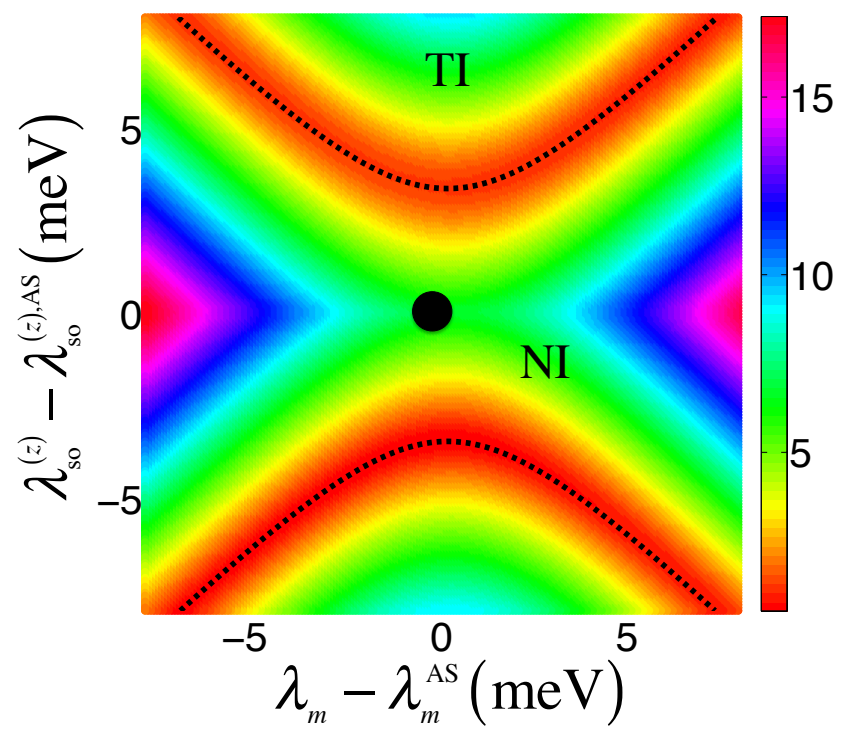

Figure 5.8: Band gap in a $\lambda_{m}-\lambda_{\text {so }}^{(z)}$ cross section of the phase space of AS $\bar{C} \mid \operatorname{SnO}$ where the origin (black disk) represents the parameters we extract from the first-principles calculations. The TI and NI regions are separated by red region where the gap vanishes. The color code gives the magnitude of the gap. The dashed-black curves are approximate solutions of zero-gap condition.

points without closing the gap of the system will itself be a NI while any point that can be connected to one of the TI points without closing the gap is a TI. In Fig. 5.8 we show the phase space (and boundaries where the gap is zero) in the $\lambda_{m}-\lambda_{\text {so }}^{(z)}$ plane around the point where our system is located. The origin represents the parameters that we calculate from first principles for the AS- $\overline{\mathrm{C}} \mid \mathrm{SnO}$ system. Two TI and NI regions are indicated in the figure, separated by red regions where the band gap vanishes. An approximate solution to the gapless curves is

$$
\lambda_{s o}= \pm \sqrt{\lambda_{m}^{2}+\lambda_{R}^{2}+\bar{\lambda}_{r}^{2}}
$$

and this solution is shown by dashed-black curves in Fig. 5.8. According to the Fig. 5.8, the system is located in a NI region and therefore the AS- $\bar{C} \mid \mathrm{SnO}$ should be a normal insulator with a $6.8 \mathrm{meV}$ band gap.

\subsection{Conclusion}

We explored the idea of inducing large spin-orbit interactions in graphene by letting it hybridize with a compound that contains heavy metal elements on the outside. Because such a system in general might not have any symmetry, a general four-band 
phenomenological low-energy Hamiltonian had been applied for an asymmetric bilayer and an inversion symmetric trilayer in order to understand the interactions in the system and test the Hamiltonian. Three new terms have been introduced in the Hamiltonian that represent a generalized Rashba interaction. For simple systems where $C_{3}$ symmetry is only weakly broken, a single new term suffices to describe the band structure and spin characters of the bands, but for a more complicated system like AS- $\overline{\mathrm{C}} \mid \mathrm{SnO}$ where all $\mathrm{K}$ points map into one point in the supercell Brillouin zone (SBZ) and $C_{3}$ is broken, then all of the Rashba representations have to be included in the Hamiltonian.

Our first-principles calculations showed that the work function of litharge $\mathrm{PbO}$ is too small to be used as a substrate for graphene. Because of the low work function of $\mathrm{PbO}$ and the formation of a dipole layer, the graphene cone will be doped. To our surprise $\mathrm{SnO}$ has a higher work function by comparison with $\mathrm{PbO}$ and a few layers of it can be used as a substrate for graphene. We found that AS- $\bar{C} \mid \mathrm{SnO}$ at its equilibrium separation will have $6.8 \mathrm{meV}$ gap which is dominated by Rashba interactions, indicating strong influence of an effective induced electric field. By analysing the phase space of the AS system we found that it is a normal insulator. IS$\mathrm{SnO}|\overline{\mathrm{C}}| \mathrm{SnO}$ has lower gap of $0.7 \mathrm{meV}$, however direct calculation of the topological invariant shows that it is a topological insulator.

By comparing the results of this work with comparable results for graphene TMD bilayers and trilayers, we conclude that the advantage of $\mathrm{MX}$ over $\mathrm{MX}_{2}$ is that having the metal on the outside will induce higher SOC on graphene leading to large gaps, however the Rashba terms are more likely to dominate over other parameters and change graphene into a normal insulator. 


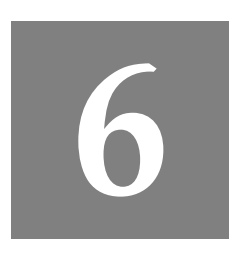

\section{$\mathbb{Z}_{2}$ invariance of germanene on $\mathrm{MoS}_{2}$ from first-principles}

We present a low energy Hamiltonian generalized to describe how the energy bands of germanene $(\overline{\mathrm{Ge}})$ are modified by interaction with a substrate. The parameters that enter the Hamiltonian are determined from first-principles relativistic calculations for $\overline{\mathrm{Ge}} \mid \mathrm{MoS}_{2}$ bilayers and $\mathrm{MoS}_{2}|\overline{\mathrm{Ge}}| \mathrm{MoS}_{2}$ trilayers. The SOC induced gap is predicted to be almost quenched by interaction with the substrate for the recently grown bilayer system. The trilayer band gap is almost as large as for a free-standing germanene layer. The full phase diagram predicts both systems to be topological insulators. 


\subsection{Introduction}

Insulators can be categorized by topological invariants that are not continuous; when these have to change, interesting physics occurs. The first group of these invariants was found to describe the quantum Hall effect for electrons confined in strong magnetic fields [36, 120, 26]. A new class of "topological" insulators (TI) was proposed for systems with time-reversal symmetry where the invariant can have two values $[29,30]$; topologically non-trivial systems are called $\mathbb{Z}_{2}$ TIs [29, 30, 25, 32, 84, 85]. In the two dimensional (2D) graphene $(\overline{\mathrm{C}})$ originally studied by Kane and Mele [29, 30], spin-orbit coupling (SOC) leads to the opening of a gap at the Dirac point giving rise to the possibility of topologically protected spin-polarized edge states. The intrinsic spin-orbit coupling (SOC) of carbon is, however, very small resulting in gaps of less than $50 \mu \mathrm{eV}(0.6 \mathrm{~K})[86,87,88,89,90]$. Two approaches have been taken to resolve this issue. One is to induce a larger spin-orbit coupling in graphene by placing it in contact with layered materials that contain heavy elements with large intrinsic SOC $[94,95,99,96,16,97,98]$. The other is to begin with a 2D group IV material with a larger intrinsic SOC $[121,14]$. Motivated by recent success in growing germanene $(\overline{\mathrm{Ge}})$ on $\mathrm{MoS}_{2}$ [122], this paper is concerned with the latter.

The structures and stability of free-standing group IV layers have already been studied theoretically. Both silicene $(\overline{\mathrm{Si}})$ and germanene "buckle" with the two sublattices moving in opposite directions out of the original plane but maintaining inversion symmetry [123, 124, 125]; stanene ( $\overline{\mathrm{Sn}})$ forms a different dumbell structure [17]. The unsupported layers are predicted to be TIs [126, 17]. Experimental efforts have so far focussed on growing silicene [127] and germanene [14] on metallic substrates where the intrinsic transport properties cannot be studied. Eventually these layered structures must be transferred to or grown on a nonconducting substrate. It is then essential to know if the TI character survives the interaction with the substrate. However, the complexity of these systems has made calculation of the topological invariant impossible until now.

We focus on the recently grown $\overline{\mathrm{Ge}} \mid \mathrm{MoS}_{2}$ system [122]. A free-standing, planar germanene layer has a SOC induced gap of $4 \mathrm{meV}$. Buckling breaks the reflection symmetry, mixes the $p_{z}$ with the $\left\{s, p_{x}, p_{y}\right\}$ orbitals and increases the SOC gap to $24 \mathrm{meV}$ [126]. It leads to one Ge sublattice interacting more strongly with a substrate than the other, breaking the sublattice symmetry and opening a gap of $20 \mathrm{meV}$ without SOC. When SOC is included, Rashba SOC is induced by the breaking of reflection (and inversion) symmetry. To investigate whether or not the gapped asymmetric bilayer is a TI, we generalize Kane and Mele's model to describe the interaction with a substrate. We use first-principles calculations to determine equilibrium geometries, evaluate the parameters in the model Hamiltonian from the first-principles electronic structures and calculate phase diagrams. We will see that the band gap induced by SOC in free-standing $\overline{\text { Ge }}$ is almost completely closed by interaction with the sublattice. By constructing an $\mathrm{MoS}_{2}|\overline{\mathrm{Ge}}| \mathrm{MoS}_{2}$ trilayer, it can be almost completely restored. The sandwich structure should also serve to stabilize and protect the $\overline{\text { Ge }}$ layer from 
the environment.

\subsection{Phenomenological model: asymmetric bilayer}

We begin by constructing a low energy Hamiltonian for graphene interacting (weakly) with a semiconducting substrate $(S)$ by downfolding a tight-binding Hamiltonian for the same system. Taking $\sigma$ and $\mathbf{s}$ to be vectors of Pauli matrices where $\sigma$ represents the $A(B)$ sublattices of graphene and s represents spin, then the result for an asymmetric (AS) $\overline{\mathrm{C}} \mid \mathrm{S}$ bilayer is

$$
H_{\mathbf{K}}^{\mathrm{AS}}(\mathbf{q})=\hbar v_{F} \mathbf{q} . \boldsymbol{\sigma}+\lambda_{m} \sigma_{z}+\frac{1}{2} \lambda_{R}(\boldsymbol{\sigma} \times \mathbf{s})_{z}+\lambda_{\mathrm{so}} \sigma_{z} s_{z}+\lambda_{B} s_{z}
$$

where $\mathbf{q}$ is the wave vector relative to the $\mathbf{K}$ point, $\mathbf{q}=\mathbf{k}-\mathbf{K} . \lambda_{m}$ is a "mass" term that describes the breaking of the sublattice symmetry by the interaction with the substrate. $\lambda_{R}$ is a Rashba SOC term that results from the breaking of reflection symmetry in the direction perpendicular to the germanene layer. $\lambda_{\text {so }}$ is Kane and Mele's spin-orbit term [29] that contains the intrinsic "atomic" SOC term of monolayer germanene plus $\lambda_{\text {so }}^{(\text {ind) }}$, the SOC induced by the substrate. $\lambda_{B}$ corresponds to a "pseudomagnetic" term which is odd under inversion symmetry and changes sign at the $\mathbf{K}^{\prime}$ point and therefore does not break time-reversal symmetry.

The eigenvalues of Eq. 6.1 at the $\mathbf{K}$ point are

$$
\begin{array}{ll}
\varepsilon_{4}= & \lambda_{\text {so }}+\lambda_{B}+\lambda_{m} \\
\varepsilon_{3}= & \lambda_{\text {so }}-\lambda_{B}-\lambda_{m} \\
\varepsilon_{2}= & -\lambda_{\text {so }}+\sqrt{\left(\lambda_{B}-\lambda_{m}\right)^{2}+\lambda_{R}^{2}} \\
\varepsilon_{1}= & -\lambda_{\text {so }}-\sqrt{\left(\lambda_{B}-\lambda_{m}\right)^{2}+\lambda_{R}^{2}}
\end{array}
$$

where the order corresponds to the results obtained in first principles calculations. By comparing these eigenvalues and the corresponding eigenvectors with values calculated from first-principles, we can determine the parameters in Eq. 6.1 with which the band structure about the Dirac point can be described. The projection of wavefunctions onto specific atoms is not unique. However, the spin space is complete to very good accuracy and we use the expectation values for the $z$ component of spin

$$
\left\langle s_{z}\right\rangle_{n \mathbf{K}}=\frac{1}{\Omega} \int_{\Omega}\left(\left|\psi_{n \mathbf{K}}^{\uparrow}(\mathbf{r})\right|^{2}-\left|\psi_{n \mathbf{K}}^{\downarrow}(\mathbf{r})\right|^{2}\right) d^{2} \mathbf{r}
$$

for the four bands at the Dirac point where the integral should be taken over the supercell with area $\Omega$. If the wavefunctions at $\mathbf{K}$ are expanded in a plane wave basis 

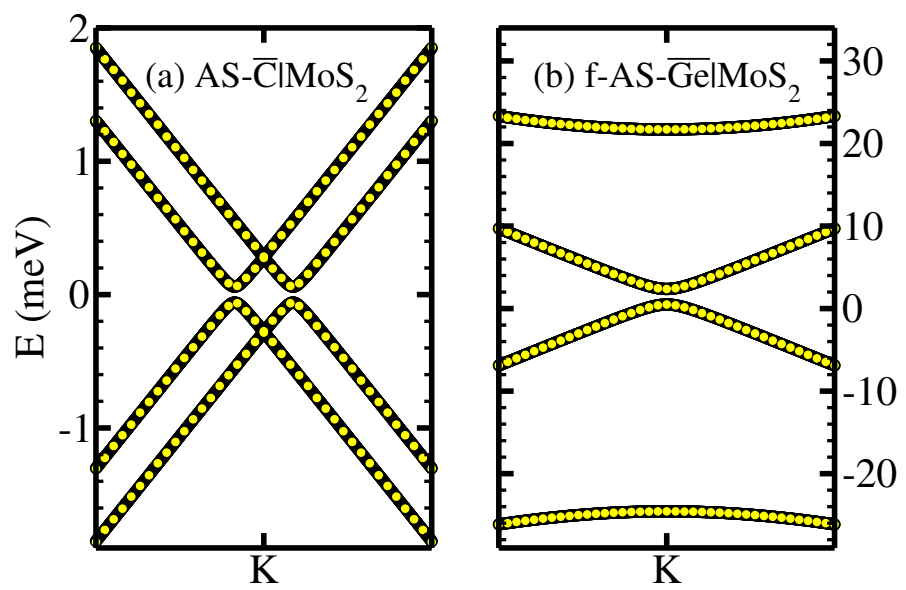

Figure 6.1: Band structures of (a) AS p- $\overline{\mathrm{C}} \mid \mathrm{MoS}_{2}$ and (b) AS b- $\overline{\mathrm{Ge}} \mid \mathrm{MoS}_{2}$ bilayers close to the $\mathbf{K}$ point. The yellow dots are the results of first principles calculations, the black lines result from the phenomenological model Eq. 6.1 with parameters from Eq. 6.5.

as $\psi_{n \mathbf{K}}(\mathbf{r})=\sum_{\sigma \mathbf{G}} C_{n \mathbf{K}}^{\sigma \mathbf{G}} e^{i(\mathbf{K}+\mathbf{G}) \cdot \mathbf{r}}$ then Eq. 6.3 simplifies to

$$
\left\langle s_{z}\right\rangle_{n \mathbf{K}}=\sum_{\mathbf{G}}\left(\left|C_{n \mathbf{K}}^{\uparrow \mathbf{G}}\right|^{2}-\left|C_{n \mathbf{K}}^{\downarrow \mathbf{G}}\right|^{2}\right)
$$

Applying Eq. 6.4 to first principles results to be presented below shows that $\left\langle s_{z}\right\rangle_{\mathbf{K}}=(s,-$ $s,-1,1)$ for the four bands at the Dirac point; here $s$ is a positive number smaller than one. Solving for the parameters in Eq. 6.1 results in

$$
\begin{array}{ll}
\lambda_{m}= & {\left[\left(\varepsilon_{4}-\varepsilon_{3}\right)+s\left(\varepsilon_{2}-\varepsilon_{1}\right)\right] / 4} \\
\lambda_{R}= & \pm\left(\varepsilon_{2}-\varepsilon_{1}\right) \sqrt{1-s^{2}} / 2 \\
\lambda_{\text {so }}= & {\left[\left(\varepsilon_{4}+\varepsilon_{3}\right)-\left(\varepsilon_{2}+\varepsilon_{1}\right)\right] / 4} \\
\lambda_{B}= & {\left[\left(\varepsilon_{4}-\varepsilon_{3}\right)-s\left(\varepsilon_{2}-\varepsilon_{1}\right)\right] / 4}
\end{array}
$$

When buckling is included, the tight-binding Hamiltonian cannot be exactly downfolded. However, it does not introduce any qualitatively new symmetries and Eq. 6.1 describes the band dispersion about the Dirac point equally well for planar $\overline{\mathrm{C}} \mid \mathrm{MoS}_{2}$ and buckled $\overline{\mathrm{Ge}} \mid \mathrm{MoS}_{2}$ as seen in Fig. 6.1. 


\subsection{Phenomenological model: inversion symmetric supercell}

he term containing $\lambda_{R}$ in Eq. 6.1 is odd under inversion and reflection. For an $\mathrm{MoS}_{2}|\overline{\mathrm{Ge}}| \mathrm{MoS}_{2}$ trilayer constructed to have inversion symmetry the average of $\lambda_{R}$ over a supercell is zero so this term is absent. The mass term $\lambda_{m}$ and pseudomagnetic term $\lambda_{\mathrm{B}}$ also vanish because they are odd under inversion and Eq. 6.1 simplifies to

$$
H_{\mathbf{K}}^{\mathrm{IS}}(\mathbf{q})=\hbar v_{F} \mathbf{q} \cdot \boldsymbol{\sigma}+\lambda_{\mathrm{so}} \sigma_{z} s_{z} .
$$

This equation satisfies the requirement of Kramers degeneracy that all bands should be doubly degenerate and predicts that the gap will vanish only if $\lambda_{\text {so }}$ is zero. In this case $\left\langle s_{z}\right\rangle$ is not uniquely defined because degenerate bands have complementary spin textures.

\subsection{First-principles calculations}

We use density functional theory (DFT) to calculate ground state energies and optimized geometries with a projector augmented wave (PAW) basis set $[8,9]$ as implemented in VASP $[58,59]$ for a $\overline{\mathrm{Ge}} \mid \mathrm{MoS}_{2}$ bilayer and an $\mathrm{MoS}_{2}|\overline{\mathrm{Ge}}| \mathrm{MoS}_{2}$ trilayer. These are repeated periodically and separated from their images by a $15 \AA$ thick vacuum region. A dipole correction is applied to avoid spurious interactions between the periodic images [81]. The plane wave kinetic energy cutoff is set at $600 \mathrm{eV}$. We apply a dense $42 \times 42 \mathbf{k}$-point grid to sample the germanene $1 \times 1$ Brillouin zone (BZ), and a comparable density for supercells. For BZ integrations we use the tetrahedron scheme [80]. The electronic self-consistency criterion is set to $10^{-7} \mathrm{eV}$. Bilayers and trilayers are relaxed until the total energy is converged to within $10^{-7} \mathrm{eV}$. As a compromise between the LDA density functional (DF) that tends to overbind, and GGA that underbinds van der Waals (vdW) structures, we used the optB88-vdW-DF $[78,102,103]$.

We first determine equilibrium geometries for individual monolayers of $\overline{\mathrm{Ge}}$ and $\mathrm{MoS}_{2}$. For germanene both planar $(\mathrm{p}-\overline{\mathrm{Ge}})$ and buckled $(\mathrm{b}-\overline{\mathrm{Ge}})$ structures are studied. For relaxed $\mathrm{b}-\overline{\mathrm{Ge}}$ the sublattice planes are separated by $c=0.71 \AA$. The calculated inplane lattice constants are 4.05, 4.05 and $3.16 \AA$ for $\mathrm{p}-\overline{\mathrm{Ge}}, \mathrm{b}-\overline{\mathrm{Ge}}$ and $\mathrm{MoS}_{2}$, respectively. We identify lattice vectors in both materials with an acceptable length mismatch and then rotate the two lattices to make them coincide. This defines a "supercell". Because of the weak interaction between germanene and $\mathrm{MoS}_{2}$ a strong preference for a particular alignment of the two lattices is not expected and this is borne out by the weak binding energy we find for the relaxed structures. We accommodate the small residual lattice mismatch in the $\mathrm{MoS}_{2}$ layer and reoptimize its structure. The $\overline{\mathrm{Ge}}$ and $\mathrm{MoS}_{2}$ layers are allowed to bond in two stages, first only changing the height of the $\mathrm{b}-\overline{\mathrm{Ge}}$ above $\mathrm{MoS}_{2}$ (h-AS structure) and then without constraint (f-AS structure). For a supercell, the average buckling is calculated as $c=\sqrt{\sum_{N_{G e}} c_{i}^{2} / N_{G e}}$ and is given together with other relevant parameters in Table 6.1. The separation of the bottom 
Table 6.1: $E_{b}$ is the binding energy in meV per $\overline{\mathrm{Ge}}$ unit cell. The dimensionless spin parameter $s$ is defined in the text. $\Delta_{\mathbf{K}}$ is the gap calculated at the $\mathbf{K}$ point in meV. The Hamiltonian parameters defined in equations (6.2) and (6.5) are given in meV for free-standing planar and buckled Ge layers, for AS $\overline{\mathrm{Ge}} \mid \mathrm{MoS}_{2}$ bilayers and for IS, RS (see text), and a completely non-symmetric (NS) $\mathrm{MoS}_{2}|\overline{\mathrm{Ge}}| \mathrm{MoS}_{2}$ trilayers. $c$ is the separation between the two Ge planes in $\AA$.

\begin{tabular}{lrrrrrrrr} 
& $E_{b}$ & $s$ & $\Delta_{\mathbf{K}}$ & $\lambda_{m}$ & $\lambda_{\text {so }}$ & $\lambda_{R}$ & $\lambda_{B}$ & $c(\AA)$ \\
\hline p-Ge & - & - & 4.21 & - & 2.11 & - & - & 0.00 \\
b-Ge & - & - & 25.78 & - & 12.89 & - & - & 0.71 \\
h-AS & 328 & 0.83 & 5.55 & 7.95 & 11.60 & 5.72 & -0.56 & 0.71 \\
f-AS & 332 & 0.87 & 1.88 & 10.28 & 12.04 & 6.18 & -0.62 & 0.73 \\
h-IS & 671 & - & 21.21 & - & 10.61 & - & - & 0.71 \\
f-IS & 680 & - & 22.71 & - & 11.36 & - & - & 0.75 \\
h-RS & 673 & 1.00 & 20.58 & 0.08 & 10.37 & 0.00 & -0.03 & 0.71 \\
h-NS & 671 & 0.99 & 21.00 & 0.26 & 10.76 & 0.03 & -0.07 & 0.71
\end{tabular}

germanene plane from the upper sulphur layer is $3.11 \AA$ for the h-AS bilayer.

For convenience, we displace the two layers in the $x y$ plane so that the threefold axes at the centre of the hexagonal rings which are also inversion centres for the component layers coincide so the final structure has threefold rotation symmetry. This allows us to generate inversion symmetric (IS) trilayers with threefold rotation symmetry that we relax in a similar fashion; first with respect to the height (h-IS) and then fully unconstrained ( $\mathrm{f}$-IS) in both cases maintaining inversion symmetry. A top view of the AS Ge $\mid \mathrm{MoS}_{2}$ bilayer supercell is shown in Fig. 6.2. The supercell contains 89 atoms and the lattice mismatch is only $0.7 \%$.

The SO splitting of the $p$ valence states of free C, Si and Ge atoms, 8.7, 32.5 and $190.2 \mathrm{meV}$, respectively has a negligible effect on the equilbrium structures of the corresponding monolayers. The increase in the atomic SOC is reflected in the SO splitting for the planar monolayers that is greatly enhanced by buckling; the $\mathbf{K}$-point SO splitting of $\mathrm{p}-\overline{\mathrm{Ge}}$ is $4.2 \mathrm{meV}$, that of $\mathrm{b}-\overline{\mathrm{Ge}}$ is $25.8 \mathrm{meV}$. For a free-standing $\overline{\mathrm{Ge}}$ layer, buckling preserves inversion symmetry so the bands at the Dirac point are linear in $q$ in the absence of SOC. The parameters extracted from the first principles calculations and Eqs. (6.2), (6.4), and (6.5) are given in Table 6.1.

\subsection{Results: AS bilayers}

The comparison in Fig. 6.1 of the first-principles band structures (yellow dots) close to the Dirac point with the results of the phenomenological model (black lines) for AS $\overline{\mathrm{C}} \mid \mathrm{MoS}_{2}$ and $\overline{\mathrm{Ge}} \mid \mathrm{MoS}_{2}$ bilayers shows that the shape of the bands changes very considerably because $\lambda_{B}$ is dominant for graphene while for germanene $\lambda_{\mathrm{so}}, \lambda_{m}$, and $\lambda_{R}$ are much larger. It is clear from the figure that the model describes the low energy bands close to the $\mathbf{K}$ point very accurately for different regimes. The firstprinciples calculations show that for $\mathrm{AS} \overline{\mathrm{Ge}} \mid \mathrm{MoS}_{2}$ the gap decreases from $5.6 \mathrm{meV}$ for 


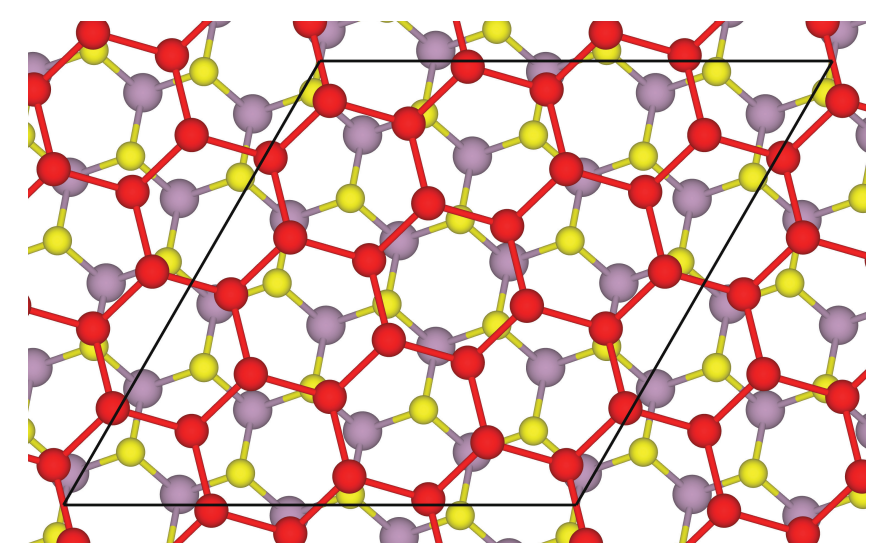

Figure 6.2: Top view of a $\overline{\mathrm{Ge}} \mid \mathrm{MoS}_{2}$ bilayer with three-fold rotation symmetry $C_{3}$ about the center of the unit cell that contains 26 Ge (red), 21 Mo (gray) and $42 \mathrm{~S}$ (yellow) atoms. The relative rotation angle is $24.8^{\circ}$.

the height optimized structure (h-AS) to $1.9 \mathrm{meV}$ for the fully unconstrained structure (f-AS); see Table 6.1. There $\lambda_{m}$ is seen to increase faster than $\lambda_{\text {so }}$ because the average buckling increases slightly from 0.71 to $0.73 \AA$ so the gap decreases. Another important point is that $\lambda_{\mathrm{so}}^{(\text {ind) }}$ is negative. Calculating $\lambda_{\mathrm{so}}^{(\mathrm{ind})}=\lambda_{\mathrm{so}}^{\mathrm{h}-\mathrm{AS}}-\lambda_{\mathrm{so}}^{\mathrm{b}-\overline{\mathrm{Ge}}}$ with parameters from Table 6.1 yields $\lambda_{\text {so }}^{\text {(ind) }}=11.60-12.89=-1.29 \mathrm{meV}$ and therefore interaction with the $\mathrm{MoS}_{2}$ layer reduces the intrinsic SOC induced gap of germanene. The mass and Rashba terms are larger than the induced SO term and both $\lambda_{m}$ and $\lambda_{R}$ increase faster than $\lambda_{\mathrm{so}}^{(\text {ind) }}$ if the interaction between germanene and $\mathrm{MoS}_{2}$ increases. Applying pressure to AS $\overline{\mathrm{G} e} \mid \mathrm{MoS}_{2}$ reduces the gap until $\lambda_{\text {so }}=$ $\frac{1}{2}\left(\lambda_{m}+\lambda_{B}+\sqrt{\left(\lambda_{m}-\lambda_{B}\right)^{2}+\lambda_{R}^{2}}\right)$ where the gap vanishes. After that, the band gap grows again but the topological nature of the bands changes. Applying pressure to AS $\overline{\mathrm{Ge}} \mid \mathrm{MoS}_{2}$ will therefore not result in a TI with a larger band gap.

To determine the $\mathbb{Z}_{2}$ topological invariant for the AS system, we analyse the phase space corresponding to Eq. 6.1 with the parameter values from Table 6.1. The TI, normal insulating (NI) and metallic (M) regions are separated by gapless curves. The $\mathbb{Z}_{2}$ invariant $(\nu)$ is related to the integral of the Berry curvature $(B)$ over the effective Brillouin zone (EBZ) and the Berry potential over its boundary [117]. In our four band model the full Brillouin zone is $\mathbf{K} \oplus \mathbf{K}^{\prime}$, the EBZ contains only $\mathbf{K}$, therefore

$$
\nu=\frac{1}{2 \pi} \int\left[B_{1}(\mathbf{q})+B_{2}(\mathbf{q})\right] d \mathbf{q}+1 \bmod 2
$$

where $B_{i}(\mathbf{q})$ is the Berry curvature of the $i^{\text {th }}$ occupied band and +1 is the contribution of the boundary. Since $\nu$ is a topological invariant it will not change unless the band gap vanishes so TI and NI regions should be separated by zero-gap lines. According to Eq. 6.7, the system will be a TI if the $\lambda_{\text {so }}$ term is dominant whereas if 


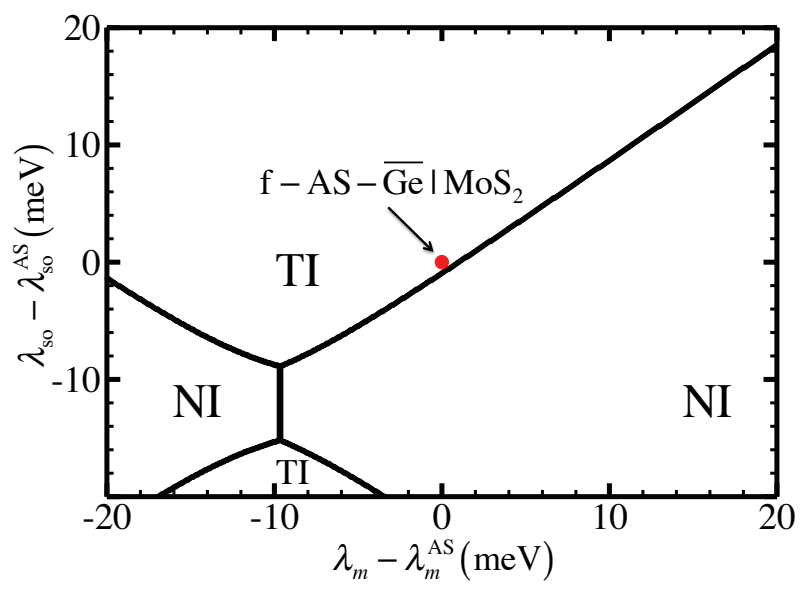

Figure 6.3: Phase space cross section for an $\mathrm{AS} \overline{\mathrm{Ge}} \mid \mathrm{MoS}_{2}$ bilayer where the origin (red dot) represents the parameter values $\left(\lambda_{m}^{\mathrm{f}-\mathrm{AS}}, \lambda_{\text {so }}^{\mathrm{f}-\mathrm{AS}}\right)=(10.28,12.04)$ calculated from first principles. Black lines represent the boundaries between regions where the gap vanishes.

$\lambda_{m}$ is dominant, the system will be a NI. Any point in the phase space that can be connected to any of the $\lambda_{\text {so }}$ dominated points without closing the gap is TI. Based on these arguments we show in Fig. $6.3 \mathrm{a} \lambda_{m}, \lambda_{\text {so }}$ cross section in the neighbourhood of the parameter values $\left(\lambda_{m}^{\mathrm{f}-\mathrm{AS}}, \lambda_{\mathrm{so}}^{\mathrm{f}-\mathrm{AS}}\right)$ we calculated for this system. According to the figure, AS Ge $\mid \mathrm{MoS}_{2}$ is a topological insulator - just. Relaxing the germanene layer fully on $\mathrm{MoS}_{2}$ does not change the $\mathbb{Z}_{2}$ invariant though the reduced gap means that it is less stable. The small size of the gap will make measurements very difficult.

\subsection{Results: IS trilayers}

Using the effective Hamiltonian parameters calculated for the AS $\overline{\mathrm{Ge}} \mid \mathrm{MoS}_{2}$ bilayer, we can estimate the band gaps at the $\mathbf{K}$ point for the IS $\mathrm{MoS}_{2}|\overline{\mathrm{Ge}}| \mathrm{MoS}_{2}$ trilayer. For the h-AS system $\lambda_{\text {so }}^{(\text {ind) }}$ was found to be $-1.29 \mathrm{meV}$. For the h-IS configuration, we predict $\lambda_{\text {so }}^{(\text {IS })}=\lambda_{\text {so }}^{(\mathrm{Ge})}+2 \lambda_{\text {so }}^{(\text {ind })}=12.89-2 \times 1.29=10.31$. An explicit first-principles calculation yields a value of $\lambda_{\mathrm{so}}^{(\mathrm{IS})}=10.61 \mathrm{meV}$. The close agreement between the predicted and calculated values indicates that the model is consistent.

In a real experiment it will not be possible to make an ideal IS trilayer; a second layer of $\mathrm{MoS}_{2}$ will most likely not be perfectly inverted with respect to the first. To check how sensitive the system is to such details, we constructed a "reflection symmetric" (RS) trilayer from the AS bilayer by reflecting the $\mathrm{MoS}_{2}$ layer in the b-Ge layer; the $b-\overline{G e}$ monolayer itself does not have reflection symmetry. For this system, the Rashba term is in practice zero and the mass term greatly reduced since there is now no interface dipole formation; see the parameter values in Table 6.1. We also 
constructed a trilayer without any symmetry (NS) to show the effect of breaking $C_{3}$ symmetry. $\lambda_{\mathrm{so}}$ does not change significantly and remains close to its IS value. We therefore predict that the band gap will be largely unchanged. This is confirmed by explicit calculation where values of $\Delta_{\mathbf{K}}=21.21,22.71,20.58$, and $21.00 \mathrm{meV}$ are found for h-IS, f-IS, h-RS and h-NS trilayers, respectively. The observation that a rotation of the second $\mathrm{MoS}_{2}$ layer will not change the electronic structure strongly indicates that the IS results can be generalized to any arbitrarily prepared encapsulated germanene structure. Our phenomenological model and its phase space predict that IS systems will be topological insulators with a substantial gap.

For inversion symmetric systems we can use the formula given by $\mathrm{Fu}$ and Kane [32] to determine the topological invariant $\nu$ explicitly from first principles calculations,

$$
(-1)^{\nu}=\prod_{i=1}^{4} \prod_{m=1}^{N} \xi_{2 m}\left(\Gamma_{i}\right)
$$

where the first multiplication is over all the time-reversal fixed points $\Gamma_{i}$ and the second multiplication is over bands with even band number at the $\Gamma_{i} ; \xi_{2 m}$ is the parity eigenvalue of bands $2 m-1$ and $2 m$. For our inversion symmetric systems, we explicitly calculated the $\mathbb{Z}_{2}$ invariant and found them all to be topological insulators with band gaps of about $23 \mathrm{meV}$ generated by SO interactions. According to this, a quantum spin Hall effect should be observable in a germanene sandwich structure at room temperature.

\subsection{Conclusion}

We use a comprehensive phenomenological model to describe spin-orbit interactions for asymmetric $\overline{\mathrm{Ge}} \mid \mathrm{MoS}_{2}$ bilayers and inversion symmetric $\mathrm{MoS}_{2}|\overline{\mathrm{Ge}}| \mathrm{MoS}_{2}$ trilayers. We determine the parameters entering this model from the eigenvalues and spin expectation values at the $\mathbf{K}$ point. The model describes the low energy band structure of germanene very accurately and provides insight into the different interactions involved. For a $\overline{\mathrm{Ge}} \mid \mathrm{MoS}_{2}$ bilayer the intrinsic SO gap of germanene is almost completely quenched by the sublattice symmetry breaking and Rashba interactions. By sandwiching $\overline{\text { Ge }}$ between $\mathrm{MoS}_{2}$ layers, we can almost completely recover the large $(23 \mathrm{meV}$ ) intrinsic SOC gap reported for free-standing germanene. We show that both the bilayer and trilayer systems are topological insulators. 


\section{Summary}

In this thesis I focussed on the interactions between graphene-like materials (graphene and germanene) and various substrates. The attractive properties of graphene like a high carrier mobility, its single-atomic thickness and its theoretical magic have made graphene a very popular and promising candidate material for numerous applications. However the zero-band gap of intrinsic graphene is a drawback for applications - if not for theoretical physicists! The study of gap opening mechanisms in graphene and how the band structure can be engineered to behave as we wish were the central questions that I have tried to address.

In chapter 2, a tight binding model was developed to describe the electronic band structure of graphene on hexagonal boron nitride $(h-\mathrm{BN})$ substrate. Since the lattice constants of graphene and $h$-BN are not exactly matched and the layers are not soft, the result of putting the two layers in contact with each other is a "superstructure" that appears in scanning tunneling microscopy studies as a moiré pattern. The number of atoms in this structure is so large that direct first-principles calculations are not possible. The alternative presented in this chapter is to use instead a minimal tight binding (TB) model. To construct the TB Hamiltonian, we need to know matrix elements of the Hamiltonian throughout the supercell and we do this by approximating the local structure with commensurable structures for which we can perform first-principles calculations. Many-body corrections to the band gap were also calculated from first-principles within the so-called GW approximation and a two-band model for these corrections was developed. The results lead us to believe that the biggest contribution to the corrections comes from electron-electron interactions in the graphene itself. After adopting the (modified) parameters into the tight binding model we found that the gap of graphene on $h$-BN is about $30 \mathrm{meV}$, in very good agreement with experiment. An effective low-energy Hamiltonian was solved for the same system to which perturbation theory was applied. The results indicated that the gap appears due to a combination of the sublattice-symmetry-breaking potential, interface dipole formation and a small but important height modulation of graphene with respect to $h$-BN. The effect of screening was studied using the Thomas-Fermi model for doped and undoped graphene. The screening length and dielectric constant were calculated for a simple and symmetric external potential.

In chapter 3, I look more closely into the formation of the dipole layer between 
graphene and $h$-BN, its magnitude and size. I also calculated the charge density distribution over the supercell for commensurable rotated layers. The results show that for large enough relative rotations the band gap vanishes. However, the potential landscape on graphene is complicated and shows that although the band structure of the system is simple, the wavefunctions are not. The analysis in this chapter shows that for graphene on $h$-BN the wavefunction follows the potentials and therefore the charge density can be an indicator of the potential landscape.

Chapter 4 is a comprehensive study of the spin-orbit interactions induced in graphene by proximity to transition metal dichalcogenide (TMD) substrates. Spinorbit coupling and time-reversal symmetry can lead to a new phase of matter, a $Z_{2}$ topological insulating phase, in such systems. This phase is interesting for theoretical research because it involves a non-trivial structure over the Hilbert space. This non-triviality will lead to the existence of robust edge states on the boundary between the material and vacuum. My study in this chapter led to the development of an effective four-band model for the low-energy band structure of graphene on TMDs which was used to predict the non-triviality of the wavefunctions. To this end, first-principle calculations were performed and from the eigenvalues and spin expectation values of the wavefunctions at the high-symmetry $\mathbf{K}$ point the parameters were derived analytically. The parameterization of the effective Hamiltonian allowed me to predict band gaps for different configurations.

In chapter 5, I continued my study of the nature of the spin-orbit interaction induced in graphene but choosing a more complicated substrate with larger intrinsic spin-orbit coupling and different rotational symmetry. The substrate I studied was $\mathrm{SnO}$ that has a square lattice which breaks the rotational symmetry of the graphene layer. I developed a method to obtain a very general four-band Hamiltonian for this system, moreover an insight into the various new terms and parameters was suggested and the relation between the terms has been explained by using simple systems.

In the last chapter, motivated by recent experimental developments in the group of Prof. Harold Zandvliet, I investigated the interaction between buckled germanene and a $\mathrm{MoS}_{2}$ substrate. I studied the effect of inversion and reflection symmetry on this structure and concluded that for germanene on $\mathrm{MoS}_{2}$ the interaction almost completely quenches the quite large intrinsic spin-orbit coupling induced band gap of free-standing germanene. By introducing a second $\mathrm{MoS}_{2}$ layer on the other side of germanene, we recover almost all of this intrinsic gap. The first-principles calculations for various $\mathrm{MoS}_{2} \mid$ Germanene $\mid \mathrm{MoS}_{2}$ sandwiches show that the band gap and parameters do not depend strongly on the precise positioning of layers and therefore for a general germanene- $\mathrm{MoS}_{2}$ sandwich we predict that the system is a topological insulator. Moreover applying external pressure can lead to a phase transition in the single layer germanene on $\mathrm{MoS}_{2}$ and make it a normal insulator at some external pressure. 


\section{References}

[1] P. Hohenberg and W. Kohn, "Inhomogeneous electron gas," Phys. Rev., vol. 136, pp. B864-B871, 1964.

[2] W. Kohn and L. J. Sham, "Self-consistent equations including exchange and correlation effects," Phys. Rev., vol. 140, pp. A1133-A1138, 1965.

[3] J. P. Perdew and A. Zunger, "Self-interaction correction to density-functional approximations for many-electron systems," Phys. Rev. B, vol. 23, pp. 50485079, 1981.

[4] J. P. Perdew, J. A. Chevary, S. H. Vosko, K. A. Jackson, M. R. Pederson, D. J. Singh, and C. Fiolhais, "Atoms, molecules, solids, and surfaces - applications of the generalized gradient approximation for exchange and correlation," Phys. Rev. B, vol. 46, pp. 6671-6687, 1992.

[5] J. P. Perdew, J. A. Chevary, S. H. Vosko, K. A. Jackson, M. R. Pederson, D. J. Singh, and C. Fiolhais, "Erratum: Atoms, molecules, solids, and surfaces applications of the generalized gradient approximation for exchange and correlation," Phys. Rev. B, vol. 48, pp. 4978-4978, 1993.

[6] J. P. Perdew, K. Burke, and M. Ernzerhof, “Generalized gradient approximation made simple," Phys. Rev. Lett., vol. 77, pp. 3865-3868, 1996.

[7] D. R. Hamann, M. Schluter, and C. Chiang, “Norm-conserving pseudopotentials," Phys. Rev. Lett., vol. 43, p. 1494, 1979.

[8] P. E. Blöchl, "Projector augmented-wave method," Phys. Rev. B, vol. 50, pp. 17953-17979, 1994.

[9] G. Kresse and D. Joubert, "From ultrasoft pseudopotentials to the projector augmented-wave method," Phys. Rev. B, vol. 59, pp. 1758-1775, 1999.

[10] J. C. Slater and G. F. Koster, "Simplified LCAO method for the periodic potential problem," Phys. Rev., vol. 94, pp. 1498-1524, 1954.

[11] A. K. Geim and I. V. Grigorieva, "Van der Waals heterostructures," Nature, vol. 499, pp. 419-425, 2013.

[12] P. R. Wallace, “The band theory of graphite," Phys. Rev., vol. 71, pp. 622-634, 1947. 
[13] A. H. Castro Neto, F. Guinea, N. M. R. Peres, K. S. Novoselov, and A. K. Geim, "The electronic properties of graphene," Rev. Mod. Phys., vol. 81, pp. 109-162, 2009.

[14] A. Acun, L. Zhang, P. Bampoulis, M. Farmanbar, A. van Houselt, A. N. Rudenko, M. Lingenfelder, G. Brocks, B. Poelsema, M. I. Katsnelson, and H. J. W. Zandvliet, "Germanene: the germanium analogue of graphene," J. Phys.: Condens. Matter, vol. 27, p. 443002, 2015.

[15] K. S. Novoselov, A. K. Geim, S. V. Morozov, D. Jiang, Y. Zhang, S. V. Dubonos, I. V. Grigorieva, and A. A. Firsov, "Electric Field Effect in Atomically Thin Carbon Films," Science, vol. 306, pp. 666-669, 2004.

[16] L. Kou, Y. Ma, B. Yan, X. Tan, C. Chen, and S. C. Smith, "Encapsulated Silicene: A robust large-gap topological insulator," ACS Applied Materials $\mathcal{E}$ Interfaces, vol. 7, pp. 19226-19233, 2015.

[17] P. Tang, P. Chen, W. Cao, H. Huang, S. Cahangirov, L. Xian, Y. Xu, S.-C. Zhang, W. Duan, and A. Rubio, "Stable two-dimensional dumbbell stanene: A quantum spin Hall insulator," Phys. Rev. B, vol. 90, p. 121408, 2014.

[18] G. Giovannetti, P. A. Khomyakov, G. Brocks, P. J. Kelly, and J. van den Brink, "Substrate-induced band gap in graphene on hexagonal boron nitride: $\mathrm{Ab}$ initio density functional calculations," Phys. Rev. B, vol. 76, p. 073103, 2007.

[19] K. F. Mak, C. Lee, J. Hone, J. Shan, and T. F. Heinz, "Atomically Thin $\mathrm{MoS}_{2}$ : A New Direct-Gap Semiconductor," Phys. Rev. Lett., vol. 105, p. 136805, 2010.

[20] B. Radisavljevic, A. Radenovic, J. Brivio, V. Giacometti, and A. Kis, "SingleLayer MoS 2 Transistors," Nature Nanotechnology, vol. 6, pp. 147-150, 2011.

[21] M. S. Fuhrer and J. Hone, "Measurement of mobility in dual-gated $\operatorname{mos}_{2}$ transistors," Nature Nanotechnology, vol. 8, pp. 146-147, 2013.

[22] B. Radisavljevic and A. Kis, "Reply to "Measurement of mobility in dual-gated $\mathrm{MoS}_{2}$ transistors"," Nature Nanotechnology, vol. 8, pp. 147-148, 2013.

[23] G. W. Watson, S. C. Parker, and G. Kresse, "Ab initio calculations of the origin of the distortion of $\alpha-\mathrm{PbO}, "$ Phys. Rev. B, vol. 59, pp. 8481-8486, 1999.

[24] J. P. Allen, D. O. Scanlon, S. C. Parker, and G. W. Watson, "Tin Monoxide: Structural Prediction from First Principles Calculations with van der Waals Corrections," J. Phys. Chem. C, vol. 115, pp. 19916-19924, 2011.

[25] L. Fu, C. L. Kane, and E. J. Mele, "Topological insulators in three dimensions," Phys. Rev. Lett., vol. 98, p. 106803, 2007.

[26] M. Z. Hasan and C. L. Kane, "Colloquium: Topological insulators," Rev. Mod. Phys., vol. 82, pp. 3045-3067, 2010.

[27] M. V. Berry, "Quantal phase factors accompanying adiabatic changes," Proc. Roy. Soc. A, vol. 392, pp. 45-57, 1984. 
[28] D. J. Thouless, M. Kohmoto, M. P. Nightingale, and M. den Nijs, "Quantized Hall Conductance in a Two-Dimensional Periodic Potential," Phys. Rev. Lett., vol. 49, p. 405, 1982.

[29] C. L. Kane and E. J. Mele, " $Z_{2}$ Topological Order and the Quantum Spin Hall Effect," Phys. Rev. Lett., vol. 95, p. 146802, 2005.

[30] C. L. Kane and E. J. Mele, “Quantum Spin Hall Effect in Graphene,” Phys. Rev. Lett., vol. 95, p. 226801, 2005.

[31] T. Ando, T. Nakanishi, and R. Saito, "Berry's phase and absence of back scattering in carbon nanotubes," J. Phys. Soc. Jpn., vol. 67, pp. 2857-2862, 1998.

[32] L. Fu and C. L. Kane, "Topological insulators with inversion symmetry," Phys. Rev. B, vol. 76, p. 045302, 2007.

[33] R.-J. Slager, A. Mesaros, V. Juricic, and J. Zaanen, "The space group classification of topological band-insulators," Nature Physics, vol. 9, pp. 98-102, 2013.

[34] K. S. Novoselov, D. Jiang, F. Schedin, T. J. Booth, V. V. Khotkevich, S. V. Morozov, and A. K. Geim, "Two-dimensional atomic crystals," Proc. Natl. Acad. Sci. U.S.A., vol. 102, pp. 10451-10453, 2005.

[35] K. S. Novoselov, A. K. Geim, S. V. Morozov, D. Jiang, M. I. Katsnelson, I. V. Grigorieva, S. V. Dubonos, and A. A. Firsov, "Two-dimensional gas of massless Dirac fermions in graphene," Nature, vol. 438, pp. 197-200, 2005.

[36] Y. Zhang, Y.-W. Tan, H. L. Stormer, and P. Kim, “Experimental observation of the quantum Hall effect and Berry's phase in graphene," Nature, vol. 438, pp. 201-204, 2005.

[37] K. S. Novoselov, V. I. Fal'ko, L. Colombo, P. R. Gellert, M. G. Schwab, and K. Kim, “A roadmap for graphene," Nature, vol. 490, pp. 192-200, 2012.

[38] A. H. Castro Neto, F. Guinea, N. M. R. Peres, K. S. Novoselov, and A. K. Geim, "The electronic properties of graphene," Rev. Mod. Phys., vol. 81, pp. 109-162, 2009.

[39] F. Schwierz, "Graphene transistors: Status, prospects, and problems," Proceedings of the IEEE, vol. 101, pp. 1567-1584, 2013.

[40] J.-H. Chen, C. Jang, S. D. Xiao, M. Ishigami, and M. S. Fuhrer, "Intrinsic and extrinsic performance limits of graphene devices on $\mathrm{SiO}_{2}$," Nature Nanotechnology, vol. 3, pp. 206-209, 2008.

[41] S. Y. Zhou, G.-H. Gweon, A. V. Fedorov, P. N. First, W. A. De Heer, D.-H. Lee, F. Guinea, A. H. Castro Neto, and A. Lanzara, "Substrate-induced bandgap opening in epitaxial graphene," Nature Materials, vol. 6, pp. 770-775, 2007.

[42] C. R. Dean, A. F. Young, I. Meric, C. Lee, L. Wang, S. Sorgenfrei, K. Watanabe, T. Taniguchi, P. Kim, K. L. Shepard, and J. Hone, "Boron nitride substrates for high-quality graphene electronics," Nature Nanotechnology, vol. 5, pp. 722-726, 2010. 
[43] J. Xue, J. Sanchez-Yamagishi, D. Bulmash, P. Jacquod, A. Deshpande, K. Watanabe, T. Taniguchi, P. Jarillo-Herrero, and B. J. LeRoy, "Scanning tunnelling microscopy and specroscopy of ultra-flat graphene on hexagonal boron nitride," Nature Materials, vol. 10, pp. 282-284, 2011.

[44] R. Decker, Y. Wang, V. W. Brar, W. Regan, H. Tsai, Q. Wu, W. Gannett, A. Zettl, and M. F. Crommie, "Local Electronic Properties of Graphene on a BN Substrate via Scanning Tunneling Microscopy," Nano Letters, vol. 11, pp. 22912295, 2011.

[45] B. Sachs, T. O. Wehling, M. I. Katsnelson, and A. I. Lichtenstein, "Adhesion and electronic structure of graphene on hexagonal boron nitride substrates," Phys. Rev. B, vol. 84, p. 195414, 2011.

[46] L. A. Ponomarenko, A. K. Geim, A. A. Zhukov, R. Jalil, S. V. Morozov, K. S. Novoselov, I. V. Grigorieva, E. H. Hill, V. V. Cheianov, V. I. Fal'ko, K.Watanabe, T. Taniguchi, and R. V. Gorbachev, "Tunable metalinsulator transition in double-layer graphene heterostructures," Nature Physics, vol. 7, pp. 958-961, 2011.

[47] F. Amet, J. R. Williams, K.Watanabe, T.Taniguchi, and D. Goldhaber-Gordon, "Insulating behavior at the neutrality point in single-layer graphene," Phys. Rev. Lett., vol. 110, p. 216601, 2013.

[48] B. Hunt, J. D. Sanchez-Yamagishi, A. F. Young, K. Watanabe, T. Taniguchi, P. Moon, M. Koshino, P. Jarillo-Herrero, and R. C. Ashoori, "Massive Dirac fermions and Hofstadter butterfly in a van der Waals heterostructure," Science, vol. 340, pp. 1427-1430, 2013.

[49] F. Schwierz, "Graphene transistors," Nature Nanotechnology, vol. 5, pp. 487496, 2010.

[50] M. Kindermann, B. Uchoa, and D. L. Miller, "Zero-energy modes and gatetunable gap in graphene on hexagonal boron nitride," Phys. Rev. B, vol. 86, p. 115415, 2012.

[51] J. C. W. Song, A. V. Shytov, and L. S. Levitov, "Electron interactions and gap opening in graphene superlattices," Phys. Rev. Lett., vol. 111, p. 266801, 2013.

[52] M. Bokdam, T. Amlaki, G. Brocks, and P. J. Kelly, "Band gaps in incommensurable graphene on hexagonal boron nitride," Phys. Rev. B, vol. 89, p. 201404, 2014.

[53] P. San-Jose, A. Gutiérrez-Rubio, M. Sturla, and F. Guinea, "Spontaneous strains and gap in graphene on boron nitride," Phys. Rev. B, vol. 90, p. 075428, 2014.

[54] P. San-Jose, A. Gutiérrez-Rubio, M. Sturla, and F. Guinea, "Electronic structure of spontaneously strained graphene on hexagonal boron nitride," Phys. Rev. B, vol. 90, p. 115152, 2014. 
[55] J. Jung, A. M. DaSilva, A. H. MacDonald, and S. Adam, “Origin of band gaps in graphene on hexagonal boron nitride," Nature Communications, vol. 6, p. 6308, 2015.

[56] C. R. Woods, L. Britnell, A. Eckmann, R. S. Ma, J. C. Lu, H. M. Guo, X. Lin, G. L. Yu, Y. Cao, R. V. Gorbachev, A. V. Kretinin, J. Park, L. A. Ponomarenko, M. I. Katsnelson, Y. N. Gornostyrev, K. Watanabe, T. Taniguchi, C. Casiraghi, H.-J. Gao, A. K. Geim, and K. S. Novoselov, "Commensurate-incommensurate transition in graphene on hexagonal boron nitride," Nature Physics, vol. 10, pp. 451-456, 2014.

[57] M. Bokdam, G. Brocks, and P. J. Kelly, "Large potential steps at weakly interacting metal-insulator interfaces," Phys. Rev. B, vol. 90, p. 201411, 2014.

[58] G. Kresse and J. Hafner, "Ab-initio molecular-dynamics for liquid-metals," Phys. Rev. B, vol. 47, pp. 558-561, 1993.

[59] G. Kresse and J. Furthmüller, "Efficient iterative schemes for ab initio totalenergy calculations using a plane-wave basis set," Phys. Rev. B, vol. 54, pp. 11169-11186, 1996.

[60] M. Bokdam, P. A. Khomyakov, G. Brocks, Z. Zhong, and P. J. Kelly, "Electrostatic doping of graphene through ultrathin hexagonal boron nitride films," Nano Letters, vol. 11, pp. 4631-4635, 2011.

[61] M. Bokdam, P. A. Khomyakov, G. Brocks, and P. J. Kelly, "Field effect doping of graphene in metal|dielectric|graphene heterostructures: A model based upon first-principles calculations," Phys. Rev. B, vol. 87, p. 075414, 2013.

[62] R. O. Jones and O. Gunnarsson, "The density functional formalism, its applications and prospects," Rev. Mod. Phys., vol. 61, pp. 689-, 1989.

[63] G. Onida, L. Reining, and A. Rubio, "Electronic excitations: density-functional versus many-body green's-function approaches," Rev. Mod. Phys., vol. 74, pp. 601-659, 2002.

[64] L. Hedin, "New method for calculating the one-particle green's function with application to the electron-gas problem," Phys. Rev., vol. 139, pp. A796-A823, 1965.

[65] F. Hüser, T. Olsen, and K. S. Thygesen, "Quasiparticle gw calculations for solids, molecules, and two-dimensional materials," Phys. Rev. B, vol. 87, p. $235132,2013$.

[66] M. S. Hybertsen and S. G. Louie, "Electron correlation in semiconductors and insulators: Band gaps and quasiparticle energies," Phys. Rev. B, vol. 34, pp. 5390-5413, 1986.

[67] M. Shishkin and G. Kresse, "Implementation and performance of the frequency-dependent GW method within the PAW framework," Phys. Rev. B, vol. 74, p. 035101, 2006. 
[68] N. Berseneva, A. Gulans, A. V. Krasheninnikov, and R. M. Nieminen, “Electronic structure of boron nitride sheets doped with carbon from first-principles calculations," Phys. Rev. B, vol. 87, p. 035404, 2013.

[69] E. H. Hwang and S. Das Sarma, "Dielectric function, screening, and plasmons in two-dimensional graphene," Phys. Rev. B, vol. 75, p. 205418, 2007.

[70] G. J. Slotman, M. M. van Wijk, P.-L. Zhao, A. Fasolino, M. I. Katsnelson, and S. Yuan, "Effect of structural relaxation on the electronic structure of graphene on hexagonal boron nitride," Phys. Rev. Lett., vol. 115, p. 186801, 2015.

[71] S. Shallcross, S. Sharma, E. Kandelaki, and O. A. Pankratov, "Electronic structure of turbostratic graphene," Phys. Rev. B, vol. 81, p. 165105, 2010.

[72] J. Martin, N. Akerman, G. Ulbricht, T. Lohmann, J. H. Smet, K. von Klitzing, and A. Yacoby, "Observation of electron-hole puddles in graphene using a scanning single-electron transistor," Nature Physics, vol. 4, pp. 144-148, 2008.

[73] C.-H. Park, L. Yang, Y.-W. Son, M. L. Cohen, and S. G. Louie, "Anisotropic behaviours of massless dirac fermions in graphene under periodic potentials," Nature Physics, vol. 4, pp. 213-217, 2008.

[74] M. I. Katsnelson, "Nonlinear screening of charge impurities in graphene," Phys. Rev. B, vol. 74, p. 201401, 2006.

[75] P. A. Khomyakov, A. A. Starikov, G. Brocks, and P. J. Kelly, “Nonlinear screening of charges induced in graphene by metal contacts," Phys. Rev. B, vol. 82, p. $115437,2010$.

[76] T. Ando, "Screening effect and impurity scattering in monolayer graphene," J. Phys. Soc. Jpn., vol. 75, p. 074716, 2006.

[77] G.-H. Lee, Y.-J. Yu, C. Lee, C. Dean, K. L. Shepard, P. Kim, and J. Hone, “Electron tunneling through atomically flat and ultrathin hexagonal boron nitride," Appl. Phys. Lett., vol. 99, p. 243114, 2011.

[78] M. Dion, H. Rydberg, E. Schröder, D. C. Langreth, and B. I. Lundqvist, "Van der Waals Density Functional for General Geometries," Phys. Rev. Lett., vol. 92, p. 246401, 2004.

[79] G. Kresse and J. Furthmüller, "Efficiency of ab-initio total energy calculations for metals and semiconductors using a plane-wave basis set," Comp. Mat. Sci., vol. 6, pp. 15-50, 1996.

[80] P. E. Blöchl, O. Jepsen, and O. K. Andersen, "Improved tetrahedron method for Brillouin-zone integrations," Phys. Rev. B, vol. 49, pp. 16223-16233, 1994.

[81] J. Neugebauer and M. Scheffler, "Adsorbate-substrate and adsorbateadsorbate interactions of $\mathrm{Na}$ and K adlayers on Al(111)," Phys. Rev. B, vol. 46, pp. 16067-16080, 1992. 
[82] H. Rydberg, M. Dion, N. Jacobson, E. Schroder, P. Hyldgaard, S. I. Simak, D. C. Langreth, and B. I. Lundqvist, "Van der waals density functional for layered structures," Phys. Rev. Lett., vol. 91, p. 126402, 2003.

[83] K. I. Bolotin, F. Ghahari, M. D. Shulman, H. L. Stormer, and P. Kim, “Observation of the fractional quantum Hall effect in graphene," Nature, vol. 462, pp. 196-199, 2009.

[84] B. A. Bernevig, T. L. Hughes, and S.-C. Zhang, "Quantum spin Hall effect and topological phase transition in HgTe quantum wells," Science, vol. 314, pp. 1757-1761, 2006.

[85] M. König, S. Wiedmann, C. Brüne, A. Roth, H. Buhmann, L. W. Molenkamp, X.-L. Qi, and S.-C. Zhang, "Quantum Spin Hall Insulator State in HgTe Quantum Wells," Science, vol. 318, pp. 766-770, 2007.

[86] H. Min, J. E. Hill, N. A. Sinitsyn, B. R. Sahu, L. Kleinman, and A. H. MacDonald, "Intrinsic and Rashba spin-orbit interactions in graphene sheets," Phys. Rev. B, vol. 74, p. 165310, 2006.

[87] D. Huertas-Hernando, F. Guinea, and A. Brataas, "Spin-orbit coupling in curved graphene, fullerenes, nanotubes, and nanotube caps," Phys. Rev. B, vol. 74, p. 155426, 2006.

[88] Y. Yao, F. Ye, X.-L. Qi, S.-C. Zhang, and Z. Fang, "Spin-orbit gap of graphene: First-principles calculations," Phys. Rev. B, vol. 75, p. 041401, 2007.

[89] J. C. Boettger and S. B. Trickey, "First-principles calculation of the spin-orbit splitting in graphene," Phys. Rev. B, vol. 75, p. 121402, 2007.

[90] M. Gmitra, S. Konschuh, C. Ertler, C. Ambrosch-Draxl, and J. Fabian, “Bandstructure topologies of graphene: Spin-orbit coupling effects from first principles," Phys. Rev. B, vol. 80, p. 235431, 2009.

[91] A. H. Castro Neto and F. Guinea, "Impurity-Induced Spin-Orbit Coupling in Graphene," Phys. Rev. Lett., vol. 103, p. 026804, 2009.

[92] C. Weeks, J. Hu, J. Alicea, M. Franz, and R. Wu, “Engineering a robust quantum spin Hall state in graphene via adatom deposition," Phys. Rev. X, vol. 1, p. 021001, 2011.

[93] H. Zhang, C. Lazo, S. Blügel, S. Heinze, and Y. Mokrousov, "Electrically Tunable Quantum Anomalous Hall Effect in Graphene Decorated by $5 d$ TransitionMetal Adatoms," Phys. Rev. Lett., vol. 108, p. 056802, 2004.

[94] L. Kou, B. Yan, F. Hu, S.-C. Wu, T. O. Wehling, C. Felser, C. Chen, and T. Frauenheim, "Graphene-Based Topological Insulator with an Intrinsic Bulk Band Gap above Room Temperature," Nano Letters, vol. 13, pp. 6251-6255, 2013.

[95] T. P. Kaloni, L. Kou, T. Frauenheim, and U. Schwingenschlögl, "Quantum spin Hall states in graphene interacting with $\mathrm{WS}_{2}$ or WSe ${ }_{2}$," Appl. Phys. Lett., vol. 105, p. 233112, 2014. 
[96] L. Kou, F. Hu, B. Yan, T. O. Wehling, C. Felser, T. Frauenheim, and C. Chen, "Proximity enhanced quantum spin Hall state in graphene," Carbon, vol. 87, pp. 418-423, 2015.

[97] Z. Wang, D.-K. Ki, H. Chen, H. Berger, A. H. MacDonald, and A. F. Morpurgo, "Strong interface-induced spin-orbit interaction in graphene on $\mathrm{WS}_{2}$," Nature Communications, vol. 6, p. 8339, 2015.

[98] M. Gmitra and J. Fabian, "Graphene on transition-metal dichalcogenides: A platform for proximity spin-orbit physics and optospintronics," Phys. Rev. B, vol. 92, p. 155403, 2009.

[99] L. Kou, S.-C. Wu, C. Felser, T. Frauenheim, C. Chen, and B. Yan, "Robust 2D Topological Insulators in van der Waals Heterostructures," ACS Nano, vol. 8, pp. 10448-10454, 2014.

[100] Z. Y. Zhu, Y. C. Cheng, and U. Schwingenschlögl, “Giant spin-orbit-induced spin splitting in two-dimensional transition-metal dichalcogenide semiconductors," Phys. Rev. B, vol. 84, p. 153402, 2011.

[101] M. Yankowitz, J. Xue, and B. J. LeRoy, "Graphene on hexagonal boron nitride," J. Phys.: Condens. Matter, vol. 26, p. 303201, 2014.

[102] T. Thonhauser, V. R. Cooper, S. Li, A. Puzder, P. Hyldgaard, and D. C. Langreth, "Van der waals density functional: Self-consistent potential and the nature of the van der waals bond," Phys. Rev. B, vol. 76, p. 125112, 2007.

[103] J. Klimeš, D. R. Bowler, and A. Michaelides, "Van der waals density functionals applied to solids," Phys. Rev. B, vol. 83, p. 195131, 2011.

[104] G. Giovannetti, P. A. Khomyakov, G. Brocks, V. M. Karpan, J. van den Brink, and P. J. Kelly, "Doping graphene with metal contacts," Phys. Rev. Lett., vol. 101, p. 026803, 2008.

[105] P. A. Khomyakov, G. Giovannetti, P. C. Rusu, G. Brocks, J. van den Brink, and P. J. Kelly, "First-principles study of the interaction and charge transfer between graphene and metals," Phys. Rev. B, vol. 79, p. 195425, 2009.

[106] V. G. Ruiz, W. Liu, E. Zojer, M. Scheffler, and A. Tkatchenko, “DensityFunctional Theory with Screened van der Waals Interactions for the Modeling of Hybrid Inorganic-Organic Systems," Phys. Rev. Lett., vol. 108, p. 146103, 2012.

[107] T. Björkman, A. Gulans, A. V. Krasheninnikov, and R. M. Nieminen, "van der Waals Bonding in Layered Compounds from Advanced Density-Functional First-Principles Calculations," Phys. Rev. Lett., vol. 108, p. 235502, 2012.

[108] D. M. Ceperley and B. J. Alder, "Ground state of the electron gas by a stochastic method," Phys. Rev. Lett., vol. 45, pp. 566-569, 1980.

[109] F. Herman and S. Skillman, Atomic Structure Calculations. Englewood Cliffs, New Jersey: Prentice Hall, 1963. 
[110] Y. Ding, Y. Wang, J. Ni, L. Shi, S. Shi, and W. Tang, "First principles study of structural, vibrational and electronic properties of graphene-like $\mathrm{MX}_{2}(\mathrm{M}=$ Mo, Nb, W, Ta; X = S, Se, Te) monolayers," Physica B, vol. 406, pp. 2254-2260, 2011.

[111] C. Ataca, H. Şahin, and S. Ciraci, "Stable, Single-Layer $\mathrm{MX}_{2}$ Transition-Metal Oxides and Dichalcogenides in a Honeycomb-Like Structure," J. Phys. Chem. C, vol. 116, pp. 8983-8999, 2012.

[112] J. Kang, S. Tongay, J. Zhou, J. Li, and J. Wu, "Band offsets and heterostructures of two-dimensional semiconductors," Appl. Phys. Lett., vol. 102, p. 012111, 2013.

[113] E. Cappelluti, R. Roldán, A. Silva-Guillén, P. Ordejón, and F. Guinea, “Tightbinding model and direct-gap/indirect-gap transition in single-layer and multilayer $\operatorname{mos}_{2}$," Phys. Rev. B, vol. 88, p. 075409, 2013.

[114] L. Petersen and P. Hedegård, "A simple tight-binding model of spinorbit splitting of sp-derived surface states," Surface Science, vol. 459, pp. 49-56, 2000.

[115] B. Arnaud, S. Lebegue, P. Rabiller, and M. Alouani, "Huge excitonic effects in layered hexagonal boron nitride," Phys. Rev. Lett., vol. 96, p. 026402, 2006.

[116] Wolfram Research, Inc., SystemModeler. Champaign, Illinois: Wolfram Research, Inc., version 4.2 ed., 2015.

[117] A. M. Essin and J. E. Moore, "Topological insulators beyond the Brillouin zone via Chern parity," Phys. Rev. B, vol. 76, p. 165307, 2007.

[118] X.-L. Qi and S.-C. Zhang, "Topological insulators and superconductors," Rev. Mod. Phys., vol. 83, pp. 1057-1110, 2011.

[119] H. J. Terpstra, R. A. de Groot, and C. Haas, "Electronic structure of the lead monoxides: Band-structure calculations and photoelectron spectra," Phys. Rev. B, vol. 52, pp. 11690-11697, 1995.

[120] K. I. Bolotin, K. J. Sikes, Z. Jiang, M. Klima, G. Fudenberg, J. Hone, P. Kim, and H. L. Stormer, "Ultrahigh electron mobility in suspended graphene," Sol. State Comm., vol. 146, pp. 351-355, 2008.

[121] S. Balendhran, S. Walia, H. Nili, S. Sriram, and M. Bhaskaran, "Elemental analogues of Graphene: Silicene, Germanene, Stanene, and Phosphorene," Small, vol. 11, pp. 640-652, 2015.

[122] L. Zhang, P. Bampoulis, Q. Yao, B. Poelsema, and H. J. W. Zandvliet, "Synthesis and electronic decoupling of a 2D material: Germanene on $\mathrm{MoS}_{2}$," Adv. Mater., 2015.

[123] K. Takeda and K. Shiraishi, "Theoretical possibility of stage corrugation in Si and Ge analogs of graphite," Phys. Rev. B, vol. 50, pp. 14916-14922, 1994.

[124] G. G. Guzmán-Verri and L. C. Lew Yan Voon, "Electronic structure of siliconbased nanostructures," Phys. Rev. B, vol. 76, p. 075131, 2007. 
[125] S. Cahangirov, M. Topsakal, E. Aktürk, H. Şahin, and S. Ciraci, "Two- and onedimensional honeycomb structures of silicon and germanium," Phys. Rev. Lett., vol. 102, p. 236804, 2009.

[126] C.-C. Liu, W. Feng, and Y. Yao, "Quantum Spin Hall Effect in Silicene and twodimensional Germanium," Phys. Rev. Lett., vol. 107, p. 076802, 2011.

[127] L. C. Lew Yan Voon and G. G. Guzmán-Verri, “Is silicene the next graphene?,” Mater. Res. Soc. Bull., vol. 39, pp. 366-373, 2014. 


\section{Acknowledgement}

Even though that master equations in physics are simple, their outcome usually is very complicated. To me it seems that the same applies for our relations in life, where even simple interactions can have tremendous effects on a persons life. Hence I would like to use this opportunity to express my sincere gratitude toward people who had an important effect on me, and helped me to reach this point in my life.

First of all I would like to express my gratitude to my daily supervisor Prof. Dr. Paul J. Kelly for giving me the opportunity to pursue my PhD in his group. I learned from you that the key to scientific research is to always be critical of my own work. You give me lots of freedom in choosing my path and I think that have made me an independent researcher. I appreciate that your office was always open and you answered my questions patently, even at the time you had a very tight schedule. Your understanding of important physical properties of a system, enables you to suggest tests that were always very important in finding mistakes and incompleteness in my work and solve them, that is a scientific approach that I admire. Thank you for reviewing and editing my thesis, it took a lot of your time but you gave me helpful and detailed comments.

I would like to thank my phd committee, consisting of my promotor, Thomas Heine, Mikhail Katsnelson, Mark Golden, Allard Mosk, Harold Zandvliet, and Hans Hilgenkamp for taking the time to read this thesis and for making it possible for me to defend it in public.

My colleagues in the CMS group have given me great support all through my phd period. Geert, you always has an interesting topic about physics, history, language, and many other things. I enjoyed our discussions during my phd and for that I am thankful. Els, thank you for your secretary work and your kind help whenever I needed. Menno, collaborating with you has been very exciting and fruitful. Your first-principles calculations helped me to parametrize my tight binding model, and I hope that in our new project we can recreate the same experience. You helped me when I was learning VASP and assisted me in finding out how it works. I hope that you can continue your success in Vienna. Rien, thank you for helping me whenever I needed a Dutch speaking person and thanks for keeping our werkbespreking from being too long. I wish that you enjoy your new job. Zhe, thank you for teaching me about transport code, I hope you become even more successful in your career. Anton, you were always ready to answer me my questions, and you were passionate to discuss mathematical aspects of physical problems. I enjoyed our discussions a lot. Diana, Kriti, and Ireneus thank you for making my time in CMS so enjoyable.

Now, it is time to acknowledge my friends for sharing their moments with me. 
Mojtaba, we have known each other from Master. We worked together and applied for the same group for $\mathrm{PhD}$, and now we are both about to finish our PhD, maybe we will be colleague in future!. You have been a great friend for me, and always helped me in whatever problem I had, from filling forms to explaining to me how VASP works. You always helped me to see the positive side of life, where I have difficulty to see it ("Too koocheye ma ham aroosi mishe!"). Thank you for all of that and thank you for accepting to be my parafin!. I wish you the best in life. Hadi, Thank you for all your advices and guidance through my phd, and I don't forget the nights that we used to stay in university to finish our works. You showed me how the oscillation frequencies are measured in your lab and we discussed about how to make graphene by vibrating graphite. It didn't work but the experience was great. And thank you for accepting to be my paranimf. Thank you Damon for great vibe and bringing laughter to my life whenever I was down. I still remember our great times in "Disco Damon". Thank you for your help with making the cover of this thesis. Ali, you have a unique character that I admire. We have many memories from spending Christmas of 2012, which world did not end!, many discussions about physics, politics, and philosophy together. Your tough questions in physics, had helped me to improve my understanding in many cases. I wish you a better luck. Kasra, we have many talks about history that I enjoyed very much and for them I am thankful. I wish you luck in your new job. Milad and Mohammad, I had great times in your parties and appreciate your hospitality. I also like to thank Afshin, Omid, and Morteza, I had the best time with you.

It is time to acknowledge my family, my parents and my sister Maryam; thank you for your many years of supports and encouragement. Living away from my family has been difficult for me and I know that it has been difficult for them too. So, I want to thank them for their patience during these years. I also thank my in-law family for their understanding and helping me through my difficulties.

My deepest appreciation goes to my beloved wife and friend, Fahimeh. Your wisdom and kindness is a great source of inspiration and motivation in my life. Your patience in the last year of my $\mathrm{PhD}$ has made it possible for me to complete this thesis. I know that because I was behind from my schedule, my working hours has been irregular and long, but you never complained about it and always encouraged me to stay positive. I wish to be able to thank you for all of the sacrifices that you made, and I feel very lucky that I can have you beside me for my life.

Taher Amlaki

March 2016 

"An expert is a person who found out by his own painful experinece all the mistakes that one can make in a very narrow field."

Niels Bohr 